\title{
STRUCTURAL CHARACTERIZATION \\ AND BACTERIAL DEGRADATION \\ OF MARINE CARBOHYDRATES
}

by

Carol Arnosti

B.A., Lawrence University

(1984)

submitted in partial fulfillment of the requirements for the degree of

Doctor of Philosophy

BIOLOGICAL

LABORATORY

LIBRARY

WOOOS HOLE, MASS.

W. H. O. I.

\author{
at the \\ Massachusetts Institute of Technology \\ and the \\ and the \\ Woods Hole Oceanographic Institution \\ May 1993 \\ (c) Carol Arnosti 1993 \\ All rights reserved
}

The author hereby grants MIT and WHOI permission to reproduce and to distribute copies of this thesis document in whole or in part

Signature of Author

Joint Program in Oceanography,

Massachusetts Institute of Technology/Woods Hole Oceanographic Institution

Certified by
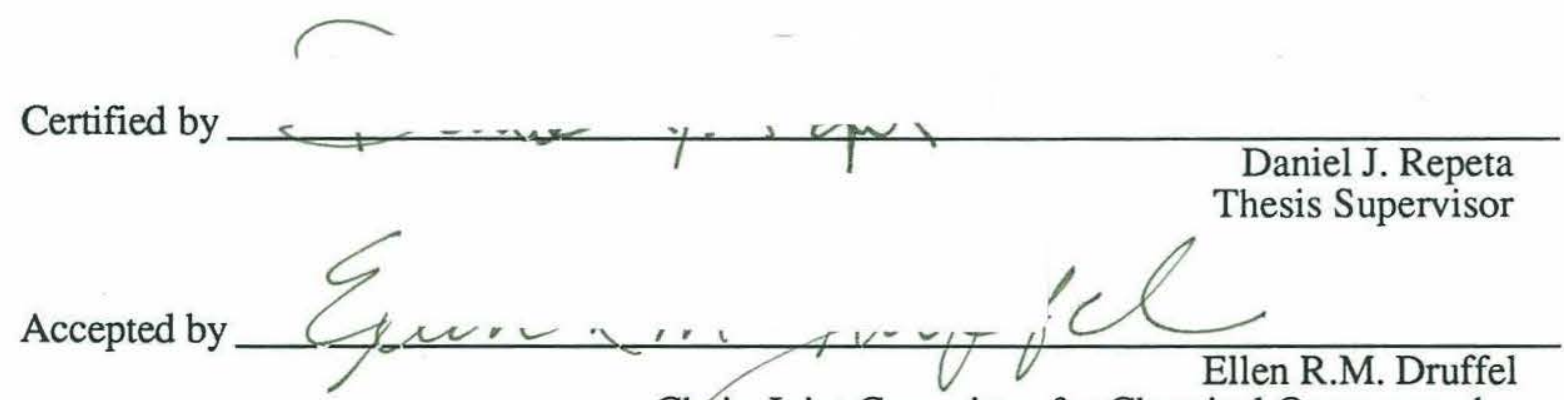

Chair, Joint Committee for Chemical Oceanography Massachusetts Institute of Technology/Woods Hole Oceanographic Institution

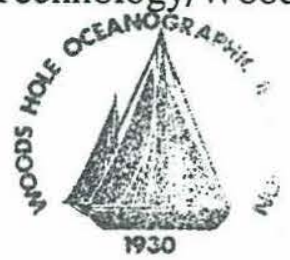




\title{
Structural Characterization and Bacterial Degradation of Marine Carbohydrates
}

\begin{abstract}
The goal of this thesis was to develop and apply an integrated chemical and microbial approach to study the effects of chemical structure on the rates and patterns of carbohydrate degradation by anaerobic marine bacteria.

Polysaccharides produced by five species of marine plankton, Dunaliella tertiolecta, Emiliania huxleyi, Stephanopixis palmeri, a Phaeocystis sp., and Synechococcus WH7335, were surveyed using one- and two-dimensional nuclear magnetic resonance spectroscopy (NMR). Two carbohydrates from Synechococcus WH7335 were characterized in detail. Synechococcus produced an $\alpha(1,4)$ glucose polysaccharide with $\alpha(1,6)$ branches, which probably functions as an energy reserve. The nominal molecular weight of the polysaccharide was $~ 5000$ daltons. Large quantities of a glycerolpolysaccharide, which was tentatively identified as a teichoic acid similar in structure to teichoic acids found in cell walls of gram-positive bacteria, were also produced by Synechococcus WH7335. This is the first report of teichoic acids in cyanobacteria.

Enrichment of bacteria from anoxic marine sediments on specific carbohydrates yielded reproducible model systems with which to study the degradation of chemically well-defined substrates. Headspace gases $\left(\mathrm{CO}_{2}, \mathrm{H}_{2}, \mathrm{CH}_{4}, \mathrm{H}_{2} \mathrm{~S}\right)$ were monitored by gas chromatography, and carbohydrate substrates and intermediates were separated and quantified via gel-permeation chromatography and high-pressure liquid chromatography. The transfer of carbon from substrates through to end products was followed quantitatively. Nuclear magnetic resonance spectroscopy was used to check for selective structural alterations (such as preferential cleavage of specific linkage types or positions) of the substrates.

A series of enrichment experiments showed that mixed cultures of marine bacteria distinguish even between small, very closely-related substrates which do not require extracellular hydrolysis prior to uptake. A galactose- $\beta(1,3)$-arabinose dimer was degraded at half the rate of seven other similar disaccharides and three larger oligosaccharides. A further series of degradation experiments with polysaccharides (pullulan, laminarin) showed that they are degraded by bacteria at virtually the same rate as structurally related substrates in the molecular weight range of 300-600 daltons. Degradation of the branched glucan and the teichoic acid-type polysaccharide from Synechococcus WH7335 was also very rapid.

The time-course of bacterial hydrolysis of pullulan was examined with gel permeation chromatography and NMR to provide the first molecular-level evidence in marine systems of the bacterial extracellular transformation of high molecular weight organic matter to lower molecular weight organic matter. NMR spectra provided evidence that the pullulan was hydrolyzed by pullulanase, an endo-acting extracellular enzyme which preferentially hydrolyzes $\alpha(1,6)$ linkages. This is the first experimental evidence of pullulanase activity among marine mesophilic bacteria.

The culture results suggest that enzymatic hydrolysis of macromolecular carbohydrates to transportable pieces is not the slow step in bacterial degradation of at least some types of polysaccharides. The results from the oligosaccharide experiments suggest that certain heteropolysaccharides may not be degraded as quickly. Chemical structure can be more important than molecular weight in determining degradation rates of carbohydrates. Varying rates of organic polymer degradation in anoxic sediments may be largely determined by the sensitivity of bacterial enzymatic and transport systems to structural features.
\end{abstract}


To my family

Now bid me run

and I will strive with things impossible

yea get the better of them

---Shakespeare

Julius Caesar, Act 2, Scene 1

The race is not always to the swift, but to those who keep on running ---runners' motto 


\section{Acknowledgements}

Many people contributed to the successful completion of this thesis ultramarathon; I am indebted to them all. My advisor, Dan Repeta, encouraged me to be independent and to follow the road less travelled. I have benefitted from his perspective and insight, and have come to admire his integrity and high scientific standards. Neil Blough, Craig Taylor, Phil Gschwend, and John Hedges made substantial contributions as members of my thesis committee, and I am grateful to them for discussions and advice. Carl Johnson and Bob Nelson contributed on a daily basis (in the form of nuts, bolts, reagents, glassware, tools, suggestions, and jokes) to my work; may the science gods smile on them both forever. I would also like to acknowledge Herman van Halbeek, of the Complex Carbohydrate Research Center at the University of Georgia, for his suggestions by fax and phone as I began the initial phases of the NMR work. A diverse collection of people in the biology department, including Freddie Valois, John Waterbury, Brian Howes, Dale Goehringer, Mark Dennet, and Joel Goldman generously provided help and equipment. I am particularly indebted to Freddie for introducing me to Synechococcus WH7335, and growing both of the cultures I used in this work.

Special thanks go to Kathleen Ruttenberg, Sarah Green, Ralf Goericke, and Susie McGroddy for sharing the good times and the bad, as well as their coffee, honey, chocolate, and crackers (respectively) and their advice and perspectives (collectively), all of which were greatly appreciated. Thanks are also due to many other Fye Folks, especially Tim and Lorraine Eglinton, Jean Whelan, Dan Simpson, Lary Ball, Sigi Caron, Mary Zawoyski, and Carla Bold. My fellow WHOI divers, Ellyn Montgomery and Tom Bolmer, Martha Tarafa, Maggie and Terry Rioux, and Alan Fleer, helped provide the underwater perspective on many a sunny summer afternoon. John Kokinos, Alan Kuo, Cheri Recchia, Kirby Olson, Jon Caulkins, Carolyn Lee and Julian Betts, Birgit Angern-Dorgarten and Hans-Wilhelm Dorgarten, and Adi Blob and Maria Ney-Blob have shared the ups and downs of graduate school with me, and helped me maintain my sense of humor throughout. Elise Ralph, Chris Bradley, Laura Praderio, and Sarah Green, my housemates and friends, put up with my odd hours, helped me host many memorable "feeding frenzies," and welcomed my international collection of visitors.

This thesis is dedicated to my family: to my brothers and sister, who got me off to an early start in science and sports, and who have continued, with their families, to be my boosters and friends. Most especially, it is dedicated to my parents, whom I want to thank for their unwavering support in all of my endeavors, and for instilling a love of learning and a spirit of discovery in all of us. 
Funding for this work was provided by a graduate fellowship from the Office of Naval Research, and grants from the WHOI Ocean Ventures Fund and the Department of Energy's Ocean Margins Program (DE-FG02-92ER61428). 


\section{Table of Contents}

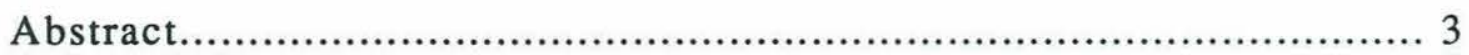

Acknowledgements...................................................................... 5

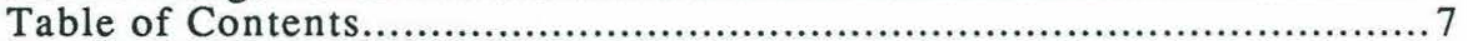

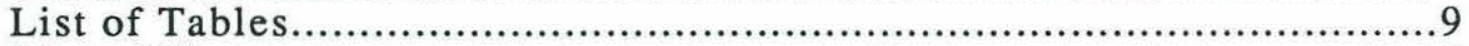

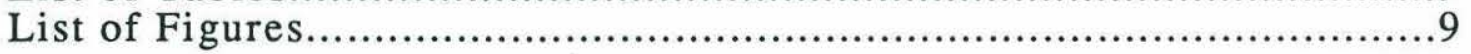

Chapter 1: Introduction

Overview.................................................... 13

Degradation of Organic Matter.................................. 15

Bacteria in Anoxic Sediments ................................... 17

Bacterial Consortia..................................... 18

Enzymes and Porins................................. 19

Previous Studies of Early Diagenesis........................... 20

Marine Carbohydrates........................................... 22

Terminology and General Structures.................... 22

Analysis of Marine Carbohydrates..................... 22

Carbohydrates of Marine Organisms............ 26

Dissolved Carbohydrates......................... 26

Particulate Carbohydrates........................ 28

Carbohydrates in Sediments..................... 29

Summary: Implications for the Degradation of Marine Carbohydrates............... 30

Thesis Goal: A New Approach to Studying Early Diagenesis 32 References..................................................... 34

Chapter 2: $\quad$ Nuclear Magnetic Resonance Spectroscopy of Carbohydrates

Introduction............................................... 41

Experimental Conditions..................................... 42

One- and Two-Dimensional NMR............................. 43

Application of NMR to Studies of Carbohydrates............. 47

Survey of Carbohydrate Standards............................. 51

Oligosaccharides..................................... 52

1D Spectra.................................... 52

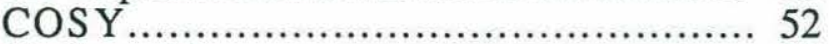

Carbon-13................................... 55

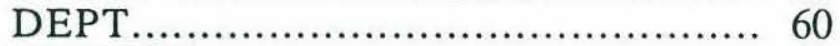

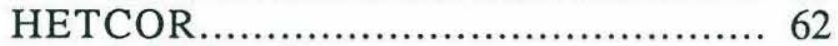

Maltose and Maltotriose.................................. 66

Polysaccharides................................... 66

Amylose...................................... 69

Laminarin.................................... 69

Pullulan.......................................... 69

References....................................... 79

Chapter 3: $\quad$ Plankton Carbohydrates

Introduction............................................. 83

Phytoplankton Carbohydrates............................... 84

Survey of Phytoplankton Carbohydrates........................ 85

Overview of Organisms................................. 85 
Cultures........................................ 86

Excreted Carbohydrates............................. 87

Extraction of Cellular Carbohydrates..................... 87

Hot Aqueous Extracts........................... 88

Cold Aqueous Extracts....................... 95

The Carbohydrates of Synechococcus WH7335............. 98

The Carbohydrates of Cyanobacteria................. 98

Extraction of Synechococcus WH7335................ 100

Fractionation of the Cold Aqueous Extract............. 100

NMR Analysis of Fractions 1-5....................... 104

Fraction 2........................................ 104

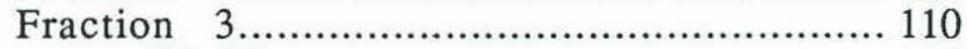

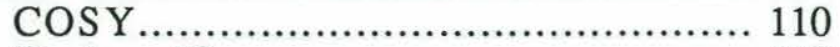

Carbon-13................................... 110

DEPT ...................................... 115

HETCOR ................................... 117

Compositional and Linkage Analysis.......... 122

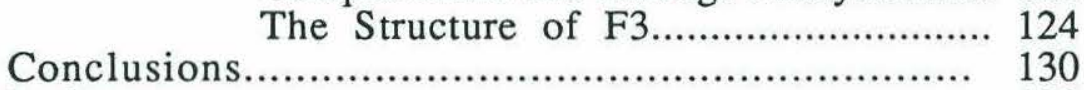

References............................................ 132

Chapter 4: Effect of Chemical Structure on Carbohydrate Degradation

by Anaerobic Marine Bacteria: Degradation of Di- and Oligosaccharides

Introduction............................................ 137

Methods................................................. 139

Substrates...................................... 139

Preparation of Substrates and Inoculum................ 143

Culture Sampling..................................... 143

Headspace Gas Measurement........................... 143

Substrate Quantification............................... 144

Total $\mathrm{CO}_{2}$ Determination............................. 146

Blanks............................................ 146

Results and Discussion..................................... 147

General Results................................ 147

Reproducibility .................................. 150

Homo- and Heterodisaccharides...................... 151

Degradation of GLA................................ 159

Degradation of Maltooligosaccharides.................. 162

Conclusions................................................ 164

References................................................ 166

Chapter 5: Effect of Chemical Structure on Carbohydrate Degradation

by Anaerobic Marine Bacteria: Degradation of Polysaccharides

Introduction................................................... 169

Methods............................................... 171

Substrates................................... 171

Culture methods.................................... 173

Culture Sampling ................................. 173

Sample Analysis................................... 173

Results and Discussion....................................... 175

Reproducibility and General Results................... 175

Substrate Degradation................................. 179

Detailed Analysis of Pullulan Degradation............. 186

Enzyme Specificity..................................... 202 


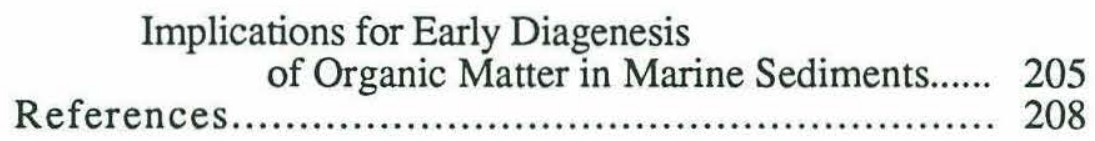

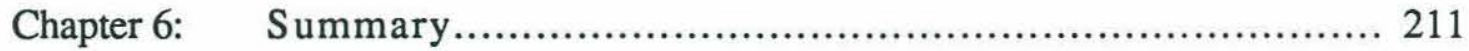

Appendix A: Acquisition and Processing Parameters

for ${ }^{13} \mathrm{C}$ and 2D NMR Spectra............................... 219

Appendix B: Survey of Phytoplankton Carbohydrates.............................. 224

\section{List of Tables}

Chapter 2

Table 2.1: Composition of reducing sugars in solution............................. 49

Table 2.2: $\quad{ }^{1} \mathrm{H}$ chemical shifts of gentiobiose.................................... 56

Table 2.3: $\quad{ }^{13} \mathrm{C}$ chemical shifts of isomaltose...................................... 61

Table 2.4: $\quad{ }^{1} \mathrm{H}$ chemical shifts of isomaltose....................................... 65

Chapter 3

Table 3.1: $\quad$ Extract yields and CHN results for five species of plankton.......... 89

Table 3.2: $\quad$ Monosaccaride composition and linkage analysis of $\mathrm{F} 3$.............. 123

Table 3.3: $\quad{ }^{13} \mathrm{C}$ chemical shifts for F3 from Synechococcus WH7335.......... 129

Chapter 4

Table 4.1: Artificial seawater composition 140

\section{List of Figures}

Chapter 1

Fig. 1.1:

Fig. 1.2:

Fig. 1.3:

Fig. 1.4:

Chapter 2

Fig. 2.1:

Fig. 2.2:

Fig. 2.3:

Fig. 2.4:

Fig. 2.5:

Fig. 2.6:

Fig. 2.7:

Fig. 2.8:

Fig. 2.9:

Fig. 2.10:

Fig. 2.11:
Schematic model of organic transformation in sediments.............. 16

Basic carbohydrate structures.......................................... 23

The eight most common neutral monosaccharides ..................... 23

Disaccharide, oligosaccharide, and polysaccharide structures......... 24

Spin-1/2 nuclei in an external magnetic field............................ 44

Effect of a radiofrequency pulse on the net magnetization vector...... 44

Vector-model of a two-dimensional NMR experiment.................... 46

${ }^{1} \mathrm{H}$ spectrum of $\beta$-glucose in solution...................................... 48

${ }^{1} \mathrm{H}$ spectrum of an equilibrium mixture of $\alpha$ - and $\beta$-glucose

in solution.............. 50

${ }^{1} \mathrm{H}$ spectra of isomaltose and gentiobiose................................. 53

Gentiobiose ${ }^{1} \mathrm{H}$ COSY $45^{\circ}$. ........................................ 54

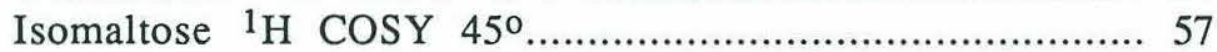

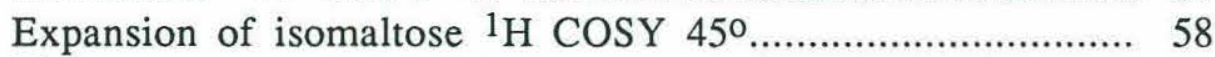

${ }^{13} \mathrm{C}$ spectrum of isomaltose.............................................. 59

DEPT experiments for isomaltose: (a) DEPT $135^{\circ}$, (b) DEPT $90^{\circ}$,

(c) DEPT $45^{\circ}$ 
Fig. 2.12: $\quad{ }^{1} \mathrm{H}-13 \mathrm{C}$ correlation experiment (HETCOR) for isomaltose............ 64

Fig. 2.13: $\quad{ }^{1} \mathrm{H}$ COSY $45^{\circ}$ of maltose.............................................. 67

Fig. 2.14: $\quad{ }^{1} \mathrm{H}$ COSY $45^{\circ}$ of maltotriose............................................ 68

Fig. 2.15: $\quad{ }^{1} \mathrm{H}$ spectrum of amylose........................................... 70

Fig. 2.16: $\quad{ }^{13} \mathrm{C}$ spectrum of amylose................................................ 71

Fig. 2.17: $\quad{ }^{1} \mathrm{H}$ spectrum of laminarin............................................... 72

Fig. 2.18: $\quad{ }^{1} \mathrm{H}$ COSY of pullulan................................................... 73

Fig. 2.19: $\quad{ }^{13} \mathrm{C}$ spectrum of pullulan................................................... 74

Fig. 2.20: $\quad{ }^{1} \mathrm{H}-{ }^{13} \mathrm{C}$ correlation experiment (HETCOR) of pullulan................. 77

Fig. 2.21: Expansion of pullulan HETCOR.......................................... 78

Chapter 3

Fig. 3.1a-c: Anomeric regions of Stephanopixis, Phaeocystis, and Phaeocystis hydrolyzed hot aqueous extracts..................................... 90

Fig. 3.1d-f: Anomeric regions of E. huxleyi, Synechococcus WH7335, and Synechococcus WH7335 hydrolyzed hot aqueous extracts.. 91

Fig. 3.2: $\quad{ }^{1} \mathrm{H}$ NMR spectrum of hot aqueous extract of Dunaliella tertiolecta... 93

Fig. 3.3: $\quad$ Partial ${ }^{1} \mathrm{H}$ NMR spectrum of Dunaliella tertiolecta hot aqueous extract and ${ }^{1} \mathrm{H}$ NMR spectrum of amylose standard........................... 94

Fig. 3.4a-d: Anomeric regions of Dunaliella, Stephanopixis, Phaeocystis, and E. huxleyi cold aqueous extracts.............................. 96

Fig. 3.5: $\quad{ }^{1}$ H NMR spectrum ofSynechococcus WH7335 cold aqueous extract............................................ 101

Fig. 3.6: Gel permeation chromatography chromatogram of Synechococcus WH7335 cold aqueous extract................ 103

Fig. 3.7: $\quad{ }^{1} \mathrm{H}$ NMR spectrum of F4 (54-64 minutes, GPC column) from Synechococcus WH7335 cold aqueous extract......... 105

Fig. 3.8: $\quad{ }^{1} \mathrm{H}$ NMR spectrum of F2 (22-31 minutes, GPC column) from Synechococcus WH7335 cold aqueous extract......... 106

Fig. 3.9: $\quad$ Anomeric region of ${ }^{1} \mathrm{H}$ COSY of $\mathrm{F} 2$ from Synechococcus WH7335............................... 107

Fig. 3.10: $\quad$ Proposed structure of F2 from Synechococcus WH7335 cold aqueous extract............................................ 109

Fig. 3.11: $\quad{ }^{1} \mathrm{H}$ NMR spectrum of F3 (31-48 minutes, GPC column) from Synechococcus WH7335 cold aqueous extract......... 111

Fig. 3.12: $\quad{ }^{1} \mathrm{H}$ COSY of F3 from Synechococcus WH7335 cold aqueous extract........................................ 112

Fig. 3.13: Expansion of the anomeric region of the ${ }^{1} \mathrm{H}$ COSY of F3........... 113

Fig. 3.14: $\quad{ }^{13} \mathrm{C}$ spectrum of F3 from Synechococcus WH7335 cold aqueous extract......................................... 114

Fig. 3.15: DEPT spectra of F3 .......................................... 116

Fig. 3.16: $\quad{ }^{1} \mathrm{H}-{ }^{13} \mathrm{C}$ HETCOR of $\mathrm{F} 3$ from Synechococcus WH7335 cold aqueous extract.

Fig. 3.17: $\quad{ }^{1} \mathrm{H}$ COSY of F3 from Synechococcus WH7335 cold aqueous extract........................................... 119

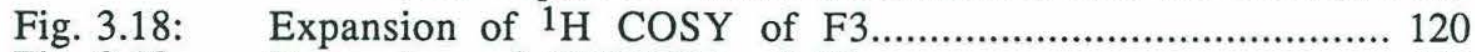

Fig. 3.19: Expansion of HETCOR of F3

Fig. 3.20: Proposed structure of F3 from Synechococcus WH7335 cold aqueous extract............................................ 125 
Chapter 4

Fig. 4.1:

Fig. 4.2:

Fig. 4.3:

Fig. 4.4:

Fig. 4.5:

Fig. 4.6:

Fig. 4.7:

Fig. 4.8:

Fig. 4.9:

Fig. 4.10:

Fig. 4.11:

Fig. 4.12:

Chapter 5

Fig. 5.1:

Fig. 5.2:

Fig. 5.3:

Fig. 5.4:

Fig. 5.5:

Fig. 5.6:

Fig. 5.7:

Fig. 5.8:

Fig. 5.9:

Fig. 5.10:

Fig. 5.11:

Fig. 5.12:

Fig. 5.13:

Fig. 5.14:

Fig. 5.15:

Fig. 5.16:

Fig. 5.17:

Fig. 5.18:
Flow diagram of culture experimental procedures 141

Structures of di- and oligosaccharide substrates..................... 142

Separation of DP1-6 maltooligosaccharides using HPLC............ 145

Degradation of maltose and evolution of headspace gases........... 148

Headspace $\mathrm{CO}_{2}$ profiles for maltose................................ 152

Headspace $\mathrm{CO}_{2}$ profiles for lactose................................. 153

Headspace $\mathrm{CO}_{2}$ profiles for disaccharide substrates................. 154

Substrate degradation profiles for disaccharide substrates........... 156

Degradation of maltose and GLA, and evolution of total $\mathrm{CO}_{2} \ldots \ldots . .157$

$\mathrm{pH}$ and headspace $\mathrm{CO}_{2}$ for maltose and GLA cultures............... 158

Headspace $\mathrm{CO}_{2}$ for GLA culture and transfer culture................. 160

Substrate degradation and evolution of total $\mathrm{CO}_{2}$

for maltooligosaccharide series.

162

Production and consumption of headspace $\mathrm{H}_{2}$ for all cultures........ 177

Production of headspace $\mathrm{CO}_{2}$ and recovery of carbon as $\mathrm{CO}_{2} \ldots \ldots . .178$

Time-course of F2, F3, pullulan, and laminarin degradation......... 180

${ }^{1} \mathrm{H}$ NMR spectrum of anomeric region of $\mathrm{F} 2$ at zero-time and 69 hours

181

${ }^{1} \mathrm{H}$ NMR spectrum of anomeric region of F3 at zero-time, 8 hours, 16 hours, and 25.5 hours

${ }^{1} \mathrm{H}$ NMR spectrum of anomeric region of laminarin at zero-time, 8 hours, 16 hours, 25.5 hours, 30 hours, and 36 hours

184

G50 gel permeation chromatogram of pullulan (zero hours)......... 187

G50 gel permeation chromatogram of pullulan (49.5 hours)........ 188

G50 gel permeation chromatogram of pullulan (56 hours)........... 189

G50 gel permeation chromatogram of pullulan (60 hours).......... 190

G50 gel permeation chromatogram of pullulan (64 hours)........... 191

${ }^{1} \mathrm{H}$ NMR spectrum of three gel permeation chromatography fractions of pullulan (zero time): $\geq 10,000$ daltons (a), $\sim 5000$ daltons (b), $\leq 1200$ daltons (c).

${ }^{1} \mathrm{H}$ NMR spectrum of three gel permeation chromatography fractions of pullulan (40 hours): $\geq 10,000$ daltons (a), $\sim 5000$ daltons (b), $\leq 1200$ daltons (c)

${ }^{1} \mathrm{H}$ NMR spectrum of three gel permeation chromatography fractions of pullulan (49.5 hours): $\geq 10,000$ daltons (a), $\sim 5000$ daltons (b), $\leq 1200$ daltons (c).

${ }^{1} \mathrm{H}$ NMR spectrum of three gel permeation chromatography fractions of pullulan (56 hours): $\geq 10,000$ daltons (a), $\sim 5000$ daltons (b), $\leq 1200$ daltons (c).

${ }^{1} \mathrm{H}$ NMR spectrum of three gel permeation chromatography fractions of pullulan ( 60 hours): $\geq 10,000$ daltons (a), $\sim 5000$ daltons (b), $\leq 1200$ daltons (c)

${ }^{1} \mathrm{H}$ NMR spectrum of three gel permeation chromatography fractions of pullulan (64 hours): $\geq 10,000$ daltons (a), $\sim 5000$ daltons (b), $\leq 1200$ daltons (c) fractions of pullulan ( 69 hours): $\geq 10,000$ daltons (a), 
$\sim 5000$ daltons (b), $\leq 1200$ daltons (c).................... 199

Fig. 5.19: Summary of changes in pullulan size classes during degradation.... 200

Fig. 5.20: Bar chart of changes in distribution of substrate among

$\geq 10,000$ dalton, $\sim 5000$ dalton, and $\leq 1200$ dalton size classes

during pullulan degradation................................. 201

Fig. 5.21: High-resolution ${ }^{1} \mathrm{H}$ NMR spectrum of $\leq 1200$ dalton

gel permeation fraction of pullulan (60 hours).

(Same fraction as Fig. 5.16c)............................... 203 


\section{Chapter One: Introduction}

\section{Overview}

Phytoplankton in the surface ocean use $\mathrm{CO}_{2}$ fixed via photosynthesis to synthesize macromolecular organic compounds such as proteins, lipid complexes, and polysaccharides. The majority of this macromolecular organic matter is remineralized back to $\mathrm{CO}_{2}$ either in the surface ocean or in the sediments (Henrichs, 1992). The factors controlling the remineralization of organic macromolecules are not well understood, however; our limited ability to structurally characterize intact macromolecules is a major barrier to determining rates and mechanisms of organic matter degradation in marine systems. Although most organic matter in living organisms is macromolecular, research in organic geochemistry has focused on lower molecular weight components or on hydrolysis products (amino acids, monosaccharides) of the high molecular weight material. An important aspect of marine organic matter, therefore, is largely unknown. Because we know little about the form in which organic matter is produced, we do not know which factors control the rates and processes by which organic matter is cycled in the water column and sediments, or why nominally labile components of organic matter (carbohydrates and proteins, measured as monosaccharides (Cowie and Hedges, 1984) and amino acids (Henrichs, 1992)) apparently are resistent to degradation beyond a given depth in recent sediments.

A major proportion of macromolecular organic matter consists of carbohydrates, high molecular-weight polymers of simple sugars. In phytoplankton, for example, carbohydrates comprise $20-40 \%$ of cellular biomass (Parsons et al., 1961). Carbohydrates are used as energy reserves and for structural support (Lee, 1980), and are integral components of membrane and cellular signalling systems (Bishop and Jennings, 1982).

There is a wide range in the relative lability of different carbohydrates in marine environments. Glucose (a simple sugar) fuels the basic metabolic pathways of most organisms and is quickly degraded in seawater and sediments (Vaccaro and Jannasch, 1966; Takahashi and Ichimura, 1971; Meyer-Reil, 1978; Gocke et al., 1981). The presence of carbohydrates (including glucose) in ancient sediments (Whelan and Emeis, 1992), however, shows that some portion of carbohydrates resist degradation and are preserved in sediments. This range of degradability exists even though individual monosaccharides are structurally quite homogeneous. High molecular weight carbohydrates are classic biological polymers, differing in the number of repeating units, varying somewhat in monomer composition, and having a well-defined range of linking and cross-linking possibilities. We 
do not know which aspects of structure influence the extent of carbohydrate decomposition. Cellulose, for example, is a linear polymer of glucose, yet most organisms are unable to degrade cellulose, even though its component monosaccharide is easily degraded.

Bacteria are ultimately responsible for metabolizing and recycling much of the organic carbon fixed by marine phytoplankton (Fuhrman and Azam, 1982; Azam et al., 1983). In anoxic sediments, bacteria are the only organisms capable of degrading complex organic matter to simpler substances and $\mathrm{CO}_{2}$. While the bacterial degradation of substances such as fatty acids and simple sugars is well understood (Gottschalk, 1986), little is known about the pathways by which bacteria degrade the macromolecular material which comprises most of the organic matter in the environment. The goal of this thesis is to investigate the bacterial degradation of complex carbohydrates in marine systems, focusing on the effects of the chemical structure on degradation rates and patterns. Structure is a critical parameter because there is a finite limit of approximately 600 daltons on the molecular weight of substrate which a bacterium can ingest (Weiss et al., 1991). Above this exclusion limit, organic matter must be degraded to smaller pieces outside of the bacterial cell by exoenzymes that are selective for specific linkage types and positions (Priest, 1992).

Carbohydrates were selected as a focal point of this work because they are compositionally well-suited to serve as model compounds in a study of structural effects on degradation rates and patterns. Unlike proteins and lipids, carbohydrate macromolecules usually vary in a structurally well-defined manner. Proteins are made of combinations of twenty amino acids linked by a peptide bond. Polysaccharides are typically made of only a few closely-related monomers, with a vast array of linkage possibilities. In addition, although carbohydrates comprise a high percentage of marine organic carbon, as a class they have not been studied as thoroughly as lipids and amino acids in marine environments.

The next section of this chapter briefly reviews organic matter degradation, bacterial communities in anoxic sediments, and geochemical studies of early diagenesis. The following section includes an overview of carbohydrate terminology and structure, and a brief survey of previous work on the sources and fates of marine carbohydrates. The chapter concludes with an outline of the general approach and specific goals of the thesis.

Chapter 2 includes a basic introduction to one- and two-dimensional nuclear magnetic resonance spectroscopy (NMR) of carbohydrates, and results of a survey of carbohydrate standards. Nuclear magnetic resonance spectroscopy is used to characterize phytoplankton extracts in Chapter 3, and the cellular carbohydrates of a marine cyanobacterium are characterized in detail. Chapters 4 and 5 present results of a series of 
experiments on the effects of chemical structure on oligo- and polysaccharide degradation by anaerobic marine bacteria. Chapter 6 summarizes the work, and includes some suggestions for further work on characterization and bacterial degradation of marine carbohydrates.

\section{Degradation of Organic Matter}

The sediment-water interface is a site of active remineralization of organic matter by benthic organisms; very little of the organic matter reaching the sediment-water interface is ultimately buried in the sediments (Emerson and Hedges, 1988; Hedges, 1992). Some aspects of organic matter transformations within the sediments are reasonably well understood, as illustrated in Fig. 1.1. The flux of organic carbon to the sediment-water interface (A), and burial of organic carbon within the sediments (B), can be constrained using sediment traps and by analysis of the organic carbon content of sediments, respectively. The flux of methane, ammonia, and total $\mathrm{CO}_{2}$ out of the sediments (I) has been measured with benthic flux chambers, while pore water analyses have provided information on the burial of total $\mathrm{CO}_{2}$, dissolved monomers, and (to some extent) dissolved polymers (J, K, L, respectively). The transformation of dissolved monomers to total $\mathrm{CO}_{2}$, ammonia, and methane $(\mathrm{F})$ has been measured both in sediment systems and in pure cultures of bacteria. In contrast, the rates and processes of transformations between the three major carbon reservoirs within the sediments (particulate macromolecules $\rightarrow$ dissolved macromolecules $\longrightarrow$ dissolved monomers, (C, D, and E)) are relatively unknown.

The transformation and degradation of macromolecules within the sediments may be influenced by a variety of factors. Preservation of organic carbon has been explicitly linked with slow degradation rates. Degradation rate may be slowed by the specific chemical structure of organic matter. Studies of bacterial degradation of specific substrates clearly demonstrate that chemical structure can affect relative degradation rates. Free amino acids, for example, are swiftly degraded by anaerobic bacteria, while saturated hydrocarbons are resistant to degradation (Schink, 1989).

Extrapolation of pure culture studies to the degradation of macromolecules in sediments is difficult, however, since these studies are not intended to investigate degradation of high molecular weight organic matter. The low molecular weight structures used as substrates (often simple aromatic or halogenated compounds) are not applicable models for natural macromolecular structures (Weimer and Zeikus, 1977; Bernier and Stutzenberger, 1987; Hespell et al., 1987; van den Tweel et al., 1987; Heitkamp et al., 


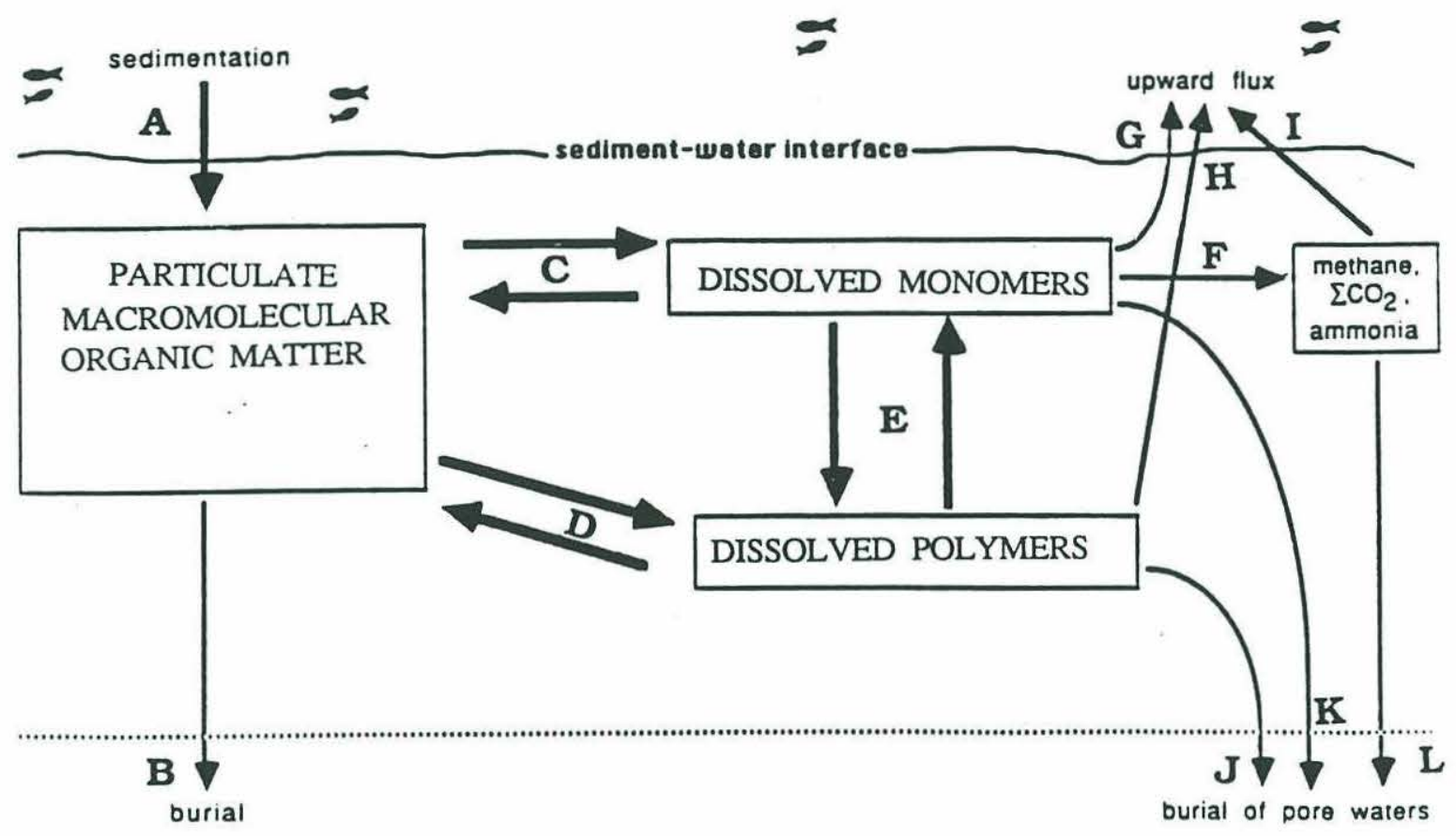

(adapted from Burdige and Martens, 1988)

Fig. 1.1

Model of organic matter transformations in the sediments (adapted from Burdige and Martens, 1988). Arrows depict transfer of organic carbon. Sedimentation of organic matter (A), burial of organic matter (B), transfer between particulate macromolecular organic matter and dissolved monomer boxes (C), transfer between dissolved polymers and particulate macromolecular organic matter boxes (D), transfer between dissolved polymer and dissolved monomer boxes (D), transfer of dissolved monomers to methane, $\mathrm{CO}_{2}$, and ammonia (F), upward flux from dissolved monomer box (G), upward flux from dissolved polymer box $(\mathrm{H})$, upward flux from methane, total $\mathrm{CO}_{2}$, ammonia box (I), burial of dissolved polymers in porewater $(\mathrm{J})$, burial of dissolved monomers in porewater $(\mathrm{K})$, burial of methane/total $\mathrm{CO}_{2} /$ ammonia in porewaters $(\mathrm{L})$. 
1988). In addition, pure or co-cultures of bacteria do not adequately represent the metabolic versatility of microbial communities in anoxic sediments. Pure culture studies of microbial transformations also suffer from the inherent limitation that microbes which can be grown in culture represent perhaps $1 \%$ of the total population (Fuhrman et al., 1992), and the metabolic capabilities of most of the bacterial population therefore are unknown.

Packaging, particle interaction, and humification reactions all may hinder the degradation of nominally labile organic matter to the extent that it is ultimately buried in the sediments. Harvey et al. (1986) found that the degradation of bacterial lipids in sediments of higher organic carbon content was consistently lower than degradation in sediments with low organic carbon content, under both oxic and anoxic conditions. They suggested that sorption of lipid to organic matter could reduce its availability to microorganisms. Packaging may account for preservation of labile organic matter components, such as protection of pectin by lignin (Emerson and Hedges, 1988). Specific mechanisms which may be responsible for preservation of otherwise labile organic matter may occur through transformation into "geomacromolecules" (Tissot and Welte, 1984), humification reactions, or incorporation of inorganic sulfur, as discussed in a recent review (Henrichs, 1992).

\section{Bacteria in Anoxic Sediments}

The fact that anoxic sediments account for more than $90 \%$ of the annual burial of marine organic carbon (Henrichs and Reeburgh, 1987), has led to the suggestion that lack of oxygen slows the degradation of organic matter, and by extension enhances organic matter preservation (Emerson, 1985, for example). Laboratory incubation studies have shown rate differences between oxic and anoxic degradation of bacterial membrane lipids (Harvey et al., 1986). The degradation of specific organic matter classes such as lignin undoubtedly proceeds more slowly in the absence of oxygen (Benner et al., 1984a; Benner et al., 1984b), but a wide variety of studies has shown that degradation rates of organic matter under anoxic conditions may be very similar to rates of oxic degradation (Henrichs and Reeburgh, 1987; Lee, 1992, and references therein).

Studies with pure cultures of bacteria support the contention that anaerobic degradation is not a priori slower than aerobic decomposition, but the pathways by which a given organic compound is degraded may be very different, particularly if mono- or dioxygenases are involved in the aerobic degradation pathway. For some pairs of substrates (catechol and resorcinol, for example), degradation of one compound is faster than the other under oxic conditions, but the second substrate is degraded more quickly than the first under anoxic conditions (Schink, 1989). For certain classes of organic compounds, 
such as saturated hydrocarbons, however, little to no degradation by pure cultures of bacteria occurs under anoxic conditions (Widdel, 1988).

\section{Bacterial Consortia}

From a microbiological perspective, the most profound difference between oxic and anoxic environments is the nature of the bacterial community. Because of their basic biochemical restrictions, anaerobic bacteria must often work in concert (usually referred to as consortia* ) to effect the complete remineralization of organic matter from complex substrates. One group of bacteria may perform an initial transformation of a substrate, and the products which are excreted are utilized by other groups of bacteria until the substrate carbon is completely oxidized to $\mathrm{CO}_{2}$ and $\mathrm{CH}_{4}$. Primary degraders are often dependent upon other bacteria to remove $\mathrm{H}_{2}$, for example, to make some fermentation reactions energetically possible (Schink, 1989).

Since few species of bacteria can be isolated in pure culture, and in any case pure culture studies are not intended to model the interactions of a natural bacteria community, laboratory application of a consortium approach provides valuable information which cannot be obtained by any other means. For laboratory consortia studies, an inoculum of bacteria from sediments or another source is enriched on a specific substrate. The bacteria which can grow most quickly and efficiently on the substrate are preferentially enriched, as are the secondary degraders which metabolize the products of the initial transformation reactions. Subsequent transfers of the initial enrichment decrease the percentage of bacteria which are not directly involved in the degradation pathway. Ultimately a physiologically stable assemblage of organisms is formed, which (in effect) can be treated as a 'pure culture' that catalyzes an efficient sequence of transformations to metabolize a specific substrate. A consortium of this nature can be manipulated (by selectively blocking a reaction or inhibiting growth of one type of organism, for example) to study the specific mechanisms and energetics of a degradation pathway.

This type of interdependent degradation process has been thoroughly investigated using consortia which convert benzoate to methane (Ferry and Wolfe, 1976). Among the other substrates used in bacterial consortia studies are methoxybenzoate (Balba et al., 1979), catechol (Balba and Evans, 1980b), ferulic acid (Healy et al., 1980), and aromatic

\footnotetext{
* The term "consortia" is used here to encompass both consortia (physiologically stable assemblages of bacteria catalyzing a specific sequence of transformations) and microbial food chains, in which one type of bacterium utilizes (for example) excretion products of another bacterium as described in the text.
} 
amino acids (Balba and Evans, 1980a). Many substrates used in consortia studies are structurally simple, however; a search of the literature revealed only two studies on the degradation of complex carbohydrate-containing material (lignocellulose) by enrichment cultures of aquatic bacteria (Benner et al., 1984a; Benner et al., 1984b). In addition, many consortia studies have been based on fresh-water enrichments, in which methanogens are the 'terminal' members of a consortium and a significant amount of substrate carbon is converted to methane, while few consortia studies have been based on sulfate reduction. A study of the transformation of cholesterol by a marine bacterial consortia is one of the few examples of this type of work (Taylor et al., 1981).

The extensive literature on pure cultures, co-cultures* , and consortia of rumen bacteria (i.e., bacteria enriched from the digestive system of ruminants such as cows) are a valuable source of information on the anaerobic degradation of carbohydrates (Hungate, 1966; Dehorty, 1967; Hungate, 1969; Miura et al., 1983; Hespell et al., 1987; Cotta, 1992; Malburg et al., 1992, for example). These studies elucidated the biophysical basis of anaerobic consortia, and have helped determine the rates and mechanisms of carbohydrate degradation by anaerobic bacteria. Although studies of rumen bacteria are an invaluable source of information, rumen consortia differ in important aspects from anaerobic consortia in marine sediments. The rumen is a natural bioreactor, in which the feedstock is chemically consistent (principally plant polysaccharides) and is fed in and washed out at a relatively constant rate. The organic matter reaching rumen anaerobes is always undegraded, and the system is chemically and thermally very stable. In addition, the terminal members of rumen consortia are methanogenic bacteria, whereas high levels of sulfate in marine systems results in the predominance of sulfate reducers as terminal members of marine bacterial consortia.

\section{Enzymes and Porins}

The degradation of a substrate by a bacterial consortium is determined by the net capacities of its members. For degradation of macromolecules, important determinants include the types and specificities of extracellular enzymes, and the nature and types of porins, expressed by the bacteria. In gram-negative bacteria (which include most marine bacteria), a lipopolysaccharide outer membrane surrounds the cell wall. Porins, trimeric proteins spanning the outer membrane, form the channels thorough which hydrophilic substrates are transported into the bacterial cell. Some porins are constituitive, while others, such as the

\footnotetext{
* Defined cultures with two species of bacteria.
} 
LamB maltoporin, are induced only in the presence of specific substrates (Benz, 1988). The uptake limit for "general diffusion" porins is approximately 600 daltons (equivalent to a trisaccharide) (Weiss et al., 1991), although some inducible porins have higher uptake limits. Substrates too large to pass through the porin channel must be hydrolyzed outside the cell by cell-surface or exoenzymes prior to ingestion.

Bacterial cell-surface and exoenzymes are specific for particular structural features (Priest, 1992). Polysaccharide-degrading exoenzymes generally can be divided into exoacting enzymes, which hydrolyze a polysaccharide from the nonreducing end, and endoacting enzymes, which hydrolyze a polysaccharide at random points within the polymer. Enzymes also have specificities for particular linkages, molecular weights, and structural configurations. One such enzyme is isoamylase, which can hydrolyze $\alpha(1,6)$ branches in glucose polysaccharides, but does not attack the $\alpha(1,6)$ linkages in pullulan, a linear glucose polymer (Antranikian, 1992). Another example is an amylolytic enzyme from Pyrococcus furiosus (a hyperthermophilic marine archaebacterium) which can hydrolyze amylopectin $(\alpha(1,4)$ glucose linkages with $\alpha(1,6)$ branches) and amylose $(\alpha(1,4)$ linkages) to produce mixtures of oligosaccharides, although maltose (glucose- $\alpha(1,4)$-glucose) and pullulan $(\alpha(1,6)$-linked maltotriose units) are not hydrolyzed by the enzyme (Koch $e t$ al., 1990). The consequences of enzyme and transport system specificities are reflected in studies of pure- and co-cultures of bacteria, which have demonstrated that bacteria preferentially utilize specific carbohydrates. Two examples of these pure- and co-culture studies have demonstrated preferential utilization of cellobiose over glucose (Bernier and Stutzenberger, 1987), and preferential utilization of select size ranges of xylan oligomers (Hespell et al., 1987).

\section{Previous Studies of Early Diagenesis}

Our limited ability to characterize macromolecular organic matter has severely hampered studies of early diagenesis. Because macromolecular organic matter cannot be structurally characterized in detail, approaches to indirectly derive information about the nature and types of reactions in marine sediments have been developed. One such approach involves determining overall carbon balance. The flux of carbon to sediments, fluxes of dissolved species out of the sediments, and burial of organic carbon are measured, and degradation rates and pathways are modeled. Using such an approach, Martens and coworkers (Martens et al., 1992, and references therein) determined that over 70\% of the particulate organic carbon at their Cape Lookout Bight site was not remineralized during early diagenesis. Carbon and nitrogen analyses suggested that the recalcitrant organic matter was 
probably derived from heavily degraded algal debris and nonwoody vascular plant tissue, but the specific characteristics which conferred resistance to degradation were unknown. In terms of Fig. 1.1, this approach measures sedimentation (A), upward flux of dissolved species (I), and burial of pore water $(\mathrm{L})$, while the transitions between the macromolecular particulate $\longrightarrow$ dissolved polymer $\longrightarrow$ dissolved monomer boxes $(\mathrm{C}, \mathrm{D}$, and $\mathrm{E})$ are unknowns.

A closer look at the energetics of these transitions, if not the actual organic matter transformations, has been provided by detailed studies of the dynamics of sulfur radiotracers (Jørgensen and Bak, 1991). By combining these measurements with determinations of porewater chemistry and bacterial numbers, rates of sulfate reduction, sulfur cycling budgets, and estimations of carbon cycling efficiency have been determined (Jorgensen et al., 1990). Microelectrodes have also been used to measure high-resolution time and space gradients of $\mathrm{O}_{2}, \mathrm{H}_{2} \mathrm{~S}$, and $\mathrm{SO}_{4}{ }^{2-}$ in surface sediments. This detailed picture of sulfate oxidation and reduction can be used to model carbon cycling dynamics (Gundersen and Jørgensen, 1990; Kuhl and Jørgensen, 1992).

The "G-model" type approach, as exemplified by Westrich and Berner (1984), uses the time course of radiolabeled $\mathrm{CO}_{2}$ evolution to characterize the substrate organic matter as consisting of a range of classes of degradability. Westrich and Berner found that their experiments with degradation of phytoplankton yielded two classes of organic matter which were remineralized, and one class of non-reactive organic matter. The "quality" of organic matter therefore has a measurable effect on degradation rates in sediment (see also (Emerson and Hedges, 1988; Boudreau and Ruddick, 1991), but the factors defining 'quality' which determine this range in degradability of organic matter have yet to be determined. The G-model approach makes distinctions in terms of degradability among and within the organic matter in the macromolecular particle $\longrightarrow$ dissolved polymer $\longrightarrow$ dissolved monomer boxes in Fig. 1.1, but the chemical nature of the different classes was not defined.

To look more closely at organic matter transformations and to determine the relative uptake of specific classes of organic matter, radiolabeled simple substrates have been added to sediments, and the evolution of labeled endproducts monitored (Buscail, 1986). This type of study corresponds to the dissolved monomer $\rightarrow$ total $\mathrm{CO}_{2}$ transition (F) of Fig. 1.1. Hydrolytic activities in sediments have also been monitored using fluorescent substrate analogs (King, 1986; Meyer-Reil, 1987, for example). While small substrates have been used extensively as proxies for larger polymers (Henrichs, 1992; Lee, 1992), their suitability as models of polymer uptake and macromolecular degradation is uncertain. In an effort to address this problem, whole labeled organisms such as bacteria (Novitsky, 1986) 
or phytoplankton (Henrichs and Doyle, 1986) have been added to sediment samples, and evolution of radiolabeled end products has been monitored. The distribution of label within the organisms was unknown, however, and carbon recoveries were often difficult to quantify. In general, degradation in sediments, particularly of larger substrates, has not been adequately examined because the systems studied have on the whole been overly complex and poorly characterized, and substrates were either inadequately characterized or were structurally too simple to be useful in determining the major factors influencing macromolecular degradation.

\section{Marine Carbohydrates}

\section{Terminology and General Structures of Carbohydrates}

A brief review of carbohydrate terminology and structure, and of previous work on marine carbohydrates, is necessary in order to demonstrate the types of structural information which are missing from previous studies of marine carbohydrates, and to set the scene for the discussion of NMR spectroscopy in Chapter 2, phytoplankton carbohydrates in Chapter 3 , and the bacterial degradation of carbohydrates in Chapters 4 and 5.

Carbohydrates are 'hydrates of carbon' of the general form $\left(\mathrm{C}\left(\mathrm{H}_{2} \mathrm{O}\right)\right)_{\mathrm{n}}$. A simple sugar (or monosaccharide) typically consists of a five- or six-membered ring, whose constituent carbons are numbered as shown in Fig. 1.2. The \#1 carbon (the anomeric carbon) is particularly important, because it is a key linkage site for practically every type of carbohydrate. The hydroxyl group and proton attached to the anomeric carbon can take two different orientations: a $\beta$-carbohydrate is formed when the hydroxyl group is above the plane of the ring; an $\alpha$-carbohydrate is formed when the \#1 hydroxyl group is below the plane of the ring (Fig. 1.2b,c).

The neutral monosaccharides differ from one another principally in the orientation of the \#2, \#3, and/or \#4 hydroxyl groups. Glucose and galactose, for example, differ solely in the orientation of the \#4 hydroxyl group. Fig. 1.3 shows three general groups of monosaccharides commonly found in the marine environment: the hexamers (glucose, galactose, and mannose), the deoxy sugars (fucose and rhamnose) which lack a hydroxyl group at the \#6 carbon, and the pentamers (xylose, ribose, and arabinose), which do not have a \#6 carbon. Two other major classes of carbohydrates which will not be discussed at length are uronic acids, which have a carboxyl group at the \#6 carbon, and amino sugars, in which a $-\mathrm{NH}_{2}$ group substitutes for a hydroxyl group at either the \#2, \#3, \#4, or \#6 positions. Carbohydrates may also have sulfate groups at one of these positions. The \#1 hydroxyl group can link one monosaccharide to another or to non-sugar 
components. Two monosaccharides linked together in this fashion are known as a disaccharide; any carbohydrate in the range of two to ten monosaccharide units is generally known as an oligosaccharide, and carbohydrates of more than ten units are known of polysaccharides (Fig 1.4). There are a wide range of carbohydrate linkage possibilities; the anomeric carbon of one monosaccharide can be linked to the $\# 2$, \#3, \#4, or \#6 carbon of another monosaccharide.

(a)

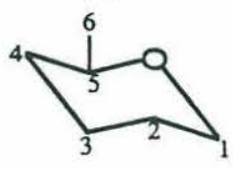

(b)

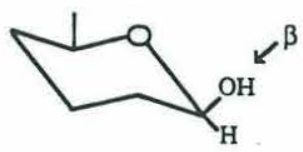

(c)

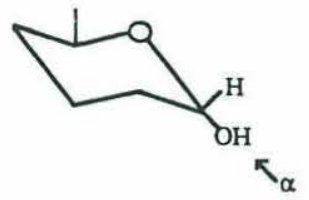

Fig. 1.2

Monosaccharide structure: carbon and protons are labeled 1-6 as shown (a), $\beta$-anomer (b), $\alpha$-anomer (c).

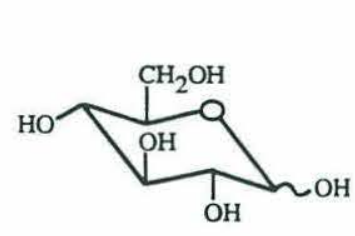

glucose

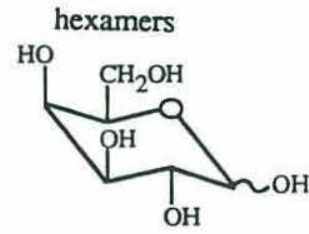

galactose

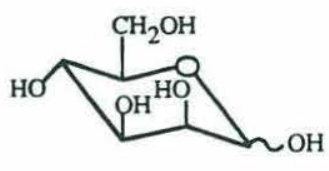

mannose

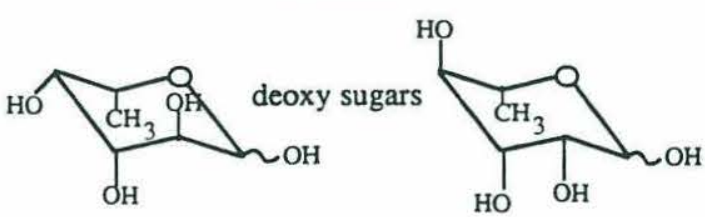

fucose

rhamnose
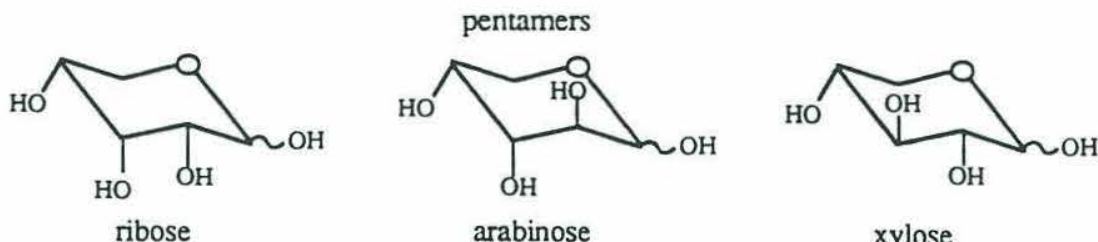

xylose

Fig. 1.3

The eight neutral monosaccharides most commonly found in marine systems. 

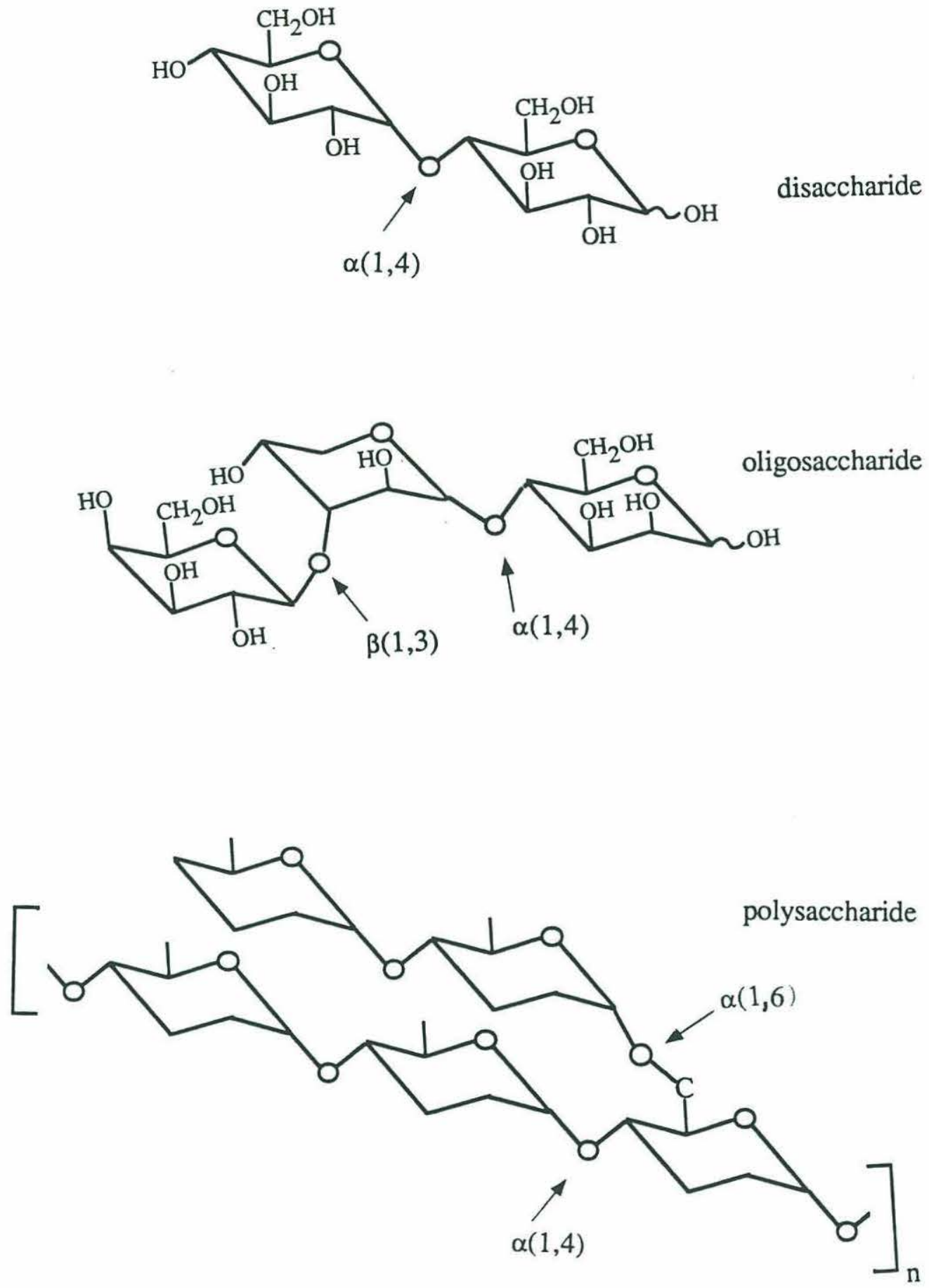

Fig. 1.4

Examples of a disaccharide, an oligosaccharide, and a polysaccharide. (Chemical and anomeric linkages are marked with arrows). 
Two different monosaccharides can be linked together in any of 16 possible combinations; three different monosaccharides have at least 384 linkage possibilities. Because of the spatial restrictions imposed by these linkages, oligo- and polysaccharides also can have tertiary structures, such as ribbons, loops, or sheets, which determine their hydrodynamic volume in solution, and affect properties such as solubility and relative lability to acid or enzymatic degradation. Cellooligosaccharides, for example, are much less soluble than maltooligosaccharides, although they differ only in anomeric linkage: cellobiose is glucose$\beta(1,4)$-glucose, whereas maltobiose is glucose- $\alpha(1,4)$-glucose. Hydrolytic enzymes such as $\alpha$-amylase are, in a similar fashion, specific only for $\alpha(1,4)$ linkages, and do not cleave $\beta(1,4)$ anomeric linkages.

\section{Analysis of Marine Carbohydrates}

As briefly outlined above, there is a wide variety of structural possibilities among carbohydrates; while the basic building blocks are quite similar to one another, the structures which can be built from these pieces are extremely varied. Analysis of these structural distinctions can be difficult, however, since isolation of carbohydrates from solution or from an organic matrix is not a trivial problem, and determination of carbohydrate structure typically involves a further array of analytical difficulties. Recovery of carbohydrates from natural samples is strongly influenced by the organic and inorganic composition of the matrix in which the carbohydrates are found, as indicated by the significantly higher variability in reproducibility of hydrolysis/derivitization procedures among replicate analyses of sediment and plankton as compared to standards (Walters and Hedges, 1988).

Since choice of extraction and analytical techniques can affect recovery of carbohydrates (Mopper, 1977; Dawson and Mopper, 1978), most reports represent a minimum of the carbohydrate components, in both qualitative and quantitative terms. Even measurement of total carbohydrates is uncertain, since the response of the most commonly used colorimetric 'total' carbohydrate determination procedures, such as the phenol-sulfuric acid method (Chaplin and Kennedy, 1986), depends strongly on monomer composition of the sample (Burney and Sieburth, 1977; Johnson and Sieburth, 1977). Principally because of these analytical problems, most studies of marine carbohydrates have been limited to determinations of eight or nine of the most common neutral monosaccharides (Handa and Yanagi, 1969; Cowie and Hedges, 1984; Ittekkot et al., 1984; Tanoue and Handa, 1987; Hamilton and Hedges, 1988; Cowie, 1990; Cowie et al., 1992; Handa et al., 1992).

Samples are typically analyzed by acid hydrolysis, derivatization, and gas- 
chromatography or gas-chromatography-mass spectrometry (GC-MS), yielding information about monomer composition and concentration. The focus on monomer composition means that a tremendous amount of potentially important structural information is lost. Studies of the bacterial degradation of carbohydrates, as well as the literature on carbohydrate biochemistry (Bishop and Jennings, 1982; Miller et al., 1992; Pimenta et al., 1992; Weis et al., 1992; Borman, 1993), demonstrate that structural differences among carbohydrates determined in part by size, branching, linkage point, and orientation are key factors which affect reactivity and functionality in macromolecules such as polysaccharides, glycoproteins, and lipopolysaccharides.

The following section includes a brief summary and comparison of the monosaccharide concentration and composition of marine organisms, and dissolved, particulate, and sedimentary carbohydrates.

\section{Carbohydrates of Marine Organisms}

Phytoplankton serve as a principle source for carbohydrates found in seawater, particles, and sediments; the photosynthetic conversion of $\mathrm{CO}_{2}$ to biomass is the basis of carbohydrate production. The carbohydrate composition of marine phytoplankton and a marine cyanobacterium is discussed at greater length in Chapter 3, and Appendix B contains an extensive compilation of literature on phytoplankton carbohydrates.

The carbohydrate content of marine organisms varies greatly; in brown and red algae, carbohydrates may comprise up to $74 \%$ of total organic matter (Romankevich, 1984), while planktonic algae have a carbohydrate content generally ranging from 20 to 40\% (Parsons et al., 1961), and zooplankton carbohydrate content (on a carbon-normalized basis) is 2-4 times lower than that of phytoplankton (Hamilton and Hedges, 1988). Phytoplankton carbohydrate composition has been surveyed in both field samples and laboratory monocultures. In general, glucose has been found to be the most common monosaccharide, (Hecky et al., 1973; Cowie and Hedges, 1984; Tanoue and Handa, 1987; Hamilton and Hedges, 1988; Cowie, 1990; Cowie et al., 1992), probably due to the fact that phytoplankton storage carbohydrates are primarily composed of glucose (Lee, 1980).

\section{Dissolved Carbohydrates}

Dissolved carbohydrates can be derived from a variety of sources, including phytoplankton excretion, cell lysis, grazing by zooplankton, enzymatic action on particles and detritus, and bacterial degradation of organic matter. Dissolved carbohydrates have been reported in 
the range of tens to hundreds of micrograms per liter (Vaccaro and Jannasch, 1966; Johnson and Sieburth, 1977; Mopper, 1977; Liebezeit et al., 1980; Ittekkot et al., 1981; 1982; Sakugawa and Handa, 1985), again with glucose as the most common monosaccharide.

A number of studies have highlighted the fact that concentrations of dissolved carbohydrates can vary significantly on short timescales (Burney and Sieburth, 1977; Harvey et al., 1986), so reports indicating large variability in carbohydrate composition and concentration, especially in surface waters and in highly productive regions, may reasonably represent a dynamic situation. This hypothesis is supported by data discussed in Chapters 4 and 5, in which very rapid degradation of some oligo- and polysaccharides in bacterial cultures was measured.

Dissolved carbohydrates occur as monosaccharides, oligosaccharides, and polysaccharides, although few reports of carbohydrate concentrations distinguish between these pools. Ittekkot (1981) found that "combined carbohydrates" (total carbohydrates monosaccharides) comprised the majority of the total dissolved carbohydrates detected during a phytoplankton bloom. Monosaccharide concentrations were relatively uniform throughout the bloom. Relatively constant concentrations of monosaccharides as compared to dissolved polysaccharides were also found by Burney (1977), who also suggested that the monosacchrides may be at or near uptake thresholds for bacteria.

Glucose dominated the monosaccharides and was the predominant constituent of oligosaccharides and dissolved polysaccharides isolated from surface and deep waters of the North Pacific (10 and $2500 \mathrm{~m}$ ) and Bering Sea $(10 \mathrm{~m}$ and $2000 \mathrm{~m}$ ) (Sakugawa et al., 1990). Concentrations of all three classes of carbohydrates were in the range of tens of micrograms per liter of seawater, and in constrast to the studies mentioned previously, monosaccharides were found to have the greatest range in values. Oligosaccharides identified by derivitization and chemical ionization- and electron-impact mass spectrometry included sucrose, trehalose, melibiose, and several unidentified disaccharides. Some of the polysaccharides were found to contain uronic acids, and a heteropolysaccharide was also found in shallow water but not in deep water samples (Sakugawa and Handa, 1985). The connection between dissolved and particulate carbohydrate pools was highlighted by the identification of a range of similar low molecular weight oligosaccharides in particles and cultures of phytoplankton. Low molecular weight dissolved carbohydrates found at depth may have originated from phytoplankton components of rapidly sinking particles.

Similarities were also found between dissolved and particulate polysaccharides isolated from Mikawa Bay, Japan, during a dinoflagellate bloom. A branched heteropolysaccharide of molecular weight $>4000$ daltons which reportedly contained 
sulfate half-esters, as well as a glucan with $\beta(1,3)$ and $\beta(1,6)$ linkages, were isolated from concentrates of seawater. Boiling water extracts of particulate matter contained a similar heteropolysaccharide and a $\beta$-glucan; extracellular release and/or cell lysis may have produced the dissolved polysaccharides (Sakugawa and Handa, 1985).

\section{Particulate Carbohydrates}

Particulate carbohydrates are generally attributed to detrital marine organic matter, colonizing bacteria, and (depending on sampling location) terrigenous plant material. Glucose is again the most abundant monosaccharide identified in most studies of marine particles, including those collected in sediment traps from Dabob Bay (Cowie and Hedges, 1984); Saanich Inlet (Hamilton and Hedges, 1988), the Sargasso Sea (Ittekkot et al., 1984), and polar regions (Ittekkot and Degens, 1982; Liebezeit, 1984), the Bering Sea and North Pacific (Tanoue and Handa, 1987). These same studies found that carbohydrates typically accounted for $2-15 \%$ of total particulate organic matter. Sample processing can affect these results, however; Ittekkot (1984) found that $18 \%$ of the carbohydrates originally present as particulate matter were transferred to the dissolved pool during processing.

Differences in composition and relative lability have been found among different fractions of carbohydrate extracts. In sinking particles collected by sediment trap near Antarctica, glucose was usually the most common monosaccharide in particles with a high percentage of water-extractable carbohydrates, while samples with relatively higher fractions of base-extractable and residual carbohydrates were sometimes dominated by galactose, mannose, or arabinose (Handa et al., 1992). For particles collected by sediment trap in the northwest Pacific, water-extractable carbohydrates decreased faster with depth than did other organic components, while insoluble carbohydrates were less reactive than other fractions of organic matter (Handa and Yanagi, 1969).

Few studies of particles determined any information beyond monomer compositon. Handa and Yanagi (1969), however, observed that $1 / 3$ of total particulate carbohydrate was water-soluble and composed primarily of glucose, probably with 1,3 linkages, while the remaining insoluble $2 / 3$ consisted of a mixture of glucose, galactose, mannose, xylose, and glucuronic acid with 1,2 or 1,4 linkages. The composition and relative size (expressed as D.P., degree of polymerization=number of monosaccharide residues in a polysaccharide) of the water-insoluble carbohydrates isolated from particles was relatively constant with depth; while the concentration of hot-water soluble glucan decreased significantly with depth. 
Interpretations of the carbohydrate composition of particles is complicated by variability among particles. The monosaccharide composition of sinking particles collected in sediment traps, and suspended particles collected with filters, for example, was found to differ significantly (Tanoue and Handa, 1987). Seasonal variations in particle sources, and sediment resuspension for deep traps, may also account for differences in particle composition (Cowie and Hedges, 1984). In addition, bacterial production of carbohydrates in unpoisoned traps probably occurs simultaneously with degradation of more labile components. A relatively constant total carbohydrate concentration may mask changes in composition and structure, during which more labile phytoplankton carbohydrates (storage polysaccharides, for example) might be converted to bacterial biomass, which would include relatively resistant cell-wall carbohydrates.

\section{Carbohydrates in Sediments}

Sinking particles are a major source of carbohydrates to marine sediments. Benthic organisms and bacteria degrade particulate organic matter, remineralizing it to $\mathrm{CO}_{2}$ and synthesizing cellular components including carbohydrates. The intertwined production and degradation processes, coupled with additional complications such as resuspension, bioturbation, and seasonal variation in particle source and abundance, result in a complex pattern of carbohydrates in marine sediments.

As previously discussed, extraction and derivitization procedures have a significant influence on recovery of carbohydrates, so the reported carbohydrate contribution to sediment organic carbon should be regarded as a minimum. Extractable and characterizable carbohydrates were ca. 2-6\% of total sedimentary organic carbon in sediments from Dabob Bay (Washington) (Cowie and Hedges, 1984), the equatorial eastern Atlantic (Moers et al., 1990), and the Namibian Shelf (Klok et al., 1984b). Carbohydrate contents of reducing sediments of Saanich Inlet and the Black Sea were considerably higher, in the range of 1522\% (Mopper, 1977; Hamilton and Hedges, 1988). Differences were attributed to a range of factors, including organic matter sources, water column depth and transformation processes, and activity of benthic macrofauna. Reports of carbohydrate concentrations in sediments may also represent a minimum, since characterization of sedimentary organic matter is difficult. Klok et al. (1984b), for example, found that only $22 \%$ of sedimentary organic carbon could be classified as amino acids, carbohydrates, volatile fatty acids, or lipids. The $4 \%$ contribution of carbohydrates therefore represents almost $20 \%$ of identified organic carbon. Pyrolysis-mass spectrometry of the unidentified organic carbon released during the extraction procedure produced spectra which were attributed to carbohydrate 
alteration products; Klok and co-workers concluded that approximately $22 \%$ of sedimentary organic carbon actually originated from carbohydrates.

Glucose is the most commonly reported monosaccharide from most sites (Mopper, 1977; Cowie and Hedges, 1984; Tanoue and Handa, 1987), although comparably high concentrations of galactose and mannose have been found at some sites (Yamaoka, 1983; Klok et al., 1984b; Moers et al., 1990). A few studies have looked beyond the major neutral monosaccharides. A large suite of minor sugars, attributed to bacteria and comprising 5-15\% of total sugars, have also been identified (Klok et al., 1984a; Klok et al., 1984b; Moers et al., 1990).

Depth trends of carbohydrate composition and concentration in sediments are often difficult to interpret. While total carbohydrates decrease with depth in samples from some sites (Hamilton and Hedges, 1988), other sites show little or no concentration changes with depth (Cowie and Hedges, 1984; Tanoue and Handa, 1987; Moers et al., 1990). Even for cases where total carbohydrates decrease with depth, relative composition is usually invariant, and carbohydrate content never decreases to zero. Residual carbohydrates which are apparently resistant to further degradation have even been found in 2 million year old sediments (Whelan and Emeis, 1992).

Due to the problems associated with the extraction of carbohydrates from a sediment matrix, determining structural information about sediment carbohydrates is very difficult, and has not been attempted frequently. Klok et al. (1984b) used gel permeation chromatography to divide extractable, soluble carbohydrates into three size classes $(<400$ daltons, $400-2000$ daltons, $>2000$ daltons). Approximately $10 \%$ of the total initial carbohydrates were water extractable, and a significant fraction were in the $>2000$ dalton size fraction. Gel permeation chromatography was also used to separate carbohydrates in humic and fulvic acids extracted from sediments in Hiroshima Bay (Yamaoka, 1983). Carbohydrate concentrations in fulvic acids were much greater than in humic acids, and most of the carbohydrates were found in the $>1500$ dalton fraction.

\section{Summary: Implications for the Degradation of Marine Carbohydrates}

The analysis of carbohydrates in marine samples is subject to many complications; the net result is that in most cases, little is known beyond total neutral carbohydrate concentration and monomer composition of a given sample. Carbohydrates in seawater, particles, and sediments are composed of a suite of monosaccharides, of which glucose is usually the most common. Concentrations of individual monosaccharide, and often of total carbohydrates, vary irregularly with depth in the water column and sediments. Since 
carbohydrate structure and reactivity are controlled in part by aspects of structure which have not been determined (size, linkage position, anomeric linkage), however, a large portion of the marine carbohydrate picture remains obscured.

A comparison of the literature on marine carbohydrates and on bacterial degradation of carbohydrates highlights a question about carbohydrates in marine systems. Studies of marine organisms have shown that phytoplankton produce a range of carbohydrates, with varying monosaccharide composition (Parsons et al., 1961; Hecky et al., 1973; Lee, 1980). Microbiological studies of pure cultures of bacteria have demonstrated that bacterial enzyme and transport systems have precise specificities for selected carbohydrates (Bernier and Stutzenberger, 1987; Hespell et al., 1987). The fact that bacteria have a demonstrated preference for certain carbohydrates implies that specific carbohydrates will be preferentially utilized in marine environments. Studies of marine organisms, particles, and sediments, however, particularly the comprehensive studies of Cowie $(1984 ; 1990 ; 1992)$, Tanoue (1987), and Hamilton (1988), have shown that there are no systematic differences in monosaccharide compositions between marine phytoplankton, particles, and sediments.

Although phytoplankton are a major source of particle and sedimentary carbohydrates, and diagenesis in the water column and sediments would be expected to affect relative carbohydrate composition, profiles of particles in the water column and of sediment cores show no regular trends in carbohydrate composition. Carbohydrate depth profiles in sediments (Cowie and Hedges, 1984; Hamilton and Hedges, 1988) in fact showed that a significant fraction of monosaccharides apparently belong to the fraction of organic matter which is relatively resistant to degradation.

A lack of systematic differences in monosaccharide composition, however, does not mean that the carbohydrates in plankton, particles, and sediments are truly uniform in structure. Lack of systematic variation in monosaccharide composition may obscure large variations in carbohydrate structure or matrix. Klok et al. (1984b), for example, found that the monomer composition of total, extractable, and residual carbohydrates from sediments was very similar. The difference in techniques needed to extract these carbohydrates, however, suggests that the manner in which the carbohydrates are linked, or the structures in which they are found, are very different. A significant fraction of carbohydrates were identified only through the use of pyrolysis-mass spectrometry, again suggesting that some monomers were tightly bound in a matrix. The reason for the lack of systematic variations in monosaccharide composition of marine plankton, particles, and sediments, and the relative similarity of monosaccharides down a sediment core, may therefore lie in the structure of the parent oligo- and polysaccharides. The carbohydrates which are preserved in marine sediments may be linked differently than carbohydrates (made of the same 
monosaccharides) which are degraded within the upper layer of the sediments.

\section{Thesis goal: A New Approach to Early Diagenesis}

The 'link' between carbohydrate structure and reactivity is the central theme of this thesis: what are the rate determining factors in the bacterial degradation of macromolecular carbohydrates? In terms of Fig. 1.1, the transitions between the dissolved polymer $\rightarrow$ dissolved monomer boxes, and the dissolved monomer $\rightarrow$ total $\mathrm{CO}_{2}$ boxes, are the major focus. Specifically, the goal is to determine the effects of structural features of carbohydrates such as monomer composition, size, linkage position, and anomeric linkage on the rate and manner by which they are degraded by mixed cultures of anaerobic marine bacteria.

As outlined above, previous geochemical studies of organic matter degradation and microbiological studies of degradation of specific substrates have provided important information about early diagenesis in marine sediments. Both types of studies suffer from serious limitations, however, and to overcome these problems it is necessary to apply an integrated chemical and microbiological approach to study the dynamics of carbon cycling in marine systems. We have reached the point where neither approach alone is adequate to address complex issues such as the factors which determine whether an organic structure will be degraded, the nature and extent of organic matter preservation in a given environment, or the response of microorganisms to new substrates.

The work presented in this thesis represents an initial attempt to apply a combined chemical and microbiological approach. The major features are a well-characterized system in which substrate, intermediates, and products can be identified and quantified, and a natural mixed microbial population which approximates the plasticity of bacterial consortia in marine sediments. By enriching bacteria from sulfate-reducing sediments on strictlycontrolled carbon sources, reproducible model systems were obtained with which to study the degradation of chemically well-defined substrates. Headspace gases $\left(\mathrm{CO}_{2}, \mathrm{H}_{2}, \mathrm{CH}_{4}\right.$, $\mathrm{H}_{2} \mathrm{~S}$ ) were measured by gas chromatography, and the carbohydrate substrate and carbohydrate intermediates were separated and quantified via gel permeation chromatography and high-pressure liquid chromatography. Specific structural alterations in the substrate were determined using NMR spectroscopy. The flow of carbon from substrates through to end-products was thereby followed quantitatively. By systematically varying structural features of substrates in replicate cultures, the effects of specific structural features on degradation rates and patterns were determined. 
In order to successfully use this approach, a number of concrete experimental requirements first had to be met:

(1) establish a reproducible, quantitative culture system to study the degradation of well-characterized substrates

(2) develop analytical techniques to quantify carbohydrates and their degradation products

(3) apply NMR spectroscopy to the detailed structural characterization of carbohydrates and their degradation products in order to determine specific structural features

Chapter Two provides the necessary background and development with NMR spectroscopy, and in Chapter Three NMR spectroscopy is applied to the characterization of carbohydrates from several marine plankton as a first effort at determining macromolecular carbohydrate structure of marine organisms. Chapter Four presents the results of an exhaustive series of culture experiments which demonstrated the reproducible and fundamentally robust nature of the culture system. The culture system, analytical techniques, and NMR spectroscopy are combined in Chapter Five to study the degradation of polysaccharides by anaerobic marine bacteria. The work is summarized in Chapter Six; suggestions for future work by those with high energy and low sleep requirements are also included. 


\section{References}

Antranikian, G. (1992). Microbial degradation of starch. Microbial Degradation of Natural Products. New York, VCH. 27-56.

Azam, F., T. Fenchel, J. G. Field, J. S. Gray, L. A. Meyer-Reil and F. Thingstad (1983). The ecological role fo water-column microbes in the sea. Mar. Ecol. Prog. Ser. 10: 257263.

Balba, M. T., N. A. Clarke and W. C. Evans (1979). The methanogenic fermentation of plant phenolics. Biochem. Soc. Trans. 7: 1115-1116.

Balba, M. T. and W. C. Evans (1980a). Methanogenic fermentation of the naturally occurring aromatic amino acids by a microbial consortium. Biochem. Soc. Trans. 8: 625627.

Balba, M. T. and W. C. Evans (1980b). The methanogenic biodegradation of catechol by a microbial consortium: evidence for the production of phenol through cis-benzenediol. Biochem. Soc. Trans. 8: 452-453.

Benner, R., A. E. MacCubbin and R. E. Hodson (1984a). Preparation, characterization, and microbial degradation of specifically radiolabeled ${ }^{14} \mathrm{C}$-lignocelluloses from marine and freshwater macrophytes. Appl. Environ. Microbiol. 47(2): 381-389.

Benner, R., A. E. MacCubbin and R. E. Hodson (1984b). Anaerobic biodegradation of the lignin and polysaccharide components of lignocellulose and synthetic lignin by sediment microflora. Appl. Environ. Microbiol. 47(5): 998-1004.

Benz, R. (1988). Structure and function of porins from gram-negative bacteria. Ann. Rev. Microbiol. $1988:$ 359-393.

Bernier, R. and F. Stutzenberger (1987). Preferential utilization of cellobiose by Thermomonospora curvata. Appl. Environ. Microbiol. 53(8): 1743-1747.

Bishop, C. T. and H. J. Jennings (1982). Immunology of polysaccharides. The Polysaccharides. New York, Academic Press. 292-325.

Borman, S. (1993). Chemical engineering focuses increasingly on the biological. Chem. Eng. News (Jan. 11, 1993): 26-36.

Boudreau, B. P. and B. R. Ruddick (1991). On a reactive continuum representation of organic matter diagenesis. Am. J. Sci. 291: 507-538.

Burdige, D.J. and C.S. Martens (1988). Biogeochemical cycling in an organic-rich coastal marine basin: 10. The role of amino acids in sedimentary carbon and nitrogen cycling. Geochim. Cosmochim. Acta 52: 1571-1584.

Burney, C. M. and J. M. Sieburth (1977). Dissolved carbohydrates in seawater. II. A spectrophotometric procedure for total carbohydrate analysis and polysaccharide estimation. Mar. Chem. 5: 15-28.

Buscail, R. (1986). Biogeochemical processes of incorporation, transformation and migration of organic matter at the marine water-sediment interface: Simulation by ${ }^{14} \mathrm{C}$ 
labelled compounds. Org. Geochem. 10: 1091-1097.

Chaplin, M. F. and J. F. Kennedy (1986). Carbohydrate Analysis: A Practical Approach. Oxford, IRL Press.

Cotta, M. A. (1992). Interaction of ruminal bacteria in the production and utilization of maltooligosaccharides from starch. Appl. Env. Microbiol. 58(1): 48-54.

Cowie, G. L. (1990). Marine Organic Diagenesis: A Comparative Study of Amino Acids, Neutral Sugars, and Lignin. 189. University of Washington.

Cowie, G. L. and J. I. Hedges (1984). Carbohydrates sources in a coastal marine environment. Geochim. Cosmochim. Acta 48: 2075-2087.

Cowie, G. L., J. I. Hedges and S. E. Calvert (1992). Sources and reactivities of amino acids, neutral sugars, and lignin in an intermittently anoxic marine environment. Geochim. Cosmochim. Acta 56: 1963-1978.

Dawson, R. and K. Mopper (1978). A note on the losses of monosaccharides, amino sugars, and amino acids from extracts during concentration procedures. Analyt. Biochem. 84: $186-190$.

Dehorty, B. A. (1967). Rates of isolated hemicellulose degradation and utilization by pure cultures of cellulolytic rumen bacteria. J. Bacteriol. 89: 1515-1520.

Emerson, S. (1985). Organic carbon preservation in marine sediments. The Carbon Cycle and Atmospheric CO2: Natural Variations Archean to the Present. 78-87.

Emerson, S. and J. I. Hedges (1988). Processes controlling the organic carbon content of open ocean sediments. Paleoceanog. 3(5): 621-634.

Ferry, J. G. and R. S. Wolfe (1976). Anaerobic degradation of benzoate to methane by a microbial consortium. Arch. Microbiol. 107: 33-40.

Fuhrman, J. A. and F. Azam (1982). Thymidine incorporation as a measure of heterotrophic bacterioplankton production in marine surface waters: evaluation and field results. Mar. Biol. 66(2): 109-120.

Fuhrman, J. A., K. McCallum and A. A. Davis (1992). Novel major archaebacterial group from marine plankton. Nature 356: 148-149.

Gocke, K., R. Dawson and G. Liebezeit (1981). Availability of dissolved free glucose to heterotrophic microorganisms. Mar. Biol. 62: 209-216.

Gottschalk, G. (1986). Bacterial Metabolism. Berlin, Springer-Verlag.

Gundersen, J. K. and B. B. Jørgensen (1990). Microstructure of diffusive boundary layers and the oxygen uptake of the sea floor. Nature 345: 604-607.

Hamilton, S. E. and J. I. Hedges (1988). The comparative chemistries of lignins and carbohydrates in an anoxic fjord. Geochim. Cosmochim. Acta 52: 129-152.

Handa, N., T. Nakatsuka, M. Fukuchi, H. Hattori and T. Hoshiai (1992). Vertical fluxes and ecological significance of organic materials during the phytoplankton bloom during 
austral summer in Breid Bay, Antarctica. Mar. Biol. 112: 469-478.

Handa, N. and K. Yanagi (1969). Studies on water-extractable carbohydrates of the particulate matter from the northwest Pacific Ocean. Mar. Biol. 4: 197-207.

Harvey, H. R., R. D. Fallon and J. S. Patton (1986). The effect of organic matter and oxygen on the degradation of bacterial membrane lipids in marine sediments. Geochim. Cosmochim. Acta 50: 795-804.

Healy, J. B., Jr., L. Y. Young and M. Reinhard (1980). Methanogenic decomposition of ferulic acid, a model lignin derivative. Appl. Environ. Microbiol. 39: 436-444.

Hecky, R. E., K. Mopper, P. Kilham and E. T. Degens (1973). The amino acid and sugar composition of diatom cell-walls. Mar. Biol. 19: 323-331.

Hedges, J. I. (1992). Global biogeochemical cycles: progress and problems. Marine Chem. 39: 67-93.

Heitkamp, M. A., W. Franklin and C. E. Cerniglia (1988). Microbial metabolism of polycyclic aromatic hydrocarbons: isolation and characterization of a pyrene-degrading bacterium. Appl. Environ. Microbiol. 54(10): 2549-2555.

Henrichs, S. M. (1992). Early diagenesis of organic matter in marine sediments: progress and perplexity. Mar. Chem. 39: 119-149.

Henrichs, S. M. and A. P. Doyle (1986). Decomposition of ${ }^{14} \mathrm{C}$-labeled organic substances in marine sediments. Limnol. Oceanogr. 31(4): 765-778.

Henrichs, S. M. and W. S. Reeburgh (1987). Anaerobic mineralization of marine sediment organic matter: rates and the role of anaerobic processes in the oceanic carbon economy. Geomicrobiol. J. 5(3/4): 191-237.

Hespell, R. B., R. Wolf and R. J. Bothast (1987). Fermentation of xylans by Butyrivibrio fibrisolvens and other ruminal bacteria. Appl. Environ. Microbiol. 53(12): 2849-2853.

Hungate, R. E. (1966). The Rumen and Its Microbes. New York, Academic Press.

Hungate, R. E. (1969). A roll tube method for cultivation of strict anaerobes. Methods in Microbiology. New York, Academic Press.

Ittekkot, V., U. Brockmann, W. Michaelis and E. T. Degens (1981). Dissolved free and combined carbohydrates during a phytoplankton bloom in the northern North Sea. Mar. Ecol. Prog. Ser. 4: 299-305.

Ittekkot, V. and E. T. Degens (1982). Monosaccharide composition of acid-hydrolyzable carbohydrates in particulate matter during a plankton bloom. Limnol. Oceanogr. 27(4): 770-776.

Ittekkot, V., W. G. Deuser and E. T. Degens (1984). Seasonality in the fluxes of sugars, amino acids, and amino sugars to the deep ocean: Sargasso Sea. Deep Sea Res. 31(9): 1057-1069.

Johnson, K. M. and J. M. Sieburth (1977). Dissolved carbohydrates in seawater. I. A 
precise spectrophotometric analysis for monosaccharides. Mar. Chem. 5(1): 1-13.

Jørgensen, B. B. and F. Bak (1991). Pathways and microbiology of thiosulfate transformations and sulfate reduction in a marine sediment (Kattegat, Denmark). Appl. Environ. Microbiol. 57(3): 847-856.

Jørgensen, B. B., M. Bang and T. H. Blackburn (1990). Anaerobic mineralization in marine sediments from the Baltic Sea-North Sea transition. Mar. Ecol. Prog. Ser. 59: 3954.

King, G. M. (1986). Characterization of $\beta$-glucosidase activity in intertidal marine sediments. AEM 51(2): 373-380.

Klok, J., M. Baas, H. C. Cox, J. W. De Leeuw, W. I. C. Rijpstra and P. A. Schenck (1984b). Qualitative and quantitative characterization of the total organic matter in a recent marine sediment (Part II). Org. Geochem. 6: 265-278.

Klok, J., H. C. Cox, M. Baas, P. J. W. Schuyl, J. W. de Leeuw and P. A. Schenck (1984a). Carbohydrates in recent marine sediments I. origin and significance of deoxy- and o-methyl-monosaccharides. Org. Geochem. 7: 73-84.

Koch, R., P. Zablowski, A. Spreinat and G. Antianikian (1990). Extremely thermostable amylolytic enzyme from the archaebacterium Pyrococcus furiosus. FEMS Microbiol. Lett. 71: $21-26$.

Kuhl, M. and B. B. Jørgensen (1992). Microsensor measurements of sulfate reduction and sulfide oxidation in compact microbial communities of aerobic biofilms. Appl. Environ. Microbiol. 58(4): 1164-1174.

Lee, C. (1992). Controls on organic carbon preservation: the use of stratified water bodies to compare intrinsic rates of decomposition in oxic and anoxic systems. Geochim.

Cosmochim. Acta 56: 3323-3335.

Lee, R. E. (1980). Phycology. Cambridge, Cambridge University Press.

Liebezeit, G. (1984). Particulate carbohydrates in relation to phytoplankton in the euphotic zone of the Bransfield Strait. Polar Biol. 2: 225-228.

Liebezeit, G., M. Bolter, I. F. Brown and R. Dawson (1980). Dissolved free amino acids and carbohydrates at pycnocline boundaries in the Sargasso Sea and related microbial activity. Oceanol. Acta 3(3): 357-362.

Malburg, L. M., J. M. Tamblyn Lee and C. W. Forsberg (1992). Degradation of cellulose and hemicellulose by rumen microorganisms. Microbial Degradation of Natural Products. New York, VCH. 127-159.

Martens, C. S., R. I. Haddad and J. P. Chanton (1992). Organic matter accumulation, remineralization, and burial in an anoxic coastal sediment. Organic Matter: Productivity, Accumulation, and Preservation in Recent and Ancient Sediments. New York, Columbia University Press. 82-98.

Meyer-Reil, L.-A. (1987). Seasonal and spatial distribution of extracellular enzymatic activities and microbial incorporation of dissolved organic substrates in marine sediments. Appl. Environ. Microbiol. 53(8): 1748-1755. 
Meyer-Reil, L. A. (1978). Uptake of glucose by bacteria in sediments. Mar. Biol. 44: 293298.

Miller, D. J., M. B. Macek and B. D. Shur (1992). Complementarity between sperm surface $\beta$ - $(1,4)$-galactosyl-transferase and egg-coat ZP3 mediates sperm-egg binding. Nature 357: 589-593.

Miura, A., M. Horiguchi, D. Ogimoto and T. Matsumoto (1983). Nutritional interdependence among rumen bacteria during cellulose digestion in vitro. Appl. Environ. Microb. 45: 726-729.

Moers, M. E. C., M. Baas, J. W. De Leeuw and P. A. Schenck (1990). Analysis of neutral monosaccharides in marine sediments from the equatorial eastern Atlantic. Org. Geochem. 15(4): 367-373.

Mopper, K. (1977). Sugars and uronic acids in sediment and water from the Black Sea and North Sea with emphasis on analytical techniques. Mar. Chem. 5: 585-603.

Novitsky, J. A. (1986). Degradation of dead microbial biomass in a marine sediment. Appl. Environ. Microbiol. 52(3): 504-509.

Parsons, T. R., K. Stephens and J. D. H. Strickland (1961). On the chemical composition of eleven species of marine phytoplankton. J. Fish. Res. Bd. Canada 18(6): 1001-1016.

Pimenta, P. F. P., S. J. Turco, M. J. McConville, P. G. Lawyer, P. V. Perkins and D. L. Sacks (1992). Stage-specific adhesions of Leishmania promastigotes to the sandfly midgut. Science 256: 1812-1816.

Priest, F. G. (1992). Synthesis and secretion of extracellular enzymes in bacteria. Microbial Degradation of Natural Products. Weinham, VCH. 1-26.

Romankevich, E. A. (1984). Geochemistry of Organic Matter in the Ocean. Berlin, Springer-Verlag.

Sakugawa, H. and N. Handa (1985). Chemical studies on dissolved carbohydrates in the water samples collected from the North Pacific and Bering Sea. Oceanol. Acta 8(2): 185196.

Sakugawa, H., N. Handa and K. Yagi (1990). Distribution of glycosylglycerols and oligosaccharides in the marine environment and their ecological significance in the deep sea. Mar. Biol. 106: 309-313.

Schink, B. (1989). Mikrobielle Lebensgemeinschaften in Gewassersedimenten. Naturwiss. 76: 364-372.

Takahashi, M. and S. Ichimura (1971). Glucose uptake in ocean profiles with special reference to temperature. Mar. Biol. 11: 206-213.

Tanoue, E. and N. Handa (1987). Monosaccharide composition of marine particles and sediments from the Bering Sea and northern North Pacific. Oceanol. Acta 10(1): 91-99.

Taylor, C. D., S. O. Smith and R. B. Gagosian (1981). Use of microbial enrichments for the study of the anaerobic degradation of cholesterol. Geochim. Cosmochim. Acta 45: 
2161-2168.

Tissot, B. P. and D. H. Welte (1984). Petroleum Formation and Occurrence. Berlin, Springer Verlag.

Vaccaro, R. F. and H. W. Jannasch (1966). Studies on heterotrophic activity in seawater based on glucose assimilation. Limnol. Oceanogr. 11: 596-607.

van den Tweel, W. J. J., J. B. Kok and J. A. M. de Bont (1987). Reductive dechlorination of 2,4-dichlorobenzoate to 4-chlorobenzoate and hydrolytic dehalogenation of 4-chloro-, 4-bromo-, and 4-iodobenzoate by Alcaligenes denitrificans NTB-1. Appl. Env. Microbiol. 53(4): 810-815.

Walters, J. S. and J. I. Hedges (1988). Simultaneous determination of uronic acids and aldoses in plankton, plant tissues, and sediment by capillary gas chromatography of Nhexylaldonamide and alditol acetates. Anal. Chem. 60: 988-994.

Weimer, P. J. and J. G. Zeikus (1977). Fermentation of cellulose and cellobiose by Clostridium thermocellum in the absence and presence of Methanobacterium thermoautotrophicum. Appl. Environ. Microbiol. 33(2): 289-297.

Weis, W. I., K. Drickamer and W. A. Hendrickson (1992). Structure of a C-type mannose-binding protein complexed with an oligosaccharide. Nature 360: 127-134.

Weiss, M. S., U. Abele, J. Weckesser, W. Welte, E. Schiltz and G. E. Schulz (1991). Molecular architecture and electrostatic properties of a bacterial porin. 254: 1627-1630.

Westrich, J. T. and R. A. Berner (1984). The role of sedimentary organic matter in bacterial sulfate reduction: the G model tested. Limnol. Oceanogr. 29(2): 236-249.

Whelan, J. K. and K.-C. Emeis (1992). Sedimentation and preservation of amino compounds and carbohydrates in marine sediments. Organic Matter: Productivity, Accumulation, and Preservation in Recent and Ancient Sediments. New York, Columbia University Press. 176-200.

Widdel, F. (1988). Microbiology and ecology of sulfate- and sulfur-reducing bacteria. Biology of Anaerobic Microorganisms. New York, Wiley. 469-585.

Yamaoka, Y. (1983). Carbohydrates in humic and fulvic acids from Hiroshima Bay sediements. Mar. Chem. 13: 227-237. 


\section{Chapter Two: Nuclear Magnetic Resonance Spectroscopy of Carbohydrates}

\section{Introduction}

Nuclear magnetic resonance spectroscopy (NMR) is a sensitive, non-destructive technique for determination of chemical structure and conformation. Progress in NMR spectroscopy within the last decade has led to enormous advances in the complexity of chemical structures which can be resolved. While development of high field magnets, rapid expansion of multiphase, multi-dimensional techniques, and improvements in computer hardware and software have reduced the difficulty of analyzing complex structures and spectra, properly setting up and interpreting an NMR experiment still requires some background and experience. This chapter will provide a general overview of one- and twodimensional NMR spectroscopy, a very brief discussion of the application of NMR techniques to carbohydrate analysis, and finally more detailed discussion of specific techniques as used in a survey of carbohydrate standards, and as applied in Chapter 3 to a survey of phytoplankton carbohydrates.

Nuclear magnetic resonance spectroscopy has been applied extensively in chemical and biochemical research, including detailed structural characterization of industrially and biomedically important bacterial polysaccharides (Backman-Marklund et al., 1990; Cassels et al., 1990; Doco et al., 1990; Fontaine et al., 1991, for example). Far less work has been done on carbohydrates of geochemical interest. A search of the literature revealed only a handful of reports which include NMR characterization of marine carbohydrates, including two studies which utilized ${ }^{13} \mathrm{C}$ - and ${ }^{1} \mathrm{H}-\mathrm{NMR}$ to characterize hydrolysis products of red algal polysaccharides (Jaseja et al., 1989; Geresh et al., 1990). The linkage points of the reserve glucan of the prymnesiophyte Emiliania huxleyi have been investigated using ${ }^{13} \mathrm{C}$ NMR (Varum et al., 1986), and the structure of sulfated galactans isolated from the macrophyte Furcellaria lumbricalis was studied using both ${ }^{13} \mathrm{C}$ - and ${ }^{1} \mathrm{H}-\mathrm{NMR}$ (Knutsen $e t$ al., 1990). Carbon-13 NMR has also been used for gross characterization of humic materials isolated from groundwater (Buddrus et al., 1989; Lambert et al., 1992) and soils(Wershaw et al., 1990). Solid-state ${ }^{13} \mathrm{C}-\mathrm{NMR}$ has been used to characterize green algae (Berkaloff et al., 1983; Zelibor et al., 1988; Saito et al., 1991) and decaying mangrove leaves (Benner et al., 1990).

Solid-state ${ }^{13} \mathrm{C}-\mathrm{NMR}$ was also used recently in an investigation of dissolved organic matter from the North Pacific. A comparison of samples collected at the surface, oxygen minimum zone $(765 \mathrm{~m})$, and a depth of $4000 \mathrm{~m}$ showed changes in the gross 
composition of dissolved organic matter $>1000$ daltons. A sharp decrease in the resonances attributed to carbohydrate carbon was particularly prominent (Benner et al., 1992). While solid-state ${ }^{13} \mathrm{C}-\mathrm{NMR}$ provides valuable information about the general types of carbon present in a sample, and is the only type of NMR which can be done on insoluble samples, the line-widths in solid-state ${ }^{13} \mathrm{C}$-NMR tend to be broad, and resolution is generally poor compared to solution NMR. Solid-state NMR also lacks the vast range of multidimensional and heteronuclear experiments which can be used to unravel the complexities of a structure in solution. In addition, as discussed below, the conformation of closely related structures in solution can differ greatly; the solid-state ${ }^{13} \mathrm{C}-\mathrm{NMR}$ of such structures would be virtually indistinguishable, while the solution ${ }^{13} \mathrm{C}$ - and ${ }^{1} \mathrm{H}-\mathrm{NMR}$ spectra would clearly highlight such differences. As mentioned in Chapter 1, these differences in solution conformation help determine the biological, physical, and chemical properties of a structure, including its relative susceptibility to biological uptake, photochemical degradation, and enzymatic attack, for example.

\section{Experimental conditions}

The spectra were all acquired on a Brucker AC $3000300 \mathrm{MHz}$ spectrometer. All of the mono-, di-, and oligosaccharides standards were used as purchased from Aldrich Chemical Co. and Sigma Chemicals; saturated $\mathrm{D}_{2} \mathrm{O}$ solutions of the polysaccharides (U.S. Biochemicals) were filtered ( $0.2 \mu$ filter) prior to use to remove insoluble material which would have affected spectral quality. Sample quantities varied according to the type of experiment and the solubility of the material. Typically, $5-6 \mathrm{mg}$ of a mono-, di-, or trisaccharide were used for high quality 1- and 2-dimensional (1-D and 2-D) proton spectra; much smaller sample quantities (on the order of 10s-100s of $\mu \mathrm{g}$ ) would have been sufficient, although acquisition of the spectra would have required more instrument time. Due to the low natural abundance of ${ }^{13} \mathrm{C}$ and its inherently lower NMR sensitivity, much more material is required for carbon experiments. One hundred $\mathrm{mg}$ were used to collect the 1-D ${ }^{13} \mathrm{C}$ and heteronuclear 2-D spectra of isomaltose; again, less material would have required more aquisition time. Solublility is a limiting factor for oligo- and polysaccharides, as explained below, but approximately $50 \mathrm{mg}$ of pullulan, a linear glucose polysaccharide of 200,000 daltons, were more than sufficient for high-quality 1-D ${ }^{13} \mathrm{C}$ and heteronuclear 2-D spectra. All samples were dissolved in 100.0 atom\% D D 20 (Aldrich). All chemical shifts are reported using the standard " $\delta$ " notation, in which the reported shift (units of p.p.m.) is relative to the chemical shift of a standard. Unless otherwise noted, the proton spectra were referenced to the solvent HOD peak ( $\delta=4.8$ or 4.76 , as noted). Carbon spectra 
were referenced to internal acetonitrile $(\delta=117.2)$. Most of the oligo- and polysaccharides, as well as all of the phytoplankton spectra discussed in Chapter 3, were collected with the sample probe at a temperature of $298 \mathrm{~K}$.

\section{One- and two-dimensional NMR}

General explanations of NMR spectroscopy can be found in organic chemistry textbooks; however, the field has advanced rapidly, and most books and papers dealing with NMR which are more than 5-7 years old are incomplete, and do not cover many techniques now commonly used. Modern NMR Techniques for Chemistry Research (Derome, 1987) is an excellent general introduction to 1- and 2-dimensional methods; Basic One- and Two-

Dimensional NMR Spectroscopy (Friebolin, 1991) provides explanations about a variety of experiments as well as information on the interpretation of spectra in terms of chemical structures, and Two-Dimensional NMR Methods for Establishing Molecular Connectivity (Martin and Zektzer, 1988) has more specialized discussion of specific two dimensional techniques and composite pulse sequences.

In brief, in a liquid sample the net magnetic moments of spin-1/2 nuclei such as ${ }^{1} \mathrm{H}$ and ${ }^{13} \mathrm{C}$ will align either with or against an external magnetic field $\mathbf{B}$. At equilibrium, slightly more nuclei will take the lower energy $\alpha$-orientation (with the external magnetic field) as compared to the $\beta$ orientation (against the magnetic field), in accordance with a Boltzmann distribution (Fig. 2.1). In an NMR experiment, the net magnetization of the nuclei in a sample is redistributed through the application of a radiofrequency pulse, i.e., the nuclei in the lower energy $\alpha$-orientation acquire sufficient energy to bring them to the $\beta$ orientation, and the time-course of their return to equilibrium is observed. The rate and means by which this process occurs is directly dependent upon the local chemical environment of each nucleus in a sample. In terms of vector representations (Fig. 2.2), application of a radiofrequency pulse to the sample tips the net magnetization vector from its equilibrium position along the $\mathrm{z}$ axis (i.e., in the direction of the external magnetic field B). The NMR receiver detects the signal generated in the $x-y$ plane. Through transverse and longitudinal relaxation processes, the net magnetization gradually returns to its equilibrium orientation along the $\mathrm{z}$-axis. The decrease in the vector components in the $\mathrm{x}-\mathrm{y}$ plane is detected by the NMR receiver, and is referred to as the free induction decay (FID). The FID, a function of time, is Fourier transformed to yield peaks as a function of frequency-the familiar NMR spectrum. 


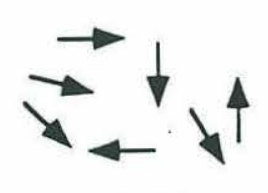

nuclei in a

liquid sample

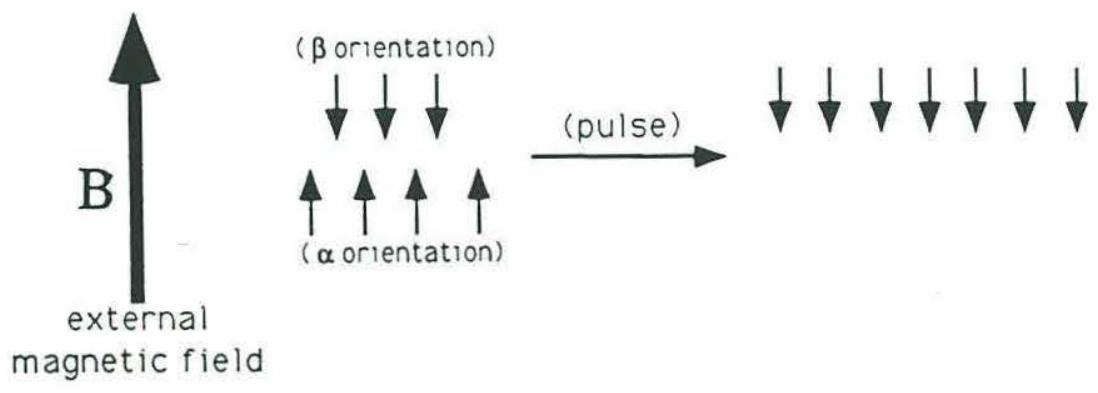

Fig. 2.1

In an external magnetic field (B), a slight majority of spin-1/2 nuclei will take the lowerenergy " $\alpha$ " orientation in the direction of the magnetic field. A radiofrequency pulse supplies the energy necessary to convert these spins to the higher energy " $\beta$ " orientation.

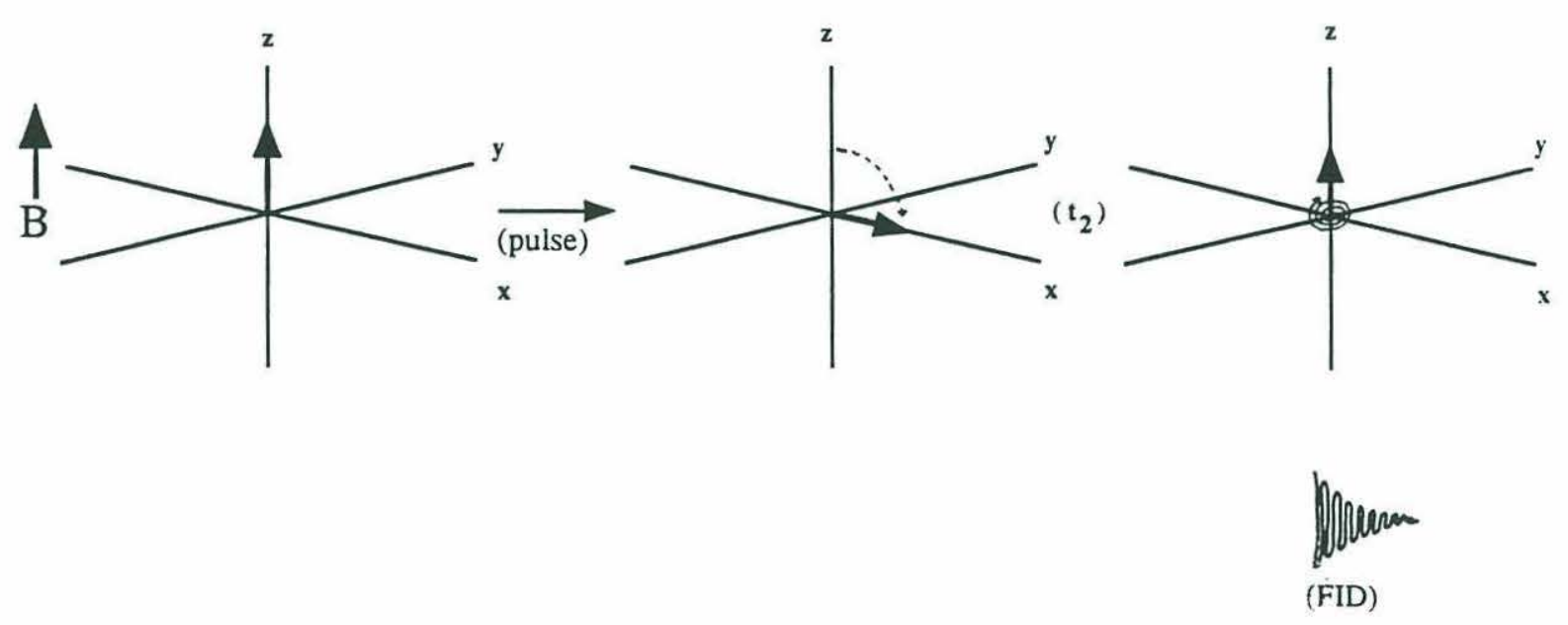

Fig. 2.2

Effect of a radiofrequency pulse on the net magnetization vector 
An NMR experiment can also be represented by the following sequence:

$$
\text { preparation period }- \text { pulse }-\mathrm{t}_{2} \text { (FID) }
$$

Since the data collected during an NMR experiment is the sum of a large number of repeated observations, a 'preparation period' is required to allow the sample to come to equilibrium after application of the previous pulse, so that the starting conditions for each observation are equivalent. The sample is pulsed, and during time $t_{2}$ the receiver is switched on to observe the nuclei (observe the free induction decay).

Two-dimensional NMR techniques, which have become widely used in the past decade, provide a means of determining a significant amount of information about even complex structures. Fundamentally all $2 \mathrm{D}$ techniques can be described in general terms with the following modifications to the sequence explained above:

$$
\text { preparation period }- \text { pulse }-t_{1}-\text { pulse }-t_{2}(F I D)
$$

In terms of vector diagrams (Fig. 2.3), the first pulse tips the net magnetization vector from its equilibrium position along the $\mathrm{z}$ axis; a $90^{\circ}$ pulse along the $+\mathrm{y}$ axis, for example, tips the vector to the $+x$ axis. The time interval $t_{1}$ is the evolution time, an incremented delay period during which the magnetiztion vector precesses in the $\mathrm{x}-\mathrm{y}$ plane.The net magnetization vector can be trigonometrically decomposed to its components along the $\mathrm{x}$ and $\mathrm{y}$ axes. After interval $t_{1}$, a second $90^{\circ}$ pulse (+y in this case) is applied. The second pulse in effect tips the $\mathrm{x}$ component to the $-\mathrm{z}$ axis, where it is not observed by the NMR receiver. The $\mathrm{y}$ component is unaffected by the second pulse, and therefore generates a signal in the receiver. By incrementing $t_{1}$ by a fixed amount throughout the experiment, a succession of signals is obtained as a function of time. The $2 \mathrm{D}$ experiment is therefore built up as a function of the time increment $t_{1}$, which is changed by a fixed amount for each 'step' in the experiment, and by $t_{2}$, during which the FID is observed. Fourier transform yields the 2D matrix as a function of frequencies $F_{1}$ and $F_{2}$, the transformations of $t_{1}$ and $t_{2}$. Variations in pulse angles, intervals, and phases differentiate two-dimensional experiments from one another. Three- and four-dimensional NMR experiments are performed by adding further incremented time intervals (analogous to $t_{1}$ ) and pulse sequences; such experiments have already been applied to the characterization of large proteins in solution (Clore and Gronenborn, 1991). 

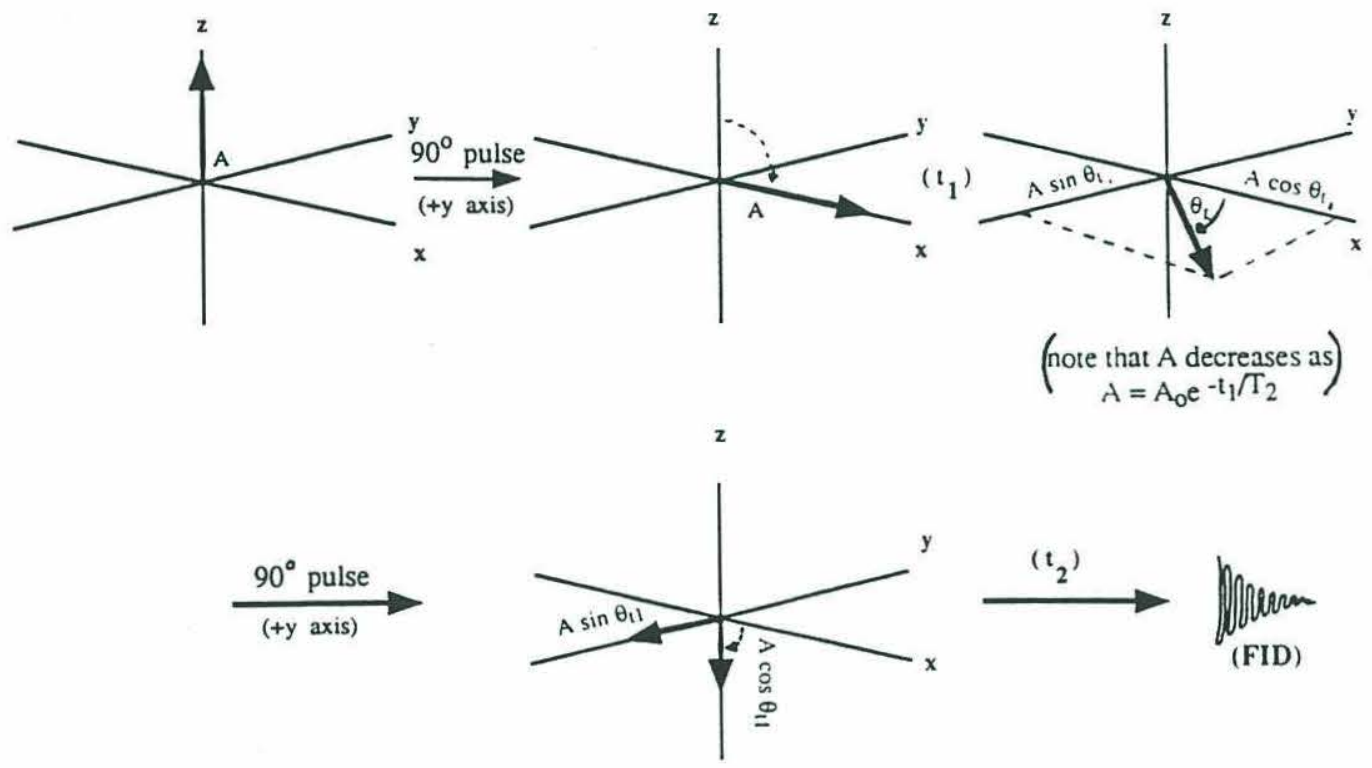

Fig. 2.3

Vector-model of a two-dimensional NMR experiment. Application of the first $90^{\circ}$ pulse along the $+y$ axis brings the net magnetization vector to the $+x$ axis. After an incremented time $t_{1}$, application of a second 90 pulse tips the $+x$ component of the vector to the $-z$ axis; only the precession of the component remaining in the $x-y$ plane is detected by the receiver. 


\section{Application of NMR to Studies of Carbohydrates}

NMR spectroscopy is particularly useful for carbohydrate analysis, since many structural features, such as anomeric linkage and linkage position, are difficult or impossible to resolve with standard carbohydrate analytical techniques. In addition, an NMR spectrum reflects the solution conformation of a structure, which can provide important information about biochemical fit and function of carbohydrate-protein complexes (Weis et al., 1992), for example.

The chemical shifts of nuclei such as ${ }^{1} \mathrm{H}$ and ${ }^{13} \mathrm{C}$ are determined by the immediate magnetic environment of the nucleus. Seemingly equivalent protons, for example the - $\mathrm{CH}_{2}$ protons of the \#6 carbon in glucose (Fig. 2.4), have different chemical shifts. In addition, the presence of neighboring protons results in splitting of proton resonances into multiplet peaks---one neighbor splits a resonanace into a doublet, two equivalent neighbors yield a triplet, three a quartet. Non-equivalent neighbors produce even more complex splitting patterns. For glucose, for example, the two \#6 protons have different chemical shifts and different splitting patterns ("J couplings"), although each is coupled only to the other and to the \#5 proton.

For one-dimensional proton spectra of carbohydrates, a few characteristic features are readily apparent. The well-resolved doublets in the region $\delta=4.4-5.6$ are due to anomeric protons, which resonate far downfield because of their proximity to two oxygens - the ring oxygen, and the anomeric hydroxyl group. As a general rule, $\alpha$ anomeric protons resonate downfield of $\beta$ protons (due to their orientation relative to the ring oxygen), and $\beta$ protons typically have coupling constants $\mathrm{J}=\sim 8 \mathrm{~Hz}$ as compared to $\alpha$ $\mathrm{J}$-values of 2-3 Hz. Since J-coupling of the anomeric proton is due to coupling with the \#2 proton, the only exception is for carbohydrates such as galactose, which have the \#2 proton in the axial position.

One of the difficulties of carbohydrate analysis is that two to four different forms of a free reducing sugar are typically present in solution at thermal equilibrium (Table 2.1). In an NMR spectrum, the region $\delta=3.1-4.0$ is complex even when just a single form of monosaccharide is present, as in Fig. 2.4, which shows a solution of $\beta$-glucose (the spectrum was recorded before the conversion to an equilibrium mixture of $\alpha$ - and $\beta$-forms took place.) The \#6 protons resonate in the region $\delta=3.67-3.75$ and $\delta=3.86-3.93$, while the $\# 2$ proton is found upfield at $\delta=3.2-3.8$. The \#3, \#4, and \#5 proton resonances overlap in the region $\delta=3.35-3.52$. Once equilibrium between $\alpha$ and $\beta$ forms has been reached (Fig. 2.5), the spectrum becomes even more complex. 


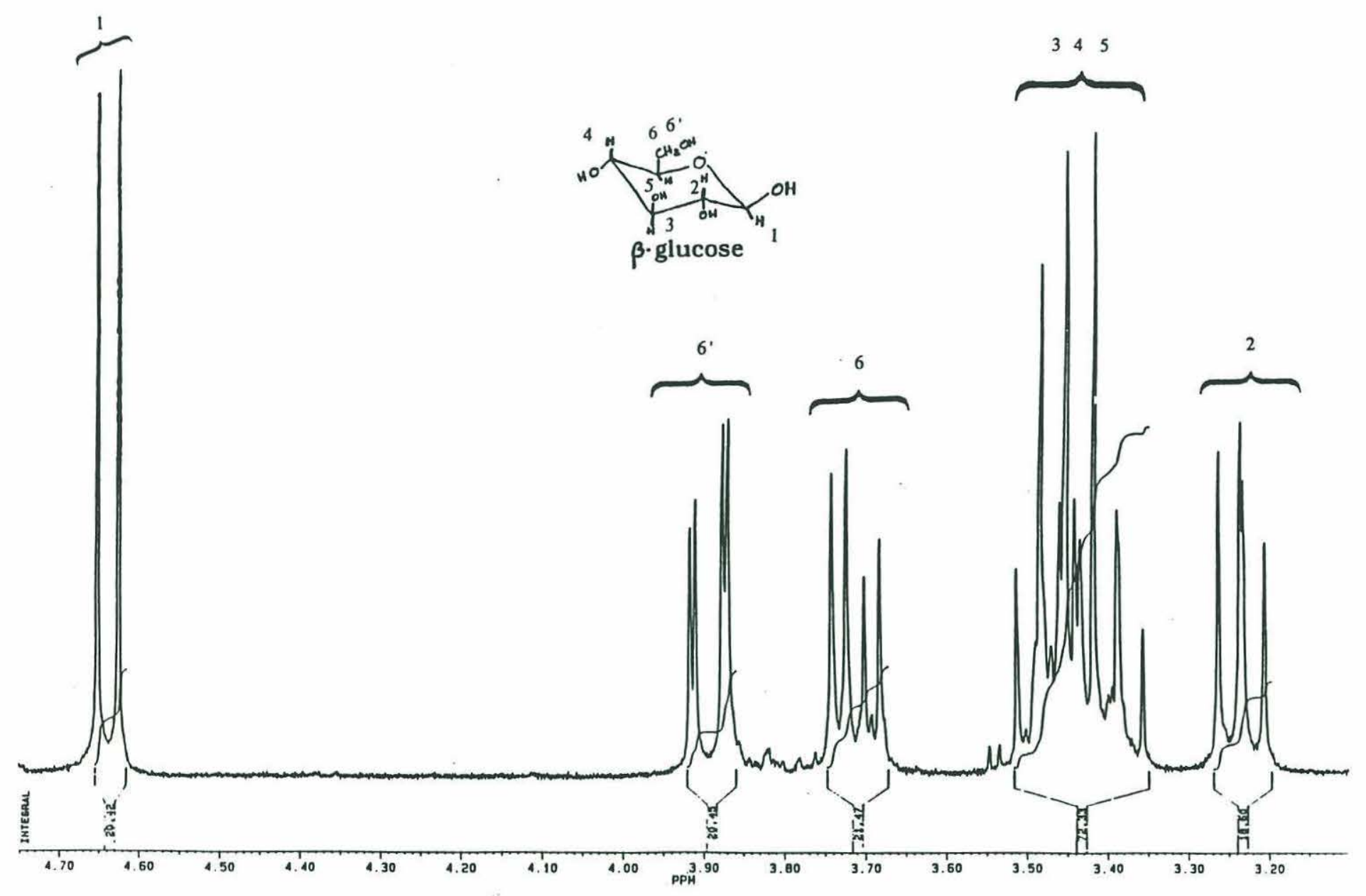

Fig. 2.4

${ }^{1} \mathrm{H}$ spectrum of $\beta$-glucose in solution. (HOD resonance set to $\delta=4.8$ ). 
Composition of reducing sugars in $\mathrm{D}_{2} \mathrm{O}$ at $31^{\circ}{ }^{\circ}$ **

(\% distribution among forms)

$\alpha$ pyranose $B \quad \alpha$ furanose $B$

D-glucose

38

62

D-galactose

30

64

2.5

3.5

D-mannose

65.5

34.5

0.6

0.3

D-arabinose

60

35.5

2.5

2

D-ribose

21.5

58.5

6.5

13.5

D-xylose

36.5

63

$<1$

D-rhamnose

60

40

D-fucose

28

67

5

Table 2.1

The equilibrium distribution of reducing sugars in solution.

(** table adapted from Modern Carbohydrate Chemistry, R.W. Binkley. New York, Dekker, 1988, 343pp.) 


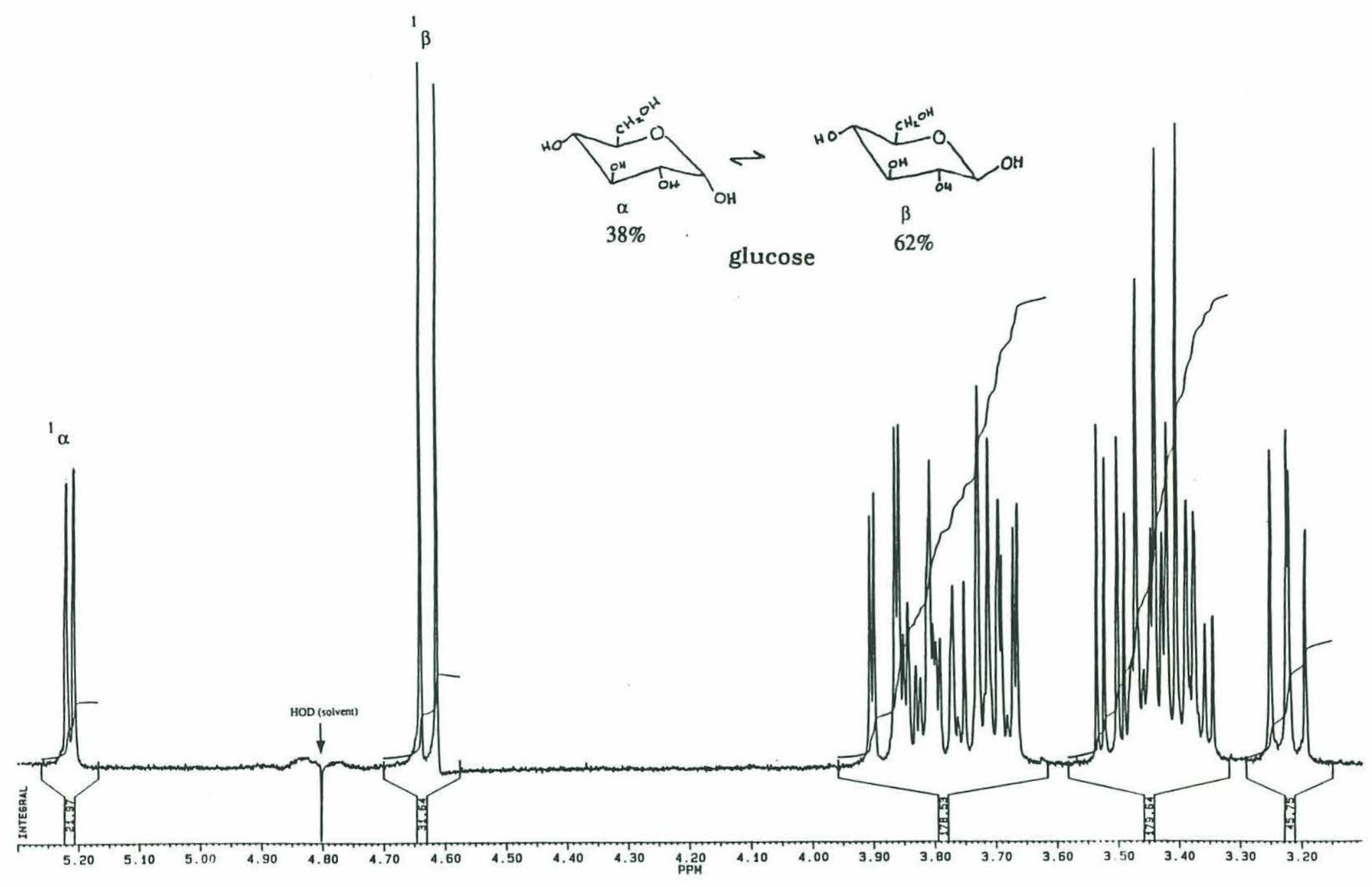

Fig. 2.5

${ }^{1} \mathrm{H}$ spectrum of an equilibrium mixture of $\alpha$ - and $\beta$-glucose in solution. (HOD resonance set to $\delta=4.8$ ). 
For larger oligo- and polysaccharides, the 1-D spectrum cannot be resolved; 2-D techniques are required in order to assign resonances to specific protons.

\section{Survey of carbohydrate standards}

A library of carbohydrate standard spectra was collected in order to directly evaluate the spectral differences among the neutral monosaccharides most commonly found in marine samples, and to determine the differences between oligo-and polysaccharides of similar composition. While extensive tables of mono- (Bock and Pederson, 1983), oligo- (Bock et al., 1984), and polysaccharide (Gorin, 1981) ${ }^{13} \mathrm{C}$ chemical shifts have been published, less data is available for proton NMR shifts. Additionally, as discussed below for the case of isomaltose, some of the data in the published tables was collected over 20 years ago on low field instruments, and can be improved upon with the help of high field instruments. A series of studies on the conformation and proton shifts of a set of O-methyl di- and trisaccharides have been published (Backman et al., 1988; Baumann et al., 1988; Jansson et al., 1988; Baumann et al., 1989; Backman et al., 1990), but they do not provide a complete survey of the neutral carbohydrates of interest in this work. Most of the tables record the shifts for O-methyl derivatives of mono- and disaccharides, whose chemical shifts differ significantly (particularly for anomeric and \#2 protons) from those of the free reducing sugars. In addition, most of the spectra were recorded at a temperature of $70^{\circ} \mathrm{C}$, which also affects chemical shifts.

Tables of proton and carbon chemical shifts are available for the monosaccharides glucose, galactose, mannose, allose, gulose, xylose, arabinose, ribose, and lyxose (Bock and Thogersen, ), and a few oligosaccharides including maltose and maltotriose (Morris and Hall, 1982), cellotriose (Ikura and Hikichi, 1987), kojibiose, nigerose, sophorse, and laminaribiose (DeBruyn, 1991). Chemical shifts have also been reported for the glucan polysaccharides dextran (Morris and Hall, 1982), pullulan , and inulin (McIntyre and Vogel, 1991). Other than these examples, however, few chemical shift tables of free reducing neutral carbohydrates, and even fewer two-dimensional spectra, have been reported. The quality of some published spectra is poor due to high viscosity of the sample (McIntyre et al., 1990; McIntyre and Vogel, 1990; McIntyre and Vogel, 1991). In order to have a uniform reference base, the spectra of a range of mono-, oligo-, and polysaccharides were collected, several of which are discussed below.

All of the proton spectra were collected using solvent suppression (standard Brucker software, PRESAT.AUR, for one-dimensional proton spectra). The twodimensional COSY (explained further below) were collected using the COSYHG.AUR 
Brucker program, which also included solvent suppression. Typically 16-32 scans (in a few cases, 64 scans) were collected per $t_{1}$ increment. In order to decrease crowding about the diagonal, a pulse width of $45^{\circ}$ was used for the COSY experiments (Derome, 1987). Carbon- 13 spectra were collected in the proton-decoupled mode; a $60^{\circ}$ pulse (calibrated as $3.6 \mu \mathrm{sec}$ ) was found to be most effective. DEPT experiments were collected with the Brucker program DEPT.AUR. The heteronuclear two-dimensional proton-carbon spectra (HETCOR experiments) were collected using XHCORR.AUR. Details of acquisition and processing parameters for ${ }^{13} \mathrm{C}$ and 2D NMR spectra are given in Appendix A.

\section{Oligosaccharides}

\section{$1 D$ Spectra}

The 1-D spectra of gentiobiose (glucose- $\beta(1,6)$-glucose) and isomaltose (glucose$\alpha(1,6)$-glucose) (Fig. 2.6) illustrate a dramatic difference in solution conformation, reflected by their NMR spectra, which is solely due to anomeric linkage. (Since these samples were referenced only to the HOD peak, set to $\delta=4.8$, the chemical shift values are relative.) The reducing end of the disaccharide is referred to as ring I; the non-reducing monomer is referred to as ring II in the following discussion. The main common features of the gentiobiose and isomaltose spectra are the free anomeric ends of ring I at $\delta=5.21$ and 4.64. The $\alpha$ anomeric linkage (ring II) for isomaltose is at $\delta=4.91$, while the $\beta$ anomeric linkage for gentiobiose is at $\delta=4.50$. The effect which distant protons have on chemical shift is illustrated by the small, slightly-offset doublet at $\delta=4.49$ for gentiobiose, which represents ring II bound to ring I with an $\alpha$ free anomeric end. Similarly, the broad and jagged appearance of the $\alpha$ anomeric linkage of isomaltose is due to the slightly differing chemical shifts of ring II bound to ring I with a free $\alpha$ end, as compared to ring II bound to ring I with a free $\beta$ end. Although gentiobiose and isomaltose differ only in the orientation of the $(1,6)$ bond, most of their proton chemical shifts are quite different, even for protons distant from the bond. The region $\delta=3.2-4.0$ contains the \#2-\#5 proton resonances for each disaccharide; significant differences between the two disaccharides are apparent. Deconvolution of the overlapping peaks requires 2-D techniques.

\section{Correlation Spectroscopy (COSY)}

Figure 2.7, the 2-D ${ }^{1} \mathrm{H}$ COSY of gentiobiose, provides an example of a common twodimensional technique, a COSY (COrrelation SpectroscopY) showing the relation of (in this case) protons in a structure. Gentiobiose proton shifts have not been previously 


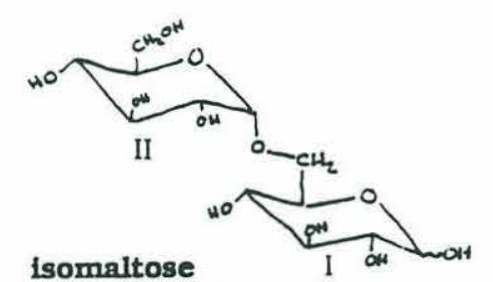

glucose- $\alpha(1,6)$-glucose
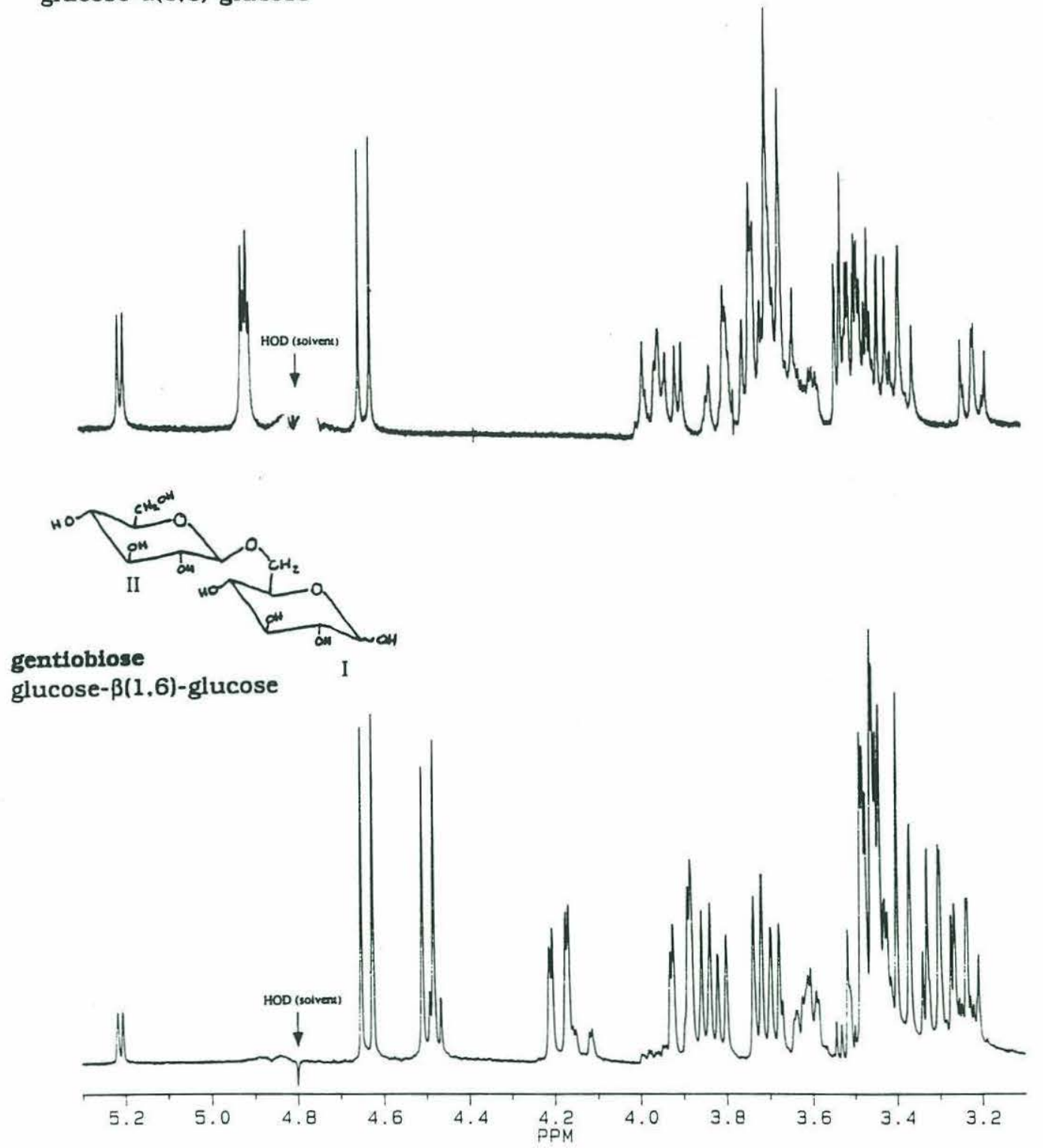

Fig. 2.6

${ }^{1} \mathrm{H}$ spectra of isomaltose and gentiobiose. (HOD resonance set to $\delta=4.8$ ). 


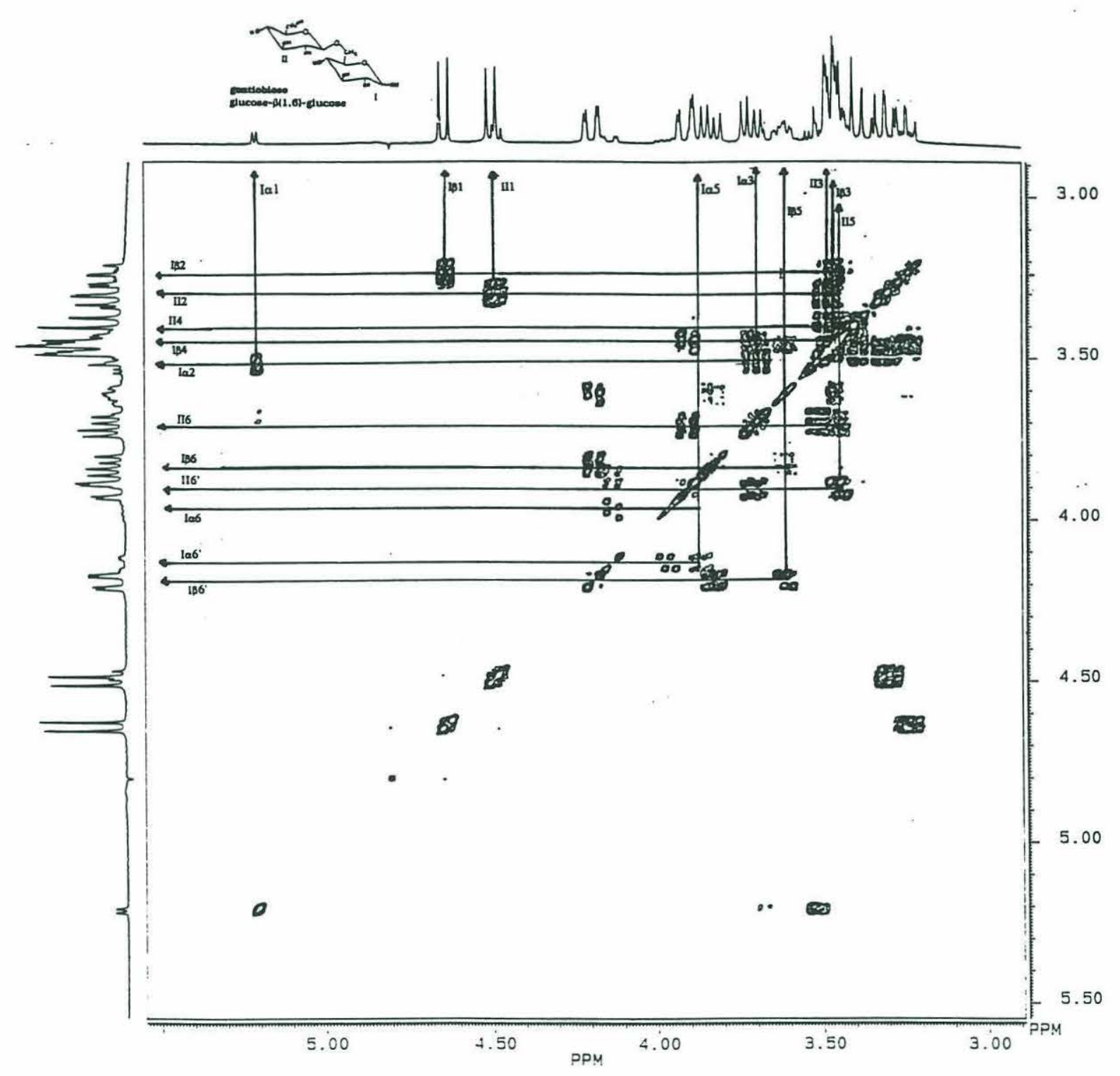

Fig. 2.7

Gentiobiose ${ }^{1} \mathrm{H} \operatorname{COSY} 45^{\circ}$. (HOD resonance set to $\delta=4.8$ ). 
reported in the literature. Although a range of experiments is usually necessary to completely assign a structure, the entire gentiobiose structure was assigned on the basis of this well-resolved COSY (Table 2.2). The two one-dimensional spectra along the two axes are a guide for reading the plot; the results of the actual experiment are within the matrix. (The horizontal axis is referred to as the "F2" axis, while the vertical axis is the "F1" axis.) Since this is a homonuclear (proton-proton) experiment, the matrix is symmetrical about the diagonal, and only one half of the matrix is actually needed in order to extract useful information.

During the second pulse of a 2D ${ }^{1} \mathrm{H}$ COSY experiment, magnetization is transferred from protons to their directly connected neighbors. The diagonal peaks in the matrix result from non-transferred magnetization during the second pulse, so any proton which has no directly connected neighbors will show only a diagonal peak. A cross-peak off the diagonal indicates that a proton has a directly connected neighbor. For example, an anomeric proton has only one nearest neighbor, the \#2 proton. The \#2 proton has both the anomeric proton and the \#3 proton as neighbors. As shown in Fig. 2.7, the anomeric proton from ring I has only one crosspeak below it, which must therefore originate from its interaction with the \#2 proton. Tracing down the peak from the $\# 2$ proton shows an additional crosspeak, which must originate from the \#3 proton. In this fashion, the connectivities around an entire ring can be traced, as shown in Fig. 2.7.

The 2D COSY of isomaltose (Fig. 2.8) helps unravel the complexities of the onedimensional proton spectrum in a similar fashion, although the entire structure cannot be traced as easily through the matrix as for gentiobiose. A cursory comparison with Fig. 2.7 reveals that the \#6 protons are especially affected by anomeric configuration of the linkage. In gentiobiose, the \#6 protons in both rings yield a well-resolved doublet of doublets (at $\delta=4.3$ for ring I and $\delta=3.9$ for ring II) and an asymmetric quartet at $\delta=3.85$ for ring I and 3.7 for ring II. The \#6 protons in isomaltose are less well-resolved and therefore harder to distinguish in the matrix. The anomeric protons, of course, are well resolved, so the crosspeak leading to the $\# 2$ protons is also easily found. Expanding the region between $\delta=$ 3.1 and 4.0 (Fig. 2.9) clarifies crosspeaks corresponding to the \#2, \#3, and ring I \#4 protons, but the matrix is quite complex. Several other NMR spectra can aid in assignment of the resonances corresponding to the other isomaltose protons and carbons.

\section{Carbon-13}

A proton-decoupled carbon spectrum of isomaltose (Fig. 2.10) shows the resonances corresponding to the carbons in the disaccharide. As with protons, carbon nuclei resonate 
chemical shift $\quad$ assignment (ring number, proton number)

$\begin{array}{ll}3.24 & \text { I } \beta 2 \\ 3.30 & \text { II2 } \\ 3.40 & \text { II } \\ 3.45\{3.61\} & \text { I } \beta 4 \\ 3.45\{3.70\} & \text { II5 } \\ 3.46 & \text { I } \beta 3 \\ 3.48 & \text { II } 3 \\ 3.52 & \text { I } \alpha 2 \\ 3.61 & \text { I } 55 \\ 3.70 & \text { I } \alpha 3 \\ 3.71 & \text { II6 } \\ 3.85 & \text { I } \beta 6 \\ 3.87 & \text { I } \alpha 5 \\ 3.90 & \text { II } 6^{\prime} \\ 3.97 & \text { I } \alpha 6 \\ 4.13 & \text { I } \alpha 6^{\prime} \\ 4.19 & \text { I } 66^{\prime} \\ 4.50 & \text { I1 } \\ 4.64 & \text { I } 1 \\ 5.22 & \text { I } \alpha 1\end{array}$

not determined: $\quad \mathrm{I} \alpha 4$

\section{Table 2.2}

${ }^{1} \mathrm{H}$-NMR chemical shifts of gentiobiose. The shifts are referenced to the internal HOD peak, which was set to $\delta=4.8$. Note that protons with the same chemical shifts (IB4 and II5) are resolved within the 2D COSY. The chemical shift given in \{\} brackets is the 'cross reference' line for the 2D COSY in Fig. 2.7. 


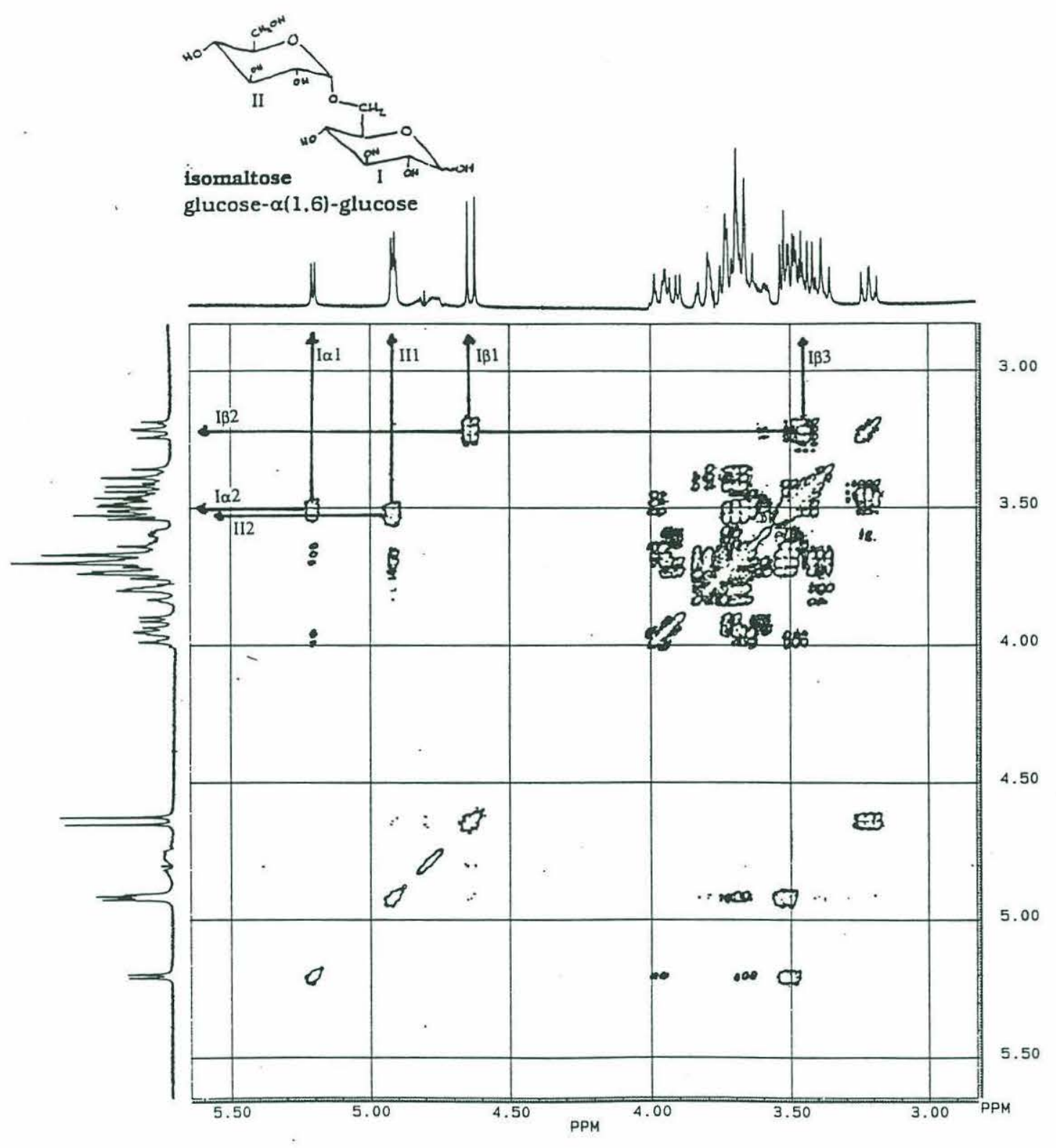

Fig. 2.8

Isomaltose ${ }^{1} \mathrm{H} \operatorname{COSY} 45^{\circ}$ (HOD resonance set to $\delta=4.8$ ). 


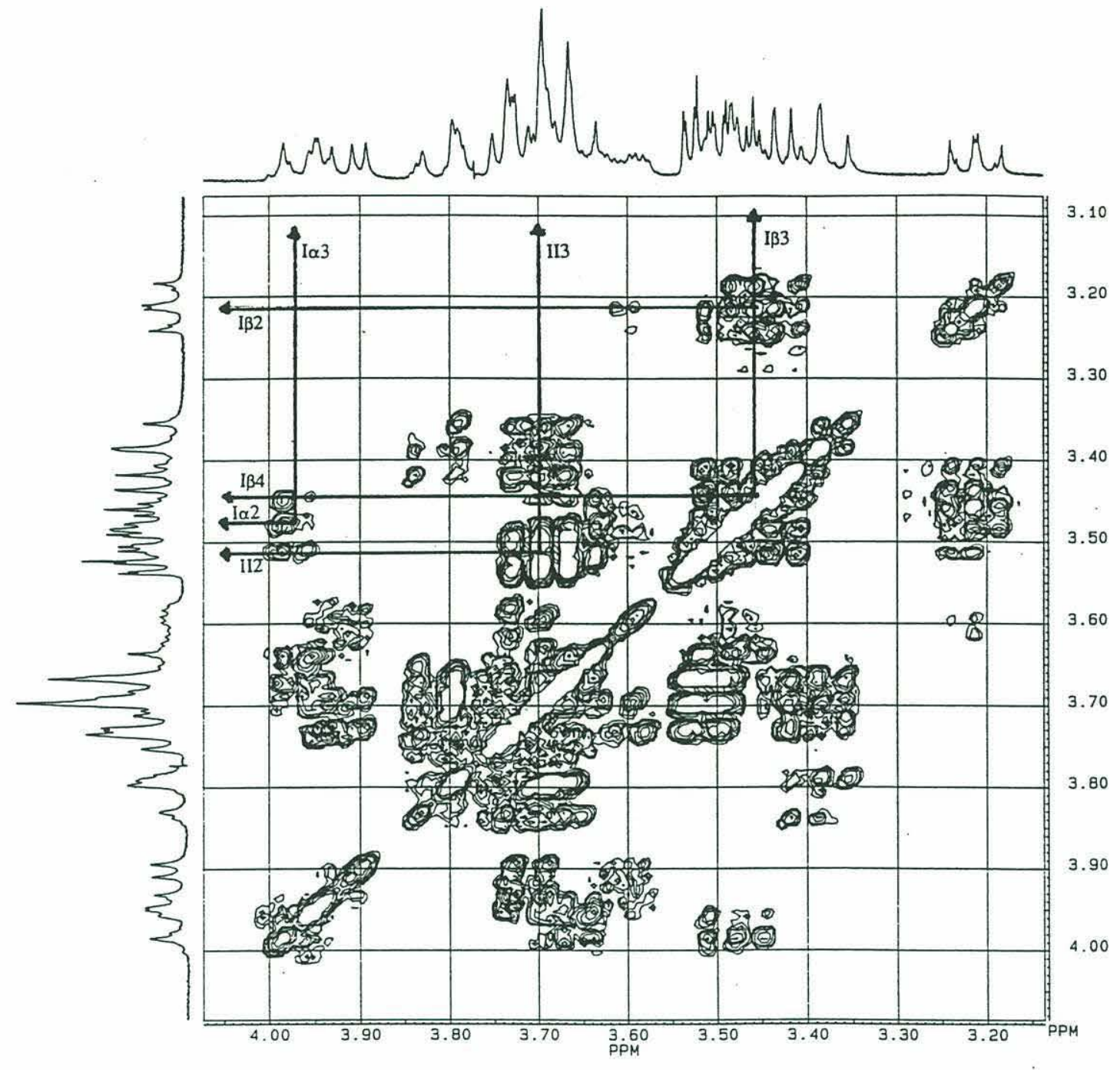

Fig. 2.9

Expansion of isomaltose ${ }^{1} \mathrm{H}$ COSY 45 . 


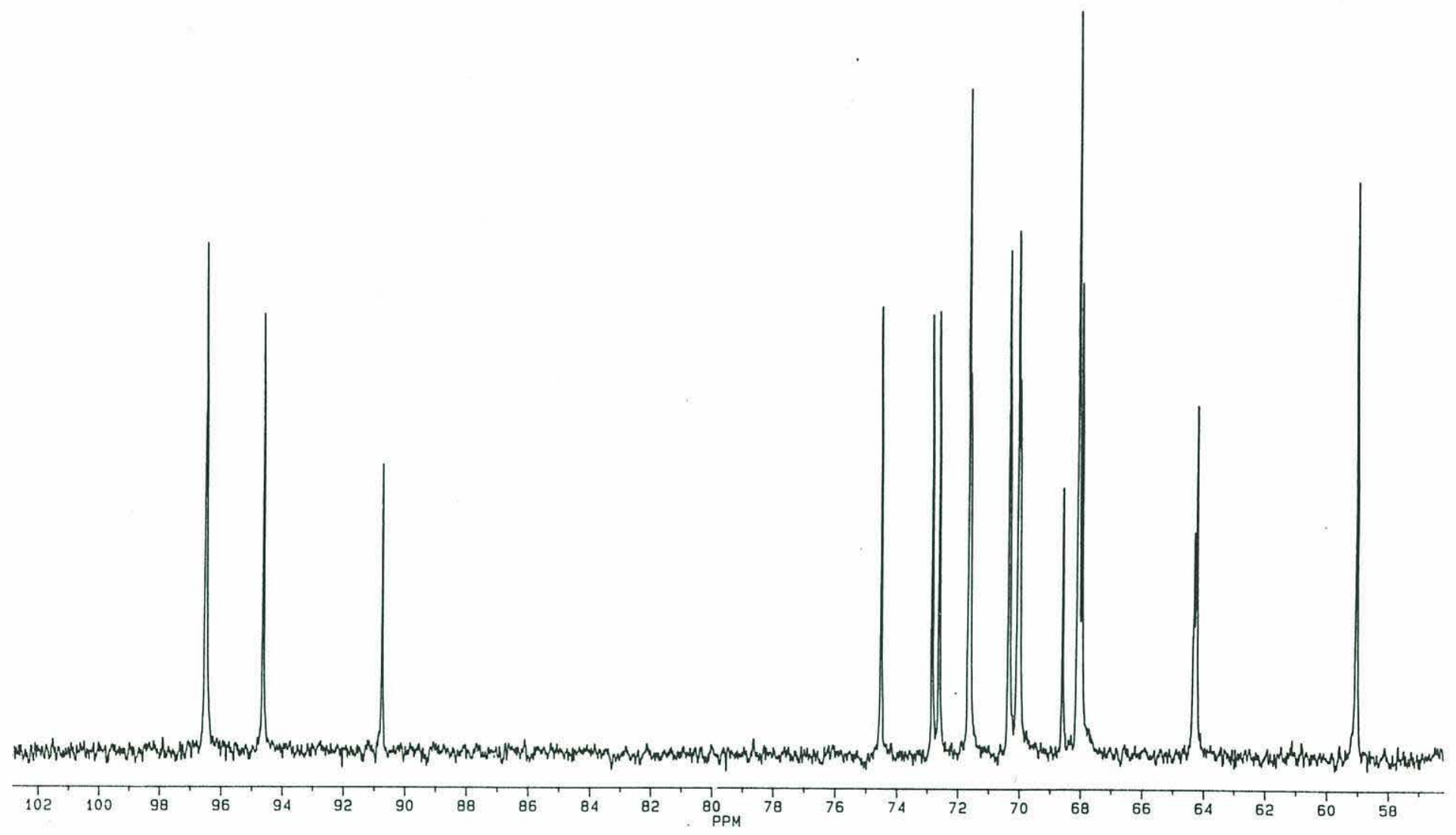

Fig. 2.10

${ }^{13} \mathrm{C}$ spectrum of isomaltose; pulse width of $60^{\circ}$. Temperature $=298 \mathrm{~K}$, internal standard: acetonitrile $(\delta=117.2)$. 
at positions in a spectrum which reflect their local magnetic environments. Carbons in anomeric positions typically resonate downfield near $\delta=100$, while the \#6 carbons are often found furthest upfield in the region near $\delta=60$. The positions of these carbon nuclei were determined with reference to internal acetonitrile $(\delta=117.2)$. Seventeen distinct resonances were detected (Table 2.3). Since the COSY spectrum shows that the two anomeric forms ( $\alpha$ and $\beta$ ) of ring I of isomaltose are prominent in solution, eighteen carbon resonances (six each from the $\alpha$ and $\beta$ forms of ring I, six from ring II) might have been found in the ${ }^{13} \mathrm{C}$ spectrum. A comparison of peak areas and the near-overlap of several of the resonances in the region $\delta=69-73$ indicate that two carbons have the same chemical shift.

Both chemical shifts and relative peak intensities are listed in Table 2.3. (Note that the relative intensities are only a general guide to the abundance of a particular carbon in a sample, since "intensity" is actually peak height, and the width of the lines in the spectrum varies.) In addition, carbon intensities can be enhanced due to the nuclear Overhauser effect, or they can be decreased due to incomplete relaxation between pulses. Since a pulsewidth of $60^{\circ}$ was selected, full carbon intensity may not have been recorded. The intensities in Table 2.3 can be grouped into broad catagories: six carbons with intensities $>0.65$, which belong to the II ring, six with relative intensities between 0.47 and 0.61 (the ring I $\beta$ carbons), and five with intensities between 0.28 and 0.51 (the ring I $\alpha$ carbons). One of the $\alpha$ carbons must overlap with another carbon, and the likely candidate is the ring II \#4 carbon with a chemical shift of $\delta=68$. The relative intensity of 1.0 is equal to the combined relative intensities of a ring II carbon and a ring I $\alpha$ carbon, $\sim 0.7+\sim 0.3$.

As listed at the bottom of Table 2.3, the only published report of isomaltose ${ }^{13} \mathrm{C}$ shifts lists many overlapping resonances. Resolution is principally a function of magnetic field strength, however, and the data from which this table was prepared was published in 1973, when commercially available spectrometers operated at much lower fields. The absolute chemical shift values do not agree exactly with the ones determined here, but the listed values were corrected by Bock since he stated that the values published in the original work "are obviously too high" (Bock et al., 1984). In addition, shifts for a structure may differ by 1-2 p.p.m. depending on temperature, concentration, and reference compound used (Bock and Pederson, 1983).

\section{Distortionless Enhancement by Polarization Transfer (DEPT)}

Once the proton-decoupled carbon spectrum was acquired, DEPT ("Distortionless Enhancement by Polarization Transfer") spectra were also collected. DEPT involves transferring the magnetization of the protons to their attached carbons, resulting in an 
chemical shift: $\quad$ relative intensity (highest peak $=1$ ) assignment (ring number, carbon number)

$\begin{array}{lll}59.0 & 0.77 & \text { II6 } \\ 64.2 & 0.47 & \text { I } 6 \\ 64.3 & 0.28 & \text { I } \alpha 6 \\ 67.9 & 0.61 & \text { I } \beta 4 \\ 68.0 & 1.00 & \text { II4, I } \alpha 4 \\ 68.5 & 0.36 & \text { I } \alpha 5 \\ 69.9 & 0.49 & \text { I } 22 \\ 70.2 & 0.69 & \text { II2 } \\ 70.3 & 0.68 & \text { II5 } \\ 71.5 & 0.51 & \text { I } \alpha 3 \\ 71.6 & 0.90 & \text { II3 } \\ 72.6 & 0.59 & \text { I } \beta 2 \\ 72.8 & 0.59 & \text { I } \beta 5 \\ 74.5 & 0.60 & \text { I } 33 \\ 90.7 & 0.39 & \text { I } \alpha 1 \\ 94.6 & 0.59 & \text { I } \beta 1 \\ 96.5 & 0.68 & \text { II1 }\end{array}$

Isomaltose assignments as reported by Bock (1984)

(shift values from original reference corrected by Bock; II6 shift set to 61.6 )

61.6

66.5

70.4

72.4

72.9

74.1

75.0

76.2

92.9

96.8

98.5
II6

$\mathrm{I} \alpha 6$, I $\beta 6$

$\mathrm{II} 4, \mathrm{I} \alpha 4, \mathrm{I} \beta 4, \mathrm{I} \alpha 5$

II $2, \mathrm{I} \alpha 2$

II5

II 3, I $\alpha 3$

$\mathrm{I} \beta 2, \mathrm{I} \beta 5$

I $\beta 3$

I $\alpha 1$

I $\beta 1$

II1

Table 2.3

${ }^{13} \mathrm{C}$ chemical shifts of isomaltose

(temperature $298 \mathrm{~K}$; referenced to internal acetonitrile, $\delta=117.2$ ) 
increase in sensitivity for ${ }^{13} \mathrm{C}$ NMR. Theoretically, the sensitivity enhancement should be by a factor of ca. 6400 , because the Lamor frequency of protons is four times that of ${ }^{13} \mathrm{C}$, which means that the population difference between energy levels for protons is four times as great, the magnetic moment is four times larger, and so the signal produced by a pulse is $4 \times 4 \times 4=64$ times as great, since the signal induced in the receiver is also proportional to the precessional rate. In addition, natural abundance of ${ }^{13} \mathrm{C}$ is only $1 \%$ of total carbon. Of use in the case of isomaltose (since neither sample quantity nor solubility are a problem) is the fact that DEPT can show the number of protons attached to each carbon, so \#6 carbons in this case can be distinguished unambiguously from the other carbons. Three DEPT spectra are shown in Fig. 2.11. The bottom trace, DEPT 45, shows all protonated carbons, which in the case of isomaltose is all of the carbons (compare to Fig. 2.10). Through multiplequantum filtration (see references at the beginning of the chapter for details), the DEPT 90 and DEPT 135 'sort' the carbons according to the numbers of attached protons. The DEPT 90 shows only carbons with one proton attached, while the DEPT 135 shows - $\mathrm{CH}$ and $\mathrm{CH}_{3}$ carbons pointing up, and inverts $-\mathrm{CH}_{2}$ carbons. By comparing the DEPT 45, 90, and 135 , the identity of the \#6 carbons is readily confirmed: as expected, they are the three most upfield carbons.

\section{Heteronuclear Correlation Spectroscopy (HETCOR)}

The next step was acquisition of a HETCOR, heteronuclear correlated experiment (Fig. 2.12) in which the connectivities of the protons and carbons in a structure can be established. In this experiment, magnetization is transferred between carbons and their attached protons. As the two axes (F2, F1) of the matrix represent carbon and proton frequencies, respectively, the matrix is not symmetrical, and none of the crosspeaks is redundant; each represents a direct connection between a carbon and its attached proton(s). Since the position of the anomeric protons, as well as the \#2, \#3, and some of the \#4 protons were established in the COSY, these peaks could be used to confirm the position of the corresponding carbons. The position of the \#6 carbons, on the other hand, was known from the DEPT spectra, and through the HETCOR the corresponding protons, and therefore also the crosspeaks to the \#5 protons, could be established. By working with the spectra (and using enlargements and cross-sections), the isomaltose structure was assigned as shown (Fig. 2.12, Table 2.4). 
(a)

(b)

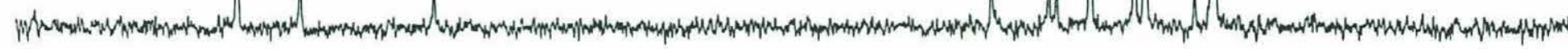

(c)

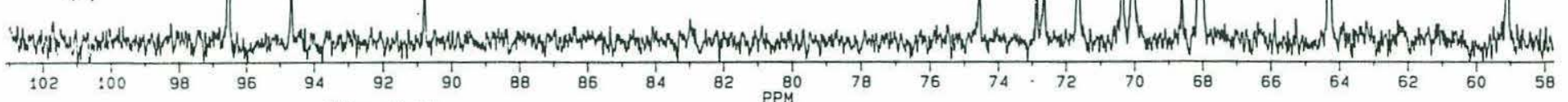

Fig. 2.11

DEPT experiments for isomaltose: (a) DEPT $135^{\circ}$, (b) DEPT $90^{\circ}$, (c) DEPT $45^{\circ}$.

Temperature $=298 \mathrm{~K}$, internal standard: acetonitrile $(\delta=117.2)$. 


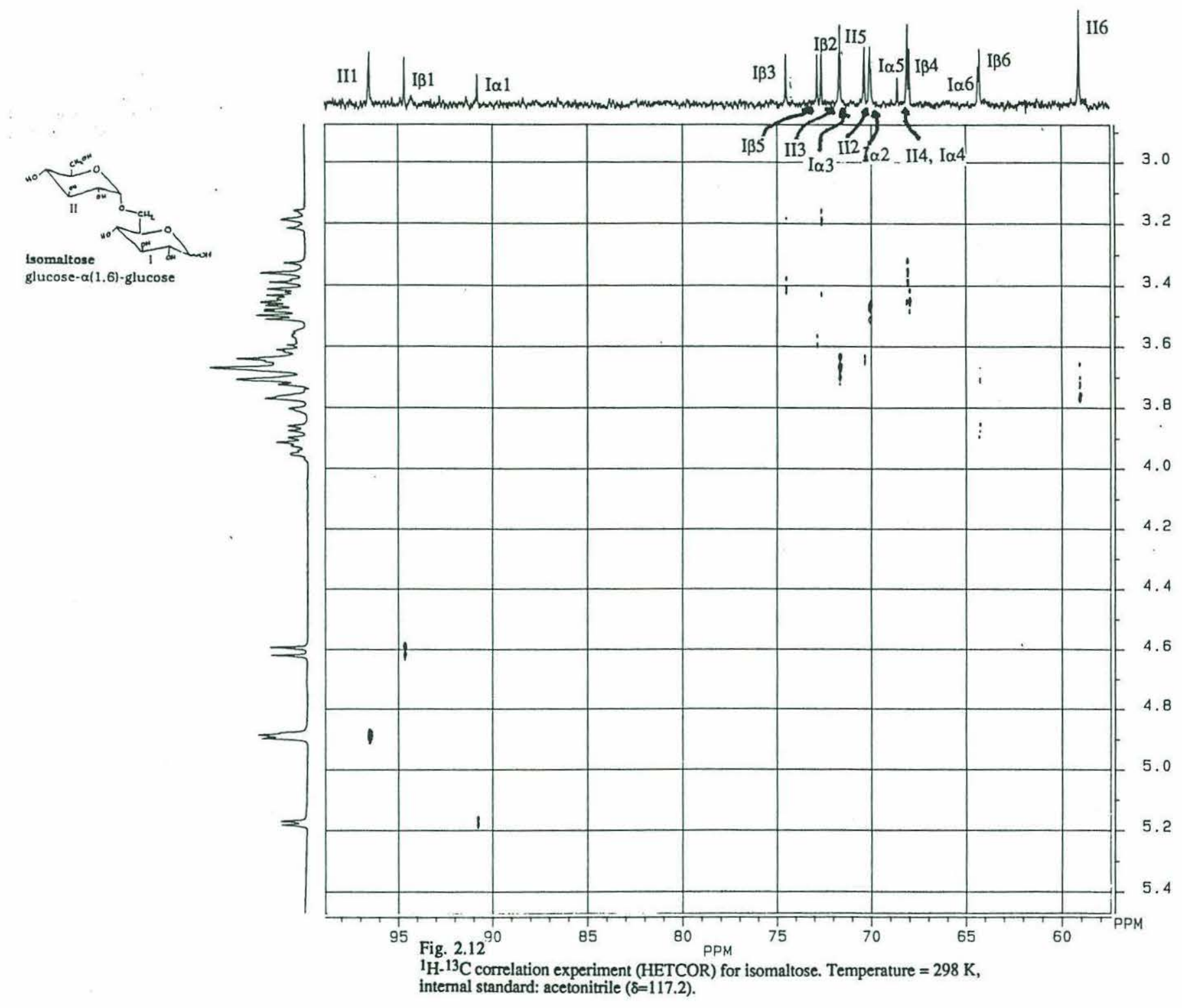


chemical shift:

\section{assignment (ring number,} proton number)

3.21
3.35

3.41

3.45

3.48

3.52

3.57

3.64

$3.66 * *$

$3.66 * *$

$3.68 * *$

3.70

$3.71 *$

3.97

4.65

4.90

5.20

I 32

II4

I 34

I 33

$\mathrm{I} \alpha 2$

II2

I 35

II5

I $\beta 6$

$\mathrm{I} \alpha 4$

I $\beta 6^{\prime}$

II3

II6, 6'

I $\alpha 3$

I 31

II1

$\mathrm{I} \alpha 1$

not determined: $\quad \mathrm{I} \alpha 5, \mathrm{I} \alpha 6$

* midpoint of HETCOR crosspeak; 6 and 6' protons not individually resolved

** approximate chemical shift (low resolution crosspeak)

Table 2.4

${ }^{1} \mathrm{H}$ chemical shifts of isomaltose

(temperature $298 \mathrm{~K}$; referenced to HOD, $8=4.8$ ) 


\section{Maltose and Maltotriose}

A comparision of the COSY of maltose (glucose- $\alpha(1,4)$-glucose; Fig. 2.13 ) and isomaltose (glucose- $\alpha(1,6)$-glucose; Fig. 2.8 ) demonstrates that linkage position, as well as anomeric linkage, significantly affects solution conformation and therefore the appearance of the spectrum. The 1-D trace above the 2-D COSY of maltotriose (Fig. 2.14) illustrates the extent to which individual proton resonances coalesce in the region at $\delta=3.5-4.0$ with the addition of just one monosaccharide. Using a variety of one- and two-dimensional experiments, Morris and Hall (Morris and Hall, 1982) completely assigned both the carbon and the proton spectra of these structures.

\section{Polysaccharides}

As illustrated above, increasing the size and complexity of a carbohydrate decreases the resolution of its NMR spectrum. Studies of high-molecular weight polysaccharides are also complicated by the low solubility of many polymers. The types of experiments which can be run, and the information which can be gleaned from these experiments, is therefore often limited by the solubility of the material. Spectra of three linear glucose polymers were collected in order to characterize them for use in carbohydrate degradation experiments (Chapter 5), and to compare them to the spectra of structurally related di- and oligosaccharides. In addition, running NMR experiments and optimizing various experimental parameters on these known polysaccharides was an important step in characterizing natural polysaccharides (Chapter 3).

Three linear glucose polysaccharides were obtained from U.S. Biochemicals: amylose, an $\alpha(1,4)$-linked glucose polysaccharide with a molecular weight of $10-50 \mathrm{kD}$, laminarin, a $\beta(1,3)$-linked glucose polysaccharide of 5-6 kD, and pullulan, a polymer of $200 \mathrm{kD}$ which is made up of $\alpha(1,6)$-linked maltotriose $(\alpha(1,4))$ units. These three polymers graphically illustrate the extent to which solubility is dependent not only on molecular size, but also upon linkage type and position, and, by extension, upon solution conformation. All three polymers are linear, all are composed entirely of glucose, and pullulan and amylose differed only in that pullulan is at least four times larger, and every fourth linkage in pullulan is $(1,6)$ instead of $(1,4)$. Pullulan, the largest of the polymers, was far more soluble than amylose: more than $50 \mathrm{mg}$ of pullulan, and less than $1 \mathrm{mg}$ of amylose, for example, could be dissolved in $0.5 \mathrm{~mL} \mathrm{D}_{2} \mathrm{O}$. Laminarin, the smallest of the polymers, was more soluble than amylose, but far less soluble than pullulan. 


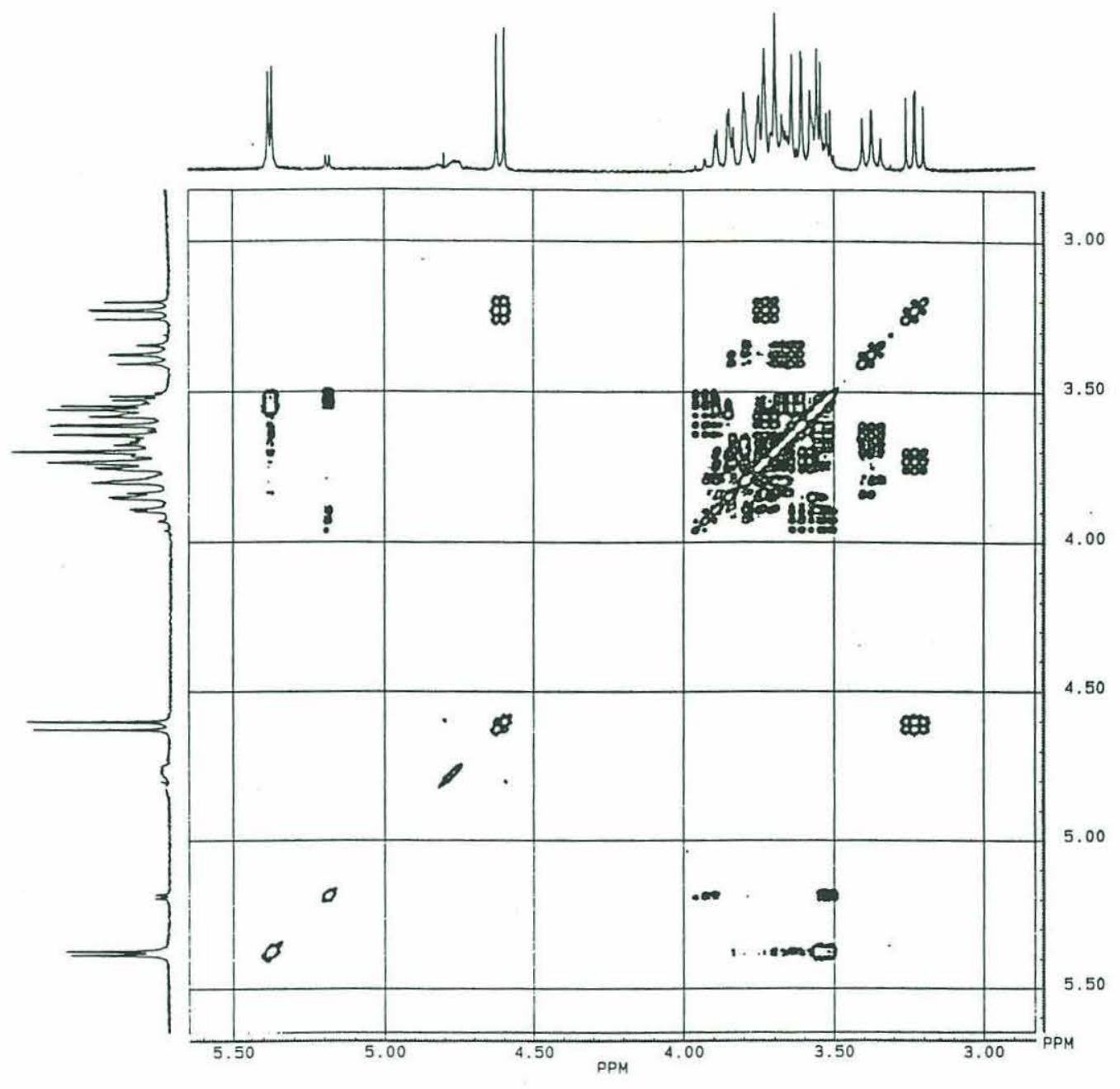

Fig. 2.13

${ }^{1} \mathrm{H}$ COSY $45^{\circ}$ of maltose (HOD resonance set to $\delta=4.8$ ). 


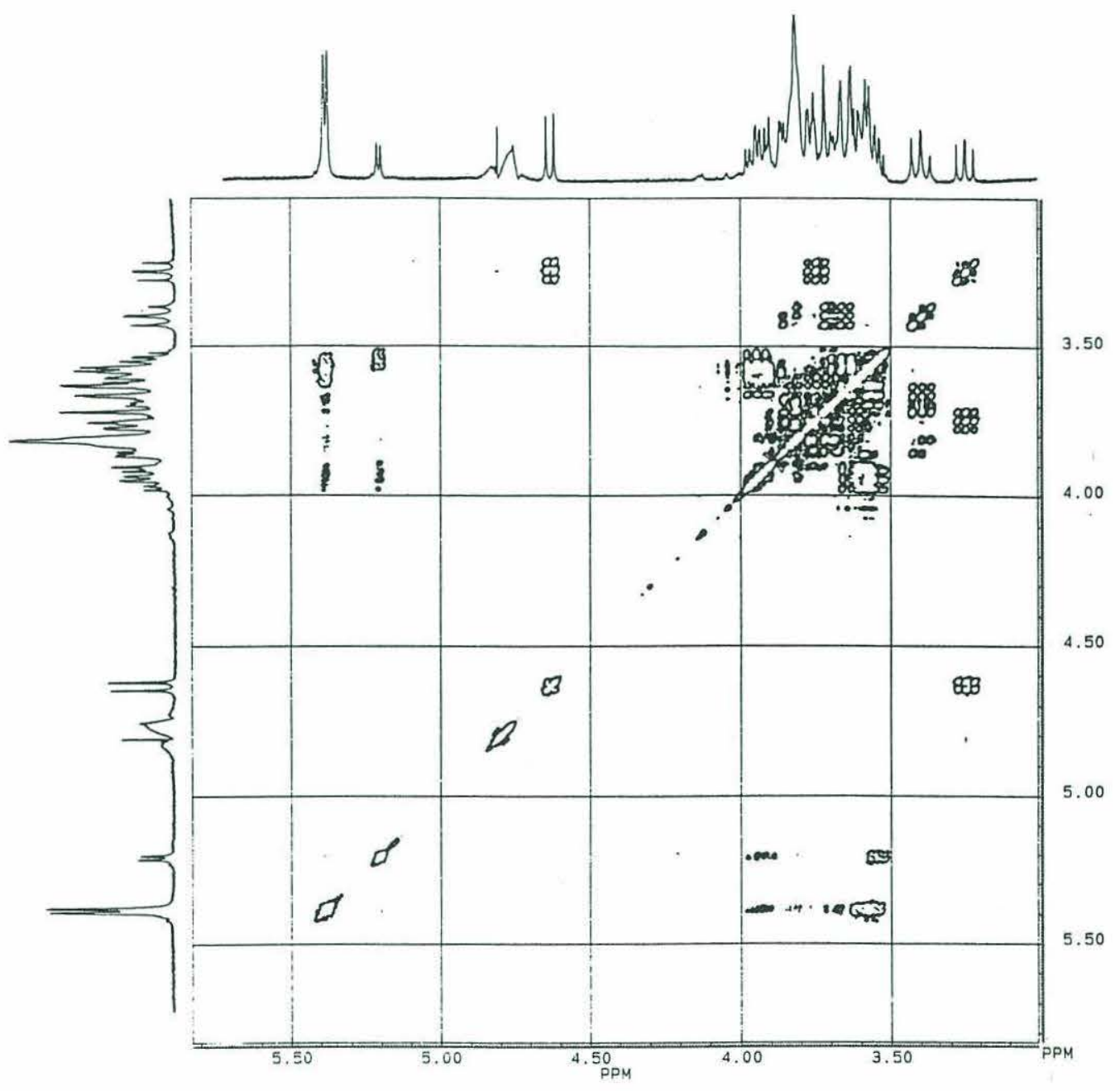

Fig. 2.14

${ }^{1} \mathrm{H} \operatorname{COSY} 45^{\circ}$ of maltotriose (HOD resonance set to $\delta=4.8$ ). 


\section{Amylose}

A comparison of the 1-D proton spectrum of amylose (Fig. 2.15) with the spectra of glucose, maltose, and maltotriose (Figs. 2.5, 2.13, and 2.14) shows the extent to which the resolution in a polysaccharide decreases with molecular weight. Only the large anomeric peak and the triplet at $\delta=3.4$ (due to the \#4 protons) are well-resolved. ${ }^{13} \mathrm{C}$ spectrum (Fig. 2.16), showing six resonances, was acquired in approximately 24 hours of run-time with ca. $5 \mathrm{mg}$ of material at a temperature of $60^{\circ} \mathrm{C}$. This probably represents the practical minimum in terms of sample quantity for a ${ }^{13} \mathrm{C}$ spectrum of a polysaccharide; for branched or heteropolysaccharides, more material would be required, because there would be a greater variety of carbon types present and effective concentrations of each carbon type would be accordingly lower.

\section{Laminarin}

The resolution of individual protons in a polysaccharide is not nearly as high as for mono- and oligosaccharides, but 1D proton spectra still provide characteristic patterns for a polymer. The proton spectrum of laminarin (Fig. 2.17) distinctly differs from that of amylose. In particular, the region between $\delta=3.4-4.0$ shows several distinctive groupings. In addition, the anomeric resonance at $\delta=4.85$ is one of the few examples of a $\beta$-anomer which resonates downfield of the HOD peak (here set to $\delta=4.8$ ).

\section{Pullulan}

The COSY spectrum of pullulan (Fig. 2.18) clearly differs from the other glucose polymers. The two anomeric peaks at $\delta=5.33$ and 5.38 are the two $\alpha(1,4)$ linkages within the maltotriose units, while the $\alpha(1,6)$ anomer is at $\delta=4.93$. The crosspeaks within the COSY matrix provide clear connections to the $\# 2$ protons, but tracing the proton network through the COSY would be difficult. Because of pullulan's high solubility, however, additional experiments are possible. Fig. 2.19 is the ${ }^{13} \mathrm{C}$ spectrum of $50 \mathrm{mg}$ of pullulan. Eighteen resonances, representing all of the carbon types in the sample, are clearly distinguishable.

No attempt was made to completely assign the proton and carbon spectra, since proton and carbon shifts have been reported for pullulan (McIntyre et al., 1990). 


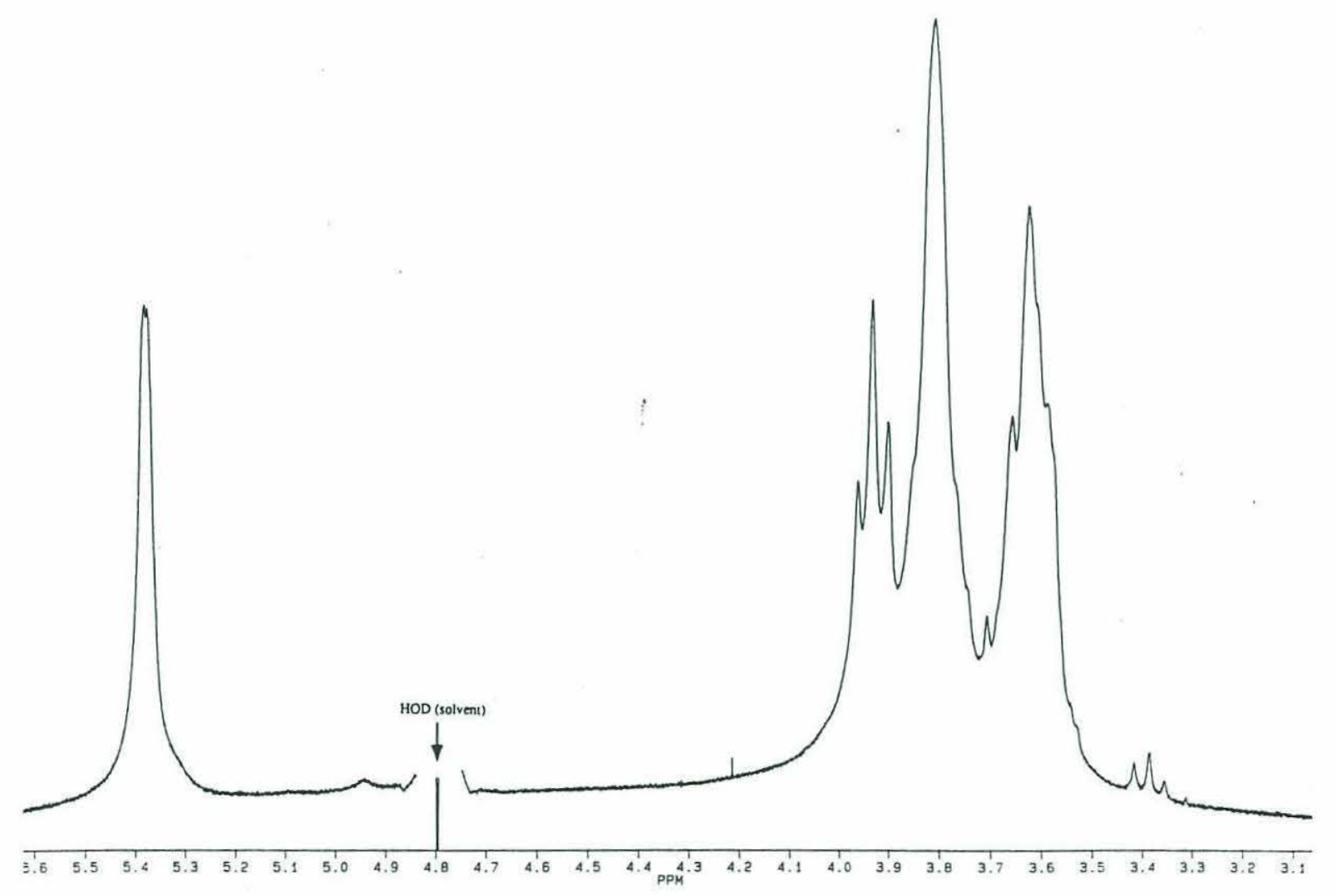

Fig. 2.15

${ }^{1} \mathrm{H}$ spectrum of amylose (HOD resonance set to $\delta=4.8$ ). 

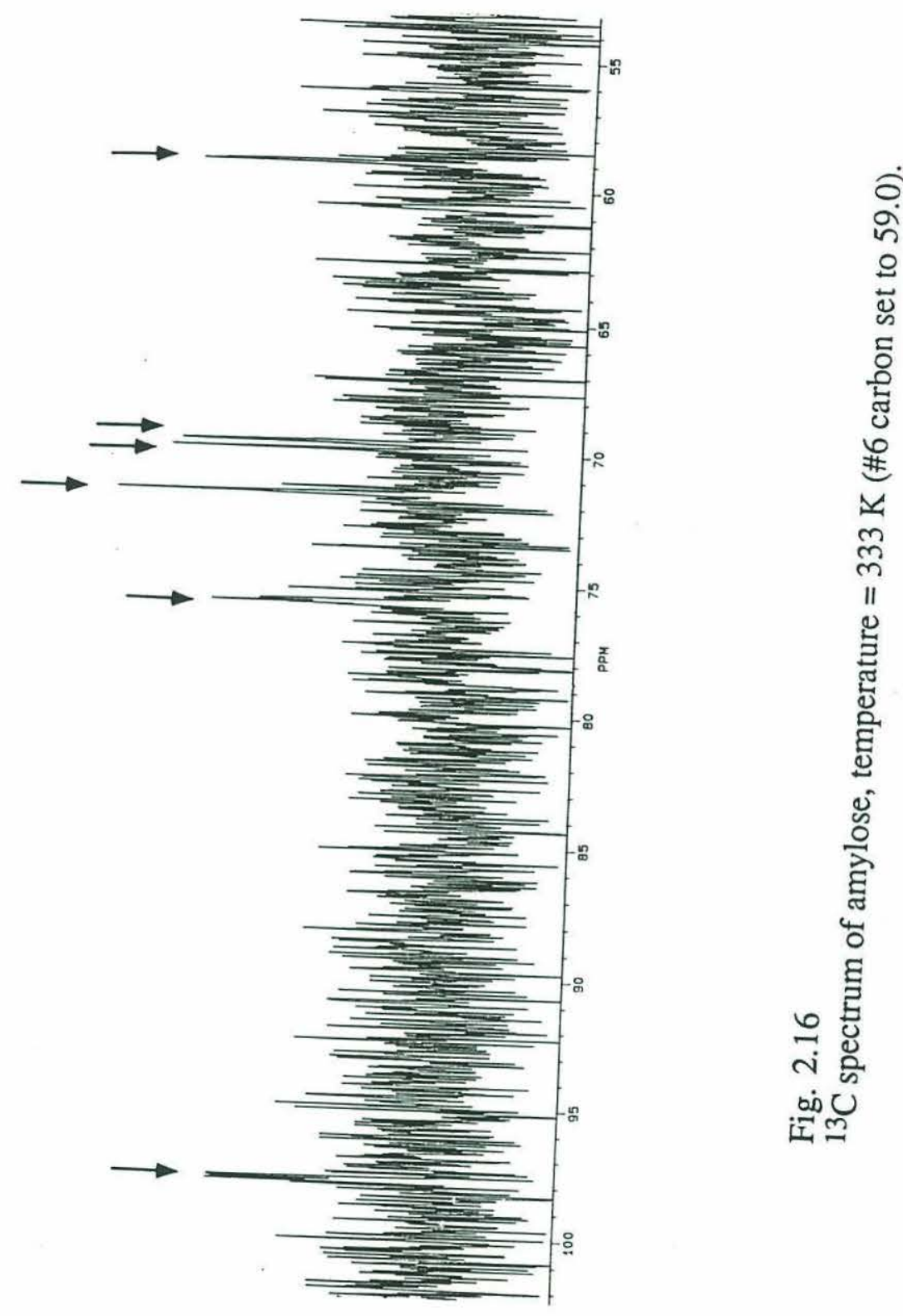


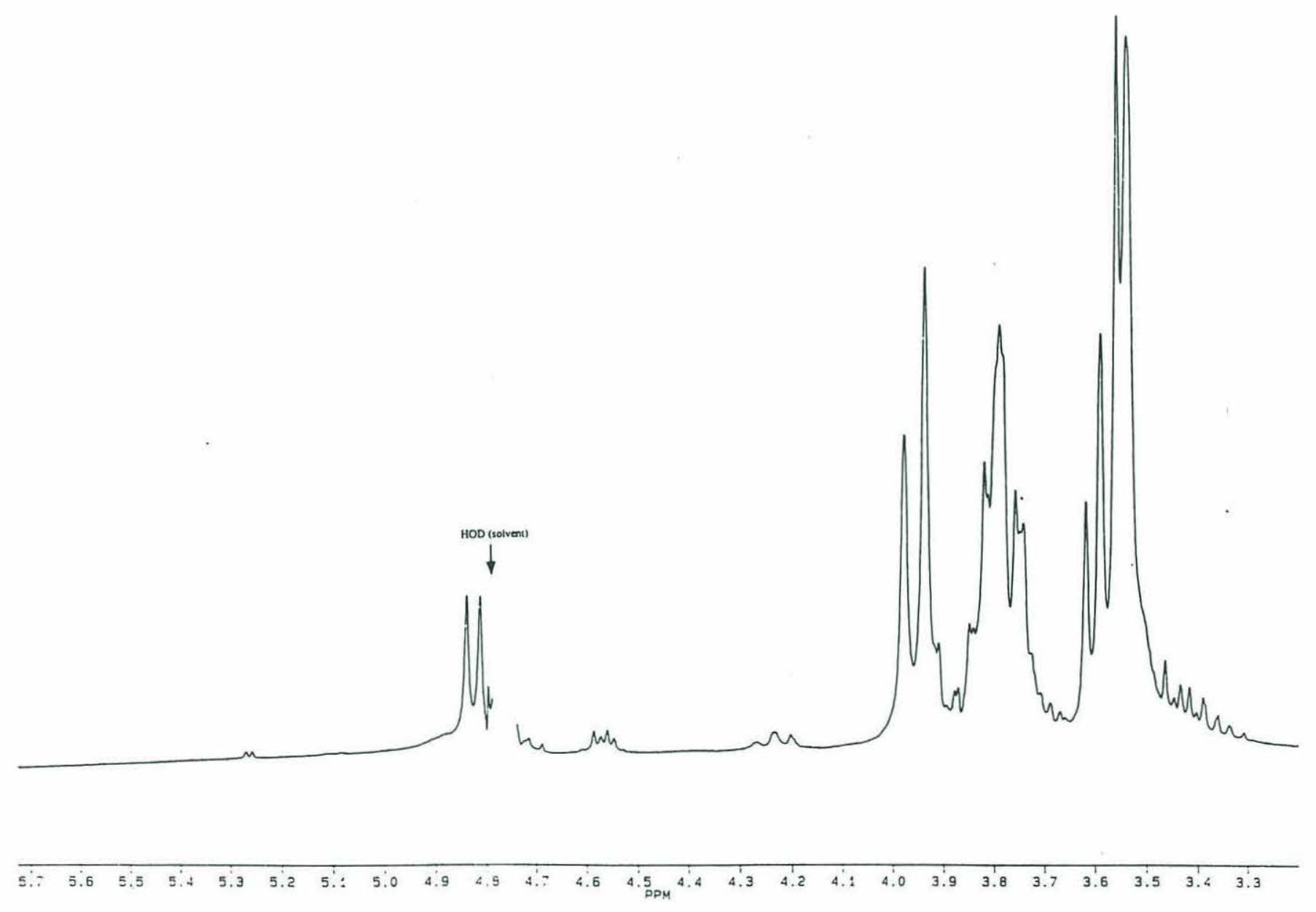

Fig. 2.17

${ }^{1} \mathrm{H}$ spectrum of laminarin (HOD resonance set to $\delta=4.8$ ). 


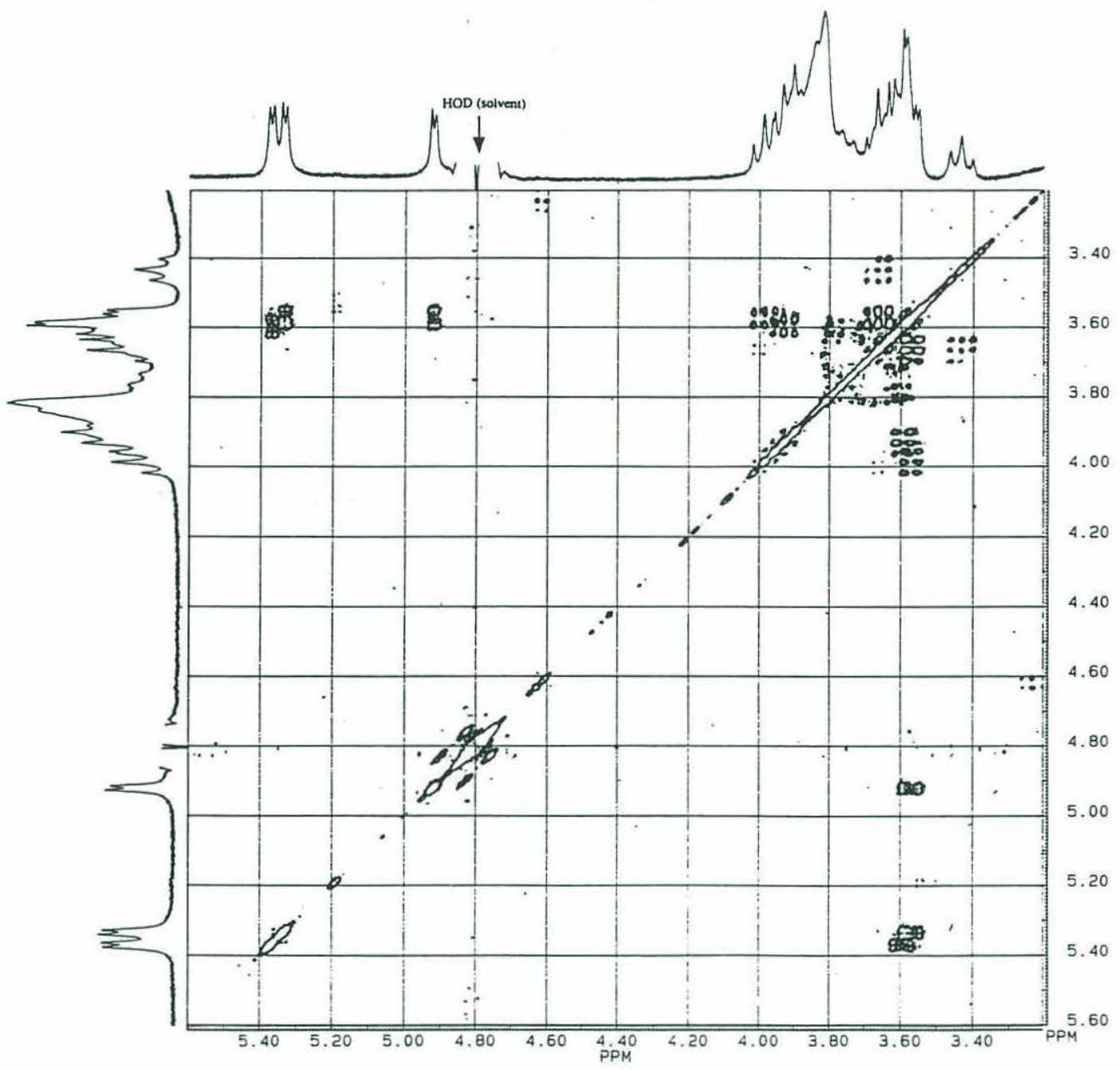

Fig. 2.18

${ }^{1} \mathrm{H}$ COSY of pullulan (HOD resonance set to $\delta=4.8$ ). 


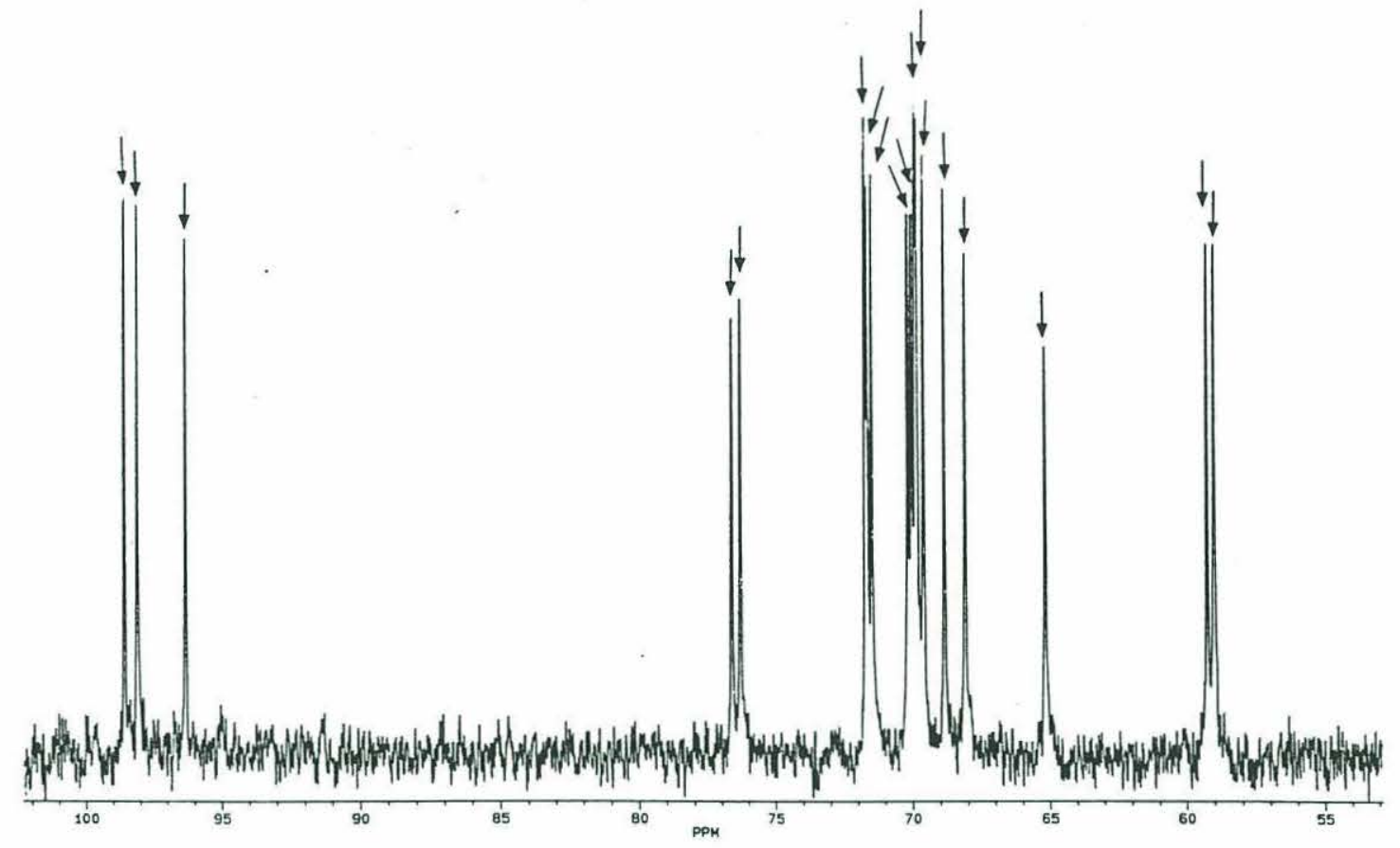

Fig. 2.19

${ }^{13} \mathrm{C}$ spectrum of pullulan (ca. $50 \mathrm{mg}$ in $0.5 \mathrm{~mL}$ ); temperature $=298 \mathrm{~K}$, pulse width $60^{\circ}$, internal standard: acetonitrile $(\delta=117.2)$. 
One intriguing aspect was pursued, however, which illustrates the fact that spectrometer and sample conditions can affect sample resolution. The published ${ }^{13} \mathrm{C}$ shifts did not include eighteen distinct carbon resonances; three resonances were assigned a shift value of $\delta=72.1$, and for one $\# 2$ carbon, shift was not determined. The published data were obtained with a $400 \mathrm{MHz}$ NMR (100 MHz for carbon), which should normally provide better resolution than the $300 \mathrm{MHz}$ ( $75 \mathrm{MHz}$ for carbon) spectrometer used to obtain the spectrum in Fig. 2.19. Better resolution with the lower-field instrument could be due to lower sample concentration, since a viscous solution results in line broadening and overlap. In order to assign the newly-resolved resonances, a HETCOR experiment was run. Carbon-proton crosspeaks were obtained for all but the weakest \#6 resonance at $\delta=67.7$ (Fig. 2.20). The assignment of the carbons between $\delta=68.5$ and 70.5 was the focus: even though absolute chemical shifts change with sample temperature and concentration, relative shifts should remain the same, so the order of shifts should be the same as reported in (McIntyre et al., 1990). The reported proton shifts showed that all three \#2 protons were found in the region from $\delta=3.6-3.65$, while all three \#5 protons had shifts of $\delta=3.9-3.92$ (at a temperature of $60^{\circ} \mathrm{C}$; absolute shifts at $25^{\circ} \mathrm{C}$ are different). Inspection of Fig. 2.21 shows that crosspeaks for the carbon resonances clustered between $\delta=68.5$ and 70.5 fell neatly into two groups. The crosspeak at $\delta=68.83$ was known to be a $\# 5$ carbon, with a corresponding proton crosspeak between $\delta=3.79-3.89$. Two crosspeaks with the same proton shift corresponded to the carbons at $\delta=69.83$ and 69.89 , which therefore must be the other two \#5 carbons. Similarly, the carbons at $\delta=69.58$ and 70.02 show proton crosspeaks in the same region as the carbon at $\delta=70.15$, which is known to be a $\# 2$ carbon. The "missing" \#2 carbon (the \#2 carbon in the first ring of the maltotriose group) therefore has a shift of $\delta=70.02$ in this spectrum.

The pullulan spectra probably represent a 'best case', in terms of resolution and solubility for a high molecular-weight polysaccharide, while amylose represents a minimum among soluble polysaccharides. The experiments used here are only a small number of the many possibilities currently available with most modern NMR spectrometers; specific questions of linkage position and solution conformation can be further investigated with a variety of nOe (nuclear Overhauser effect) and relay-COSY experiments, for example. In any case, pictorial representations of carbohydrates clearly do not fully reveal the real differences between closely related structures in terms of their threedimensional conformations in solution. These differences manifest themselves in terms of physical properties such as solubility and hydrodynamic volume (Kennedy et al., 1988), and may play a significant role in determining the accessibility of a polysaccharide to 
bacterial exoenzymes; such factors may help determine the relative reactivities of natural polysaccharides in marine environments. 


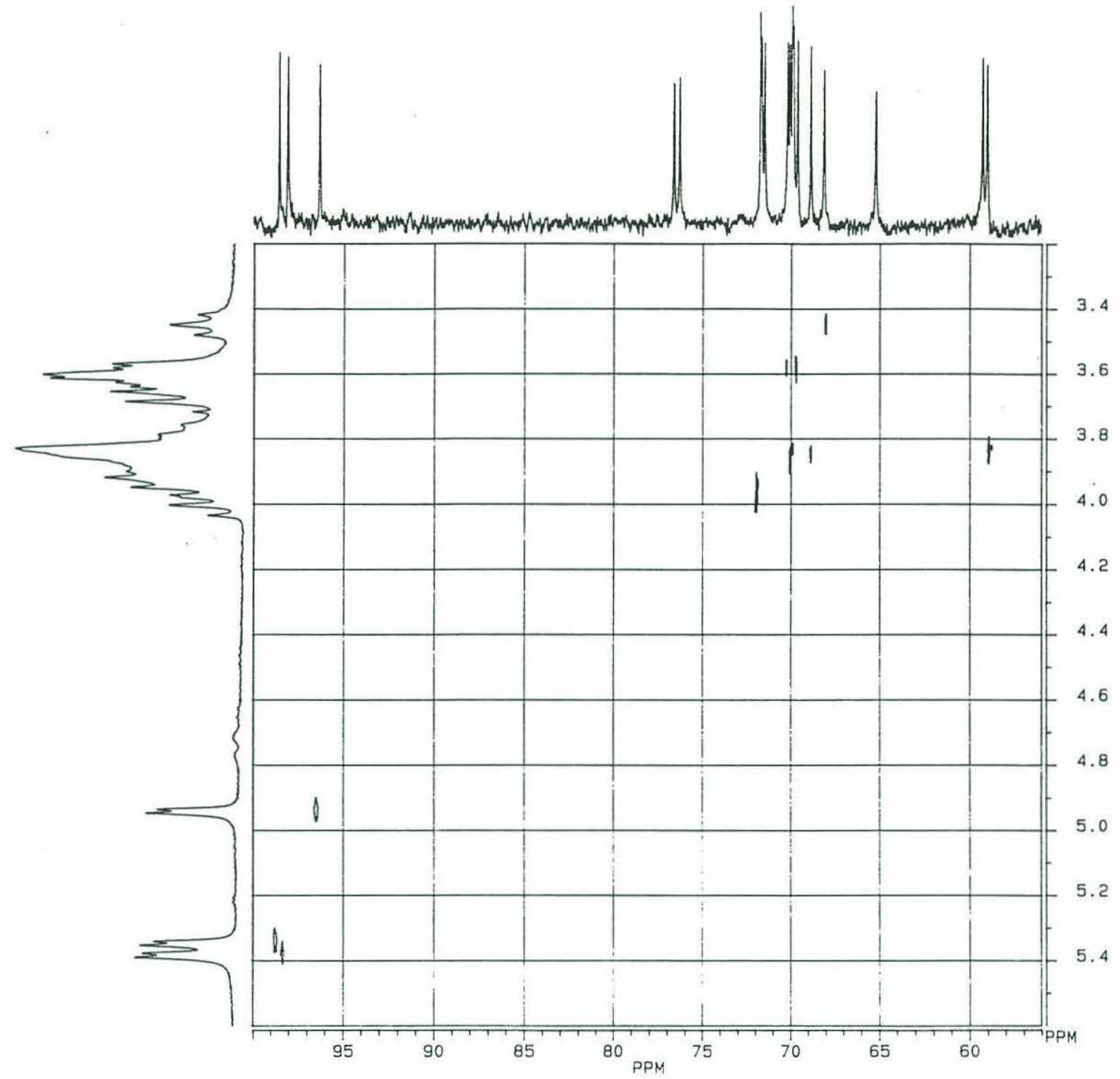

Fig. 2.20

${ }^{1} \mathrm{H}-{ }^{13} \mathrm{C}$ correlation experiment $(\mathrm{HETCOR})$ of pullulan; temperature $=298 \mathrm{~K}$, internal standard: acetonitrile $(\delta=117.2)$. 


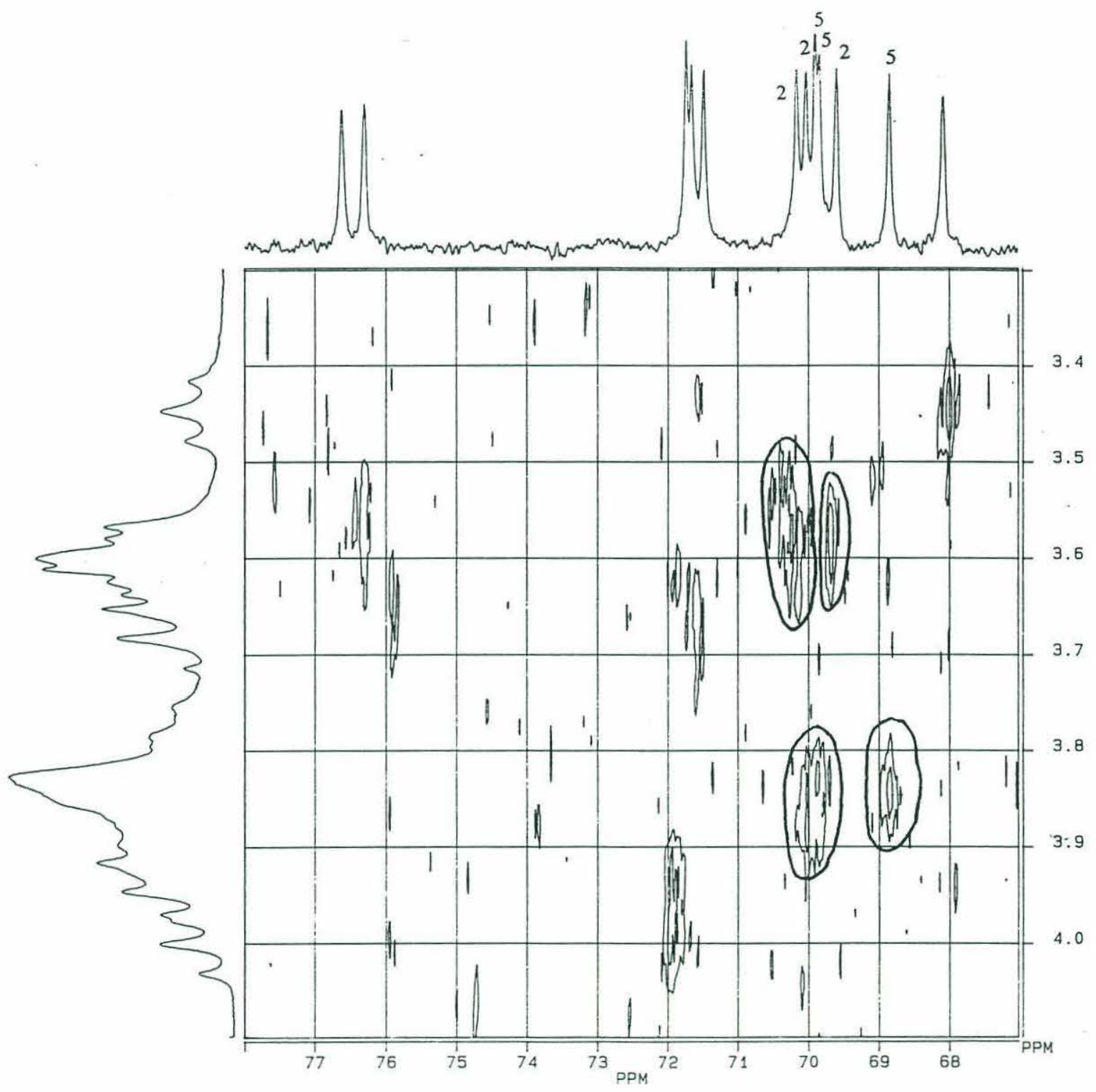

Fig. 2.21

Expansion of pullulan HETCOR. 


\section{References}

Backman, I., B. Erbing, P.-E. Jansson and L. Kenne (1988). N.m.r. and conformational studies of some 1,4-linked disaccharides. J. Chem. Soc. Perkin Trans. I : 889-898.

Backman, I., P.-E. Jansson and L. Kenne (1990). Synthesis, NMR and conformational studies of some 1,4-linked disaccharides. J. Chem. Soc. Perkin Trans. I : 1383-1388.

Backman-Marklund, I., P.-E. Jansson, B. Lindberg and J. Henrichsen (1990). Structural studies of the capsular polysaccharide from Streptococcus pneumoniae type 7A. Carbohydr. Res. 198: 67-77.

Baumann, h., B. Erbing, P.-E. Jansson and L. Kenne (1989). N.m.r. and conformational studies of some 3-O-, 4-O-, and 3,4-Di-O-glycopyranosyl-substituted methyl $\alpha$-Dgalactopyranosides. J. Chem. Soc. Perkin Trans. I : 2153-2165.

Baumann, H., P.-E. Jansson and L. Kenne (1988). N.m.r. and conformational studies on some 1,3-linked disaccharides. J. Chem. Soc. Perkins Trans. I : 209-217.

Benner, R., P. G. Hatcher and J. I. Hedges (1990). Early diagenesis of mangrove leaves in a tropical estuary: bulk chemical characterization using solid-state 13C NMR and elemental analyses. Geochim. Cosmochim. Acta 54: 2003-2013.

Benner, R., J. D. Pakulski, M. McCarthy, J. I. Hedges and P. G. Hatcher (1992). Bulk chemical characteristics of dissolved organic matter in the ocean. Science 255: 1561-1564.

Berkaloff, C., E. Casadevall, C. Largeau, P. Metzger, S. Peracca and J. Virlet (1983). The resistant polymer of the walls of the hydrocarbon-rich alga Botryococcus braunii. Phytochem. 22: 389-397.

Bock, K. and C. Pederson (1983). Carbon-13 nuclear magnetic resonance spectroscopy of monosaccharides. Adv. Carbo. Chem. Biochem. 41: 27-66.

Bock, K., C. Pederson and H. Pederson (1984). Carbon-13 nuclear magnetic resonance data for oligosaccharides. Adv. Carbo. Chem. Biochem. 42: 193-225.

Bock, $\mathrm{K}$. and $\mathrm{H}$. Thogersen Nuclear magnetic resonance spectroscopy in the study of mono- and oligosaccharides. : 1-57.

Buddrus, J., P. Burba, H. Herzog and J. Lambert (1989). Quantitation of partial structures of aquatic humic substances by one- and two-dimensional solution ${ }^{13} \mathrm{C}$ nuclear magnetic resonance spectroscopy. Anal. Chem. 61: 628-631.

Cassels, F., H. M. Fales, J. London, R. W. Carlson and H. van Halbeek (1990). Structure of a streptococcal adhesin carbohydrate receptor. J. Biol. Chem. 265: 1412714135 .

Clore, G. M. and A. M. Gronenborn (1991). Structures of larger proteins in solution: three- and four-dimensional heteronuclear NMR spectroscopy. Science 252: 1390-1399.

DeBruyn, A. (1991). 1H NMR chemical shift information on the conformation of the glycosidic bond in disaccharides. J. Carbo. Chem. 10(2): 159-180. 
Derome, A. E. (1987). Modern NMR Techniques for Chemistry Research. Oxford, Pergamon Press.

Doco, T., J.-M. Wieruszeski, B. Fournet, D. Carcano, P. Ramos and A. Loones (1990). Structure of an exocellular polysaccharide produced by Streptococcus thermophilus. Carbohydr. Res. 198: 313-321.

Fontaine, T., J.-M. Wieruszeski, F. Talmont, M.-H. Saniez, P. Duflot, J.-B. Leleu and B. Fournet (1991). Exopolysaccharide structure from Bacillus circulans. Eur. J. Biochem. 196: $107-113$.

Friebolin, H. (1991). Basic One- and Two-Dimensional NMR Spectroscopy. Weinham, $\mathrm{VCH}$.

Geresh, S., O. Dubinsky, S. Arad, D. Christiaen and R. Glaser (1990). Structure of the 30 -( $\alpha$-D-glucopyranosyluronic acid)-L-galactopyranose, an aldobiouronic acid isolated from the polysaccharides of various unicellular red algae. Carbohydr. Res. 208: 301-305.

Gorin, P. A. J. (1981). Carbon-13 nuclear magnetic resonance spectroscopy of polysaccharides. Adv. Carbo. Chem. Biochem. 38: 13-97.

Ikura, M. and K. Hikichi (1987). Two-dimensional 1H-N.M.R. studies of cellooligosaccharides: the utility of multiple-relay chemical-shift-correlated spectroscopy. Carbo. Res. 163: 1-8.

Jansson, P. E., L. Kenne and E. Schweda (1988). N.M.R. and conformational studies of the methyl glycosides of some 1,2- and 1,3-linked disaccharides. J. Chem. Soc. Perkins Trans. I : 2729-2736.

Jaseja, M., A. S. Perlin, O. Dubinsky, D. Christiaen, S. Arad and R. Glaser (1989). N.m.r. structure determination of 3-0-( $\alpha$-D-glucopyranosyluronic acid)-Lgalactopyranose, an aldobiuronic acid isolated from the unicellular red alga Rhodella reticulata. Carbohydr. Res. 186: 313-319.

Kennedy, J. F., D. L. Stevenson and C. A. White (1988). The behavior and identification of a range of starch and starch-related oligosaccharides series on gel permeation chromatography as a function of their molecular shapes. Starch/Starke 40: 396-404.

Knutsen, S. H., D. E. Myslabodski and H. Grasdalen (1990). Characterization of carrageenan fractions from Norwegian Furcellaria lumbricalis (Huds.) Lamour. by $1 \mathrm{H}-$ NMR spectroscopy. Carbohydr. Res. 206: 367-372.

Lambert, J., P. Burba and J. Buddrus (1992). Quantification of partial structures in aquatic humic substances by volume integration of two-dimensional $13 \mathrm{C}$ nuclear magnetic resonance spectra. Comparision of one- and two-dimensional techniques. Mag. Res. Chem. 30(3): 221-227.

Martin, G. E. and A. S. Zektzer (1988). Two-Dimensional NMR Methods for Establishing Molecular Connectivity. Weinham, VCH.

McIntyre, D. D., C. Ho and H. J. Vogel (1990). One-dimensional nuclear magnetic resonance studies of starch and starch products. Starch/Staerke 7: 260-267. 
McIntyre, D. D. and H. J. Vogel (1990). Two-dimensional nuclear magnetic resonance studies of starch and starch products. Starch/Staerke 42(8): 287-293.

McIntyre, D. D. and H. J. Vogel (1991). Nuclear magnetic resonance studies of homopolysaccharides related to starch. Starch 43(2): 69-76.

Morris, G. A. and L. D. Hall (1982). Experimental chemical shift correlation maps from heteronuclear two-dimensional nuclear magnetic resonance spectroscopy. II: Carbon-13 and proton chemical shifts of $\alpha$-D-glucopyranose oligomers. Can. J. Chem. 60: 24312441.

Saito, H., J. Yamada, Y. Yoshioka, Y. Shibata and T. Erata (1991). Evidence of three distinct conformations-single chain, single helix, and triple helix-of $(1,3)-\beta$-D-xylan in the solid and intact frond of green algae as studied by ${ }^{13} \mathrm{C}-\mathrm{NMR}$ spectroscopy. Biopolymers 31: $933-940$.

Varum, K. M., J. K. Bjarne and S. Myklestad (1986). Structure of a food-reserve $\beta$-Dglucan produced by the haptophyte alga Emiliania huxleyi (Lohmann) Hay and Mohler. Carbo. Res. 152: 243-248.

Weis, W. I., K. Drickamer and W. A. Hendrickson (1992). Structure of a C-type mannose-binding protein complexed with an oligosaccharide. Nature 360: 127-134.

Wershaw, R. L., D. J. Pickney, E. C. Llaguno and V. Vincente-Beckett (1990). NMR characterization of humic acid fractions from different Philippine soils and sediments. Anal. Chim. Acta 232: 31-42.

Zelibor, J. L., jr., L. Romankew, P. G. Hatcher and R. R. Colwell (1988). Comparative analysis of the chemical composition of mixed and pure cultures of green algae and their decomposed residues by $13 \mathrm{C}$ nuclear magnetic resonance spectroscopy. Appl. Environ. Microb. 54(4): 1051-1060. 


\section{Chapter Three: Plankton Carbohydrates}

\section{Introduction}

Surprisingly little is known about the macromolecular structure of the material produced through photosynthesis. The proportion of photosynthetically-fixed carbon which is incorporated into phytoplankton lipids, proteins, and carbohydrates under a range of growth conditions has been studied using ${ }^{14} \mathrm{C}$ incorporation as a tracer (Morris et al., 1974; Hitchcock, 1978; Morris, 1981; Hitchcock et al., 1986; Smith et al., 1987). In these studies, the relative quantities of cellular components in each ${ }^{14} \mathrm{C}$ - labeled pool (low molecular weight metabolites, proteins, lipids, and polysaccharides) were determined using a sequential extraction method. The method provides an estimate of the distribution of cellular carbon among major classes of biomolecules, but it does not provide any structural information about the material in each fraction. In addition, the classification of cellular components using operationally-defined extraction techniques is problematic and may inaccurately reflect actual cellular composition of phytoplankton. Smith et al. (1987), for example, found that laminarin-like polysaccharides were extracted in the 'low molecular weight metabolite' fraction, and Smucker and Dawson (1986) demonstrated that chitin (a polysaccharide of $\mathrm{N}$-acetyl glucosamine) is found in the 'protein' fraction of cells.

Studies using carbohydrate-specific detection methods have demonstrated that much of the carbon fixed by phytoplankton during photosynthesis is converted to carbohydrates (Parsons et al., 1961; Handa, 1969; Haug et al., 1973; Mykelstad, 1977; Hama, 1988), which function as energy reserves, membrane components (Lee, 1980), and as receptors in molecular recognition and communication processes (Bishop and Jennings, 1982). Some phytoplankton also excrete carbohydrates; these exudates may be an important energy source for heterotrophs (Fogg, 1977; Cole et al., 1982; Iturriaga and Zsolnay, 1983; Sundh, 1989). A comprehensive compilation of literature on phytoplankton carbohydrates, including species studied, carbohydrate characterization methods, and abbreviated results is included as an appendix to the thesis. This chapter begins with a short summary of previous work on phytoplankton cellular and excreted carbohydrates. The results of a survey of the carbohydrate composition of four species of phytoplankton are then briefly presented. The remainder of the chapter is devoted to the detailed characterization of carbohydrates of the marine cyanobacterium Synechococcus WH7335; these carbohydrates served as substrates for bacteria in degradation experiments discussed in Chapter 5. 


\section{Phytoplankton carbohydrates}

Characterization of phytoplankton carbohydrates has generally been limited to determinations of monosaccharide concentration and composition. Even these measurements are imprecise, since conditions sufficient to liberate some fractions of carbohydrates result in the destruction of more labile carbohydrates, while mild extraction techniques can result in incomplete extraction of hydrolysis-resistant carbohydrates such as uronic acids (Henrichs, 1991). For a few types of phytoplankton carbohydrates, additional structural information is available. Polysaccharides which function as energy reserves are the most completely characterized phytoplankton carbohydrates. In a number of studies, linkage position and orientation, and estimates of molecular weights of storage polysaccharides have been made. Linkage position, and in some cases linkage orientation, has also been determined for cell wall components and excreted polysaccharides of a few species of phytoplankton. In general, however, little is known about the gross structure, molecular weight, linkage position, or anomeric linkage of the carbohydrates produced by marine phytoplankton.

Studies of monosaccharide composition and concentration have demonstrated that the monosaccharide components of phytoplankton energy storage products, membranes, and excreted polysaccharides differ significantly. Energy reserves, for example, are typically glucose-rich homopolysaccharides which can be readily converted through the glycolytic pathway to energy and to carbon building blocks for other cellular components. A number of different glucose polymers have been identified in marine phytoplankton. Laminarin, a linear glucose polysaccharide with $\beta(1,3)$ linkages (Handa, 1969; Haug and Myklestad, 1976), $\beta(1,6)$ glucose polymers with $\beta(1,3)$ branches (Varum et al., 1986), and branched $\beta(1,3)$ glucans (Paulsen and Myklestad, 1978) have been identified in diatoms and prymnesiophytes. Starch, a mixture of a linear $\alpha(1,4)$ polysaccharide (amylose) and an $\alpha(1,4)$ polysaccharide with $\alpha(1,6)$ branches (amylopectin), is an energy storage polymer which has been found in dinoflagellates and green algae (Lee, 1980).

Carbohydrates in cell walls form fibrous networks which provide structural support. These networks may be glucose-rich if they are made of cellulose $(a)(1,4)$ glucose polymer), but mannan (a polymer of mannose) and xylan (xylose polymer) fibers are also known (Lee, 1980). Cell walls also typically have an amorphous, mucilaginous component which may include uronic acids such as mannuronic or guluronic acid, or sulfated carbohydrates (Lee, 1980). Complex mixtures of monosaccharides, including glucose, galactose, amnose, xylose, fucose, and rhamnose have been found in diatom cell walls (Hecky et al., 1973; Haug and Myklestad, 1976), while dinoflagellate cell walls are 
composed primarily of glucose (Haug et al., 1973), with $\beta(1,3)$ and $\beta(1,4)$ linkages (Nevo and Sharon, 1969).

A number of species of phytoplankton, particularly diatoms (Allan et al., 1972; Smestad et al., 1974; Smestad et al., 1975; Haug and Myklestad, 1976; Percival et al., 1980) and red algae (Kieras et al., 1976; Geresh et al., 1990; Lupescu et al., 1991), excrete carbohydrates. Excreted polysaccharides are commonly branched (Smestad et al., 1974; Smestad et al., 1975; Haug and Myklestad, 1976; Percival et al., 1980), and typically have a heterogeneous composition, which may include mixtures of fucose, galactose, rhamnose, xylose, mannose, and glucose, as well as other components. Charged carbohydrates, such as uronic acids or sulfated sugars, have been found in almost all excreted polysaccharides (Guillard and Hellebust, 1971; Allan et al., 1972; Myklestad et al., 1972; Smestad et al., 1975; Kieras et al., 1976; Percival et al., 1980; Geresh et al., 1990; Lupescu et al., 1991). Not all studies of excreted carbohydrates have included analyses which would detect charged components. One excreted polysaccharide containing amino sugars (Frew et al., 1990) has also been found.

The composition and concentration of both cellular and excreted carbohydrates depends on growth conditions, growth phase, and species. Accumulation of cellular carbohydrates has been associated with nutrient depletion (Myklestad et al., 1972; Vieira and Myklestad, 1986) and with stationary-phase or slowly growing cells (Guillard and Wangersky, 1958; Haug and Myklestad, 1976; Burney et al., 1981). Carbohydrate excretion may also occur during all phases of growth (Vieira and Myklestad, 1986; Myklestad et al., 1989). Reported differences in carbohydrate composition and concentration between species and between cultures therefore are likely to reflect real biological differences. Since the relative difficulty of extraction and characterization of phytoplankton carbohydrates is related to the complexity of the matrix in which they are bound, however, the carbohydrate composition of a particular species may depend on extraction and characterization techniques, as well as growth phase and conditions.

\section{Survey of Phytoplankton Carbohydrates}

\section{Overview of Organisms}

Four species of phytoplankton and one cyanobacterium were cultured to learn more about the types of polysaccharides which are produced in marine environments, and to isolate carbohydrates which would be suitable substrates for bacterial degradation studies. Little information other than monomer composition of hydrolysates and general classes of storage polymers is available for most photosynthetic organisms; by testing a variety of 
extraction techniques and using NMR as a quick screening technique, promising extracts could be selected for further work. In addition, since commercially-available polysaccharides are generally limited to linear homopolymers (usually of glucose), an additional goal was to find a natural source of a more complex polysaccharide. One chlorophyte (green algae), Dunaliella tertiolecta, two prymnesiophytes (coccoliths), Emiliania huxleyi and a Phaeocystis species, one bacillariophyte (diatom), Stephanopyxis palmeri, and one cyanobacterium, Synechococcus WH7335, were cultured for these studies. Synechococcus was ultimately selected for further study, and is described in greater detail.

\section{Cultures}

All cultures were in stationary phase when harvested. The diatom Stephanopyxis palmeri, isolated in Woods Hole by Mark Dennet from Sargasso Sea samples, was grown in filtered Sargasso seawater supplemented with $\mathrm{f} / 2$ vitamins. The cells were grown under continuous light at $20^{\circ} \mathrm{C}$. Stephanopyxis cells are ca. $100 \mu \mathrm{m}$ in length, and tend to clump in long brown bundles or sheaths which resemble a horse's tail.

The Phaeocystis species was grown at $20^{\circ} \mathrm{C}$ under continuous light from a seed culture provided by the Northwest Pacific Culture Collection at the University of British Columbia (species 667; isolated off Surinam by R. Guillard). The HESNW medium (with double vitamins) was made up in filtered seawater from the Coastal Research Center, Woods Hole MA. The medium was autoclaved, sterile-filtered to remove iron precipitates, and aged for at least 48 hours before each culture transfer. Individual Phaeocystis cells are ca. $5 \mu \mathrm{m}$ spheres with two flagella which are lost when Phaeocystis grows in the colonial form. Growth in colonial form is not related to nitrogen or other nutrient limitation (pers. comm., C. Lancelot). Colonial Phaeocystis forms large round and oblong light-brown colored 'balloons' ranging in size from 1 to $8 \mathrm{~mm}$. The other prymnesiophyte in this study, E. huxleyi, was grown in $\mathrm{f} / 2$ medium at $20^{\circ} \mathrm{C}$ under a $12-12$ light-dark cycle.

Cultures of the green algae Dunaliella tertiolecta were grown under continuous light in standard $\mathrm{f} / 2$ medium made up in filtered seawater collected from the clean seawater source at Dyers Dock, Woods Hole MA. Synnechococcus WH7335, a dark green colored cyanobacterium isolated from a coastal area in the Gulf of California by John Waterbury, was grown in S/N medium. The first culture was grown under a 14/10 light/dark cycle at $25^{\circ} \mathrm{C}$, while the second culture was grown at room temperature under ambient daylight and low light at night. The cells grew in a flat, gel-like mat on the bottom of the culture flask, and remained in a cohesive clump until shaken vigorously. 


\section{Excreted Carbohydrates}

Filtered media $(0.2 \mu$ filter) from all cultures except Dunaliella were tested for the presence of carbohydrates using the phenol-sulfuric acid method (Chaplin and Kennedy, 1986). Media from all four species tested were positive for carbohydrates; the Synechoccus medium produced the highest positive response for carbohydrates, followed by Stephanopixis, E. huxleyi, and Phaeocystis.

\section{Extraction of cellular carbohydrates}

Stationary-phase cultures were harvested by centrifuging the medium and removing the pellet. After centrifugation, the medium was filtered through pre-combusted GF/F filters in order to remove the remaining cells. The pellet and filters were stored frozen until analysis. In the cases of E. huxleyi, Phaeocystis, and a second culture of Synechococcus WH7335, the medium was filtered without prior centrifugation, so all extractions of cellular carbohydrates from these species were made from filtered samples.

In all cases, either pre-combusted GF/F filters or the cell pellet (which in the case of Stephanopixis and the first culture of Synechococcus WH7335 was a thick slurry) were ground in $50 \mathrm{~mL} \mathrm{Q}-\mathrm{H}_{2} \mathrm{O}$ with a teflon-bit drill grinder. The aqueous slurry was poured into a separatory funnel, and $50-100 \mathrm{~mL}$ of a 1:1 mixture of methanol and methylene chloride was added. The aqueous mixture was extracted at least three times with fresh organic solvents, or until the organic phase remained colorless upon addition of fresh solvent. The aqueous mixture was usually colorless or light yellow to green after extraction. The organic phases were combined and set aside. The aqueous phase and cellular fragments (and filter fragments, for samples collected from filters) were filtered through a GF/F filter. The fragments and filter were set aside for hot-water extraction, while the aqueous phase was concentrated by rotary-vacuum distillation to remove methanol, and then lyophilized. This fraction is referred to as the cold aqueous extract (CAE) in the following sections. The cellular fragments (including filter, in cases where the fragment cake could not be removed cleanly from the filter) were placed in a roundbottom flask with $50-100 \mathrm{~mL} \mathrm{Q}-\mathrm{H}_{2} \mathrm{O}$ and several boiling chips. After reflux for 20-32 hours, the material was centrifuged or filtered through a GF/F filter to separate the extract from the cell and filter fragments. The hot aqueous extract (HAE) was then lyophilized. All CAE and HAE were tested for the presence of carbohydrates using the phenol-sulfuric acid test (Chaplin and Kennedy, 1986). 
Table 3.1 lists the yields and results of CHN analyses of CAE and HAE of all five organisms. All extracts contained carbohydrates, which demonstrates that harsh conditions are not required to extract a portion of the cellular carbohydrates. The CAE were greater in mass than the HAE, but also contained more salts or non-organic components. All extracts also contained nitrogen; $\mathrm{C} / \mathrm{N}$ ratios of $\sim 4-6$ suggests that protein was a prominant component of the extracts. The chemical shifts and coupling constants of the anomeric protons in the NMR spectra of the extracts indicated that $\alpha$-linked carbohydrates were much more common than $\beta$-linked carbohydrates in both types of extracts. NMR spectra of all extracts with the exception of the Synechococcus CAE showed the presence of large quantities of non-carbohydrate material; further work would be required to purify the carbohydrates.

\section{Hot Aqueous Extracts}

With the exception of the HAE from Dunaliella, the HAE generally were less soluble and had fewer well-resolved NMR resonances than the CAE. NMR spectra were typically dominated by poorly resolved resonances and broad, irregular humps. For each extract, anomeric resonances were distinguishable, but extensive purification would be required to determine further structural information about the carbohydrates in most of the HAE.

The spectrum of Stephanopixis shows possible anomeric resonances at $\delta=5.05$, 5.2, 5.28, 5.5, and 5.55 (Fig. 3.1a), but otherwise has few distinguishing features which are consistent with NMR spectra of carbohydrates. Low humps at $\delta=5.45$ and 5.5 in the Phaeocystis spectrum might correspond to bound $\alpha$-anomers (Fig. 3.1b). A portion of the extract was hydrolyzed for 5 hours in $4 \mathrm{M} \mathrm{TFA}$ at $100^{\circ} \mathrm{C}$; the spectrum of the hydrolyzed hot aqueous extract shows several distinct anomeric resonances (Fig. 3.1c). The low humps at $\delta=5.45$ and 5.5 are no longer visible, but a series of resonances between $\delta=5.12$ 5.3 , consistent with free monosaccharides, are apparent, as is a distinct $\alpha$-anomer at $\delta=4.95$. A doublet which may be the corresponding free $\beta$ anomer is evident at $\delta=4.4$. The HAE of E. huxleyi is similar to that of Phaeocystis, in that low humps at $\delta=5.3,5.4$, and 5.5 could correspond to bound $\alpha$-anomers (Fig. 3.1d). The HAE of Synechococcus also shows a possible bound $\alpha$-anomer at $\delta=5.45$ (Fig. 3.1e). Hydrolysis of a portion of the Synechococcus HAE for three hours in $4 \mathrm{M}$ TFA at $100^{\circ} \mathrm{C}$ sharpened many of the resonances (Fig. 3.1f). In the anomeric region, a low resonance at $\delta=5.45$ is still visible, and a number of sharp doublets at $\delta=5.25$ and 4.95 , probably corresponding to free $\alpha$ anomers, are apparent. The appearance of the spectrum in the region from $\delta=3.6-4.0$ also sharpened considerably, and resembles the proton spectrum of a polysaccharide. 
culture

Dunaliella tertiolecta

Emiliani huxleyi culture volume

$10 \mathrm{~L}$

extracted from filters: not weighed

CAE: $228.64 \mathrm{mg}$

(extr. 1-1)*

(extr. 1-2)*

(extr. 2)*

HAE: $26.65 \mathrm{mg}$

extracted from filters: $42.20 \mathrm{mg} * *$
C:H:N

4.63: 0.75: 1.15

2.73: $0.53: 1.18$

15.05: 3.64: 1.85

38.66: $4.14: 9.38$

Phaeocystis sp. (8/92) $\quad$ 1.5 L $\quad$ CAE: $7.90 \mathrm{mg} \quad$ 14.91:1.49: 3.40 HAE: 26.75 mg** $3.94: 0.71: 0.74$

Phaeocystis sp. (12/92) $\quad 1.5 \mathrm{~L} \quad$ CAE: $55.95 \mathrm{mg}$ (Note that the difference in yield between the two Phaeocystis cultures is due to more vigorous growth and estimated higher cell density in the 12/92 culture)

Stephanopixis palmeri

Synechococcus WH7335 $(10 / 92)$

Synechococcus WH7335 (1/93)
$18 \mathrm{~L}$

$0.8 \mathrm{~L}$

$0.8 \mathrm{~L}$
CAE: $134.80 \mathrm{mg} * * *$ HAE: $19.60 \mathrm{mg}$
5.03: 0.72: 1.24 


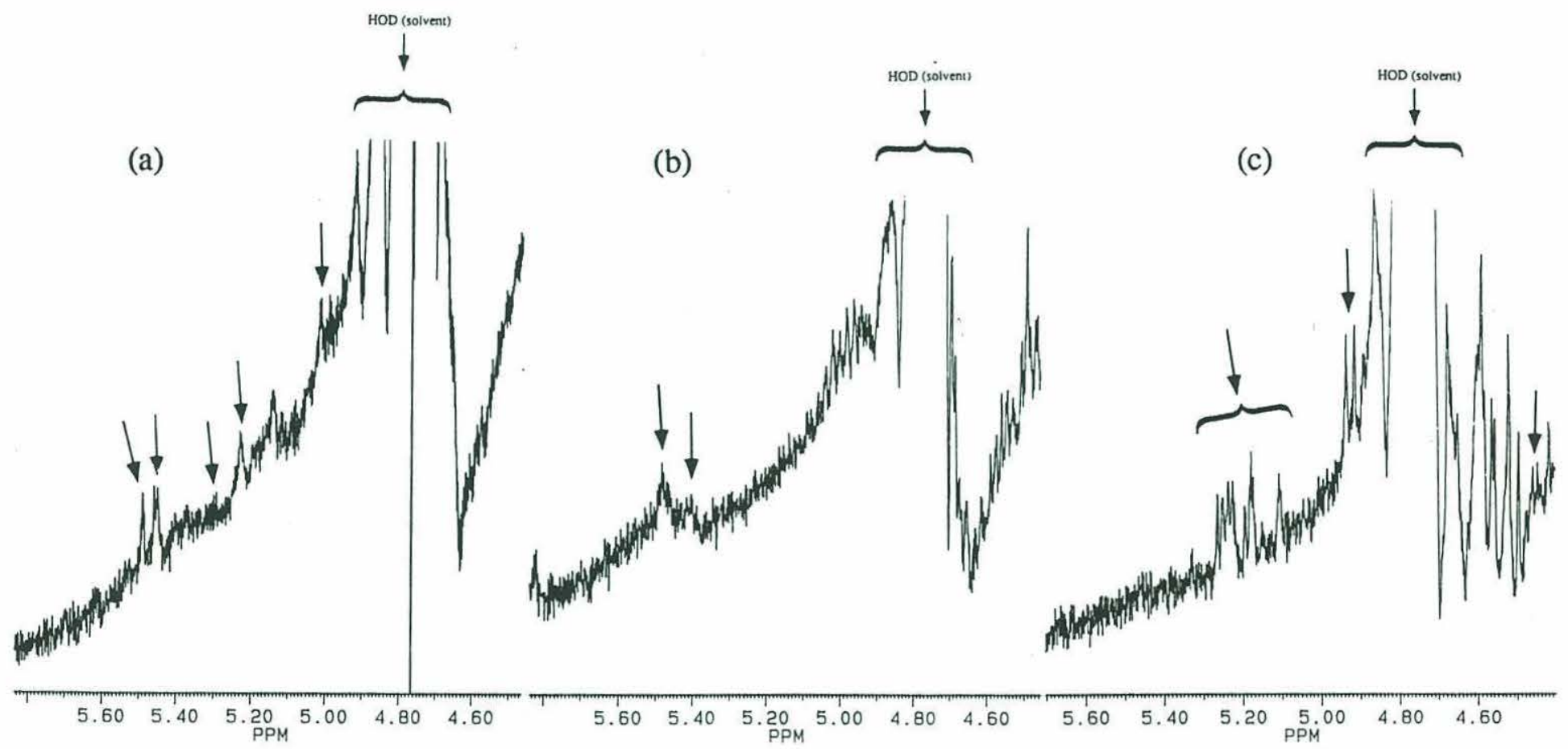

Fig. 3.1a-c

Anomeric regions of Stephanopixis (a), Phaeocystis (b), and Phaeocystis hydrolyzed (c) hot aqueous extracts. Arrows mark anomeric proton resonances. 


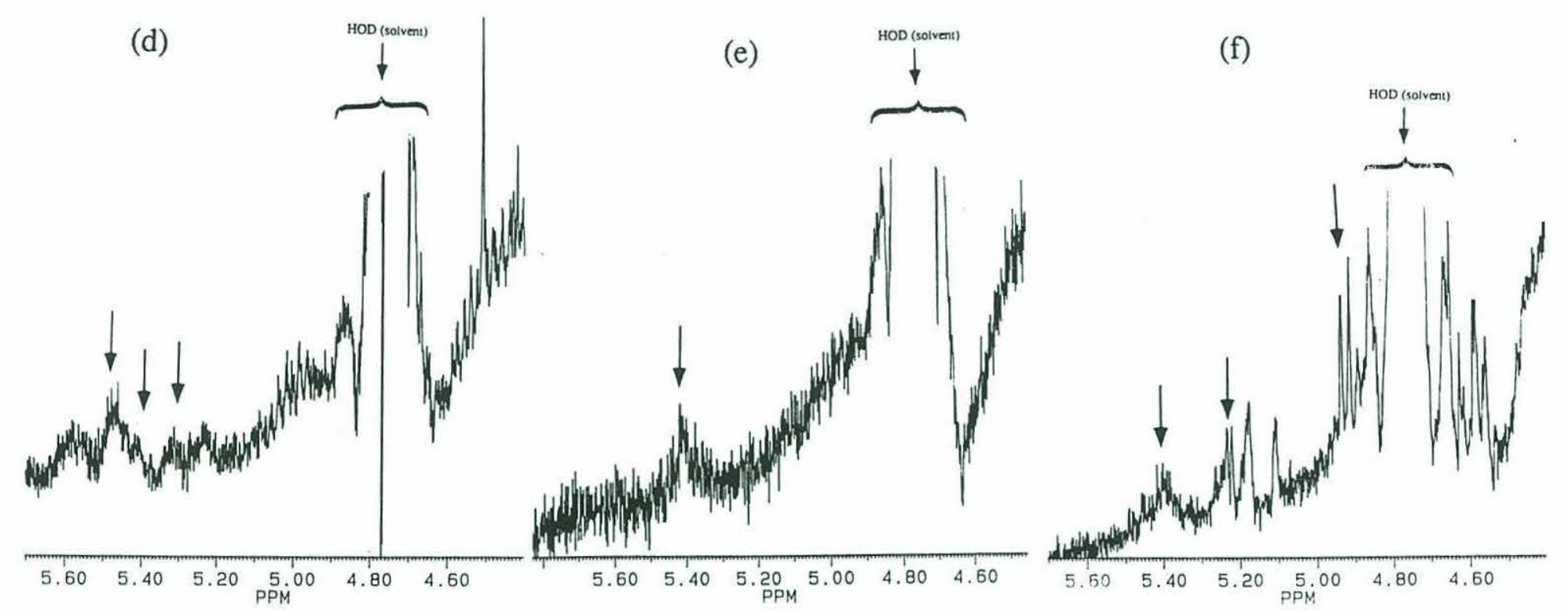

Fig. 3.1d-f

Anomeric regions of E. huxleyi (d), Synechococcus WH7335 (e), and Synechococcus WH7335 hydrolyzed (f) hot aqueous extracts. Arrows mark anomeric proton resonances. 
These hydrolysis conditions are sufficient to hydrolyze maltoheptaose completely to glucose, so if some of the Synechococcus material is still in polymeric form, it must be relatively resistant to acid hydrolysis.

The HAE of Dunaliella tertiolecta, in contrast to those of other organisms, was very high in carbon, relatively rich in carbohydrates, and yielded an NMR spectrum with a readily identifiable structure. A search of the literature revealed only two previous reports on the carbohydrates of Dunaliella tertiolecta. A solid-state ${ }^{13} \mathrm{C}-\mathrm{NMR}$ analysis of whole organisms and humic-like material from Dunaliella cultures showed that carbons with chemical shifts consistent with those of neutral carbohydrates were a major component of Dunaliella extracts (Zelibor et al., 1988). The monosaccharide composition of Dunaliella principally consists of glucose ( $85.3 \%$ of total carbohydrates), with smaller concentrations of rhamnose $(5.5 \%)$, mannose $(4.5 \%)$, ribose $(2.0 \%)$, galactose $(1.1 \%)$, xylose $(1.0 \%)$, and arabinose $(0.65 \%)$. Total carbohydrates were a surprisingly low $12.2 \%$ of cell dry weight; lipids and protein were $15 \%$ and $20 \%$ of total cell weight, respectively (Brown, 1991). Total carbohydrates of Dunaliella salina, in contrast, comprised $31.6 \%$ of cell dry weight, with lipids and proteins making up $6.4 \%$ and $57 \%$ of cell dry weight, respectively. Glucose was also the principal monosaccharide of D. salina ( $54.5 \%$ of total carbohydrates), with galactose (37.3\%) and ribose (5.4\%) as well as uronic acids as the remainder of the carbohydrates (Parsons et al., 1961).

The full spectrum of the Dunaliella HAE (Fig. 3.2) shows a prominant anomeric resonance at $\delta=5.4$, and relatively few non-carbohydrate resonances (the region $\delta=0.8$ 2.2). (Note that saturated solutions of the Dunaliella HAE showed a greater predominance of non-carbohydrate resonanaces in this region. These resonances could be due to protein components, which would also account for the $\mathrm{N}$ in the HAE.) All of the resonances in the region from $\delta=3.2-5.4$ (Fig. 3.3a) resemble those of a high molecular weight polysaccharide. The single major $\alpha$-anomer at $\delta=5.4$ is consistent with an $\alpha(1,4)$ linkage. Since there are no other anomeric resonances, the Dunaliella HAE probably contains a homopolysaccharide with one linkage type and no branches. Homopolysaccharides are often stored as energy reserves in phytoplankton; the relatively large amount of the Dunaliella HAE (in terms of mass and carbon content of the extract) suggests that it is a significant proportion of total cell mass, which is also consistent with a role as an energy reserve. Green algae as a class are reported to accumulate starch (Lee, 1980), inulin, and possibly laminarin (Painter, 1983) as energy reserves. The appearance of the spectrum is not consistant with inulin, a fructose-glucose polysaccharide with $\beta$ linkages, since inulin would have several prominant anomeric resonances corresponding to glucose and fructose. In addition, $\beta$-anomers are generally found upfield of the HOD (solvent) resonance at 
93

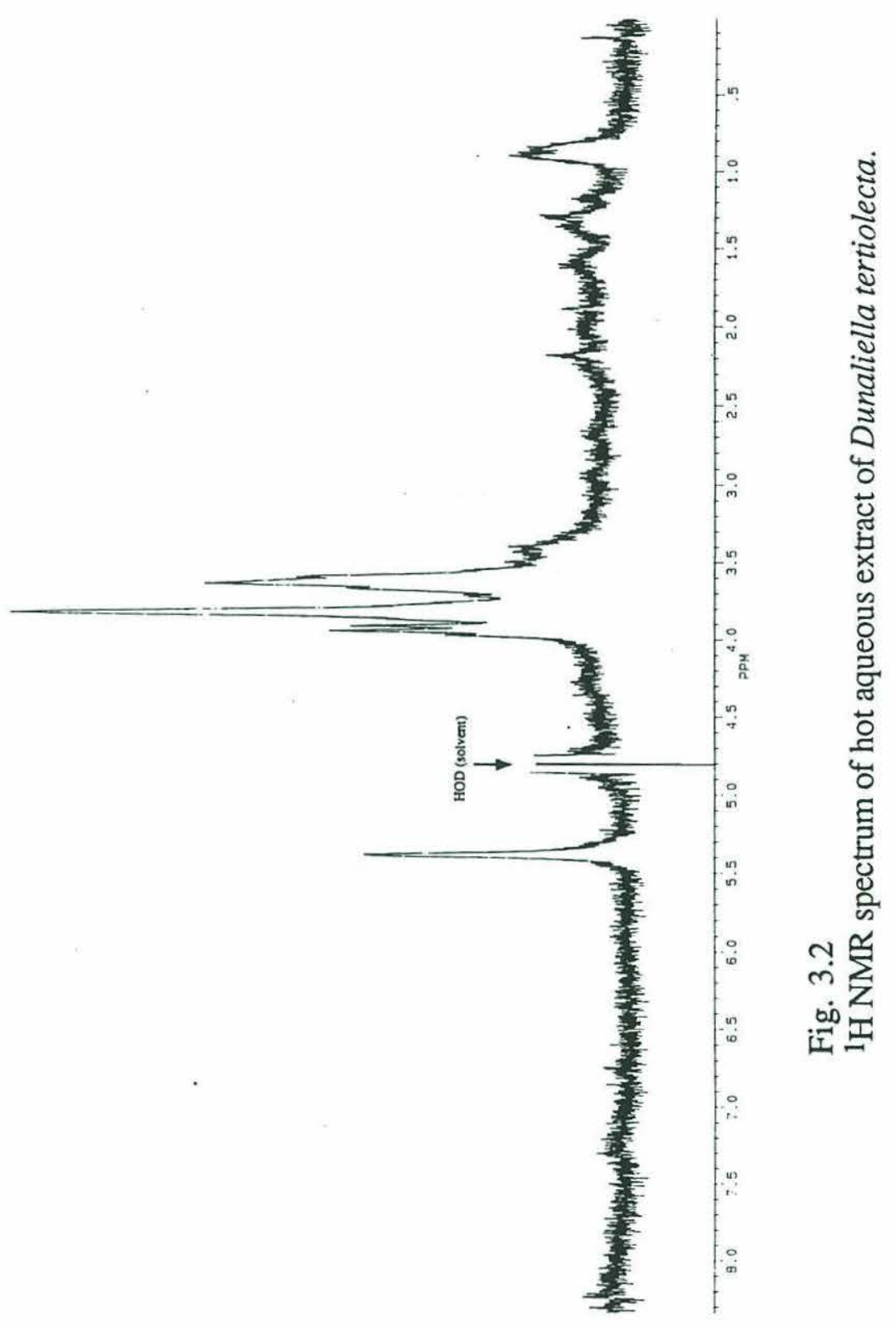


(a)

extract of Dunaliella tertiolecta

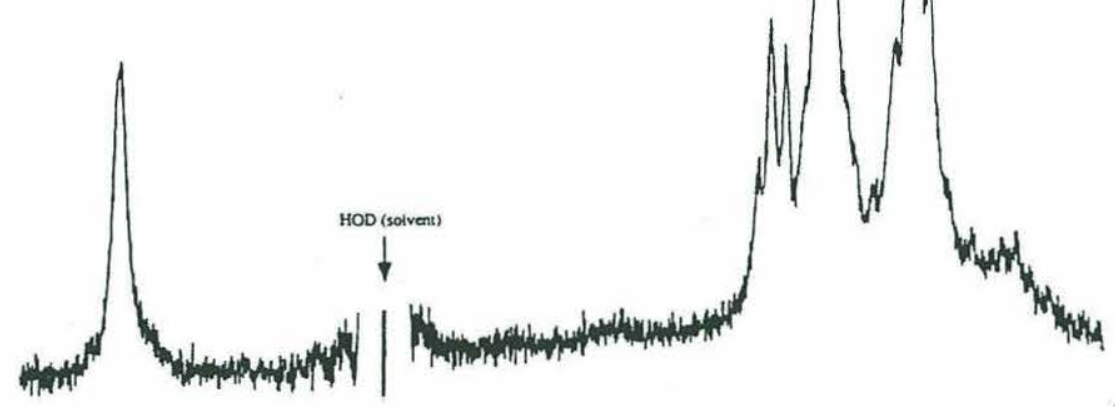

(b)

amylose $(\alpha(1,4)$ glucose polymer)

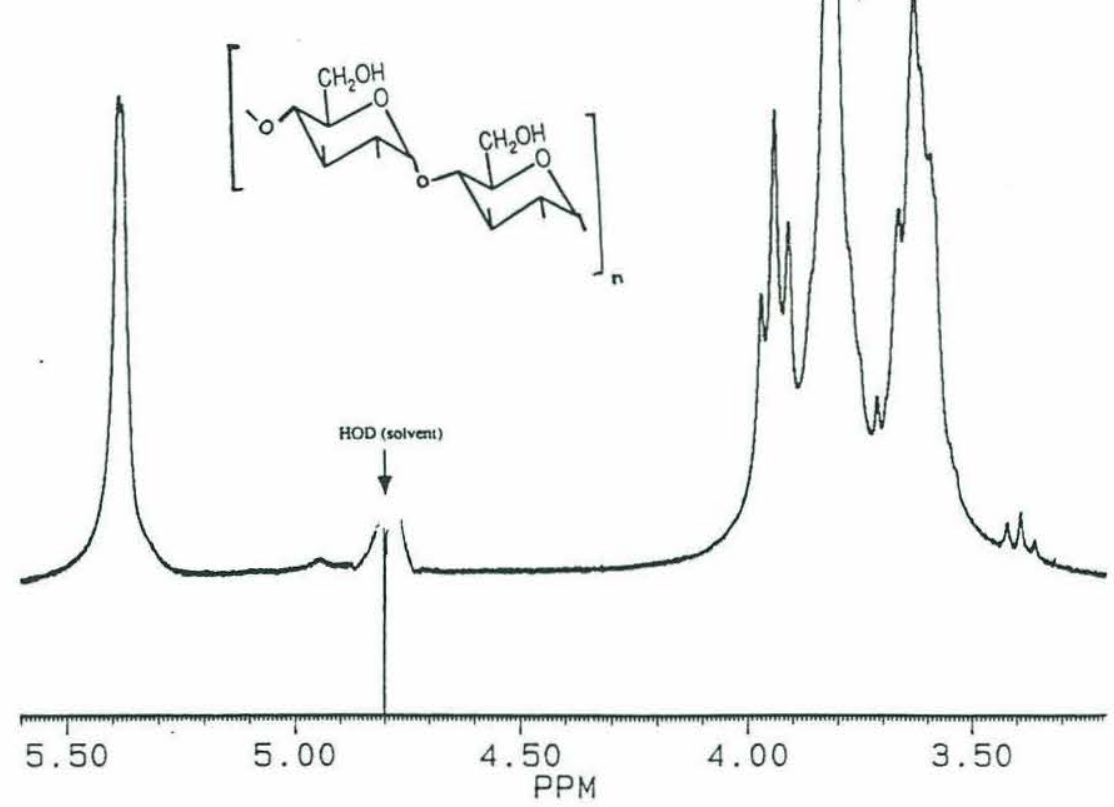

Fig. 3.3

Partial ${ }^{1} \mathrm{H}$ NMR spectrum of Dunaliella tertiolecta hot aqueous extract (a) and ${ }^{1} \mathrm{H}$ NMR spectrum of amylose standard (b). 
$\delta=4.76$. The $\beta$-anomer of laminarin (a $\beta(1,3)$-linked glucose polysaccharide) is one of the few exceptions to this general rule, since it resonates close to $\delta=4.9$. As a comparison with Fig. 2.17 shows, however, the Dunaliella polysaccharide does not resemble laminarin. Starch is a combination of amylose, a linear $\alpha(1,4)$ glucose polysaccharide, and amylopectin, an $\alpha(1,4)$ glucose polysaccharide with $\alpha(1,6)$ branches. A spectrum of an amylose standard, which closely matches the Dunaliella polysaccharide in Fig. 3.3a, is shown in Fig. 3.3b. These spectra suggest that Dunaliella tertiolecta contains amylose, the unbranched glucose polysaccharide, as a storage product.

The Dunaliella HAE was the only case in which the hot-water extract of an organism yielded more soluble 'carbohydrate-like material' (i.e., recognizable carbohydrate-like features in the NMR spectrum) than the cold-water extract. This may be due to the fact that storage products of chlorophytes are found as grains inside the chloroplasts, while for prymnesiophytes and bacillariophytes, storage products are located outside of the chloroplast (Lee, 1980). Longer and more vigorous extraction procedures (reflux for 20 hours) may be required to solubilize storage products which are inside the chloroplast. The difference in relative carbohydrate content may also simply be a function of solubility, since amylose is relatively insoluble in cold water (see Chapter 2).

The generally higher carbon content of the HAE compared to the CAE may indicate that the HAE typically contain high quantities of carbohydrates which are relatively insoluble, either because of molecular weight or specific chemical structure. The chemical shift and appearance (poorly-resolved J-coupling) of the proton resonances observed in the HAE is consistent with those of polysaccharides. The changes in the NMR spectra of Phaeocystis and Synechocccus HAE after hydrolysis in 4M TFA also suggest that the material in the HAE is primarily high molecular weight. Gel permeation chromatography of the HAE would provide an estimate of molecular weight and could be used to separate the components of the HAE.

\section{Cold Aqueous Extracts}

All of the CAE were very soluble, and contained prominent anomeric resonances. Their low carbon content suggested that they contain high quantities of non-organic compounds as well. The NMR spectra in general were quite well-resolved, with well-defined resonances and J-coupling and fewer unresolved humps than seen in the HAE.

The CAE of Dunaliella (Fig. 3.4a) shows a well-resolved anomeric resonance at $\delta=5.37$, probably from a bound $\alpha$-anomer, since the free $\alpha$-anomers of most neutral carbohydrates resonate in the region $\delta=5.1-5.3$. The majority of the resonances (in terms of 


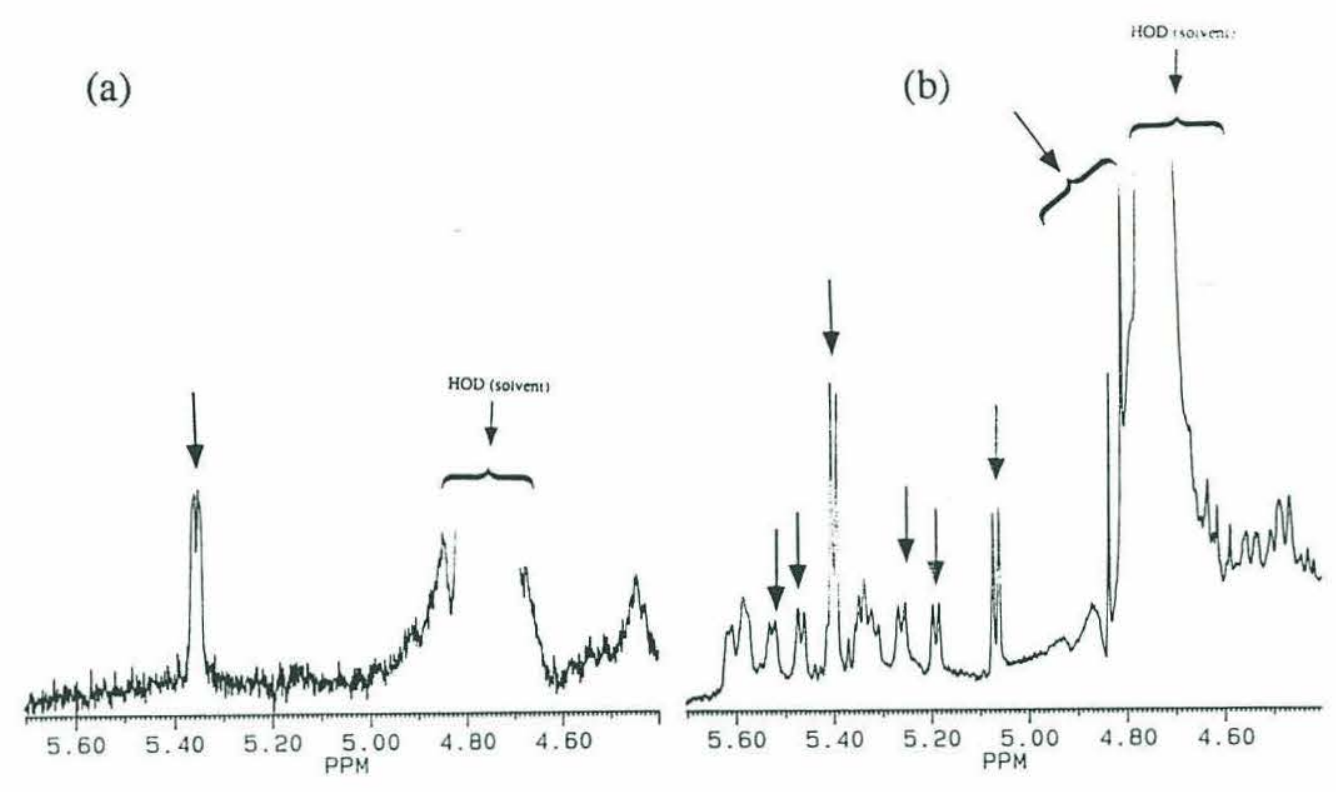

(c)

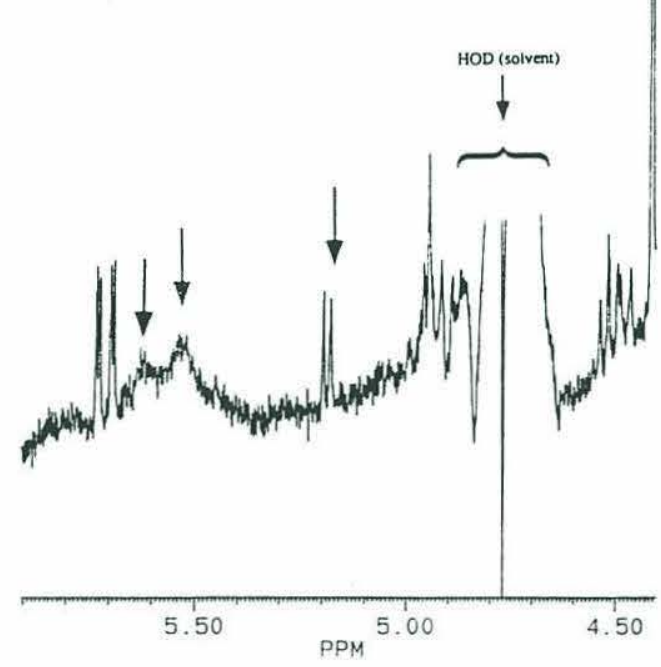

(d)

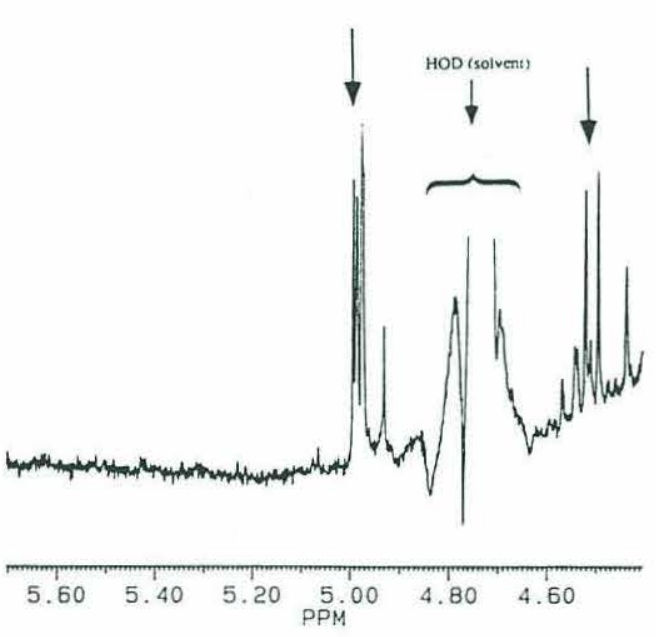

Fig. 3.4a-d

Anomeric regions of Dunaliella (a)Stephanopixis (b), Phaeocystis (c), and E. huxleyi (d) cold aqueous extracts. Arrows mark anomeric proton resonances. 
both area and number) are in the region $\delta=3.0-4.5$, which is the region in which most carbohydrate resonances are found. A series of doublets and quartets (nucleic acids?) were observed in the region $\delta=0.9-3.3$.

The anomeric region of the Stephanopixis CAE (Fig. 3.4b) shows several diagnostic resonances. The doublet at $\delta=4.82$ has the chemical shift and J-coupling characteristic of a $\beta(1,3)$ linked polysaccharide such as laminarin (see Fig. 2.17). Laminarin is a known storage product of diatoms (Lee, 1980; Painter, 1983), although this is the first suggestion of its presence in Stephanopixis palmeri. Laminarin is very soluble in $\mathrm{H}_{2} \mathrm{O}$; its presence in the CAE supports the previous suggestion that relative solubility (and perhaps location outside the chloroplast) may determine the type of procedure required to extract cellular carbohydrates. Clearly, mild extraction procedures can be used to obtain highly soluble polysaccharides.

The presence of several other types of carbohydrates is indicated by the prominent doublets at $\delta=5.08$ and 5.40, and the smaller resonances at $\delta=5.20,5.28,5.49$, and 5.52. The chemical shifts and J-couplings are indicative of $\alpha$-anomers. The less well-resolved peaks between $\delta=5.32-5.4$ and $\delta=5.57-5.62$ may likewise be $\alpha$-anomers. Several of the small resonances in the region $\delta=4.5-4.68$ could be $\beta$-anomers, although their low intensity makes positive identification difficult. The majority of the resonances in the cold aqueous extract fall in the region between $\delta=3.0$ and 4.4 , which is consistent with carbohydratecontaining material. A cluster of poorly-resolved, low intensity resonances between $\delta=7.4$ and 7.65 could be due to aromatic protons, while a number of aliphatic resonances, including well resolved doublets, are in the region $\delta=0.9-2.9$.

The anomeric region of the Phaeocystis CAE (Fig. 3.4c) is relatively noisy, but a few anomeric resonances are clearly visible, such as the distinct doublet at $\delta=5.2$. The chemical shift and the depth of the splitting suggest that this anomer may correspond to a free $\alpha$-anomer or the $\alpha$-anomer of a low molecular-weight carbohydrate. A number of sharp resonances in the region $\delta=4.45-4.55$ may likewise correspond to free $\beta$-anomers. Several other possible anomeric resonances are visible in the region $\delta=5.5$ and 5.6 ; these likely correspond to bound $\alpha$-anomers. The water-suppression in the region $\delta=4.9$ is noisy, so it is difficult to determine if any of the peaks between $\delta=4.45-4.85$ are due to carbohydrate proton resonances. The pair of doublets centered around $\delta=5.7$ probably represent a doublet of doublets, not an anomeric resonance. The rest of the spectrum shows a number of distinct aromatic-type resonances in the regions between $\delta=6.0-6.3$, and 7.78.1. A number of poorly-resolved resonances, some of which resemble high-molecular weight carbohydrate resonances, dominate the region from $\delta=3.5-4.2$, while the region 
from $\delta=0.9$ to 3.5 contains poorly-resolved humps as well as a few sharp singlets and doublets.

The spectrum of the E. huxleyi CAE (Fig. 3.4d) shows several prominent resonances from $\delta=4.95-5.05$. The strong doublet at $\delta=4.55$ could be a $\beta$ anomeric resonance. Carbohydrate-like resonances are found in the region $\delta=3.5-4.0$, while a series of well-resolved doublets are found in the aromatic region $(\delta=5.8-7.6)$, and the upfield portion of the spectrum from $\delta=0.9$ to 3.0 is dominated by sharp singlets and doublets.

The chemical shifts and J-couplings of the anomeric protons in the CAE were generally consistent with mono- or oligosaccharides. The J-couplings of the bound anomers were well-resolved, which is typical of oligosaccharides but not polysaccharides (see Figs. 2.13, 2.14, and 2.15, for example). Some carbohydrates in the HAE and CAE may differ only in molecular weight, since the partitioning of aqueous-extractable carbohydrates in the HAE and CAE based partially on solubility. For certain cellular components such as energy reserves, phytoplankton probably synthesize a continuum of carbohydrate sizes, so certain types of carbohydrates differing only in molecular weight might be found in both fractions. One example might be the Dunaliella $\mathrm{HAE}$ and CAE, which both had prominant $\alpha$-anomers at $\delta=5.4$. This chemical shift corresponds to glucose $\alpha(1,4)$-linked anomeric protons. Monosaccharide analysis of the Dunaliella HAE is all that is needed to show that this extract contains amylose; the CAE might contain maltooligosaccharides, which (unlike amylose) are soluble in cold water.

\section{The Carbohydrate of Synechococcus WH7335}

The cold aqueous extract of Synechococcus was greatest in mass and richest in carbohydrates of all of the extracts tested. The goal of this work was to obtain carbohydrates for use in bacterial degradation experiments (Chapter 5), and to further characterize the carbohydrates of Synechococcus WH7335. The following section will present a brief overview of previous work on the carbohydrates of cyanobacteria, followed by the characterization of the carbohydrates of Synechococcus WH7335.

\section{Carbohydrates of Cyanobacteria}

Cyanobacteria contain carbohydrates in the cell membrane and as energy reserves, and can also excrete carbohydrates. Carbohydrates can be a significant fraction of total organic matter; in a Nostoc sp. for example, carbohydrates comprised 23-30\% of total cellular matter (Mehta and Vaidya, 1978). The cell membrane of cyanobacteria is high in 
carbohydrates, and shares characteristics of cell membranes of both gram-negative and gram-positive bacteria. Like gram-negative bacteria, cyanobacteria have an outer membrane surrounding the cytoplasmic membrane. The cell wall, which may be up to $50 \%$ of total dry cell mass, is composed principally of murein, a peptidoglycan (Lee, 1980). The peptidoglycan layer of cyanobacterial cell walls is similar in thickness to that of grampositive bacteria, and also resembles gram-positive bacteria in degree of cross-linking, and in the presence of covalently bound polysaccharides (Weckesser and Jurgens, 1988). A polysaccharide of $25-30 \mathrm{kD}$, for example, was found to be covalently linked to the peptidoglycan of Synechocystis sp. strain PCC6714 (Jurgens and Weckesser, 1986). The peptidoglycan components of cyanobacterial cell walls can include glucose amine, galactose amine, and mannose amine. Lipopolysaccharides, composed of mannose, galactose, and glucose, as well as O-methyl sugars such as O-Me-xylose, -mannose, -arabinose, -fucose, and -rhamnose, may also be cell wall components (Drews and Weckesser, 1982). Uronic acids have also been found in cell walls (Schmidt, 1980; Bertocchi et al., 1990).

The composition of carbohydrate energy reserves is much simpler than cell wall composition. Granules known as " $\alpha$-granules" accumulate in the space between the thylakoids in actively photosynthesizing cells (Lee, 1980). These granules are made of 9$26 \alpha(1,4)$-linked glucose units, with $\alpha(1,6)$ branches. The polysaccharide can comprise $10-30 \%$ of total cell dry weight (Shivley, 1988).

Most cyanobacteria have an extracellular mucilage layer (often referred to as the "sheath", "capsule", or "slime layer") which surrounds the cell wall (Lee, 1980). The sheath can be up to $1 \mu \mathrm{m}$ thick. Some cyanobacteria synthesize a fibrous external layer which can envelope several cells, while others excrete a slime/mucilage which may partly dissolve and no longer be associated with the cells; these slimy layers do not have well defined structure (Drews and Weckesser, 1982). Sheath carbohydrates may include Omethyl sugars (Bertocchi et al., 1990).

Some species of cyanobacteria have been shown to excrete large quantities of exopolysaccharides; extracellular polysaccharides were 14-18\% of total biomass, for example, in cultures of a species of Nostoc (Mehta and Vaidya, 1978). Cyanobacterial exopolysaccharides are quite complex in composition; xylose, arabinose, ribose, glucose, galactose, mannose, fucose, rhamnose, and glucuronic acid have all been reported (Drews and Weckesser, 1982). Sulfate groups have also been found in some exopolysaccharides (Bertocchi et al., 1990). No compositional information beyond monosaccharide composition has been reported for exopolysaccharides. 


\section{Extraction of Synechococcus WH7335}

Two cultures of Synechococcus WH7335 were extracted for this study. The first culture was harvested by initially centrifuging the medium to concentrate a viscous cellular pellet which was removed from the flask by pipet; the medium was then filtered through precombusted GF/F filters to harvest the cells which had not settled during centrifugation. The cell pellet and the filters were stored frozen, and extracted separately. Both yielded the same fractions when subjected to gel chromatography, but salt concentration appeared to be significant particularly in the cell pellet fractions (note that the mass of the cold water extract from the first culture was nearly double that of the second culture, Table 3.1). The second culture, therefore, was harvested by filtration without prior centrifugation. Because of the high viscosity of the medium, only ca. $40 \mathrm{~mL}$ of medium could be filtered through each $47 \mathrm{~mm} \mathrm{GF/F} \mathrm{filter.} \mathrm{Each} \mathrm{filter} \mathrm{was} \mathrm{then} \mathrm{rinsed} \mathrm{with} \mathrm{a} \mathrm{few} \mathrm{mL}$ of $\mathrm{Q}-\mathrm{H}_{2} \mathrm{O}$ in order to remove as much salt as possible. The filters were frozen, and then extracted as previously described.

The spectrum of the CAE (Fig. 3.5) had relatively few resonances which are definitely attributable to non-carbohydrate protons (the region from $\delta=0.9-2.8$ ). An expansion of the region between $\delta=5.85$ and 5.7 (inset, Fig. 3.5) shows six distinct $\alpha$ anomeric resonances at $\delta=4.87,5.10,5.12,5.17,5.20$, and 5.39. The sharpness of the resonances suggests that at least the major component of the $\mathrm{CAE}$ is an oligosaccharide, rather than a polysaccharide (see Figs. 2.15, 2.17, and 2.18 for comparison).

\section{Fractionation of the Cold Aqueous Extract}

The lyophilized CAE was dissolved in $\mathrm{Q}-\mathrm{H}_{2} \mathrm{O}$, and fractionated by gel permeation chromatography. The first culture was initially fractionated on a Sephadex G-25 column (100-300 $\mu$ mesh, nominal exclusion on a dextran basis: 5000 daltons), $1.5 \times 33.8 \mathrm{~cm}$., with a void volume of $27.3 \mathrm{~mL}$ (corresponding to $24.8 \mathrm{~min}$.) as calibrated with pullulan, and a total volume of $59.8 \mathrm{~mL}$. The mobile phase was $\mathrm{Q}-\mathrm{H}_{2} \mathrm{O}$, pumped at $1.1 \mathrm{~mL} / \mathrm{min}$ with a peristaltic pump (Pharmacia P-1). Carbohydrates were detected with a refractive index detector (Showdex RI-71, detection limit ca. $5 \mu \mathrm{g}$ carbohydrate), and data were acquired with a Hewlett-Packard 3396 II integrator. Tests showed that better separation was achieved on a Sephadex G-50 column (nominal exclusion: 10,000 daltons, 100-300 $\mu$ mesh), which was used to fractionate the extract from the second culture. The G-50 column 


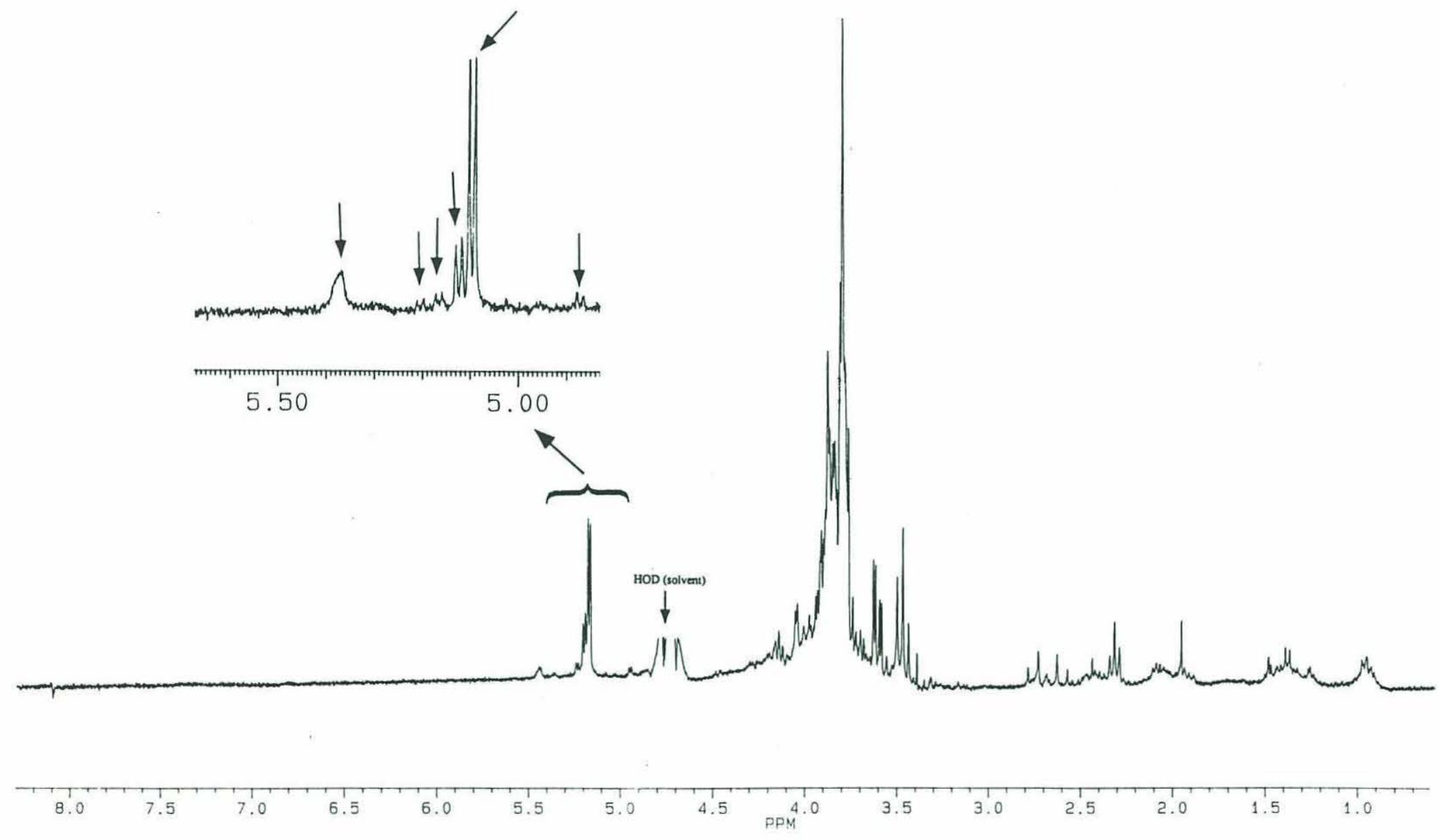

Fig. 3.5

${ }^{1} \mathrm{H}$ NMR spectrum of Synechococcus WH7335 cold aqueous extract. Inset shows expansion of anomeric region $(\delta=4.5-5.7)$. 
was $1.5 \times 28.5 \mathrm{~cm}$, with a void volume of $19.5 \mathrm{~mL}$ (as calibrated with pullulan, MW 200,000 daltons) and a total volume of $49.6 \mathrm{~mL}$.

The lyophilized CAE was dissolved in $8 \mathrm{~mL} \mathrm{Q}-\mathrm{H}_{2} \mathrm{O}$, and filtered through a $0.2 \mu$ syringe filter to remove insoluble particles. One $\mathrm{mL}$ of the solution $(1.1 \mathrm{~mL}$ total volume, with rinse) was injected through a three-way injection port (Alltech). Figure 3.6 shows a typical integrator trace: the extract was collected in five portions, F1 (15-22 minutes), F2 (22-31 minutes), F3 (31-48 minutes), F4 (48-64 minutes), and F5 (64-105 minutes). Similar fractions were pooled and lyophilized, then dissolved in $0.5 \mathrm{~mL} \mathrm{D}_{2} \mathrm{O}$ and examined by NMR. Recovery of the CAE from the column was complete; of the $147.5 \mathrm{mg}$ original extract, $149.4 \mathrm{mg}$ was recovered: $3.1 \mathrm{mg}$ was recovered as F1, approx. $0.5 \mathrm{mg}$ as F2, $144 \mathrm{mg}$ as F3, and $1.8 \mathrm{mg}$ as F4. (Fraction 5 yielded only a few grains of material). The slight discrepency in mass is probably due to residual water in F3, which tended to lyophilize as a waxy mass.

CHN analysis (Table 3.1) of a small amount of F3 which remained after the polysaccharide degradation experiments (Chapter 5 ) showed that this fraction still contained high quantities of nitrogen. While some of the nitrogen probably comes from protein (which would account for the high-field proton resonances $(\delta=1.0-2.8)$ observed in ${ }^{1} \mathrm{H}$ NMR the spectrum (Fig. 3.5, for example)), the lack of observable ${ }^{13} \mathrm{C}$ resonances in the region downfield of $\delta=100$ (explained further in the next section) precludes the presence of a large quantity of proteins. (Small quantities of amino acids or proteins would not be observable in the ${ }^{13} \mathrm{C}$ spectrum.). Some of the nitrogen may occur in the form of residual salts which co-eluted with F3.

A comparison of NMR spectra of the fractions showed that both the first and second Synechococcus cultures produced the same material. In the NMR investigations below, all fractions (with the exception of Fraction 2) were from the second culture, since the high concentration of salts (especially in Fraction 3 ) adversely affects the spectra. The second culture produced less of Fraction 2 than the first culture (see discussion below), so the material from the first culture was used for the NMR and compositional investigations of Fraction 2.

\section{NMR Analysis of Fraction 1-Fraction 5}

Fraction 1 corresponded to the void volume of the column $(>10 \mathrm{kD})$; although the spectrum showed several possible anomeric peaks at $\delta=5.0,5.1,5.25,5.3$, and 5.4 , at least half of the proton resonances corresponded to non-carbohydrate material (broad peaks between $\delta=0.9$ and 2.6 ), so this fraction was not investigated further. 


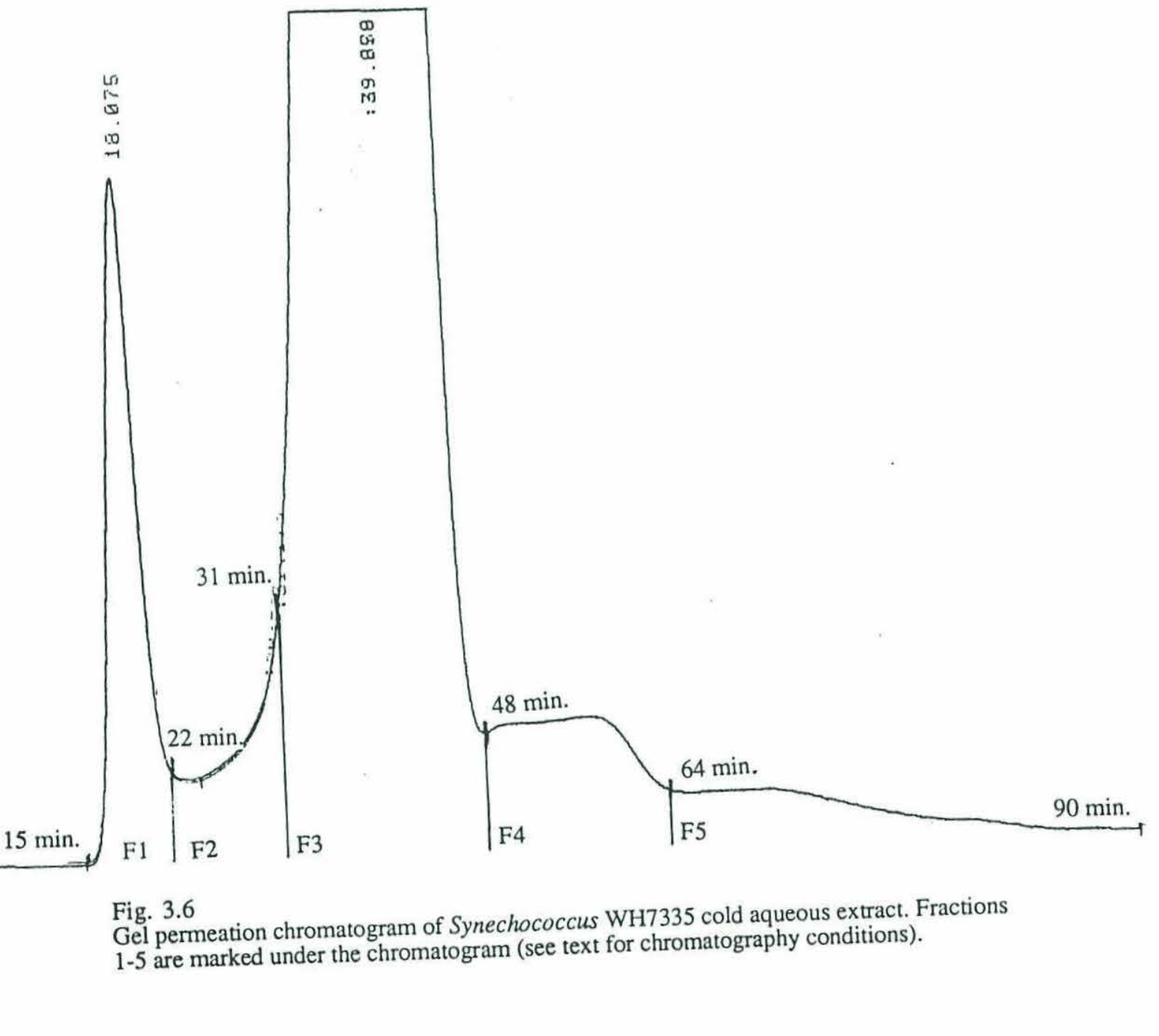

Fig. 3.6

Gel permeation chromatogram of Synechococ ext for chromatography conditions). 
Fractions 2 and 3 will be discussed at length below. Fraction 4 (Fig. 3.7) corresponded to very low molecular weight material; the proton spectrum showed two distinct $\alpha$-anomers, a deeply-split (free?) resonance at $\delta=5.1$, and a broader (bound?) resonance at $\delta=4.95$, which may correspond to an $\alpha(1,6)$ linkage in a glucose oligosaccharide. The resonances between $\delta=3.1$ and 4.0 were consistant with a small oligosaccharide. After lyophilization, Fraction 5 yielded only a few grains of white material (probably salt), which did not produce a signal in an NMR spectrum acquired for several hours.

\section{Fraction 2}

The elution profile of F2 on the Sephadex G-25 column corresponds to a molecular weight of 5-6 kD (based on a laminarin standard). The spectrum of F2 (Fig. 3.8) shows a distinct anomeric resonance at $\delta=4.95$, a low resonace at $\delta=5.1$ which may correspond to a free $\alpha$ anomer, and several overlapping resonances centered around $\delta=5.35$. The overlapping resonances suggest that $\mathrm{F} 2$ is a branched- or heteropolysaccharide. If $\mathrm{F} 2$ were a mixture of linear polysaccharides, the resolution of the anomers at $\delta=5.35$ should have been as high as the anomer at $\delta=4.95$. The broad resonance at $\delta=5.35$, and the behavior of F2 upon rechromatography (explained below) provided strong evidence that $\mathrm{F} 2$ is a branched-or heteropolysaccharide. The resonances in the region $\delta=3.3-4.1$ show the general appearance of a polysaccharide. A proton COSY spectrum of F2 (not shown) had generally low resolution, which is also consistant with $\mathrm{F} 2$ being a high moleuclar weight polysaccharide. The spectrum does distinctly show a cluster of anomeric resonances and their crosspeaks with \#2 protons (Fig. 3.9); the \#2 protons are all in the region $\delta=3.52-3.59$.

A sample of F2 was analyzed for carbohydrate composition at the Complex Carbohydrate Research Center (University of Georgia, Athens GA). The sample was converted to TMS methylglycosides, which were analyzed by gas chromatography-mass spectrometry. Glucose was the only carbohydrate detected in the sample, so F2 is not a heteropolysaccharide. Based on the NMR spectrum, which clearly showed that F2 either was a branched- or heteropolysaccharide, F2 must be a branched glucan. The behavior of F2 on the G-50 column further supports this hypothesis, since when F2 was rechromatographed on the G-50 column, three overlapping subfractions with retention times of 18,34 , and 42 minutes were detected. A retention time of 42 minutes would nominally correspond to an oligosaccharide of 3-7 sugar units, which is inconsistent with the NMR spectrum. The spectra of all three subfractions were virtually the same, in spite of the difference in their retention times. The three subfractions probably differ in degree of branching, which would account for the differences in their chromatographic behavior. 


\section{5}

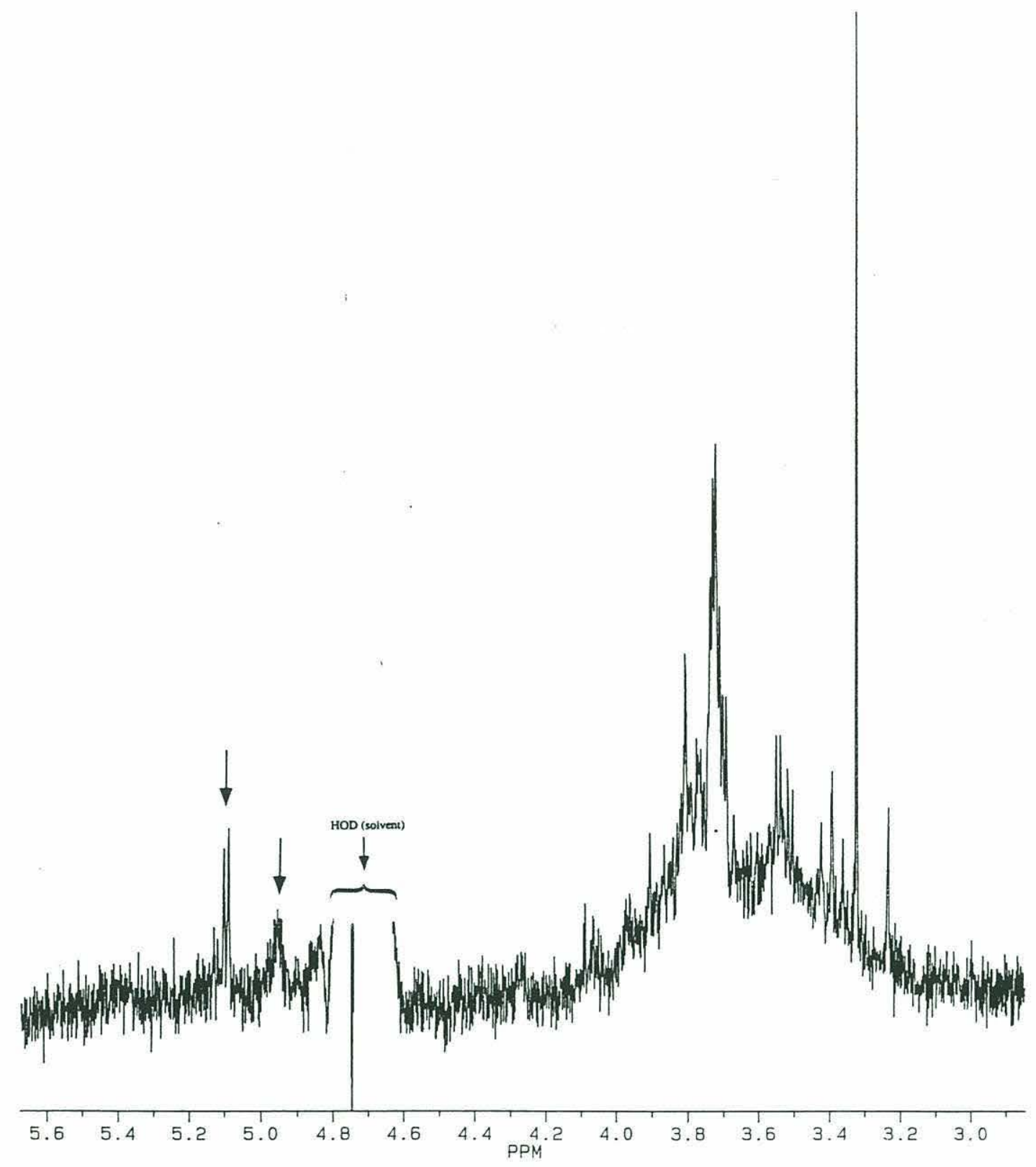

Fig. 3.7

${ }^{1}$ H NMR spectrum of F4 (48-64 minutes, GPC column) from Synechococcus WH7335 cold aqueous extract. 


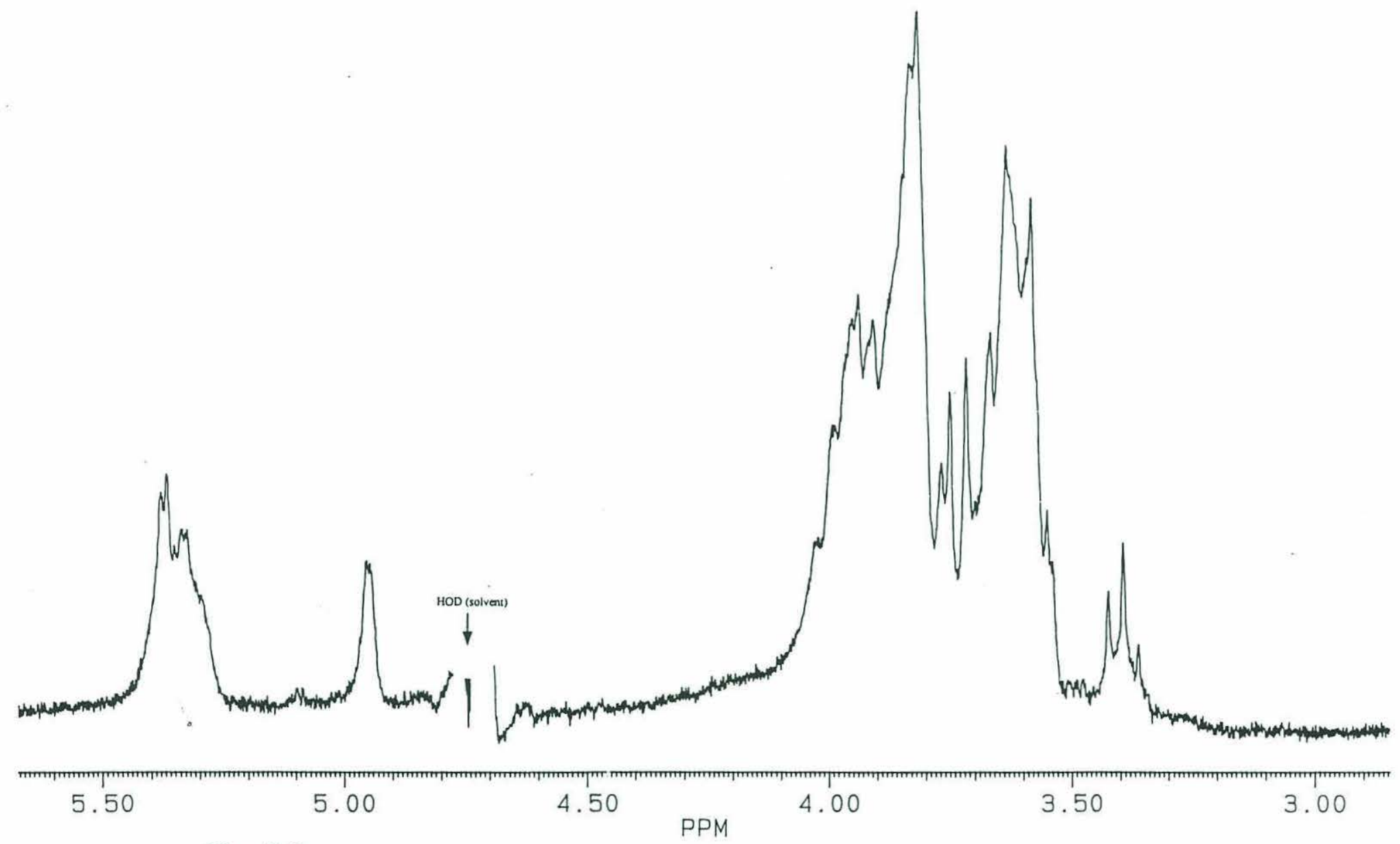

Fig. 3.8

${ }^{1} \mathrm{H}$ NMR spectrum of F2 (22-31 minutes, GPC column) from Synechococcus WH7335 cold aqueous extract. 


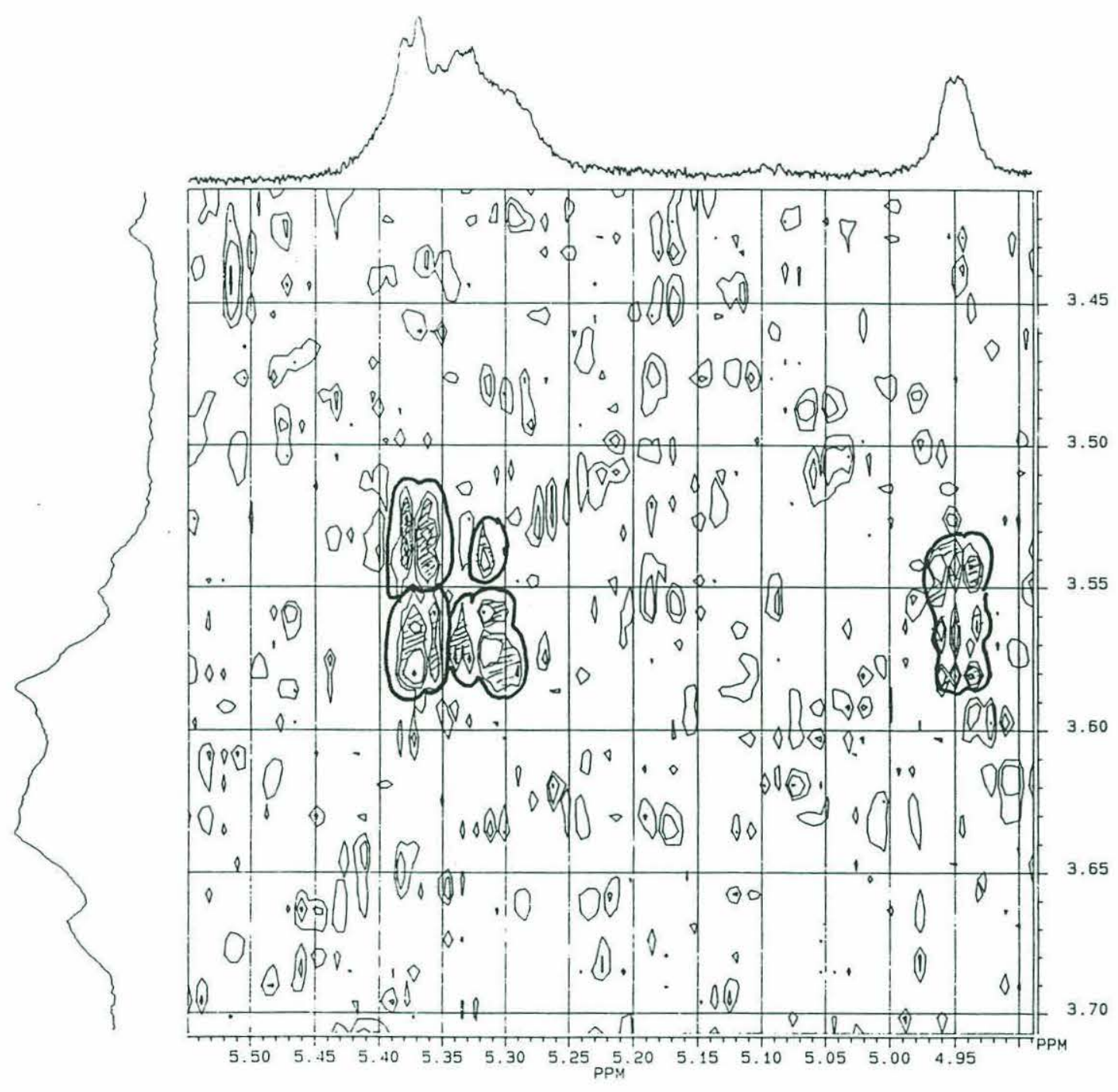

Fig. 3.9

Anomeric region of ${ }^{1} \mathrm{H}$ COSY of F2 from Synechococcus WH7335. 
Since branches generally slow the passage of a polysaccharide through a gel column, the molecular weight estimate for the polymer $(5-6 \mathrm{kD})$ represents a minimum.

Because F2 is composed of glucose, the linkage and branching positions can be determined by comparing the chemical shifts of the anomeric protons with those of glucose polysaccharide standards. The proposed structure of $F 2$, an $\alpha(1,4)$-linked glucose polysaccharide with $\alpha(1,6)$ branches, is shown in Fig. 3.10. The anomer at $\delta=4.95$ in Fig. 3.8 corresponds to an $\alpha(1,6)$ linkage (compare with Fig. 2.18, but note that Fig. 2.18 was referenced to the HOD peak at $\delta=4.8$, whereas Fig. 3.8 was referenced to internal acetone at $\delta=2.2$.). Likewise, the overlapping anomeric peaks clustered between $\delta=5.25-5.45$ correspond to $\alpha(1,4)$ linkages. The variations in chemical shifts of the $\alpha(1,4)$ anomers suggest that the main chain of this polysaccharide is $\alpha(1,4)$ linked, where the presence of an $\alpha(1,6)$ branch slightly changes the chemical shift of the $\alpha(1,4)$ unit to which it is attached. In addition, the ratio of integrated areas of the $\alpha(1,4)$ anomer and the $\alpha(1,6)$ anomer is 4.2: 1.0 , consistent with an $\alpha(1,4)$-linked main chain with $\alpha(1,6)$ branches. The frequency of branching will depend upon the size of the branches; two-unit branches (an $\alpha(1,6)$ branch with an attached $\alpha(1,4)$ glucose), for example, would on average have branches on every third glucose in the main chain, as shown in Fig. 3.10.

Finding an $\alpha(1,4)$ glucose polysaccharide with $\alpha(1,6)$ branches in the extract of Synechococcus WH7335 is consistent with other reports on the carbohydrates of cyanobacteria. As discussed above, cyanobacteria accumulate branched glucans as energy reserves, and Synechococcus sp. PCC6301 was also found to have a branched glucose polysaccharide (Bertocchi et al., 1990). These polysaccharides can comprise up to 10-30\% of total cell dry weight (Shivley, 1988), but in Synechococcus WH7335 they were only a minor fraction of the cold aqueous extract. Either Synechococcus WH7335 was harvested before it accumulated significant carbohydrate reserves, or the majority of the branched glucose polysaccharide was not removed in the cold aqueous extract. Since aqueous extractions at moderate temperatures have previously been used to obtain cyanobacterial storage products (Weber and Wober, 1975; Shivley, 1988), Synechococcus WH7335 probably had relatively low carbohydrate reserves at the time of extraction. The second culture of Synechococcus had lower concentrations of F2 than the first culture, which also implies that level of energy reserves can vary between cultures. 


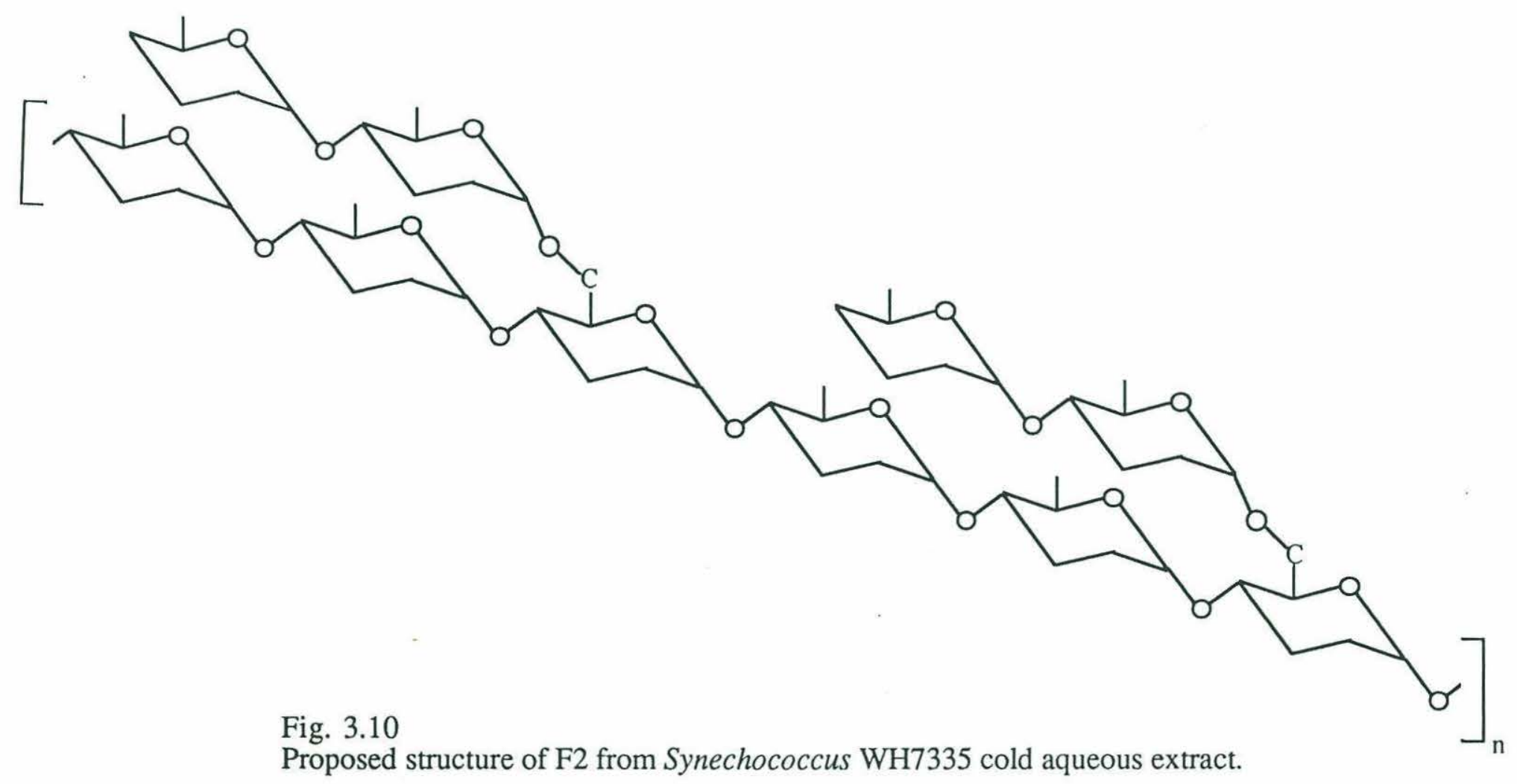

Fig. 3.10

Proposed structure of F2 from Synechococcus WH7335 cold aqueous extract. 


\section{Fraction 3}

\section{Correlation Spectroscopy (COSY)}

Fraction 3, which had an elution profile similar to that of oligosaccharides such as maltoheptaose (MW 1153), made up the majority of the cold aqueous extract (Fig. 3.6). As Fig. 3.11 shows, the proton spectrum of this fraction is very similar to Fig. 3.5, the spectrum of the total extract. Six well resolved $\alpha$-anomeric resonances and one small possible $\beta$-anomeric resonance are clearly distinguishable. The resonances in general are quite sharp, consistent with a DP3-7 oliogosaccharide (see for example maltotriose, Fig. 2.13). The only unusual feature is the lack of a prominent free $\beta$-anomeric resonance, since even for a DP7 oligosaccharide, a significant proportion of the free reducing end of the oligosaccharide (which is $1 / 7$ th of the total anomeric resonances) should be in the $\beta$-form (see Table 2.1).

A well-resolved proton COSY of F3 (Fig. 3.12) provided the starting basis for determining the major components of F3. (Note that this spectrum was acquired as a COSY 90 , with a P2 pulse of $90^{\circ}$, for enhanced sensitivity.) The major $\alpha$-anomeric resonances all gave strong cross peaks, and the anomer at $\delta=5.1$ was resolved into two overlapping doublets coupled to \#2 protons at $\delta=3.71$ and $\delta=3.53$ (Fig. 3.13). Occasionally these sorts of crosspeaks are observed as a type of 'spillover' from the anomeric proton, i.e., longrange coupling with the \#3 proton, as well as the \#2 proton, results in a crosspeak at the corresponding chemical shift along the F1 axis (see the $\alpha$-anomeric resonances of isomaltose COSY, Fig. 2.8, for example). In this case, however, close examination of the major resonance at $\delta=5.1$ revealed a slight asymmetry on the upfield side, suggesting the presence of two overlapping resonances.In addition, the smaller crosspeak at $\delta=5.1$ seems to have a further strong crosspeak (a quartet) at $\delta=4.05$, which is consistent with it being a distinct anomeric resonance, not a result of relayed magnetization transfer.

\section{Carbon-13}

Sufficient material was available for the aquisition of a ${ }^{13} \mathrm{C}$ spectrum (Fig. 3.14). A sweep width of $200 \mathrm{ppm}$ (extending from $\delta=0-200$ ) was used initially, but since all of the major carbon resonances fell in the relatively narrow window between $\delta=58-96$, a narrower sweep width was used for acquisition of a high-resolution spectrum. 


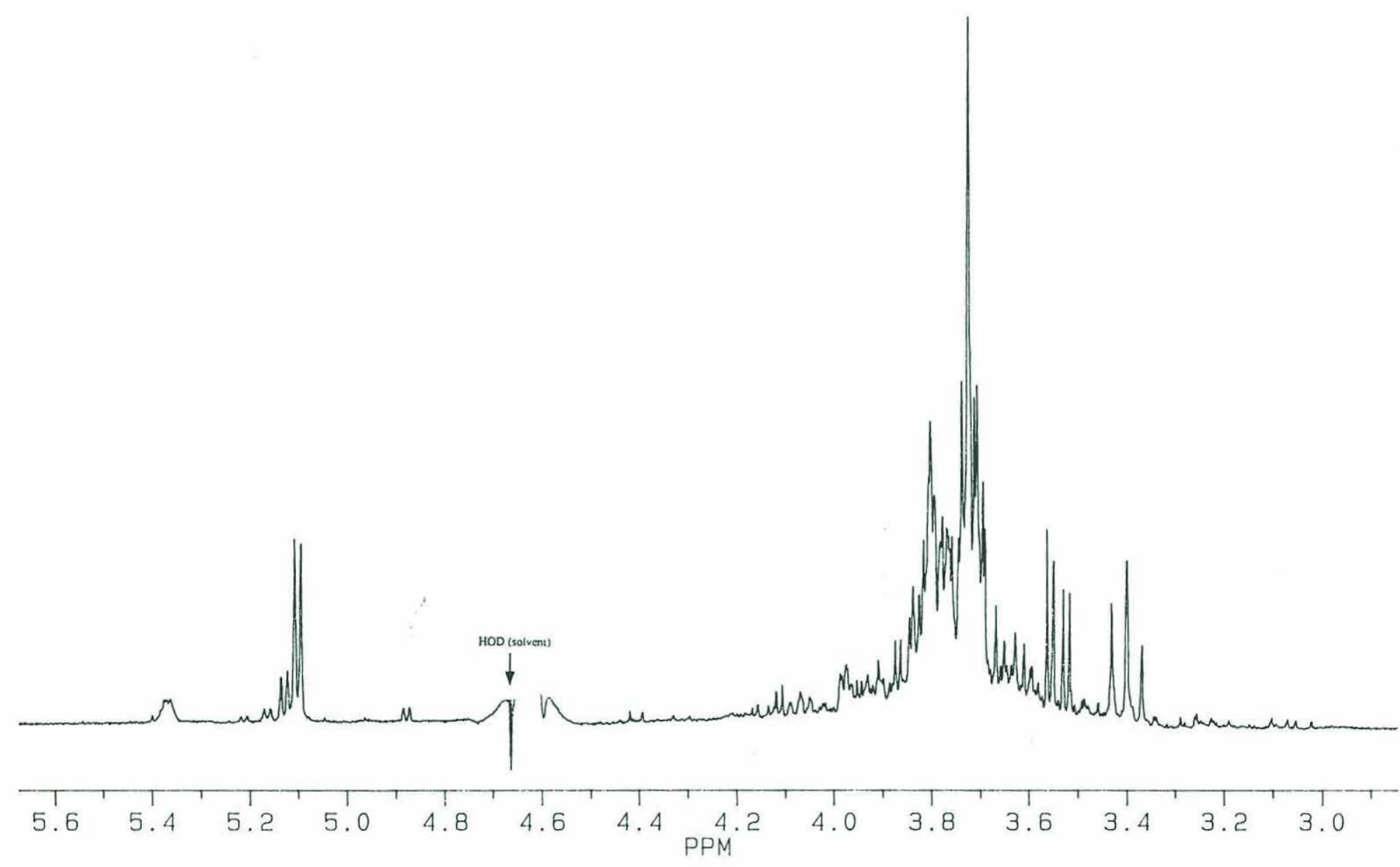

Fig. 3.11

${ }^{1} \mathrm{H}$ NMR spectrum of F3 (31-48 minutes, Sephadex G-50 GPC column) from

Synechococcus WH7335 cold aqueous extract. Referenced to acetone $(\delta=2.2)$. 


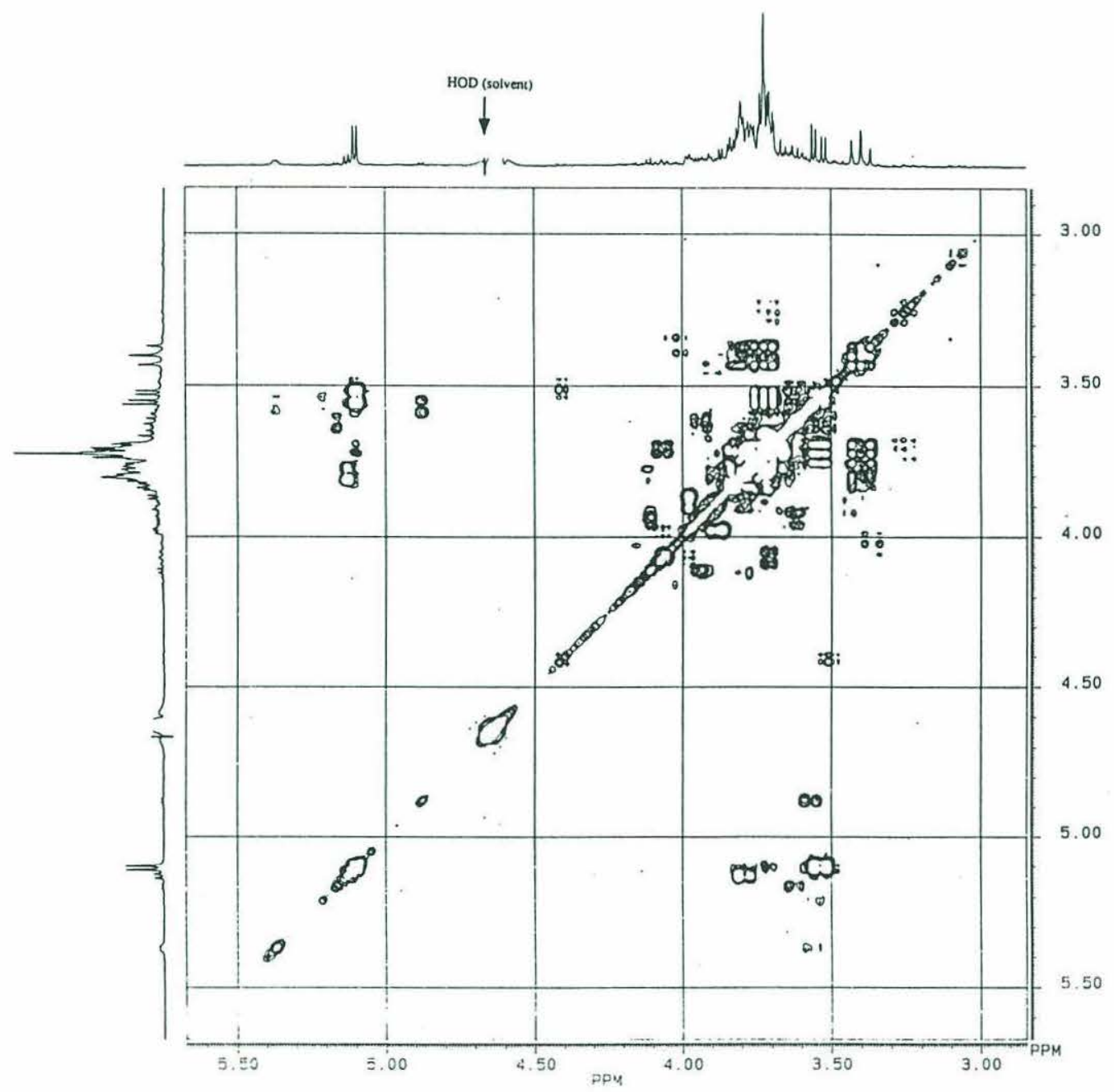

Fig. 3.12

${ }^{1} \mathrm{H}$ COSY 90 of F3 from Synechococcus WH7335 cold aqueous extract. Referenced to acetone $(\delta=2.2)$. 


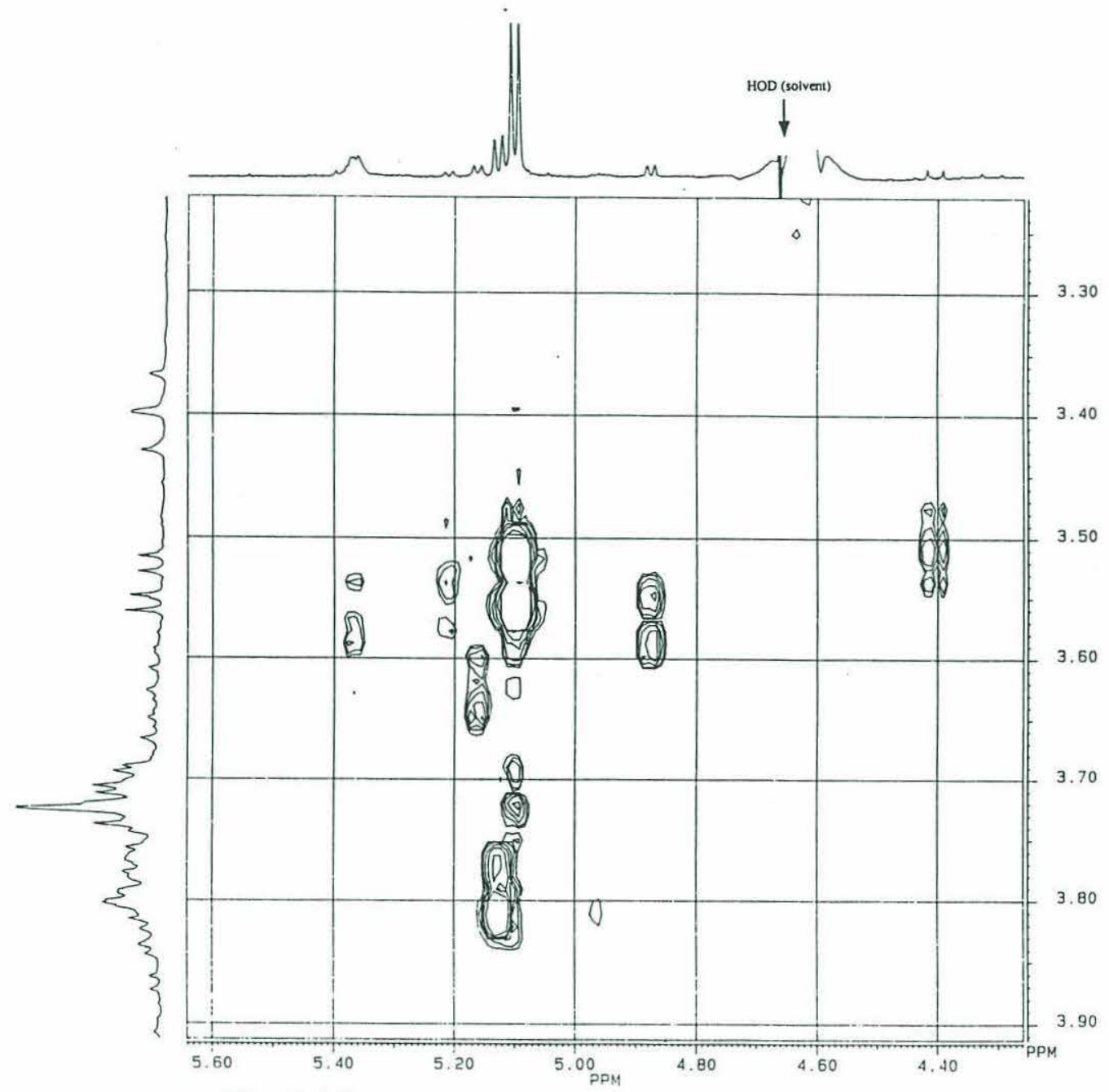

Fig. 3.13

Expansion of the anomeric region of the ${ }^{1} \mathrm{H}$ COSY of F3. 


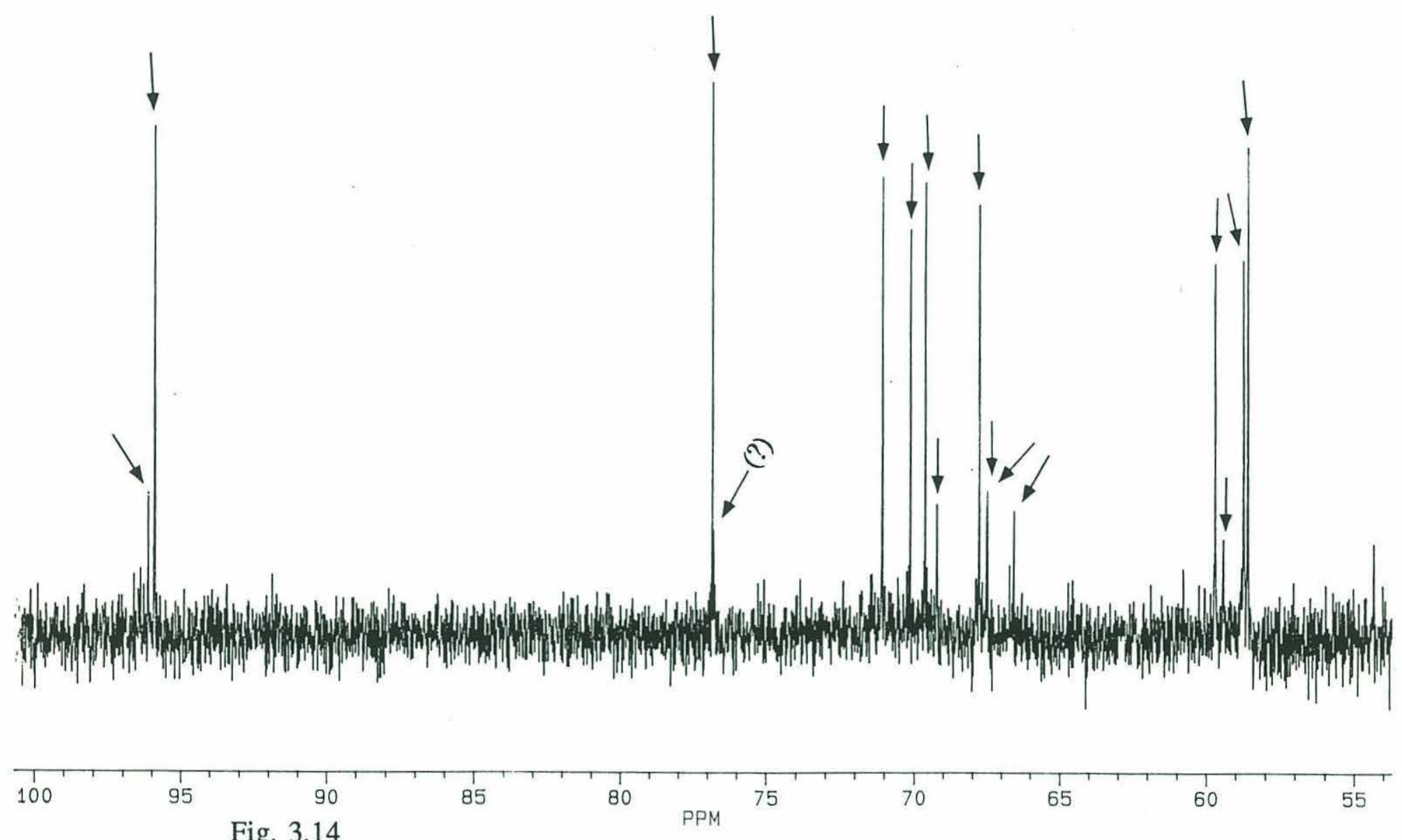

Fig. 3.14

${ }^{13} \mathrm{C}$ spectrum of F3 from Synechococcus WH7335 cold aqueous extract. Arrows mark carbon resonances. Temperature $=298 \mathrm{~K}$. Referenced to acetonitrile $(\delta=117.2)$. 
Nine major and seven minor* carbon resonances were detected (arrows, Fig. 3.14). Because no major carbon resonances were detected in the region between $\delta=15-20$, the major carbohydrate components cannot include fucose or rhamnose, since the \#6 carbon of these carbohydrates is a methyl group with a chemical shift in this region. Similarly, the lack of a carbon resonance in the region $\delta=170-180$ indicates that uronic acids are not a major component of F3 (Bock and Pederson, 1983; Bock et al., 1984). The shift range corresponds well with that expected of neutral carbohydrates, in which anomeric carbons are generally found in the range $\delta=90-104$, and \#6 carbons are usually found in the range $\delta=60-69$, with the \#2, \#3, \#4, and \#5 carbon resonances falling in between. Two carbons (one major and one minor) have chemical shifts characteristic of anomeric carbons, while four carbons (three major and one minor) have shifts close to the region where \#6 carbons typically resonate. The carbons at $\delta=58-59$ are slightly upfield from the usual range of \#6 carbons, and could, in fact, correspond to \#7 carbons-methyl groups attached to the ring carbons_-which are often found in the range $\delta=55-58$ (Bock et al., 1984).

\section{Distortionless Enhancement by Polarization Transfer (DEPT)}

This possibility was investigated by acquiring DEPT spectra of F3 (Fig. 3.15). Figure 3.15 b is a DEPT 45 experiment, which shows all protonated carbons. The spectrum is identical to Fig. 3.14, so all of the major carbons in F3 have attached protons. Figure 3.15a, a DEPT 135 experiment, inverts - $\mathrm{CH}_{2}$ carbons and shows - $\mathrm{CH}$ and

$-\mathrm{CH}_{3}$ carbons in their normal orientation. All four carbon resonances in the region $\delta=58-59$ are inverted, so all must be $-\mathrm{CH}_{2}$ carbons, and not $-\mathrm{CH}_{3}$ carbons. A DEPT 90 (not shown) showed that all of the carbons in the remainder of the spectrum $(\delta=66-96)$ are $-\mathrm{CH}$ carbons, not $-\mathrm{CH}_{3}$ carbons.

The DEPT spectrum clarified the types of carbons present in F3, but the manner in which the carbons are linked together is still unclear. The DEPT spectra show three major and one minor \#6-type carbon in F3, yet only two carbons (one major and one minor) have chemical shifts characteristic of anomeric carbons. It is difficult to envision an oligosaccharide structure in which the anomeric carbons have the same magnetic (NMR) environment—and therefore the same chemical shift—while their \#6 carbons have significantly different magnetic environments. In addition, if the major anomeric carbon at $\delta=95.9$ represents all of the major anomers, its peak height should be approximately equal

\footnotetext{
* The resonance marked with a question mark in Fig. 3.14 was resolved only when the spectrum was processed with a line broadening value of 0 , so its identification as an independent resonance is not positive.
} 
(a)

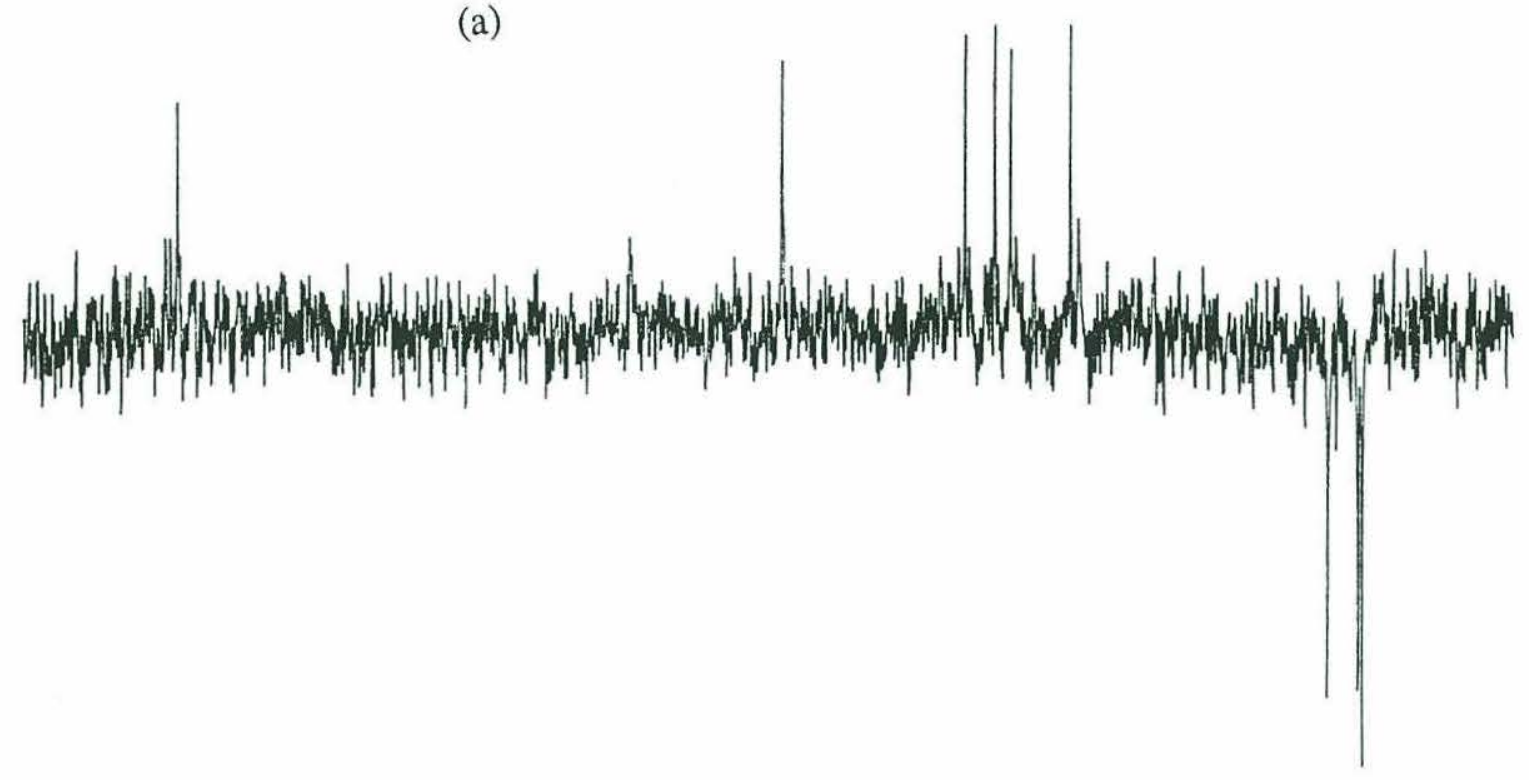

(b)

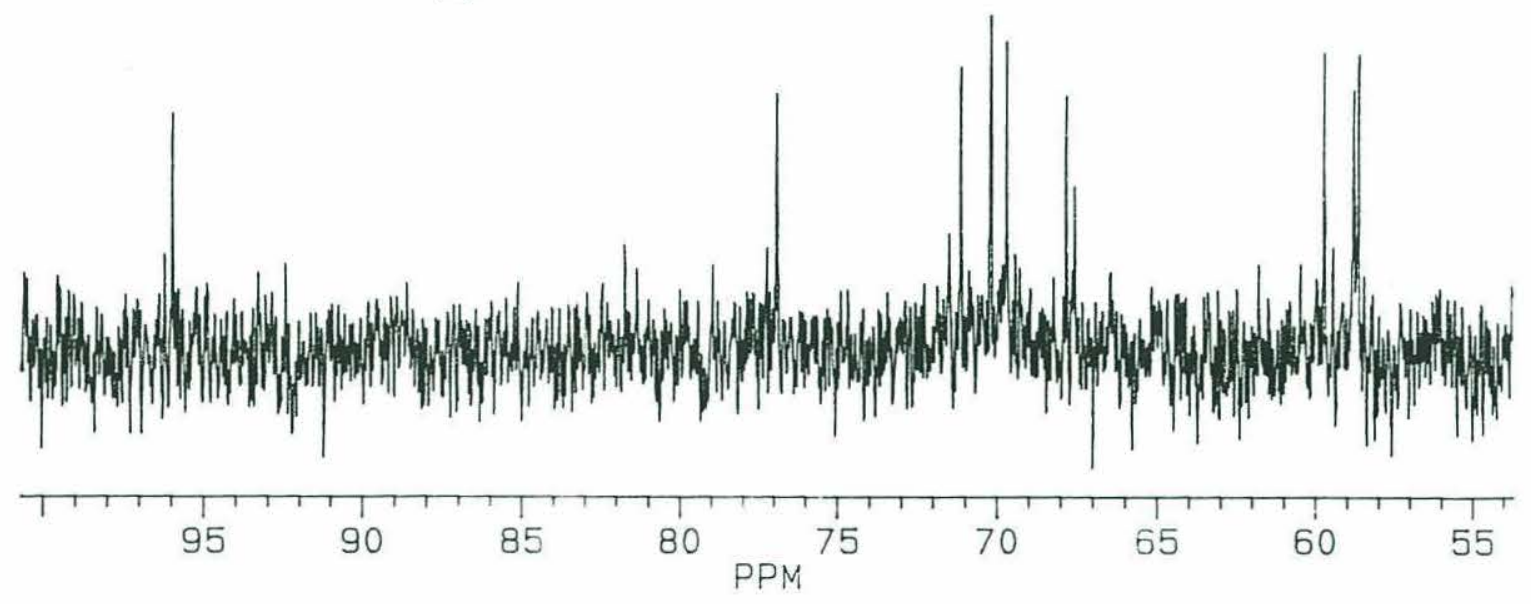

Fig. 3.15

DEPT spectra of F3. DEPT $135^{\circ}$ (a) inverts $-\mathrm{CH}_{2}$ carbons, while DEPT 450 (b) shows all protonated carbons. Referenced to acetonitrile $(\delta=117.2)$ 
to the sum of the three major \#6 carbons, which is clearly not the case. The minor anomeric carbon at $\delta=96.1$, in contrast, appears comparable in height to the minor \# 6 carbon.

\section{Heteronuclear Correlation Spectroscopy (HETCOR)}

Acqusition of a HETCOR spectrum, which correlates carbons with their attached protons, was the next step in determining the structure of the major component of F3. Figure 3.16 shows eleven distinct crosspeaks, including all nine major carbons and two of the minor carbons. From the proton spectrum along the F1 axis, the identification of the carbons at $\delta=95.88$ (the major $\alpha$-anomer, with a proton shift $\delta=5.1$ ) and 96.10 (a secondary $\alpha$ anomer, with proton shift $\delta=5.12$ ) as anomeric carbons is confirmed. They are also therefore both $\alpha$-anomeric carbons; F3 does not have a significant $\beta$-anomeric carbon component.

The DEPT 135 spectrum showed that all four crosspeaks in the region $\delta=58-59$ correspond to \#6 carbons; their proton chemical shifts overlap in the region $\delta=3.6-3.85$. The remaining five crosspeaks must correspond to \#2, \#3, \#4, and \#5 carbons. The proton COSY can aid in determining their identity. Since the carbon spectrum will include only the most abundant components of F3, only the strongest resonances in the proton COSY need to be considered. Beginning with the strongest anomeric resonance (chemical shift of $\delta=5.1$, along line A in Fig. 3.17), a strong crosspeak is found at the intersection with line B. The \#2 proton must therefore be the doublet of doublets at $\delta=3.54$, at the end of line B. Continuing to the right along line $\mathrm{B}$, a very strong crosspeak from the connection of the \#2 and \#3 protons is found at the intersection of lines B and C. The \#3 proton therefore has a chemical shift of $\delta=3.73$. Backtracking along line $\mathrm{C}$ to the intersection with line $\mathrm{D}$ leads to another strong crosspeak, which must be the crosspeak from the \#3 and \#4 protons. The well-resolved triplet at $\delta=3.4$ therefore comes from the \#4 proton. The crosspeak of the \#4 and \#5 protons is more difficult to identify, but an enlargement of the region (Fig. 3.18) shows several low-resolution crosspeaks (circled) at the intersection of lines $\mathrm{D}$ and $\mathrm{E}$. The \#5 proton must therefore be in the region around $\delta=3.8$.

Returning to an expansion of the F3 HETCOR (Fig. 3.19), the proton assignments along the F1 axis unambiguously identify the major carbohydrate carbons, with one exception. The chemical shift of the \#5 proton, which was determined to be centered around $\delta=3.8$, could correspond to the crosspeak at $\delta=70$ or at $\delta=76.8$. A search of the carbohydrate NMR literature ((Gorin, 1981)(Bock and Pederson, 1983)(Bock et al., 1984)(McIntyre et al., 1990)(McIntyre and Vogel, 1990), for example) indicated that the \#5 carbon almost certainly corresponds to the resonance at $\delta=70$. 


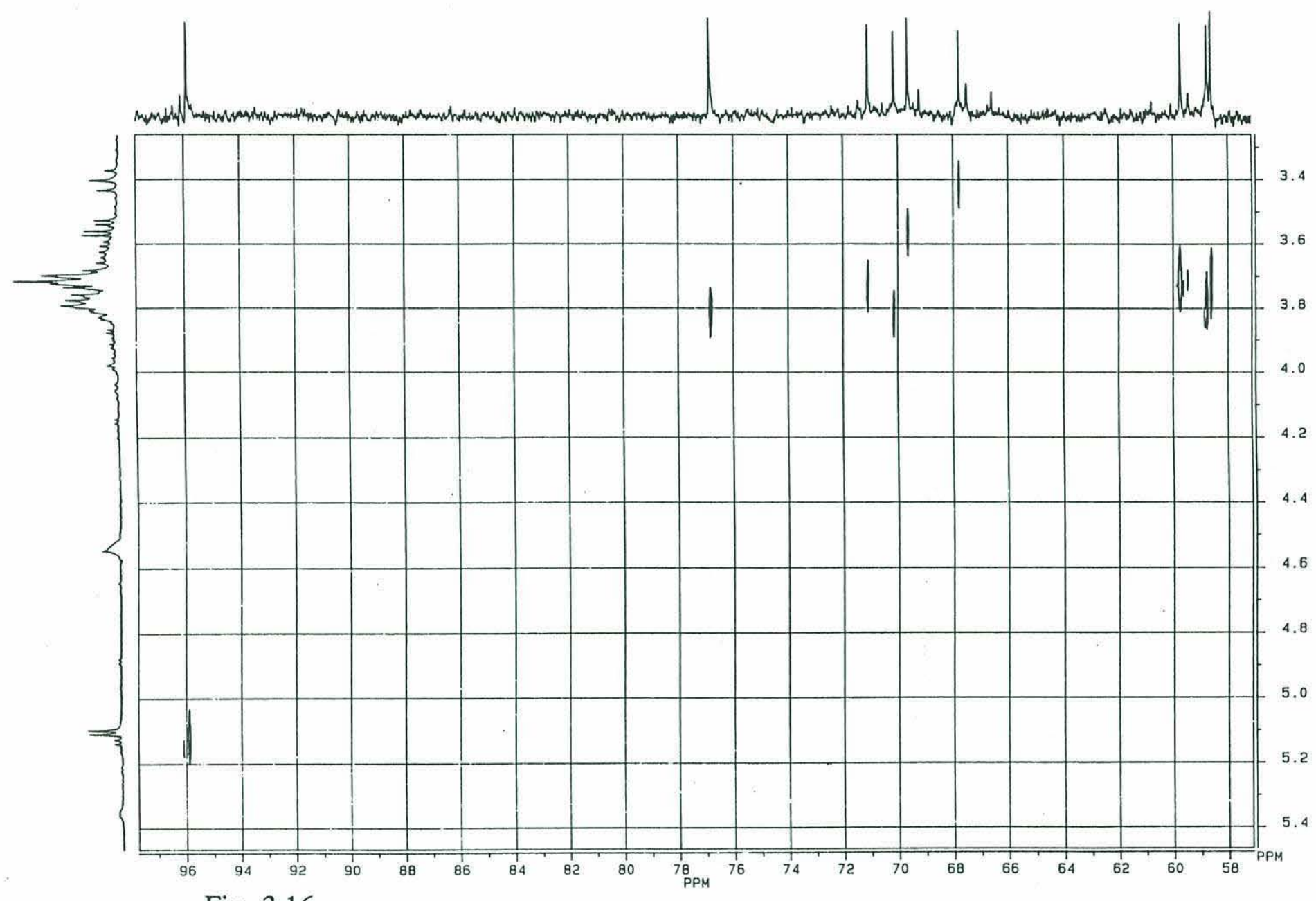

Fig. 3.16

${ }^{1} \mathrm{H}-{ }^{13} \mathrm{C}$ HETCOR of F3 from Synechococcus WH7335 cold aqueous extract. Crosspeaks show correlations of proton and carbon resonances. ${ }^{13} \mathrm{C}$ referenced to acetonitrile $(\delta=117.2)$ and ${ }^{1} \mathrm{H}$ referenced to acetone $(\delta=2.2)$ 


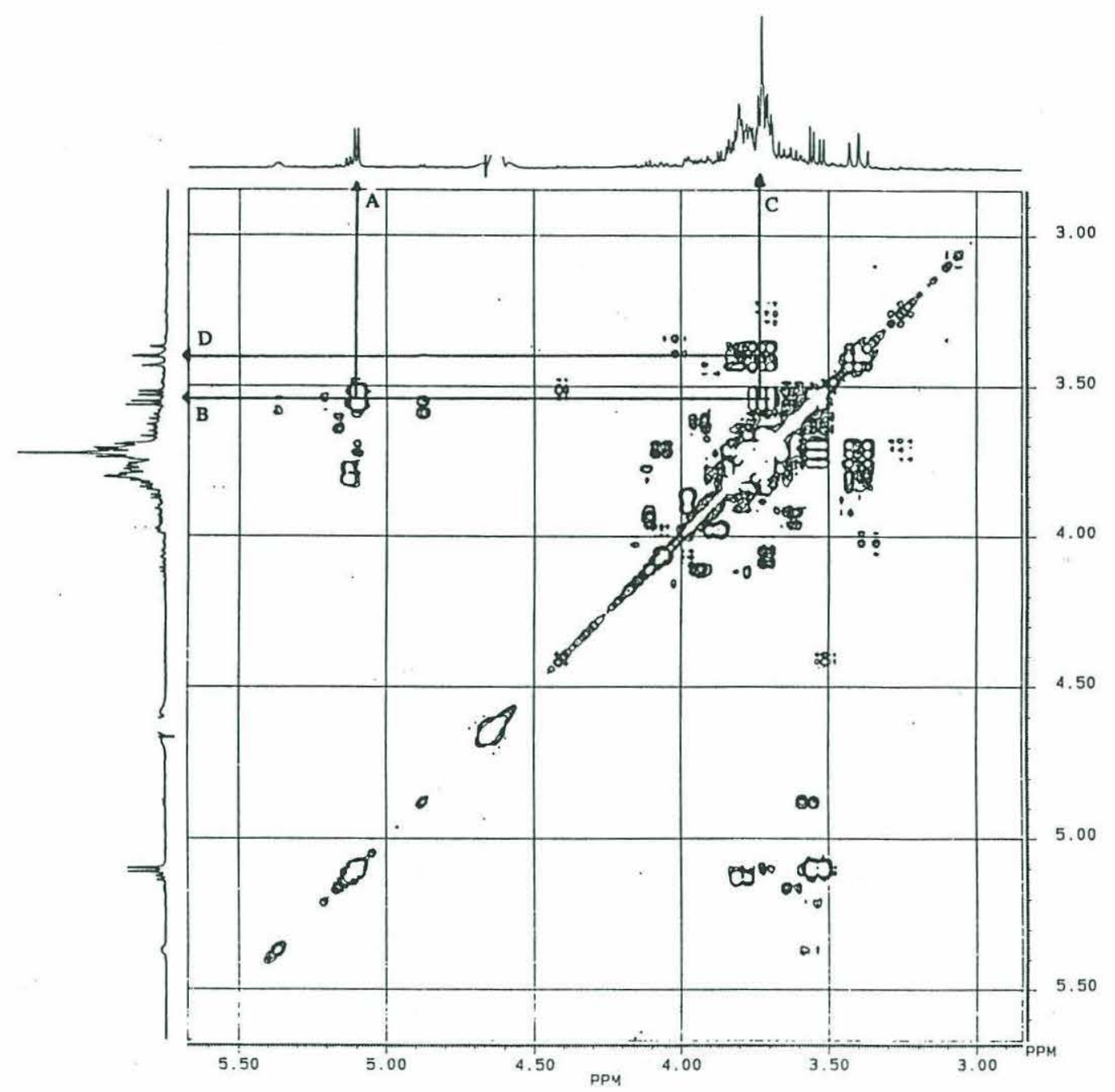

Fig. 3.17

${ }^{1} \mathrm{H}$ COSY of F3 from Synechococcus WH7335 cold aqueous extract. Lines show correlation of major proton resonances. 


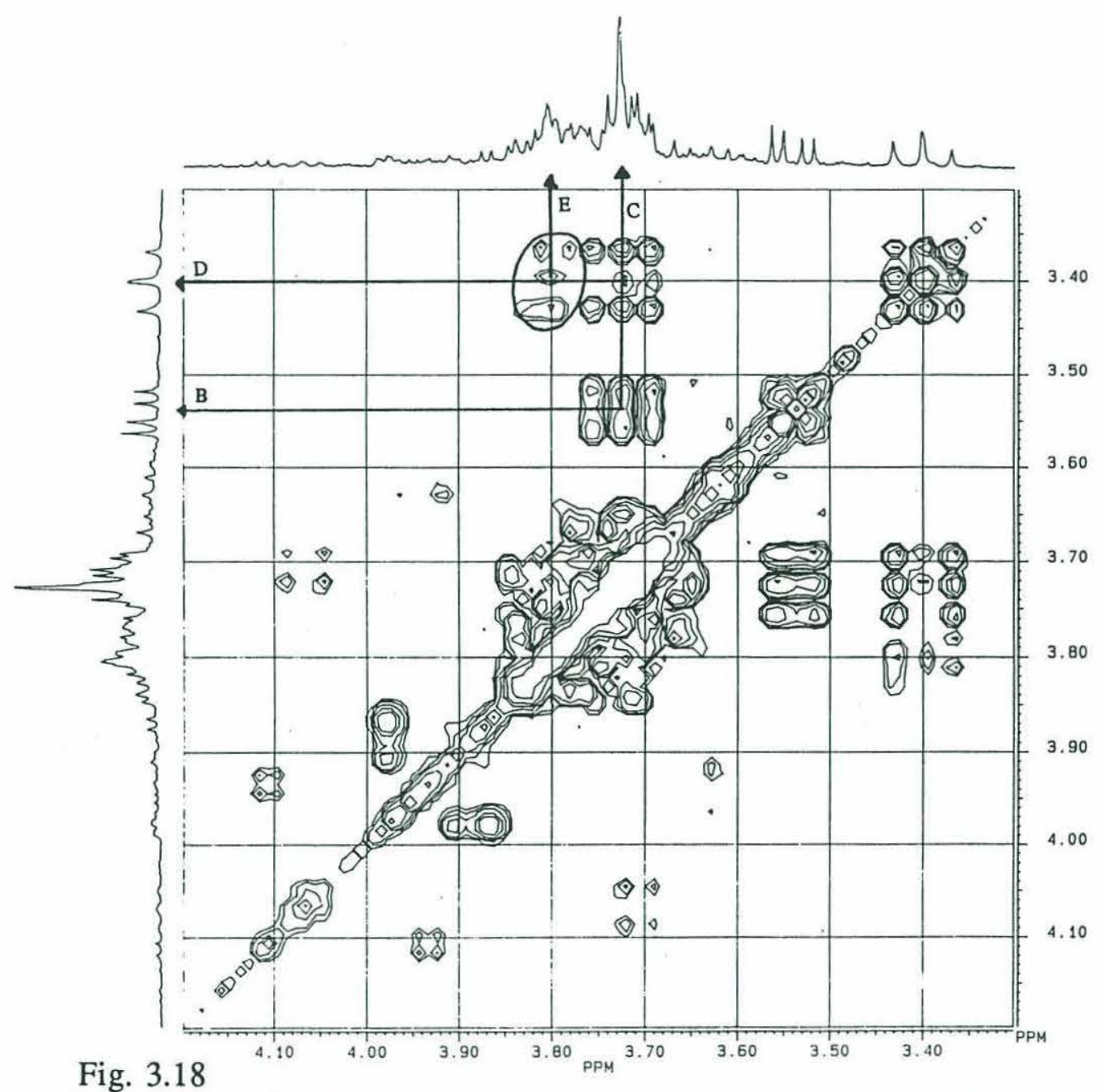

Expansion of ${ }^{1} \mathrm{H}$ COSY of $\mathrm{F} 3$, showing crosspeaks of $\# 4$ and $\# 5^{1} \mathrm{H}$ resonances. 


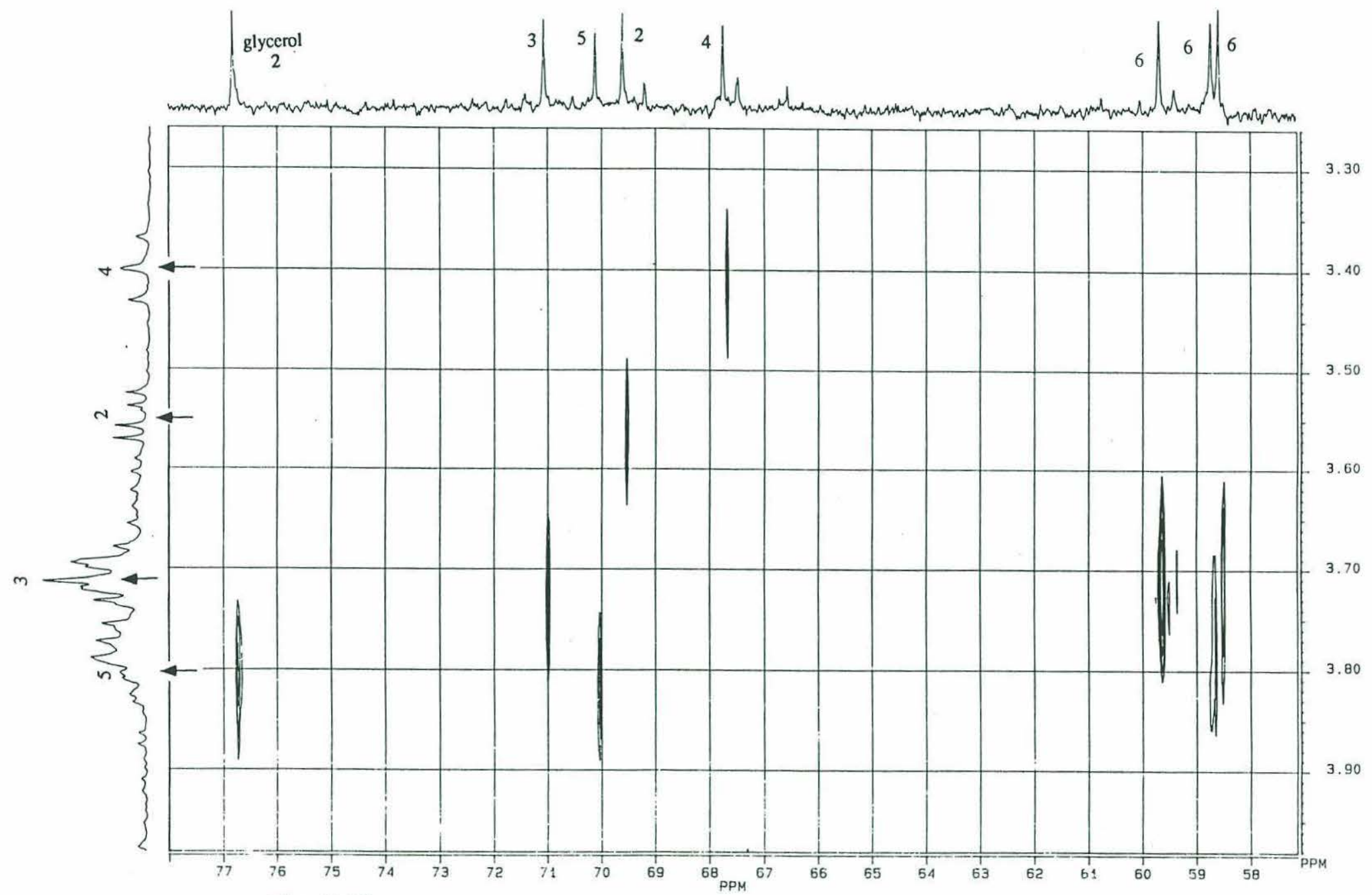

Fig. 3.19

Expansion of F3 HETCOR, showing assignment of major carbons and protons. 
At this point, the identity of the remaining carbon with chemical shift $\delta=76.8$ is uncertain. The principle problem, however, is to determine the structure of a carbohydrate with a nominal molecular weight in the range of 1000-3000 daltons which has no free $\beta$ anomers detectable either in proton or carbon spectra. In addition, this structure apparently has three major distinct types of \#6 $\left(-\mathrm{CH}_{2}\right)$ carbons, even though it has only one major anomeric carbon.

\section{Compositional and Linkage Analysis}

Compositional and linkage analysis of $\mathrm{F} 3$ at the Complex Carbohydrate Research Center (University of Georgia, Athens GA) provided some of the pieces to solve the structural puzzle. To determine monomer composition, alditol acetates of the hydrolyzed sample were analyzed by gas chromatography-mass spectrometry (GC-MS). Linkage positions were determined by Hakomori methylation and GC-MS of the permethylated alditol acetates. Table 3.2 shows that $\mathrm{F} 3$ was composed of glucose, galactose, mannose, and glycerol in approximate ratios of 1.0:0.3:0.07:0.6. The reported concentration of glycerol may be an underestimate, since triacetate derivatives of glycerol are volatile (Dr. R. Carlson, pers. comm.). These components comprised $51 \%{ }^{*}$ of F3; the remainder is presumably salt and the non-carbohydrate material seen at high field in the ${ }^{1} \mathrm{H}$ NMR spectrum. Further chemical analyses carried out at the Complex Carbohydrate Research Center demonstrated that pentoses, uronic acids, amino sugars, and acid-labile carbohydrates such as 3,6-anhyrohexose were not components of F3. These data are consistent with the results of the ${ }^{13} \mathrm{C}$ spectrum (Fig. 3.14), which indicated that the principle components of F3 were neutral hexoses. Linkage analysis showed that all three positions of the glycerol were substituted; there were no free hydroxyl groups. Mannose and galactose were present only in "terminal" forms, i.e. were linked to other components only through the \#1position. Glucose was present in two forms, 4-linked glucose (i.e., $(1,4)$ linkages) and terminal glucose, in an approximate ratio of 2:3. Glucose, galactose, and mannose are all common components of cyanobacterial cell walls, but glycerol has not previously been reported to be a component of cyanobacteia carbohydrates or glycolipids (Schmidt et al., 1980; Drews and Weckesser, 1982; Vaidya and Mehta, 1989; Bertocchi et al., 1990).

\footnotetext{
* The relative carbon content of F3 analyzed at the Complex Carbohydrate Research Center is much higher than for the sample of F3 used in CHN analysis. This is probably due to inhomogenaity of lyophilized F3. Most of F3 was used in the culture series described in Chapter 5 and for monomer and linkage analysis; only a small residue, which may have been relatively high in salt content, was available for CHN analysis.
} 


\begin{tabular}{|c|c|}
\hline glycosyl residue & mole\% \\
\hline 1,2,3-linked glycerol & 29 \\
\hline terminal mannose & 4 \\
\hline terminal galactose & 16 \\
\hline $\begin{array}{l}\text { glucose } \\
\text { (terminal glucose } \\
\text { (4-linked glucose }\end{array}$ & $\begin{array}{c}51 \\
\sim 32) \\
\sim 19)\end{array}$ \\
\hline
\end{tabular}

Table 3.2

Results of composition and linkage analysis of cold aqueous extract F3 of Synechococcus WH7335. (See text for analytical procedures.) Percentages of terminal- and 4-glucose are calculated based on relative peak areas of the GC-MS chromatogram acquired for linkage analysis. 


\section{The Structure of F3}

The proposed structure of F3 is a glycerol-phosphate oligosaccharide (Fig. 3.20). The main chain of the oligomer consists of glycerol linked through a pair of phosphoric diesters which sandwich an $\alpha(1,4)$-linked glucose unit. Monomers of glucose, galactose, and mannose are linked to the $\# 2$ carbon on each glycerol unit, and to the terminal ${ }^{* *}$ glycerol positions on either end of the chain.

The proposed structure is a variation on teichoic acids found in the cell walls of gram-positive bacteria. Teichoic acids are polymers of either ribitol phosphate or glycerol phosphate (although a mannitol phosphate teichoic acid has also been identified (Kenne and Lindberg, 1983)), in which repeating units are linked together through the phosphoric diesters. Glycerol-containing teichoic acids are the most common types of teichoic acids (Kennedy and White, 1988). Some teichoic acids have neutral carbohydrates, amino acids, or amino sugars linked to the ribitol or glycerol unit. Teichoic acid can comprise $20-40 \%$ of cell wall dry weight of gram-positive bacteria (Lehninger, 1975). As discussed previously, cyanobacteria share characteristics of both gram-positive and gram-negative bacteria, and the thick cyanobacterial cell wall is similar in construction to that of gram-positive bacteria (Weckesser and Jurgens, 1988).

Most of F3 was used as a substrate in the polysaccharide degradation experiments (Chapter 5); the small amount of remaining material was tested for the presence of phosphorus using a standard colorimetric test (Koroleff, 1983). A sample containing 1965 $\mu \mathrm{g}$ of F3 was hydrolyzed in $42 \mu \mathrm{L} 1.2 \mathrm{M} \mathrm{H}_{2} \mathrm{SO}_{4}$ for 3 hours at $100{ }^{\circ} \mathrm{C}$. The solution was then diluted with $958 \mu \mathrm{L} \mathrm{Q}-\mathrm{H}_{2} \mathrm{O}$ to reduce the $\mathrm{H}_{2} \mathrm{SO}_{4}$ concentration to $0.05 \mathrm{M}$, and phosphate concentration was measured. Calibration with standards showed that the solution contained $6.67 \mu \mathrm{M}$ phosphate, which is equivalent to $413 \mu \mathrm{g}$ phosphorus in 1965 $\mu \mathrm{g}$ of F3. If the proposed structure for F3 (Fig. 3.20) is correct, then F3 should contain approximately $9 \% \mathrm{P}$ or $\sim 180 \mu \mathrm{g} \mathrm{P}$, assuming $100 \%$ purity. Sufficient phosphorus is available to account for the phosphoric diesters in the proposed structure. Since the exact carbon content of the $\mathrm{F} 3$ residual is uncertain (see Chapter 5), stoichiometric quantification of $\mathrm{P}$ and $\mathrm{C}$ will have to be carried out on material from a fresh culture of Synechococcus WH7335. Biochemical considerations and a survey of the literature strongly suggest that

\footnotetext{
** Glycerol has three carbons; the 'terminal' positions are the \#1 and \#3 carbons, while the \#2 carbon is in the middle.
} 


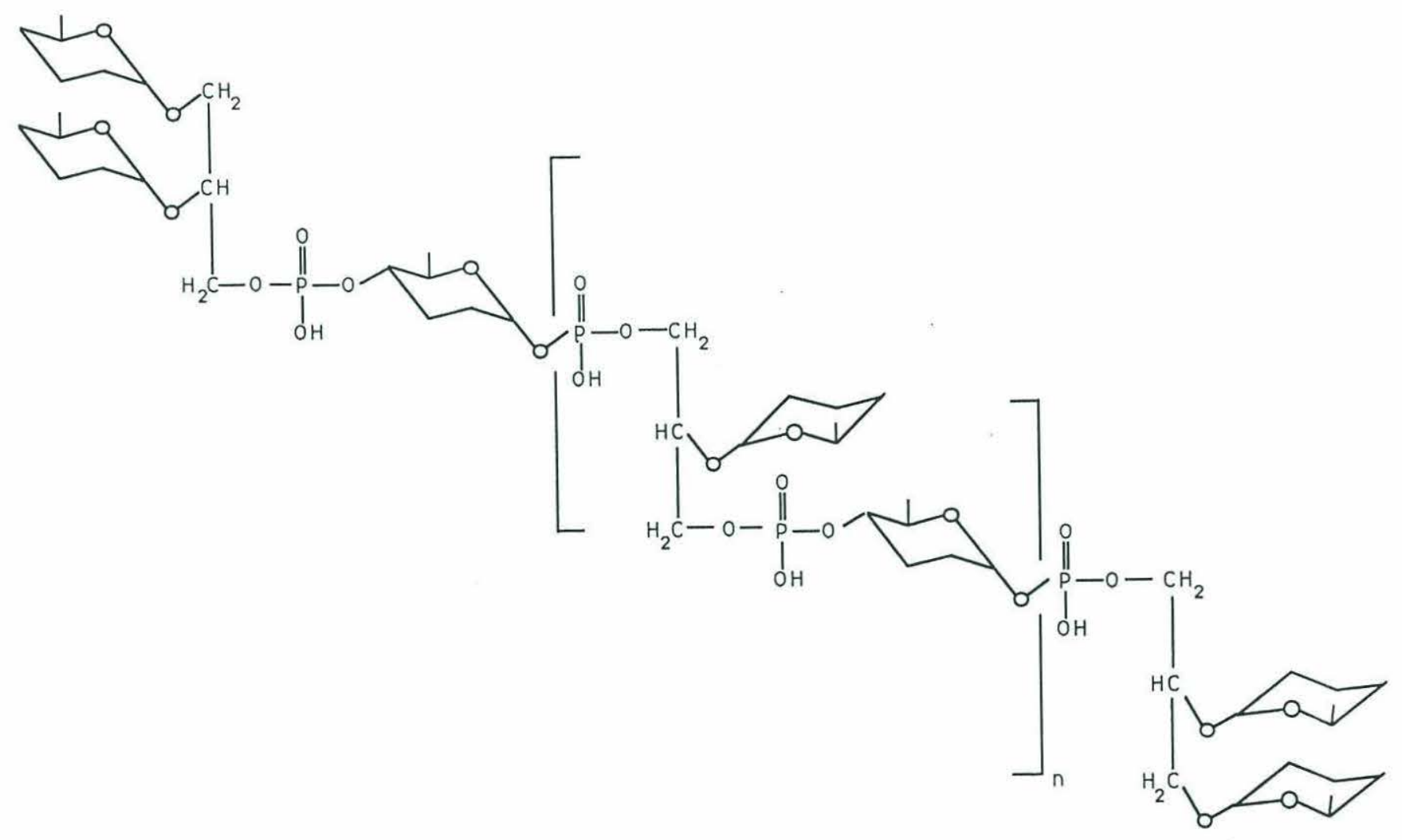

Fig. 3.20

Proposed structure of F3 from Synechococcus WH7335 cold aqueous extract: a glycerolphosphate oligosaccharide, similar in structure to teichoic acids of gram-positive bacteria 
the phosphorus in F3 is most likely contained in phosphoric diesters; ${ }^{31} \mathrm{P}$ NMR studies should provide unambiguous structural identification.

The only other glycerol-oligosaccharide structures known to occur in bacteria are glycolipids, which have long-chain fatty acids esterified to the \#1 and \#2 carbons of glycerol, and a mono- or oligosaccharide glycosydically linked to the \#3 carbon. The NMR spectra of F3, however, are not consistent with a major lipid component, because resonances characteristic of lipids were completely absent in all of the ${ }^{13} \mathrm{C}$ and ${ }^{1} \mathrm{H}$ spectra. In addition, extraction of glycolipids is usually carried out with organic solvents. Because of the size of their hydrophobic moiety, glycerol glycolipids would probably not be found in the cold aqueous extract of Synechococcus WH7335. The phosphate-containing structure in Fig. 3.20, in contrast, should be quite hydrophilic, is similar to known structures found in gram-positive bacteria, and is consistent with the NMR spectra as well as the linkage and compositional data.

A number of glycerol teichoic acids contain glycosyl residues in the main polymer chain. Teichoic acids with two phosphoric diesters sandwiching a glycosyl residue, similar to the structure in Fig. 3.20, have been found in species of Staphylococcus and Micrococcus (Kennedy and White, 1988). Most glycerol teichoic acids are connected through phosphoric diester linkages between the \#1 and \#3 carbons of successive glycerol units, but linkages between the \#1 and \#2 carbons have been found in teichoic acids of Bacillus subtilis and Streptomyces antibioticus (Kenne and Lindberg, 1983). The assignment of the \#1 and \#3 carbons of glycerol to phosphoric ester linkage positions in Fig. 3.20 is based both on spatial considerations and on NMR spectra which suggest that the \#2 carbon has a direct glycosyl linkage, as explained below. The structure proposed in Fig. 3.20 is sterically somewhat crowded, which may restrict free movement in solution. The bulky structure, along with a high concentration of glycerol, may help account for the high viscosity of the Synechococcus medium, and the mat-like cohesion of the cells.

The speculative feature of the proposed structure is the presence of terminal glycosyl residues attached to the \#2 carbon of glycerol. While teichoic acids with carbohydrate units directly linked to glycerol have been isolated, none of these structures also contained carbohydrate units between pairs of phosphoric diesters as part of the main polymer chain. The compositional data and the NMR spectra, however, support the proposed structure. An oligomer of $\sim 3400$ daltons (six glycerol units, $n=2$ in Fig. 3.20) would have approximately correct glycerol:carbohydrate ratios, would conform to the elution profile of F3 on the G50 GPC column, and is consistent with the NMR spectra. The 'oligosaccharide-like' appearance of the ${ }^{1} \mathrm{H}$ NMR spectrum (Fig 3.11) is due to the 
absence of direct carbohydrate-carbohydrate linkages in F3; all of the carbohydrates are linked either to glycerol or are sandwiched between phosphoric diesters.

The NMR spectra also provide compelling evidence for a direct carbohydrate linkage to the $\# 2$ carbon of glycerol. The presence of glycerol accounts for the three $-\mathrm{CH}_{2}$ groups detected in the DEPT experiment (Fig. 3.15); two carbons are from the \#1 and \#3 carbons of glycerol, while one is the \#6 carbon of glucose. The previously unassigned carbon at $\delta=76.8$ is most likely the \#2 glycerol carbon. The carbon shifts of free glycerol are $\delta=71.2$ (\#2 carbon) and $\delta=61.7$ (\#1 and \#3 carbons), however, so the chemical shift of the \#2 carbon has been shifted downfield. The \#2 glycerol carbon is chemically similar to $\# 2$, \#3, and \#4 carbons in a monosaccharide ring, and literature data shows that the chemical shifts of \#2-\#4 monosaccharide carbons are moved downfield from $\delta=70-74$ to $\delta=76-86$ when directly linked to a glycosyl unit (Bock et al., 1984). The chemical shift of the \#2 glycerol carbon, therefore, is consistent with a direct linkage to terminal glucose, galactose, or mannose.

The \#1 and \#3 glycerol carbons in F3, in contrast, are shifted somewhat upfield of their free glycerol resonance positions. Since for a monosaccharide, a direct linkage of a $-\mathrm{CH}_{2}$ type carbon to a glycosyl unit also shifts the resonance position 5-8 ppm downfield, the \#1 and \#3 glycerol carbons are probably not directly linked to a terminal monosaccharide. In the case of isomaltose, for example, the ring I \#6 carbon has a chemical shifts of $\delta=64$, while the ring II carbon which is not part of an interglycosyl linkage has a chemical shift of $\delta=59$ (Table 2.3). Linkage of the glycerol \#1 and \#3 carbons though a phosphoric ester may produce the slight upfield shift observed in the ${ }^{13} \mathrm{C}$ spectrum (Fig. 3.14). (Note that if the F3 oligosaccharide does have an approximate molecular weight of 3400 , the NMR signals of the \#1 and \#3 carbons at the ends of the oligosaccharide which do have glycosyl linkages would not be abundant enough to be resolved from the baseline of the ${ }^{13} \mathrm{C}$ spectrum.)

Since only nine resonances dominate the ${ }^{13} \mathrm{C}$ spectrum, and glucose comprises more than $50 \%$ of $\mathrm{F} 3$, the major carbohydrate resonances are assigned to glucose. The remaining three major resonances are from glycerol. The chemical shifts of six of the minor resonances observed in Fig. 3.14 are assigned to galactose, which comprises approximately $16 \%$ of $\mathrm{F} 3$ on a molar basis. The positions of the minor resonances in the ${ }^{13} \mathrm{C}$ spectrum (Fig. 3.14) supports their assignment to galactose rather than to 4-linked glucose. If the minor resonances were from 4-linked glucose, they would (with the exception of the \#4 carbon) closely correspond to the carbon shifts of the terminal glucose unit. Instead, the minor resonances cluster upfield, and have chemical shifts consistent with galactose. The assignments of the \#1 and \#6 galactose carbons are based on the proton and 


\section{8}

DEPT spectra, respectively, while the assignments of the \#2-\#5 carbons are based on comparisons with literature values (Bock et al., 1984). The most tentative assignment is of the \#3 and \#4 carbons, since the relative resonance position of these two carbons can be reversed. In this case, the two resonances nearly overlap, and assignment was made arbitrarily. The complete assignment of the ${ }^{13} \mathrm{C}$ shifts is given in Table 3.3. The resonances of the 4-linked and terminal glucose carbon most likely overlap, with one possible exception. The identification of a seventh minor carbon resonance at $\delta=76.79$ is tentative, since resolution of this resonance from the major resonance at $\delta=76.82$ is dependent upon the line broadening value used to process the FID of the ${ }^{13} \mathrm{C}$ spectrum (see footnote to Table 3.3, and references in the beginning of Chapter Two, for details). If the seventh minor carbon resonance is real, it may corresponds to the \#4 carbon of the 4-linked glucose.

Assignments of some of the anomeric protons can also be made. In Fig. 3.13, the major anomer at $\delta=5.1$ clearly belongs to glucose. Based on the ${ }^{13} \mathrm{C}$ assignment, the anomer at $\delta=5.13$ must belong to galactose. The anomeric proton of 4-glucose, which is linked to a phosphoric ester, most likely has a different chemical shift than anomeric proton of terminal glucose, which is linked to the \#2 glycerol carbon. Since 4-glucose is part of the main polymer chain, its motion is more constrained and its anomeric resonance and crosspeak would be less well defined than anomeric protons corresponding to terminal monosaccharide units. The anomer at $\delta=5.37$, the usual position for $\alpha(1,4)$ glucose linkages, therefore probably corresponds to the anomeric proton of 4-glucose. Exact identitification of the other anomers is difficult; galactose corresponds to one of the small anomers at $\delta=5.18,5.21$, or 4.95 . Since terminal monosaccharides seem to produce strong crosspeaks, and the chemical shift of $\alpha$-galactose is usually found in the region near $\delta=5.1-5.2$, the anomer at $\delta=5.18$ is most likely $\alpha$-galactose. The multiple low-intensity anomers (including the small anomer overlain by the major glucose anomer at $\delta=5.1$ ) may arise from monosaccharides attached to \#1 and \#3 glycerol carbons at either end of the oligomer. Due to differences between ${ }^{1} \mathrm{H}$ and ${ }^{13} \mathrm{C}$ in isotopic abundance and NMR sensitivity, the anomeric protons from these terminal monosaccharides would be observable, even though the corresponding anomeric carbons would not be detected.

All of the carbohydrate linkages are $\alpha$-anomers, and the lack of free $\beta$-anomers in the ${ }^{1} \mathrm{H}$ and ${ }^{13} \mathrm{C}$ spectra (Figs. 3.11, 3.14) provides strong evidence that glycerol and carbohydrates found in $\mathrm{F} 3$ are not merely co-occurring separate structures which were coincidentally freed by the same extraction procedure. An oligosaccharide would normally have a significant free reducing $\beta$ end which should be detectable in ${ }^{1} \mathrm{H}$ and ${ }^{13} \mathrm{C}$ spectra, 
chemical shift: $\quad$ relative intensity (highest peak $=1$ ) assignment

$\begin{array}{lccl}58.57 & 0.86 & \text { glycerol } & \text { terminal C* } \\ 58.72 & 0.67 & \text { glycerol } & \text { terminal C* } \\ 59.39 & 0.17 & \text { galactose } \\ 59.67 & 0.66 & \# 6 & \text { glucose* } \\ 66.56 & 0.22 & \text { galactose } \\ 67.46 & 0.25 & \# 3 & \text { galactose } \\ 67.49 & 0.23 & \text { galactose } \\ 67.75 & 0.72 & \# 4 & \text { glucose } \\ 69.20 & 0.23 & \# 5 & \text { galactose } \\ 69.60 & 0.86 & \text { glucose } \\ 70.10 & 0.77 & \# 5 & \text { glucose } \\ 71.07 & 0.75 & \text { glucose } \\ (76.79 * * & 0.20 & \# 4 & \text { 4-linked glu?) } \\ 76.82 & 1.00 & \text { glycerol } & \text { \#2 C } \\ 95.88 & 0.87 & \# 1 & \text { glucose } \\ 96.10 & 0.21 & \# 1 & \text { galactose }\end{array}$

* assignment of the terminal glycerol carbons and the \#6 glucose carbon may be reversed

**identification as an independent resonance is tentative; processing the FID with line broadening $(\mathrm{LB})=0$ produces a spectrum in which this resonance is resolved from the major resonance at $\delta=76.82$. With non-zero LB values, the base of the resonance at $\delta=76.82$ is wider, and a second resonance is not identifiable.

Table 3.3

${ }^{13} \mathrm{C}$ chemical shifts of $\mathrm{F} 3$ from Synechococcus WH7335

(temperature $298 \mathrm{~K}$; referenced to internal acetonitrile, $\delta=117.2$ ) 
but the glycerol-phosphate oligosaccharide has no free reducing ends; all carbohydrate components are linked through the anomeric carbon either to glycerol or phosphate.

Although teichoic acids are common in gram-positive bacteria, neither teichoic acids nor glycerol-containing carbohydrates of any nature have been previously reported in cyanobacteria. This lack information is probably due to the extraction and analytical procedures used in previous studies of cyanobacteria, rather than to a unique feature of Synechococcus WH7335. Little structural information other than monosaccharide composition is available for most cyanobacterial carbohydrates (Mehta and Vaidya, 1978; Vaidya and Mehta, 1989; Bertocchi et al., 1990), and the usual problems of carbohydrate identification - most procedures detect only a limited range of components-apply as well. Glycerol triacetates are quite volatile, which can impede detection by most derivitazation procedures (pers. comm, Dr. R.W. Carlson, Univ. Georgia). In addition, phosphate linkages are labile, and would be easily hydrolyzed by acid or base extraction procedures, as well as by any of the common carbohydrate derivatization procedures. Studies of cyanobacterial lipopolysaccharides also do not report any structures similar to Fig. 3.20, but since phenol (Weckesser and Jurgens, 1988) or chloroform:methanol (Chaplin and Kennedy, 1986) are usually used to extract lipopolysaccharides, a hydrophilic teichoic acid would not be extracted by these methods.

Final confirmation of the teichoic acid structure of $\mathrm{F} 3$ can be made through stoichiometric determination of the C:P ratio and ${ }^{31} \mathrm{P}$ NMR studies. Further NMR experiments designed to confirm linkage position and connectivites (nOe experiments, relay COSY, selective decoupling, etc.) could also be made. Size calibrations of branched and charged oligomers via gel permeation chromatography are problematic, since hydrodynamic volume and electrostatic interaction with stationary phases can affect elution time. Mass spectrometry may be useful in providing approximate molecular weight estimates, and fragmentation patterns could provide more information about the repeating units in the oligomer.

\section{Conclusions}

Synechococcus WH7335 produces large quantities of carbohydrates. Only $800 \mathrm{~mL}$ of culture yielded over $70 \mathrm{mg}$ of primarily carbohydrate-containing material just in the cold aqueous extract. Further carbohydrates were contained in the hot aqueous extract, and the residual cellular material probably contained more carbohydrates. In addition, the cyanobacterium excretes very high quantities of carbohydrates into the culture medium. One of the structures identified in the cold aqueous extract of Synechococcus WH7335 was 


\section{1}

an $\alpha(1,4)$-linked glucose polysaccharide with $\alpha(1,6)$ branches, probably accumulated as an energy reserve, with a minimum $\mathrm{MW}>5000$ daltons. The proposed structure of the principle carbohydrate component of the cold aqueous extract is a glycerol-phosphate oligosaccharide in which a pair of phosphoric diesters sandwich an $\alpha(1,4)$-linked glucose. The oligomer is structurally related to teichoic acids found in cell walls of gram positive bacteria. If the structural assignment is correct, this is the first report of teichoic acids in cyanobacteria.

\section{Acknowledgements:}

Thanks to Freddie Valois and John Waterbury for introducing me to Synechococcus WH7335, and for growing both cultures used in this work. Ralf Goericke grew the Dunaliella tertiolecta, and Liz Minor grew the culture of $E$. huxleyi. Mark Dennet and Joel Goldman generously provided the seed culture of Stephanopixis palmeri, and Mark, Ralf, and Freddie all helped me culture Stephanopixis and Phaeocystis. Bob Nelson did the CHN analyses, and Kathleen Ruttenberg made the phosphate measurements. The monomer and linkage analyses of F2 and F3 from Synechococcus WH7335 were carried out by the Complex Carbohydrate Research Center at the University of Georgia (Athens, GA). The analyses were partially supported by DOE funding (DE-FG05-93ER20097) to the Center for Plant and Microbial Complex Carbohydrates. 


\section{References}

Allan, G. G., J. Lewin and P. G. Johnson (1972). Marine Polymers. IV Diatom polysaccharides. Bot. Mar. 15: 102-108.

Bertocchi, C., L. Navarini and A. Cesaro (1990). Polysaccharides from cyanobacteria. Carbo. Polymers 12: 127-153.

Bishop, C. T. and H. J. Jennings (1982). Immunology of polysaccharides. The Polysaccharides. New York, Academic Press. 292-325.

Bock, K. and C. Pederson (1983). Carbon-13 nuclear magnetic resonance spectroscopy of monosaccharides. Adv. Carbo. Chem. Biochem. 41: 27-66.

Bock, K., C. Pederson and H. Pederson (1984). Carbon-13 nuclear magnetic resonance data for oligosaccharides. Adv. Carbo. Chem. Biochem. 42: 193-225.

Brown, M. R. (1991). The amino-acid and sugar composition of 16 species of microalgae used in mariculture. J. Exp. Mar. Biol. Ecol. 145: 79-99.

Burney, C. M., P. G. Davis, K. M. Johnson and J. M. Sieburth (1981). Dependence of dissolved carbohydrate concentrations upon small scale nanoplankton and bacterioplankton distributions in the Western Sargasso Sea. Mar. Biol. 65: 289-296.

Chaplin, M. F. and J. F. Kennedy (1986). Carbohydrate Analysis: A Practical Approach. Oxford, IRL Press.

Cole, J. J., G. E. Likens and D. L. Strayer (1982). Photosynthetically produced dissolved organic carbon: An important carbon source for planktonic bacteria. Limnol. Oceanogr. 27(6): 1080-1090.

Drews, G. and J. Weckesser (1982). Function, structure, and composition of cell walls and external layers. The Biology of Cyanobacteria. Berkelely, University of California Press. 333-357.

Fogg, G. E. (1977). Excretion of organic matter by phytoplankton. Limno. Oceano. 22(3): 576-577.

Frew, N. M., J. C. Goldman, M. R. Dennett and A. S. Johnson (1990). Impact of phytoplankton-generated surfactants on air-sea gas exchange. J.G.R. 95(C3): 3337-3352.

Geresh, S., O. Dubinsky, S. Arad, D. Christiaen and R. Glaser (1990). Structure of the 30 -( $\alpha$-D-glucopyranosyluronic acid)-L-galactopyranose, an aldobiouronic acid isolated from the polysaccharides of various unicellular red algae. Carbohydr. Res. 208: 301-305.

Gorin, P. A. J. (1981). Carbon-13 nuclear magnetic resonance spectroscopy of polysaccharides. Adv. Carbo. Chem. Biochem. 38: 13-97.

Guillard, R. R. and P. J. Wangersky (1958). The production of extracellular carbohydrates by some marine flagellates. Limno. Oceano. 3(4): 449-454.

Guillard, R. R. L. and J. A. Hellebust (1971). Growth and the production of extracellular substances by two strains of Phaeocystis poucheti.J. Phycol. 7: 330-338. 
Hama, T. (1988). 13C-GC-MS analysis of photosynthetic products of the phytoplankton population in the regional upwelling area around the Izu Islands, Japan. Deep Sea Res. 35(1): 91-110.

Handa, N. (1969). Carbohydrate metabolism in the marine diatom Skeletonema costatum. Mar. Biol. 4: 208-214.

Haug, A. and S. Myklestad (1976). Polysaccharides of marine diatoms with special reference to Chaetoceros species. Mar. Biol. 34: 217-222.

Haug, A., S. Myklestad and E. Sakshaug (1973). Studies on the phytoplankton ecology of the Trondjeimsfjord. I. The chemical composition of phytoplankton populations. J. exp. mar. Biol. Ecol. 11: 15-26.

Hecky, R. E., K. Mopper, P. Kilham and E. T. Degens (1973). The amino acid and sugar composition of diatom cell-walls. Mar. Biol. 19: 323-331.

Henrichs, S. M. (1991). Methods of sample handling and analysis for dissolved and particulate amino acids and carbohydrates in seawater. Marine Particles: Analysis and Characterization. Washington D.C., American Geophysical Union. 139-149.

Hitchcock, G. L. (1978). Labelling patterns of carbon-14 in net plankton during a winterspring bloom. J. exp. mar. Biol. Ecol. 31: 141-153.

Hitchcock, G. L., J. C. Goldman and M. R. Dennett (1986). Photosynthate partitioning in cultured marine phytoplankton: metabolic patterns in a marine diatom under constant and variable light intensities. Mar. Ecol. Prog. Ser. 30: 74-84.

Iturriaga, R. and A. Zsolnay (1983). Heterotrophic uptake and transformation of phytoplankton extracellular products. Bot. Mar. 26: 375-381.

Jurgens, U. J. and J. Weckesser (1986). Polysaccharide covalently linked to the peptidoglycan of the cyanobacterium Synechocystis sp. strain PCC6714. J. Bact. 168: 568-573.

Kenne, L. and B. Lindberg (1983). Bacterial Polysaccharides. The Polysaccharides. Academic Press. 287-363.

Kennedy, J. F. and C. A. White (1988). The plant, algal, and microbial polysaccharides. Carbohydrate Chemistry. Oxford, Clarendon Press. 220-262.

Kieras, J. H., F. J. Kieras and D. V. Bowen (1976). 2-O-methyl-D-glucuronic acid, a new hexuronic acid of biological origin. Biochem. J. 155: 181-185.

Koroleff, F. (1983). Determination of phosphorus. Methods of Seawater Analysis.

Weinheim, VCH. 125-139.

Lee, R. E. (1980). Phycology. Cambridge, Cambridge University Press.

Lehninger, A. L. (1975). Biochemistry. New York, Worth Publishers. 
Lupescu, N., S. Arad, S. Geresh, M. A. Bernstein and R. Glaser (1991). Structure of some sulfated sugars isolated after acid hydrolysis of the extracellular polysaccharide of Porphyridium sp., a unicellular red alga. Carbo. Res. 210: 349-352.

McIntyre, D. D., C. Ho and H. J. Vogel (1990). One-dimensional nuclear magnetic resonance studies of starch and starch products. Starch 7: 260-267.

McIntyre, D. D. and H. J. Vogel (1990). Two-dimensional nuclear magnetic resonance studies of starch and starch products. Starch 42(8): 287-293.

Mehta, V. B. and B. S. Vaidya (1978). Cellular and extracellular polysaccharides of the blue-green algae Nostoc. J. Exp. Bot. : 1423-1430.

Morris, I. (1981). Photosynthetic products, physiological state, and phytoplankton growth. Physiological Basis of Phytoplankton Ecology. Canadian Bull. Fish. Aqat. Sci. $83-102$.

Morris, I., H. E. Glover and C. S. Yentsch (1974). Products of photosynthesis by marine phytoplankton: the effect of environmental factors on relative rates of protein synthesis. Mar. Biol. 27: 1-9.

Mykelstad, S. (1977). Production of carbohydrates by marine planktonic diatoms. II. Influence of the N/P ratio in the growth and medium on the assimilation ratio, growth rate, and production of cellular and extracellular carbohydrates by Chaetoceros affinis var Willei (Gran) Hustedt and Skeletonema costatum (Grev.) Cleve. J. exp. mar. Biol. Ecol. 29: 161-179.

Myklestad, S., A. Haug and B. Larsen (1972). Production of carbohydrates by the marine diatom Chaetoceros Affinis var Willei (Gran) Hustedt. II. Preliminary investigation of the extracellular polysaccharide. J. exp. mar. Biol. Ecol. 9: 137-144.

Myklestad, S., O. Holm-Hansen, K. M. Varum and B. E. Volcani (1989). Rate of release of extracellular amino acids and carbohydrates from the marine diatom Chaetoceros affinis. J. Plankton Res. 11(4): 763-773.

Nevo, Z. and N. Sharon (1969). The cell wall of Peridinium westii, a non cellulosic glucan. Biochem. Biophys. Acta 173: 161-175.

Painter, T. J. (1983). Algal Polysaccharides. The Polysaccharides. Academic Press. 195285.

Parsons, T. R., K. Stephens and J. D. H. Strickland (1961). On the chemical composition of eleven species of marine phytoplankton. J. Fish. Res. Bd. Canada 18(6): 1001-1016.

Paulsen, B. S. and S. Myklestad (1978). Structural studies of the reserve glucan produced by the marine diatom Skeletonema costatum (grev.) Cleve. Carbo. Res. 62: 386-388.

Percival, E., M. A. Rahman and H. Weigel (1980). Chemistry of the polysaccharides of the diatom Coscinodiscus nobilis. Phytochem. 19: 809-811.

Schmidt, W., G. Drews, J. Weckesser, I. Fromme and D. Borowiak (1980).

Characterization of the lipopolysaccharides from eight strains of the cyanobacterium Synechococcus. Arch. Microbiol. 127: 209-215. 
Shivley, J. M. (1988). Inclusions: granules of polyglucose, polyphosphate, and poly- $\beta$ hydroxybutyrate. Meth. Enzymol. 167: 195-203.

Smestad, B., A. Haug and A. Myklestad (1974). Production of carbohydrates by the Marine Diatom Chaetoceros affinis var. Willei (Gran) Hustedt. III. Structural studies of the extracellular polysaccharide. Acta Chem. Scand. 28: 662-666.

Smestad, B., A. Haug and S. Myklestad (1975). Structural studies of the extracellular polysaccharide produced by the diatom Chaetoceros curvisetus Cleve. Acta Chem. Scand. B 29: 337-340.

Smith, R. E. H., P. Clement, G. F. Cota and W. K. W. Li (1987). Intracellular photosynthate allcation and the control of arctic marine ice algal production. J. Phycol. 23: 124-132.

Smucker, R. A. and R. Dawson (1986). Products of photosynthesis by marine phytoplankton: chitin in TCA "protein" precipitates. J. Exp. Mar. Biol. Ecol. 104: 143152.

Sundh, I. (1989). Characterization of phytoplankton extracellular products (PDOC) and their subsequent uptake by heterotrophic organisms in a mesotrophic forest lake. $J$. Plankton Res. 11(3): 463-486.

Vaidya, B. S. and V. Mehta (1989). Carbohydrates in some blue green algae. Phykos 28: $89-100$.

Varum, K. M., J. K. Bjarne and S. Myklestad (1986). Structure of a food-reserve $\beta$-Dglucan produced by the haptophyte alga Emiliania huxleyi (Lohmann) Hay and Mohler. Carbo. Res. 152: 243-248.

Vieira, A. A. H. and S. Myklestad (1986). Production of extracellular carbohydrate in cultures of Ankistrodesmus densus Kors. (Chlorophyceae). J. Plankton Res. 8(5): 985994.

Weber, M. and G. Wober (1975). The fine structure of the branched $\alpha$-glucan from the blue-green alga Anacystis nidulans: comparison with other bacterial glycogens and phytoglycogen. Carbo. Res. 39: 295-302.

Weckesser, J. and U. J. Jurgens (1988). Cell walls and external layers. Methods in Enzymology 167: 173-188.

Zelibor, J. L., jr., L. Romankew, P. G. Hatcher and R. R. Colwell (1988). Comparative analysis of the chemical composition of mixed and pure cultures of green algae and their decomposed residues by $13 \mathrm{C}$ nuclear magnetic resonance spectroscopy. Appl. Environ. Microbiol. 54(4): 1051-1060. 
136 


\section{7}

\section{Chapter Four \\ Effect of Chemical Structure on Carbohydrate Degradation by Anaerobic Marine Bacteria: Degradation of Di- and Oligosaccharides}

\section{Introduction}

As discussed in Chapter 1, the cycling of organic matter in anoxic marine sediments is dependent upon an interplay of chemical and microbial processes. A combination of microbiological and geochemical approaches is used here to provide new insights into the factors controlling the degradation of macromolecular organic matter. A first step in this type of investigation was to establish model systems with which to study bacterial degradation of carbohydrates. The fundamental requirements were a reproducible experimental system in which substrate, intermediates, and products could be characterized, and a natural mixed microbial population which approximated the degradative capabilities of microorganisms in marine sediments. Model systems were well suited for this study because the carbon source could be controlled, so carbon transformations and recovery could be determined quantitatively. In addition, multiple replicates could be made to check reproducibility of the cultures with every substrate in every culture series, as well as between culture series over time periods of months to years.

A series of structurally related di- and oligosaccharides were selected as substrates for the first several series of culture experiments because the degradation of these carbohydrates has not apparently been studied in marine systems, even though lowmolecular weight carbohydrates, including some of the substrates used in the culture series, have been identified in seawater (Sakugawa and Handa, 1985). The bacterial metabolism of glucose and other monosaccharides is well understood (Wood, 1961; Gottschalk, 1986; Clark, 1989), and fermentation of glucose disaccharides has been studied in pure cultures of bacteria (Weimer and Zeikus, 1977; Bernier and Stutzenberger, 1987). Glucose uptake by bacteria in seawater (Vaccaro and Jannasch, 1966; Takahashi and Ichimura, 1971; Gocke et al., 1981, for example) and sediments (Meyer-Reil, 1978) has also been investigated. Uptake of specific low molecular weight carbohydrates other than glucose, however, has apparently not been been investigated in marine systems. In studies of the microbial populations of seawater and sediments, glucose uptake has been used as a proxy for carbohydrate metabolism in general, even though the ability to ferment different monosaccharides is variable among different species of bacteria. One of the characteristics commonly used to differentiate pure cultures of bacteria from one another, in fact, is their ability to metabolize specific hexoses or pentoses. In addition, while many bacteria can 
metabolize glucose, the ability to utilize glucose polysaccharides such as cellulose is restricted to select groups of bacteria, so glucose metabolism does not necessarily reflect metabolism even of glucose oligosaccharides.

Bacterial degradation of carbohydrate-containing material in marine systems has been investigated in studies focusing on the bacterial utilization of phytoplankton exudates (Newell et al., 1981; Cole et al., 1982; Iturriaga and Zsolnay, 1983; Biddanda, 1988, for example). A number of studies have shown that the phytoplankton exudates contain carbohydrates (Burney, 1986), including high molecular weight polysaccharides (Khailov, 1968; Hama and Handa, 1987; Sundh, 1989). These carbohydrates have not been well characterized, and little structural information other than nominal molecular weight range was determined for the phytoplankton exudates.

The goal of culture studies described in this chapter was to establish a set of reproducible model systems which could be used to compare the degradation of a series of structurally related di- and oligosaccharides. Because disaccharides are well within the molecular weight range which can be directly taken up without extracellular hydrolysis by bacteria, the degradation of these substrates corresponds to the dissolved monomers $\rightarrow$ total $\mathrm{CO}_{2}$ transition $(\mathrm{F})$ of Fig. 1.1. Variations in degradation rates or patterns for different disaccharides must be due to the influence of specific structural features on the ability of a diverse range of bacteria to take up or intracellularly degrade the disaccharides. Studying the degradation of small, directly-ingestible carbohydrates therefore also provided a reference for the polysaccharide degradation experiments presented in the next chapter. Transformation of dissolved polymers to $\mathrm{CO}_{2}$ involves at least two major transitions, represented by $\mathrm{E}$ and $\mathrm{F}$ in Fig. 1.1. By comparing the degradation of carbohydrates which have the same chemical structure and differ only in size, effects due to the requirement for extracellular enzymatic hydrolysis of a large polysaccharide $(\mathrm{F})$ can be distinguished from effects which are attributable to bacterial transport and internal degradation of a smaller carbohydrate with the same structure (E).

This chapter presents the results of a series of experiments in which replicate cultures of anaerobic bacteria from sulfate-reducing sediments were enriched on a suite of structurally related substrates. The disappearance of substrate was measured using high pressure liquid chromatography (HPLC), the evolution of gaseous end products $\left(\mathrm{CO}_{2}\right.$, $\mathrm{CH}_{4}, \mathrm{H}_{2} \mathrm{~S}$ ) was monitored with gas chromatography, and carbon balance was determined at the end of each culture. 


\section{Methods}

In order to compare the degradation of several structurally-related substrates in parallel cultures, a culture system which was reproducible and relatively simple was needed. Hungate anaerobic techniques (Hungate, 1969) were used to prepare replicate $120 \mathrm{~mL}$ serum vials with $50 \mathrm{~mL}$ artificial seawater (Table 4.1) under a $\mathrm{N}_{2}$ headspace. The vials were sealed with $10 \mathrm{~mm}$ butyl rubber stoppers and aluminum crimp-seals. After addition of substrate and inoculum (described below), each series of culture vials was incubated in the dark at $25^{\circ} \mathrm{C}$ for the course of the experiment (20-25 days). A flow diagram of the culture experimental procedures is shown in Fig. 4.1.

Five series of experiments were conducted over a 9 month time period. Two to six substrates were selected for each series, and most substrates were used in at least two separate culture series in order to check reproducibility of results between culture series. In every case, a single substrate was added to an individual vial. All of the substrates and all of the blanks (described below) were run in triplicate in every culture series.

\section{Substrates}

In order to directly compare the effects of specific differences in substrate composition, linkage position, and linkage orientation, eight disaccharides composed of monomers commonly observed in marine environments (Cowie and Hedges, 1984; Hamilton and Hedges, 1988) and a small size-range of maltooligosaccharides (Fig. 4.2) were selected as substrates for the degradation experiments. Four of the substrates were homodisaccharides, in which two glucose units were linked together, and four were heterodisaccharides of glucose-galactose, galactose-arabinose, or galactose-mannose. A number of features, including monomer composition, linkage position, and linkage orientation can be directly compared with this set of substrates. Cellobiose and lactose differ from one another in monomer composition; the former is a $\beta(1,4)$-linked glucose disaccharide, while the latter is a galactose-glucose disaccharide with a $\beta(1,4)$ linkage (Fig. 4.2). Similarly, isomaltose is an $\alpha(1,6)$ glucose disaccharide, while melibiose is an $\alpha(1,6)$ galactose-glucose disaccharide. Lactose and GLM (galactose- $\beta(1,4)$-mannose) both are $\beta(1,4)$ linked disaccharides, composed of galactose-glucose and galactose-mannose, respectively. The pairs of disaccharides maltose-isomaltose and cellobiose-gentiobiose differ in linkage position, with $(1,4)$ and $(1,6)$ linkages. Anomeric linkage ( $\alpha$ vs. $\beta$ ) differentiate maltose and cellobiose, and isomaltose and gentiobiose. GLA (galactose- $\beta(1,3)$-arabinose) differs from the other substrates both in linkage position and composition, since it is the only 


$\begin{array}{lll}\mathrm{NaCl} & 250 \mathrm{mM} & \\ \mathrm{MgSO}_{4} \cdot 7 \mathrm{H}_{2} \mathrm{O} & 30 \mathrm{mM} & \\ \mathrm{MgCl}_{2} \cdot 6 \mathrm{H}_{2} \mathrm{O} & 20 \mathrm{mM} & \\ \mathrm{CaCl}_{2} \cdot 2 \mathrm{H}_{2} \mathrm{O} & 10 \mathrm{mM} & \\ \mathrm{KCl} & 10 \mathrm{mM} & \\ \mathrm{NH}_{4} \mathrm{Cl} & 19 \mathrm{mM} & \\ \mathrm{K}_{2} \mathrm{HPO} & \\ & 0.33 \mathrm{mM} & \\ \text { HEPES } & 15 \mathrm{mM} & \\ & & \\ \text { resazurin } & 1 \mathrm{~mL} \text { stock solution }(0.1 \%) & 100 \mathrm{mM} \\ & & 0.25 \mathrm{mM} \\ \text { trace metals } & 1 \mathrm{~mL} \text { stock solution: } & 0.8 \mathrm{mM} \\ & \mathrm{HCl} & 0.3 \mathrm{mM} \\ & \mathrm{MnCl}_{2} \cdot 4 \mathrm{H}_{2} \mathrm{O} & 0.1 \mathrm{mM} \\ & \mathrm{CoCl}_{2} \cdot 6 \mathrm{H}_{2} \mathrm{O} & 0.1 \mathrm{mM} \\ & \mathrm{ZnSO}_{4} \cdot 7 \mathrm{H}_{2} \mathrm{O} & 0.01 \mathrm{mM} \\ & \mathrm{H}_{3} \mathrm{BO}_{3} & 0.08 \mathrm{mM} \\ & \mathrm{NiCl}_{2} \cdot 6 \mathrm{H}_{2} \mathrm{O} & 1.0 \mathrm{~g} / \mathrm{L}\end{array}$

$\mathrm{Na}_{2} \mathrm{~S} \quad 400 \mu \mathrm{M}$

Table 4.1

Artificial seawater medium used for all cultures. Ingredients (except the $\mathrm{Na}_{2} \mathrm{~S}$ ) were mixed in $\mathrm{Q}-\mathrm{H}_{2} \mathrm{O}$, and then all further steps were carried out under $\mathrm{N}_{2}$, using Hungate anaerobic techniques. The medium was boiled vigorously for approximately 4 minutes, then was quickly cooled on an ice slurry, and the $\mathrm{Na}_{2} \mathrm{~S}$ solution was added to the medium. Medium was dispensed in $50 \mathrm{~mL}$ portions into $120 \mathrm{~mL}$ serum vials, which were capped with $1 \mathrm{~cm}$ butyl rubber stoppers, crimp sealed, and autoclaved. Any vials in which the medium showed any pink coloration upon removal from the autoclave (i.e., incomplete reduction/ presence of oxygen) were discarded. 


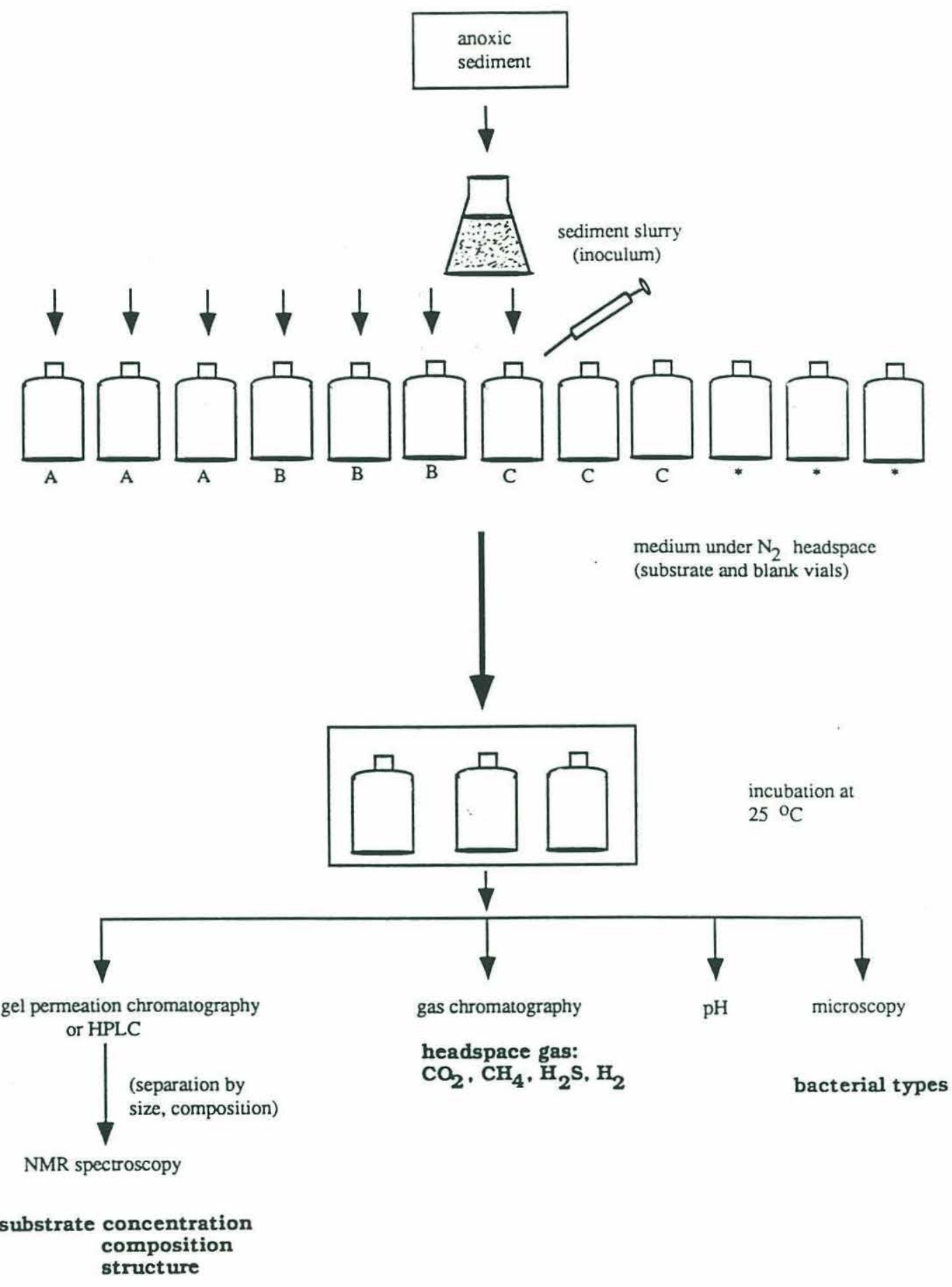

Fig. 4.1

Flow diagram of culture experimental procedures. All culture procedures carried out under nitrogen, as described in the text and the caption of Table 4.1. 


\section{2}
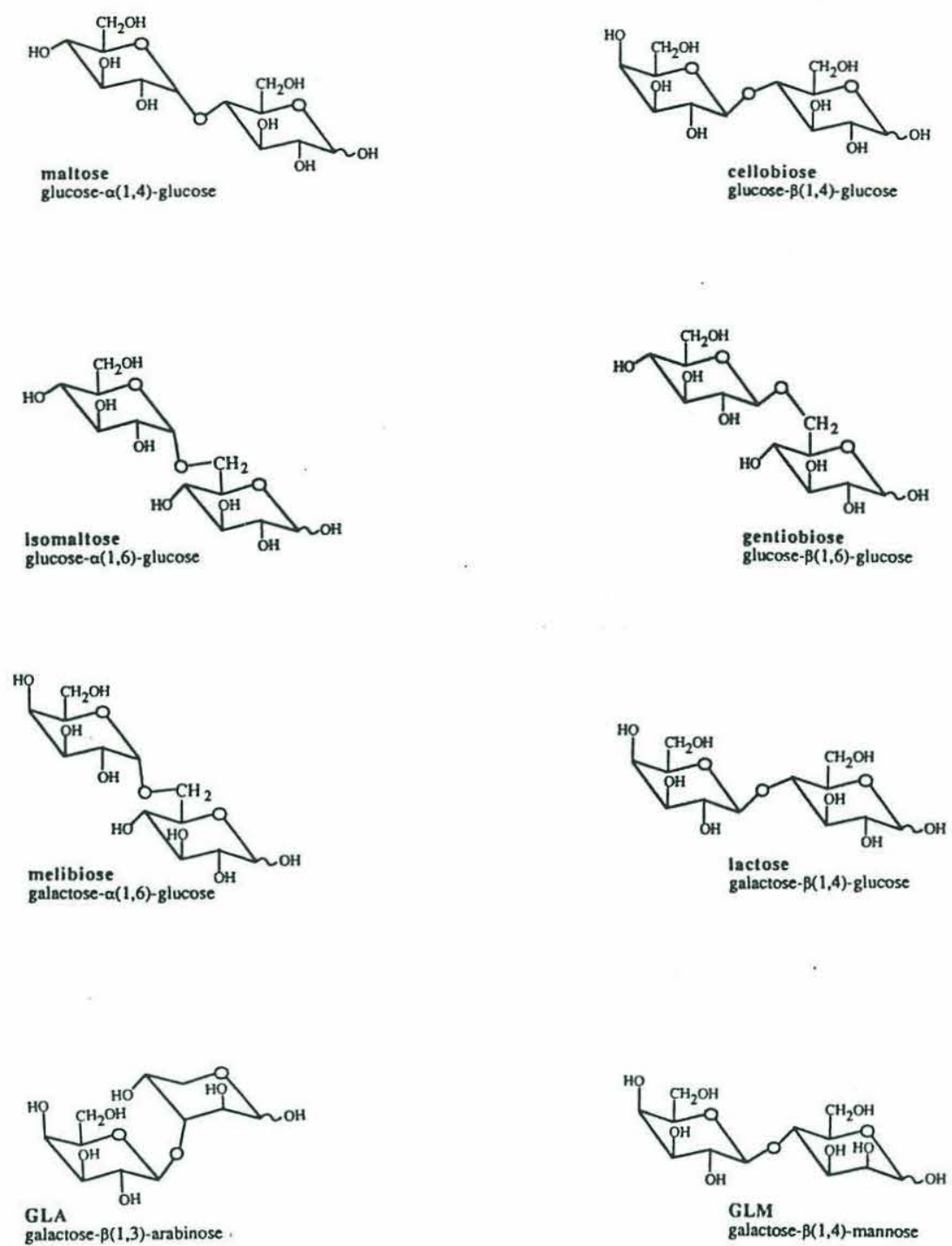

maltooligosaccharides
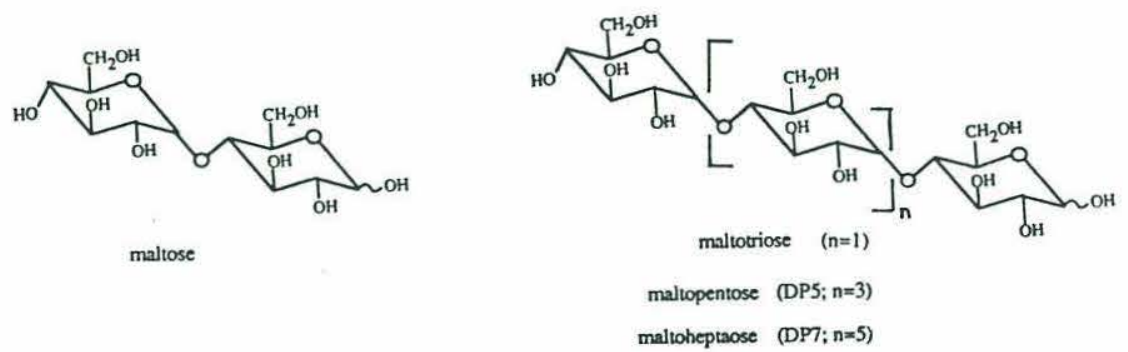

Fig. 4.2

Structures of di- and oligosaccharide substrates. 
(1,3)-linked disaccharide, as well as the only disaccharide which includes a five-carbon sugar, arabinose. The maltooligosaccharide series, maltose, maltotriose, maltopentaose, and maltoheptaose, differ from each other only in size, since all are composed of $\alpha(1,4)$ linked glucose units.

\section{Preparation of Substrates and Inoculum}

All substrates were used as received from Sigma Chemical Co. After the culture vials had been prepared and autoclaved (see legend, Table 4.1), the substrates were dissolved in deoxygenated water under $\mathrm{N}_{2}$, filter sterilized, and added to each vial. The final substrate concentration (after addition of inoculum slurry) was $1 \mathrm{mM}$. The inoculum source was sulfate-reducing, anoxic surface sediment (depth ca. 5 m), collected during October 1990 from Salt Pond (Woods Hole, MA), a highly productive marine glacial basin (Lee, 1992)). The bottom water and sediments are anoxic; the mud consisted of very fine-grained black sediments. Small $(0.5 \mathrm{~L})$ jars were filled to the top with mud and stored at $4{ }^{\circ} \mathrm{C}$. The inoculum was prepared by adding (under nitrogen) ca. $27 \mathrm{~cm}^{3}$ sediment to $70 \mathrm{~mL}$ of oxygen-free deionized (Millipore "Q" system) water. The slurry was continuously mixed on a magnetic stirring plate, and $1.5 \mathrm{~mL}$ was withdrawn by syringe and added to each vial. Total sediment addition to a vial typically ranged from $80-100 \mathrm{mg}$ dry weight, which corresponds to approximately $150-250 \mu \mathrm{mol}$ carbon (see below); no systematic differences in culture behavior with inoculum mass were observed for any culture. Total volume of each culture was $52 \mathrm{~mL}$ ( $50 \mathrm{~mL}$ medium, $0.5 \mathrm{~mL}$ substrate solution, and $1.5 \mathrm{~mL}$ inoculum slurry.)

\section{Culture Sampling}

For every culture series, each vial was subsampled for headspace gas composition and concentration, $\mathrm{pH}$, and substrate concentration on a daily basis for the first 15 days; further samples were taken at 20 to 25 days. Visual examinations of the cultures by phase-contrast microscope were made on a periodic basis, and total $\mathrm{CO}_{2}$ was determined three to four times during the course of each experiment.

\section{Headspace Gas Measurement}

Gas analyses were made by isothermal gas chromatography (GC; Shimadzu GC-8A), using a thermal conductivity detector (TCD), dual custom columns (Hayesep Q, 100/120 
mesh, 8' teflon-coated stainless steel; Alltech) and a glass-lined injection port. The carrier gas was ultra high-purity $\mathrm{N}_{2}$ at a flow rate of $50 \mathrm{~mL} / \mathrm{min}$. Inlet and detector temperature was $70^{\circ} \mathrm{C}$, column temperature was $50^{\circ} \mathrm{C}$, and TCD current was $90 \mathrm{~mA}$. Signal output was stored and processed with a Vista Varian 402 integrator. The GC system was calibrated with two gas standards consisting of $100 \% \mathrm{CH}_{4}$, and of $5 \% \mathrm{CO}_{2}$ and $10 \% \mathrm{H}_{2}$ in $\mathrm{N}_{2}$. Injection volume was $250 \mu \mathrm{L}$; detection limits were 180,130 , and $2 \mathrm{ppm}$ by volume for $\mathrm{CO}_{2}, \mathrm{CH}_{4}$, and $\mathrm{H}_{2}$, respectively. Hydrogen sulfide was also detected both in headspace gases and in acidified medium (measured for total $\mathrm{CO}_{2}$ determinations; see below), but was not quantified rigorously because of reactivity witha section of stainless steel tubing connecting the teflon-lined column with the TCD. Instrumental standard deviation for replicate measurements of a standard gas mixture was $4 \%$.

\section{Substrate Quantification}

One $\mathrm{mL}$ medium was removed from each vial daily for the first 15 days to determine substrate concentration. The $\mathrm{pH}$ was measured, and the medium was filtered to remove large particles and stored in a clean vial at $-40^{\circ} \mathrm{C}$ for later analysis. The samples were prepared for carbohydrate analysis by bringing them to room temperature, and removing $500 \mu \mathrm{l}$ from each vial. To partially desalt the sample, the medium was passed through a pre-rinsed "On-Guard" Ag column (Dionex Corporation) and collected along with a 2500 $\mu \mathrm{L} \mathrm{Q}$-water wash. The mini-column was rinsed with $2500 \mu \mathrm{L} \mathrm{Q}$-water between samples. Approximately six samples ( $3 \mathrm{~mL}$ medium) could be desalted per cartridge; no carryover was observed between samples. The $3000 \mu \mathrm{L}$ (sample + rinse) were reconcentrated by placing the vial in a warm sand bath and evaporating the sample to dryness under a stream of nitrogen. The samples were redissolved in $500 \mu \mathrm{L} \mathrm{Q}$-water prior to analysis. Tests with standards demonstrated that carbohydrate recovery from the desalting and evaporation procedure was $100 \%$.

The samples were analyzed for carbohydrates using a high-pressure liquid chromatography (HPLC) system consisting of two $15 \mathrm{~cm}, 3 \mu \mathrm{NH}_{2}$-bonded Si cartridges (Alltech) connected in series and an evaporative light-scattering detector (ELSD; Varex Corporation). The mobile phase was $65: 35$ acetonitrile:water at $1 \mathrm{~mL} / \mathrm{min}$. With this mobile phase, DP1-DP6 oligosaccharides (for example, glucose-maltohexaose) are completely separated, although the DP5-DP6 peaks are very broad (Fig. 4.3). To improve peak resolution, maltopentose and maltohepatose were analyzed with a 50:50 acetonitrile:water mobile phase. If any smaller oligosaccharides had been present in the medium as intermediates from the degradation of the larger maltooligosaccharides (see 


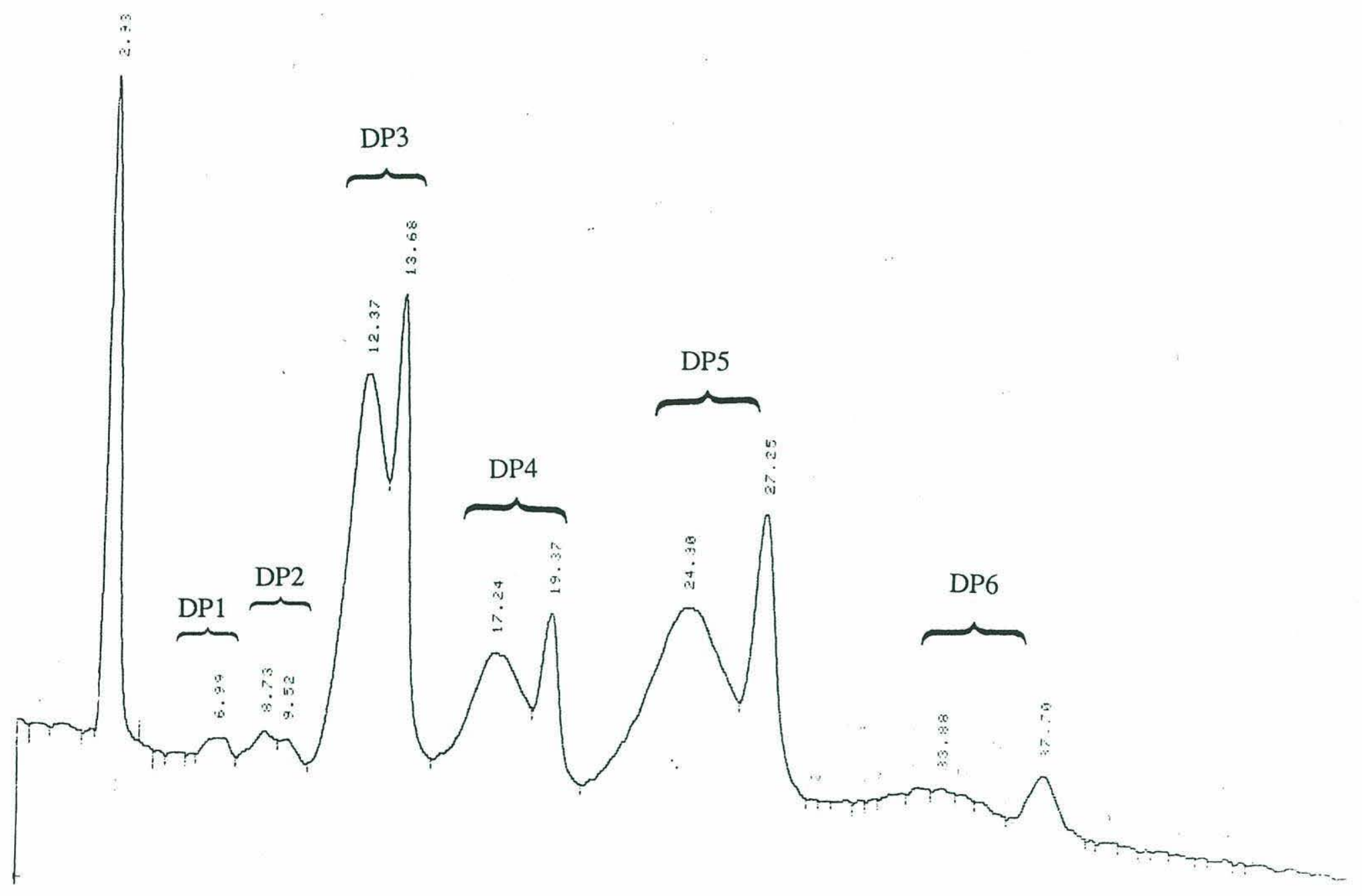

Fig. 4.3

Separation of DP1-6 maltooligosaccharides using HPLC (see text for details). 
below), they would still have been resolved under these conditions. Monosaccharides (glucose, galactose, mannose, fucose, ribose, rhamnose, arabinose, and xylose) can also be separated with this system, using an 87:13 acetonitrile:water mobile phase at a flow rate of $0.8 \mathrm{~mL} / \mathrm{min}$., although glucose and galactose, and ribose and rhamnose, are not baseline-resolved. The ELSD was run at a temperature of $150^{\circ} \mathrm{C}$ with a nitrogen flow rate of $50 \mathrm{~mL} / \mathrm{min}$ and a time constant of 5 seconds. Data was acquired with a Hewlett-Packard 3396 II integrator. Standard deviation of replicate analyses was $6 \%$. Initial substrate concentration per $100 \mu \mathrm{L}$ injection was approximately $35 \mu \mathrm{g}$; the detection limit of the system is approximately $1 \mu \mathrm{g}$ carbohydrate.

\section{Total $\mathrm{CO}_{2}$ Determination}

Total $\mathrm{CO}_{2}$ was measured three to four times during each culture series by separately determining $\mathrm{CO}_{2}$ in the medium, and adding this quantity to the headspace $\mathrm{CO}_{2}$ which was measured on a daily basis. To determine $\mathrm{CO}_{2}$ in the medium, $1 \mathrm{~mL}$ medium was withdrawn from each vial (and replaced by $1 \mathrm{~mL}$ of nitrogen) and injected into a nitrogenfilled, sealed $10 \mathrm{~mL}$ serum vial. The medium was acidified to approximately $\mathrm{pH} 1$ by addition of concentrated $\mathrm{HCl}$, and shaken for 15 minutes on an orbital shaking table before $250 \mu \mathrm{L}$ of headspace were removed with a gas tight syringe for GC analysis. Standard deviation of replicate measurements of $\mathrm{CO}_{2}$ in the medium was $6 \%$.

\section{Blanks}

Addition of carbon other than substrate to the cultures was minimized in order to ensure that the carbohydrate substrate was the sole carbon source for bacterial growth. The artifical seawater medium in each culture contained $0.154 \mu \mathrm{mol}$ carbon in the form of Sequestrine (Ciba-Geigy; a chelated iron complex necessary to prevent precipitation of iron upon autoclaving). Resazurin (a redox indicator), and HEPES buffer added an additional 258 $\mu \mathrm{mol}$ carbon. Measurements of Salt Pond sediments collected at the same site in autumn of 1992 showed the average organic carbon content was $\sim 1800 \mu \mathrm{M} / \mathrm{cm}^{3}$ (pers. comm., Dr. Brian Howes). Approximating from inoculation volume and the quantity of sediment remaining in the inoculum slurry after culture inoculation, 150-250 $\mu$ mol additional carbon could potentially be available to the bacteria. For all disaccharides except GLA (which as a hexamer-pentamer disaccharide contains only 11 , not 12 , carbons), substrate concentration was equivalent to $624 \mu \mathrm{mol}$ carbon (GLA concentration was equivalent to $572 \mu \mathrm{mol}$ carbon). Substrate carbon therefore comprised $55-60 \%$ of the total carbon in the medium, 
including carbon in the buffer and inoculum slurry. In order to check that the bacteria were not utilizing the buffer or substrates which might be present in the inoculum slurry, a series of blanks were included in each culture series. The blanks consisted of vials with substrate which were not inoculated ("B vials"), vials without substrate which were inoculated ("I vials"), and vials which were inoculated and autoclaved before substrate was added ("IAS" vials). All three types of blanks showed only a low background level of $\mathrm{CO}_{2}(<5$ $\mu \mathrm{mol}$ ) which was attributed to degassing of the $\mathrm{CO}_{2}$ naturally present in the water used to make the medium. The substrate levels in the B and IAS vials were identical on the first and last days of the culture experiments, demonstrating that loss of substrate was not due to sorption to the vial or sediment particles.

\section{Results and Discussion}

General Results

In almost all culture experiments, substrates were consumed within 2-4 days of inoculation. Fig. 4.4 shows a typical profile (maltose in this example) of substrate disappearance and evolution of gases in the headspace. Substrate concentration decreased slightly on day 1 , and was below the limit of detection on day 2. Headspace $\mathrm{CO}_{2}$ levels rose sharply between day 2 and 6 , increased slowly until day 12 , increased at a faster rate between days 13 and 15, and reached a final level of $97 \mu \mathrm{mol}$ on day 25 . Hydrogen was detected in the headspace on day 1 , increased in concentration through day 3 , and then decreased abruptly and was below detection limit by day 5 . Low levels of methane, which persisted through the course of the experiment, were first detected on day 4. Hydrogen sulfide (not shown in Fig. 4.4) was first detected in the headspace on day 4, and steadily increased in concentration with time. Recovery of substrate carbon as total $\mathrm{CO}_{2}$ at the end of the culture series ( 20 or 25 days) typically was between $60 \%$ and $70 \%$ of the theoretical maximum (i.e., the amount which would be present if $100 \%$ of the substrate were converted to $\mathrm{CO}_{2}$ ). Approximately $0.25 \%$ of the total carbon was converted to $\mathrm{CH}_{4}$.

The pattern of swift substrate consumption, followed by a several-step increase in $\mathrm{CO}_{2}$, conforms to a sequence of degradation steps which would be expected from an anaerobic bacterial consortium. Disaccharides are within the size range which bacteria can directly ingest, so uptake is not limited by the need for initial extracellular hydrolysis of substrate. Pure culture studies have demonstrated that bacteria can swifly consume disaccharides; cultures of Thermomonospora curvata, for example, reduced cellobiose levels from $9 \mathrm{mM}$ to $1 \mathrm{mM}$ over a timespan of 23 hours (Bernier and Stutzenberger, 1987). 


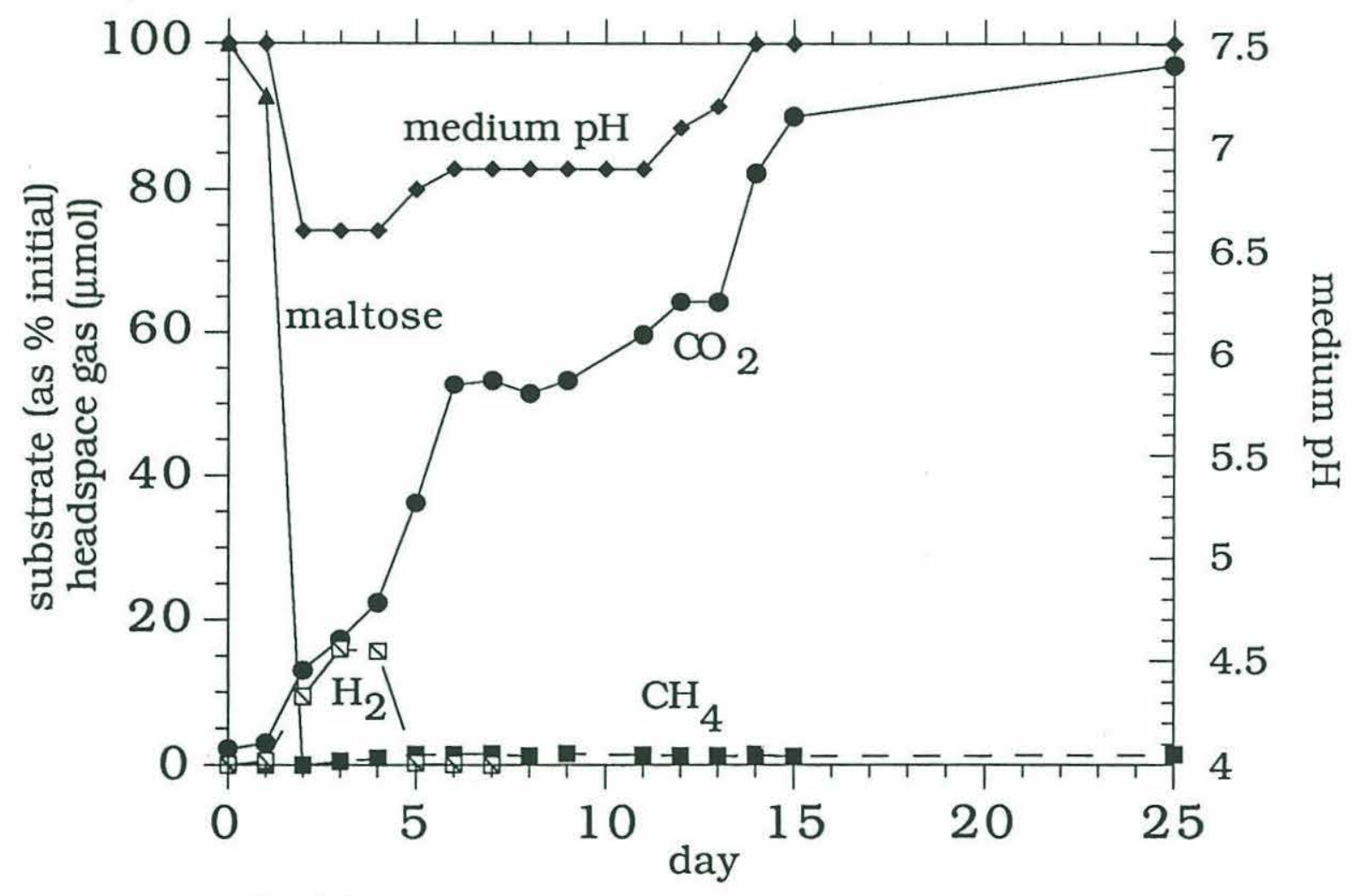

Fig. 4.4

Degradation of maltose and evolution of headspace gases. Changes in $\mathrm{pH}$ over the course of the culture are also shown (right axis). 
The pattern of $\mathrm{CO}_{2}$ evolution is also consistent with the substrate consumption pattern. Carbon dioxide is one of several products of the initial stages of carbohydrate fermentation by a culture of bacteria (Wood, 1961; Gottschalk, 1986). Anaerobic bacteria which ferment carbohydrates typically assimilate approximately $10 \%$ of the substrate carbon (Clark, 1989), and excrete most of the remainder in the form of low molecular weight organic compounds such as short-chain acids, alcohols, and $\mathrm{CO}_{2}$. The acids and alcohols are taken up by other bacteria in the community, which can further utilize them, ultimately oxidizing much of the carbon to $\mathrm{CO}_{2}$. The initial increase in $\mathrm{CO}_{2}$ observed in the cultures most likely corresponds to $\mathrm{CO}_{2}$ produced directly from substrate fermentation. The 'plateau' in headspace $\mathrm{CO}_{2}$ between days 6 and 11 probably represents the time required by the next group of bacteria to double to sufficient numbers to convert the short-chain acids and alcohols produced in the initial fermentation to $\mathrm{CO}_{2}$. Initial bacterial numbers, bacterial doubling time, as well as rate of substrate conversion, will determine the amount of time required to metabolize a specific substrate.

The transient presence of small organic acids as secondary metabolites in the cultures was also indicated by temporary changes in medium $\mathrm{pH}$. In all of the cultures, medium $\mathrm{pH}$ provided a subtle indication of changes in quantities of acidic fermentation products (presumably acetate, lactate, etc. in addition to $\mathrm{CO}_{2}$ ) in the culture medium. Substrate degradation was accompanied by a decline in medium $\mathrm{pH}$ of up to ca. $0.9 \mathrm{pH}$ units. (Note that this drop occurred in spite of the $15 \mathrm{mM}$ HEPES buffer in the medium; $\mathrm{pH}$ control had been a problem in initial test cultures with lower buffer and higher substrate concentrations.) The decline was presumably due principally to the production of small organic acids, and not to the presence of $\mathrm{CO}_{2}$, because as headspace $\mathrm{CO}_{2}$ levels increased between days 5 and $15, \mathrm{pH}$ also gradually increased. This is consistent with conversion of organic acids to $\mathrm{CO}_{2}$. The opposite effect would be expected (i.e., a decrease in headspace $\mathrm{CO}_{2}$ with an increase in $\mathrm{pH}$ ) if headspace $\mathrm{CO}_{2}$ levels were simply a function of carbonate equilibrium in a closed system. Biological production of $\mathrm{CO}_{2}$ from organic acids dominated changes in both $\mathrm{pH}$ and $\mathrm{CO}_{2}$ levels in these cultures. By the end of the culture series, the $\mathrm{pH}$ returned to its initial level, implying that organic acids produced early in the culture series had been completely consumed and converted to cellular carbon and $\mathrm{CO}_{2}$.

The sequential nature of these transformations is also indicated by the appearance and subsequent consumption of $\mathrm{H}_{2}$ in the headspace from day 2 to day $5: \mathrm{H}_{2}$ is a byproduct of carbohydrate fermentation, and is utilized by bacteria such as sulfate reducers and methanogens. The appearance of low levels of $\mathrm{CH}_{4}$ is reasonable in these model marine systems, since simultaneous high levels of sulfate reduction and low levels of 
methanogenesis have been observed in a wide range of marine sediments (Winfrey et al., 1981; Kuivila et al., 1990), and references therein.) Sulfate-reducing bacteria can outcompete methanogenic bacteria for substrates such as $\mathrm{H}_{2}$ and acetate, but methanogens can also utilize 'non-competitive' substrates such methanol, trimethylamine, and methionine (Oremland and Polcin, 1982). Methane typically was initially detected on day 4 or day 5 , and reached a maximum concentration of $1-2 \mu \mathrm{mol}$ by day 7 . The methanogens in the cultures could therefore be utilizing non-competitive substrates which were a byproduct of initial carbohydrate fermentation. Methane production could also be a function of bacterial growth rate. There are two major groups of sulfate reducers, one of which can only incompletely oxidize substrates such as lactate to acetate, and a second group which can oxidize substrates including acetate completely to $\mathrm{CO}_{2}$. Most acetate-utilizing sulfate reducers double much more slowly than the sulfate reducers which cannot utilize acetate (Widdel, 1988). If the methanogens double faster than the acetate-utilizing sulfate reducers, for example, they may be able to utilize acetate until the sulfate reducers reach sufficient numbers to control substrate utilization.

The melibiose cultures, in which $\mathrm{CH}_{4}$ levels usually reached $5-6 \mu \mathrm{mol}$ by day 7 , were the only exceptional case. The higher level of $\mathrm{CH}_{4}$ production was probably due to trace levels of methanol which may have been inadvertantly introduced with the substrate solution. Since melibiose is prepared commercially by recrystallizing from dilute solutions of methanol in water (pers. comm., U.S. Biochemicals), methanol may have taken the place of some of the waters of hydration in the melibiose crystals. There is no direct spectroscopic evidence, however, of methanol in the melibiose solutions. NMR spectra of melibiose were inconclusive, since the methanol $-\mathrm{CH}_{3}$ group has a chemical shift in the most crowded region of the melibiose spectrum. Comparison of spectra by subtraction was also attempted. The sample was evaporated (which would theoretically remove the methanol) and redissolved in $\mathrm{D}_{2} \mathrm{O}$, and a second spectrum was acquired under the same conditions. The results were again inconclusive; the low levels ( $<1 \%$ on a carbon basis) of methanol, if present, were not detectable against a high background signal level. Methanol contaminant could not be the source of methane in the cultures enriched on other substrates, however, since the other substrates such as lactose are commercially prepared by crystallization from pure water (pers. comm., U.S. Biochemicals).

\section{Reproducibility}

The headspace gas results show that this culture system fulfills a major prerequisite for comparative degradation experiments: results were highly reproducible, both within 
triplicate vials for each culture series, and between culture series run several months apart. Figs. 4.5 and 4.6 show headspace $\mathrm{CO}_{2}$ for lactose and maltose cultures during two different culture series: reproducibility between the triplicates of a series, as well as between the same substrate run in several series, was very good. The standard deviation of headspace $\mathrm{CO}_{2}$ measurements for an individual vial measured three times was $8 \%$, while standard deviation of three replicate vials in an individual culture series ranged from 8-25\% during the initial few days of the culture series, and was $7-14 \%$ for the later time points. Standard deviation of headspace $\mathrm{CO}_{2}$ levels in different culture series which included the same substrate (as shown in Figs. 4.4 and 4.5 ) was $10-25 \%$ for the first several days of the culture series, and $1-8 \%$ for the later time points. Relative changes in headspace $\mathrm{CO}_{2}$ levels were the greatest during the initial timepoints in a culture series, which accounts for the differences in standard deviation over the course of the series.

In addition to being reproducible, the cultures exhibited a sequence of transformations which are consistent with previous observations of anaerobic bacterial communities and consortia studies. Enrichment cultures of this type may serve as reasonable, simplified models of bacterial communities in anoxic sediments. This system could therefore be used to study the degradation of other substrates, such as proteins, lipids, or other types of complex organic matter. By eliminating many of the problems associated with matrix effects and uncharacterized and unquantified carbon sources, and by using a system simple enough to be run with multiple replicates, the effect of specific features of substrate structure can be directly compared, and bacterial transformation processes of these substrates can be studied in detail.

\section{Homo- and Heterodisaccharides}

The lactose and maltose headspace $\mathrm{CO}_{2}$ plots (Figs. 4.4 and 4.5) highlight a subtle difference between glucose homodisaccharide and heterodisaccharide substrates. As shown in Fig. 4.7, headspace $\mathrm{CO}_{2}$ increased very quickly for the glucose homodisaccharides (solid symbols), regardless of linkage orientation $(\alpha$ or $\beta$ ) or position $((1,4)$ or $(1,6)$ ) (isomaltose, which is not shown, yielded a profile similar to maltose). Headspace $\mathrm{CO}_{2}$ for the four heterodisaccharides (open symbols)—lactose, melibiose, GLM, and GLA-all showed at least a slight initial delay. In every case except lactose, the slight delay in evolution of headspace $\mathrm{CO}_{2}$ mirrored a slight delay in substrate degradation (Fig. 4.8). The most significant difference in substrate utilization was between cultures enriched on GLA and all of the other substrates, whether homo- or heterodisaccarides. Complete disappearance of GLA from the culture took 9 days, at least twice as long as for any other 


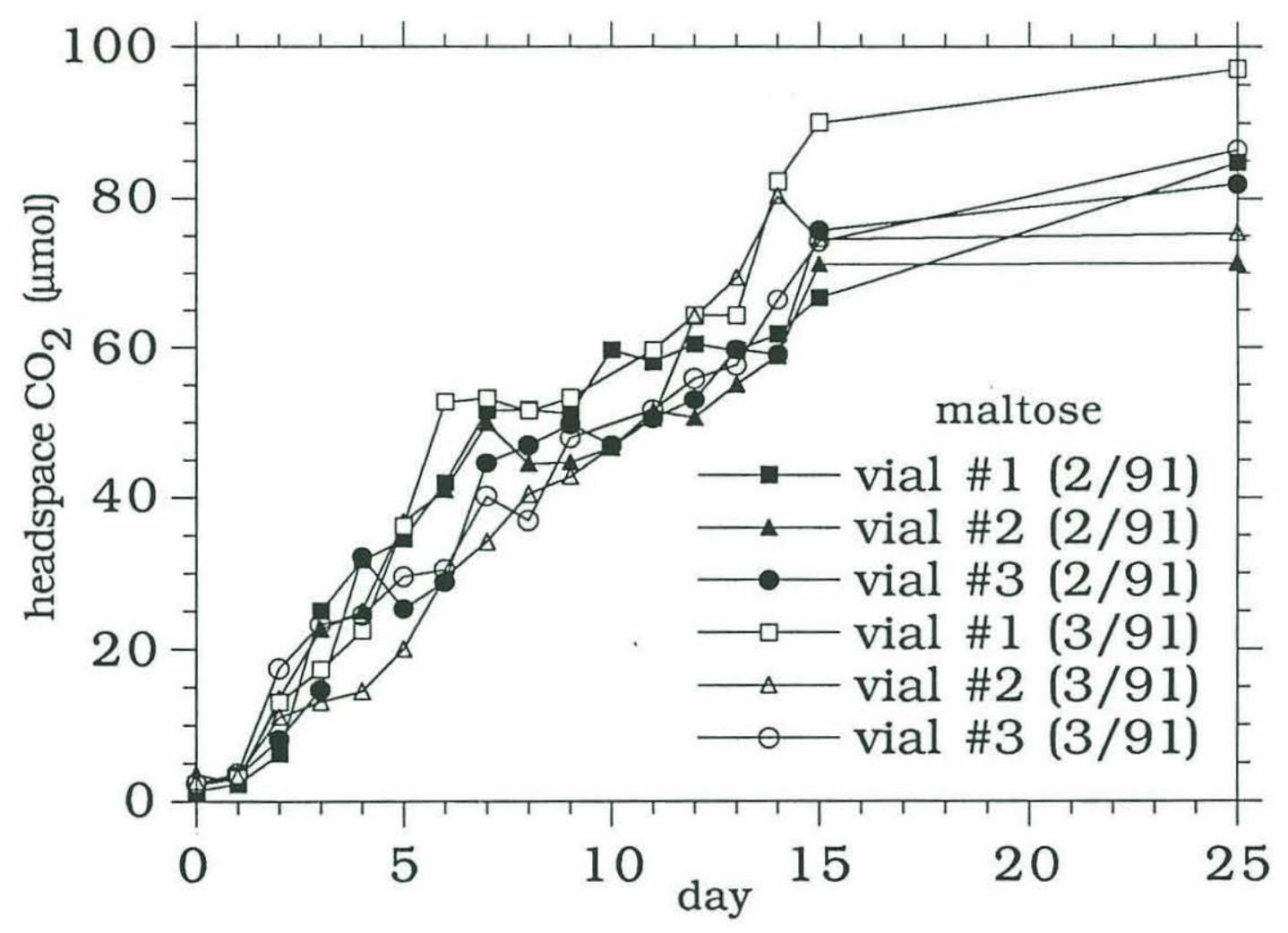

Fig. 4.5

Headspace $\mathrm{CO}_{2}$ profiles for maltose (triplicate vials from two culture series). 


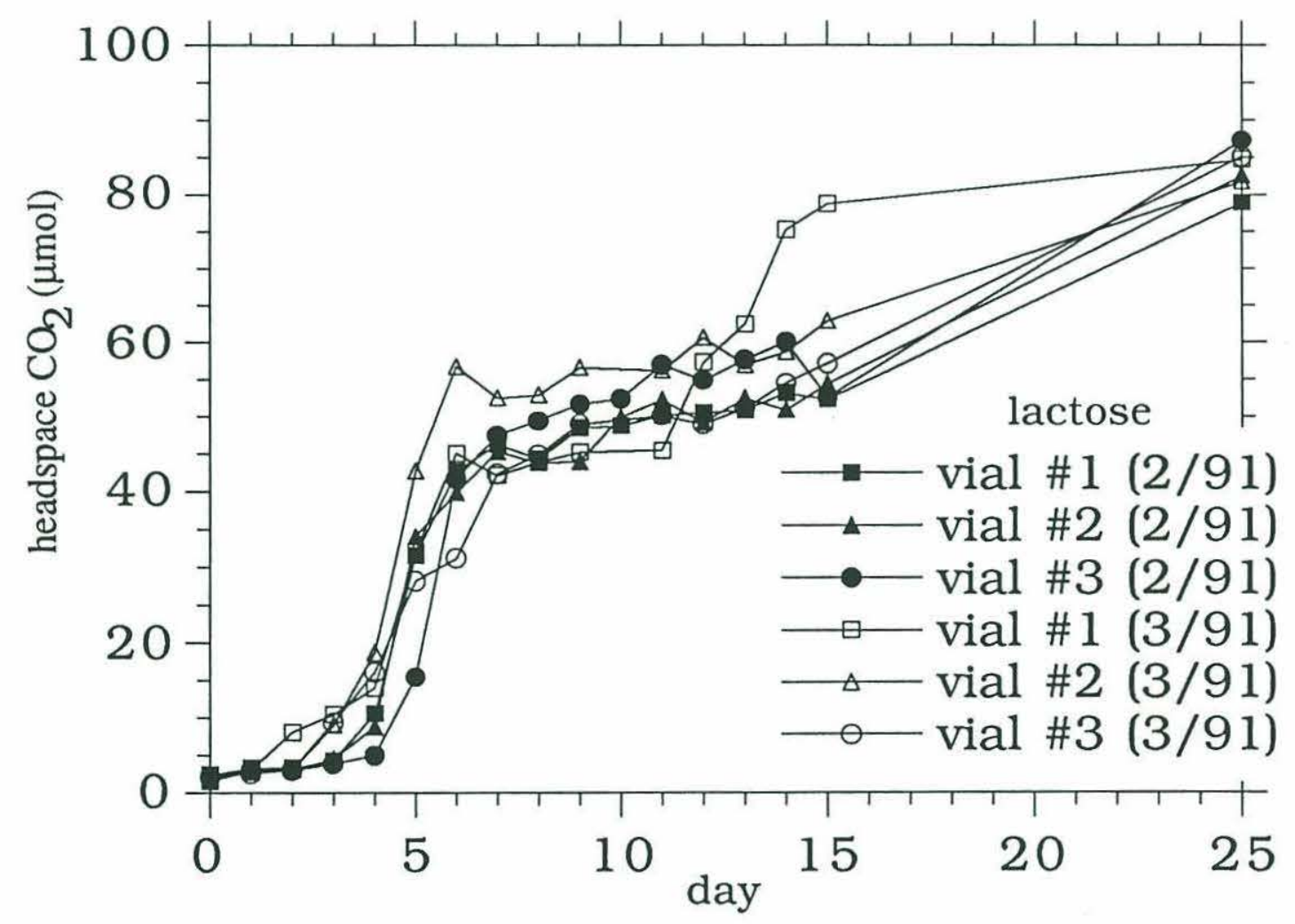

Fig. 4.6

Headspace $\mathrm{CO}_{2}$ profiles for lactose (triplicate vials from two culture series). 


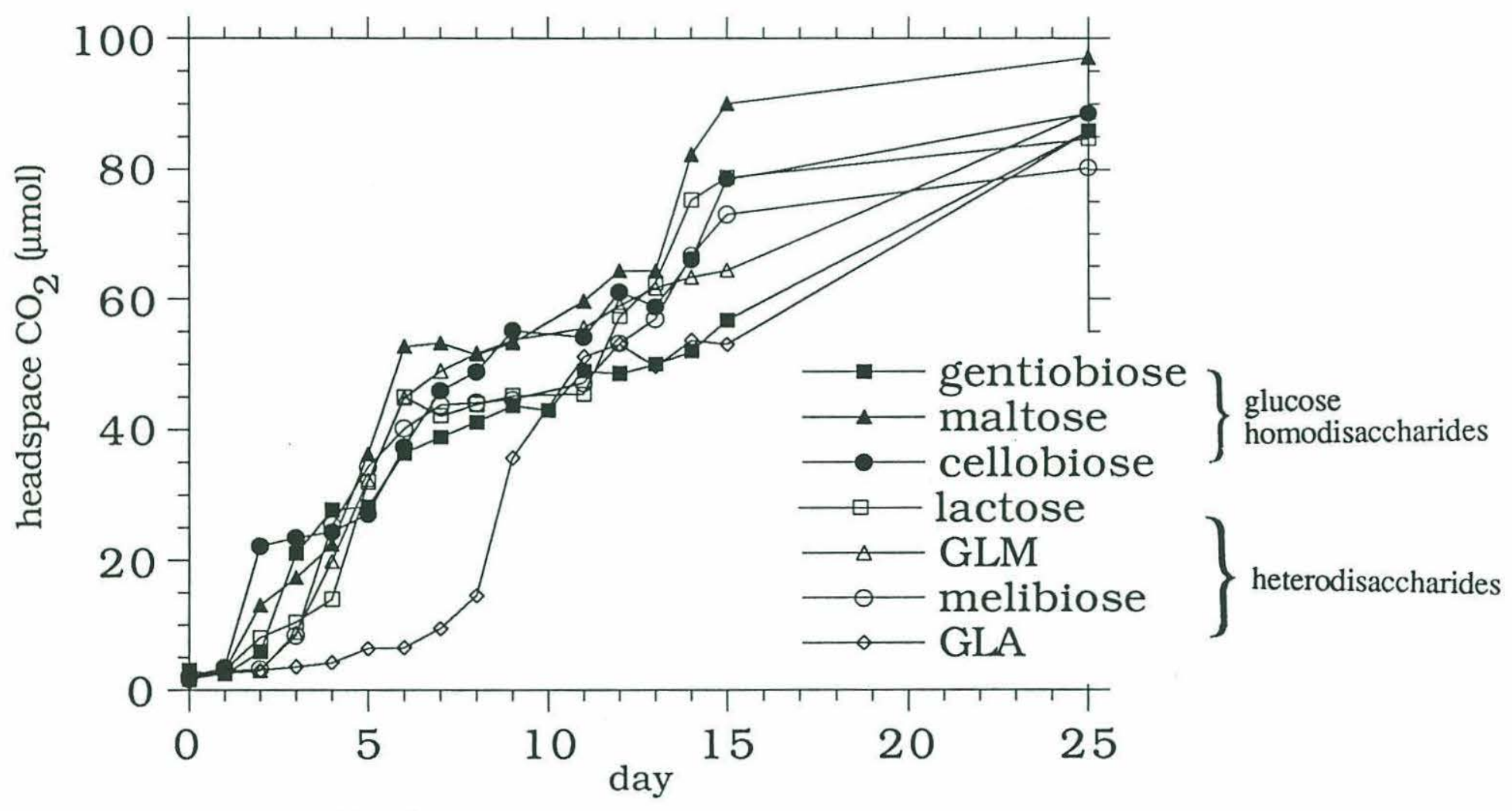

Fig. 4.7

Headspace $\mathrm{CO}_{2}$ profiles for disaccharide substrates. 
substrate (2 to 4 days) (Fig. 4.8). Again, initial $\mathrm{CO}_{2}$ levels in the headspace were closely tied to substrate degradation. The lag in degradation of substrate was mirrored by a lag in headspace $\mathrm{CO}_{2}$ (Fig. 4.7), which increased very slowly for the initial 8 days, increased abruptly between days 8 and 11, and was similar to that of other cultures between days 12 and 25.

Recovery of carbon as $\mathrm{CO}_{2}$ at the end of the culture series was comparable for GLA and maltose (Fig. 4.9). Because total $\mathrm{CO}_{2}$ was measured only three times (days 6,13 , and 15) for this series of cultures, total $\mathrm{CO}_{2}$ levels on day 25 were calculated in two ways. The solid symbols for the day 25 point in Fig. 4.9 represent the sum of $\mathrm{CO}_{2}$ in the medium measured on day 15 , and the headspace $\mathrm{CO}_{2}$ level measured on day 25 . Since an increase in headspace $\mathrm{CO}_{2}$ was measured in both cases, $\mathrm{CO}_{2}$ in medium must also have increased. The open symbols for the day 25 timepoint represent the sum of the measured headspace $\mathrm{CO}_{2}$ level plus an estimate of $\mathrm{CO}_{2}$ in medium. The estimate was made by determining the ratio of $\mathrm{CO}_{2}$ in medium to $\mathrm{CO}_{2}$ in the headspace on day 15 . The quantity of $\mathrm{CO}_{2}$ measured in the headspace on day 25 was multiplied by this ratio to arrive at an estimate of $\mathrm{CO}_{2}$ in medium.

Particularly for GLA, this calculation most likely significantly underestimates actual $\mathrm{CO}_{2}$ levels in the medium, because large quantities of organic acids were still present in the medium on day 15 , as indicated by $\mathrm{pH}$. Figure 4.10 shows headspace $\mathrm{CO}_{2}$ and medium $\mathrm{pH}$ levels for both maltose and GLA. As previously described, $\mathrm{pH}$ drops initially with production of $\mathrm{CO}_{2}$ from substrate carbon, then increases with production of $\mathrm{CO}_{2}$ from secondary metabolites. A comparison of Figs. 4.9 and 4.10 shows that the (measured) total $\mathrm{CO}_{2}$ levels of the maltose culture increased from $40 \%$ to $63 \%$ of the theoretical maximum between days 13 and 15 (Fig. 4.9), when headspace $\mathrm{CO}_{2}$ also increased and medium $\mathrm{pH}$ returned to initial values (Fig. 4.10). For GLA, however, measured total $\mathrm{CO}_{2}$ was virtually constant between days 13 and 15 , and $\mathrm{pH}$ level did not return to initial values until some time between 15 and 25 days. A lower $\mathrm{pH}$ level, driven by the presence of organic acids, would keep the ratio of medium/headspace $\mathrm{CO}_{2}$ low, and therefore the medium/headspace ratio on day 15 is almost certainly lower than the ratio on day 25 . For maltose, for example, the medium/headspace $\mathrm{CO}_{2}$ ratio was 2.9 on day 13 , and had increased to 3.4 by day 15. For GLA, the ratio was 2.6 on day 15 . Using a ratio of 3.4 for GLA on day 25, for example, would have yielded a total $\mathrm{CO}_{2}$ level of $65.9 \%$ of the theoretical maximum. 


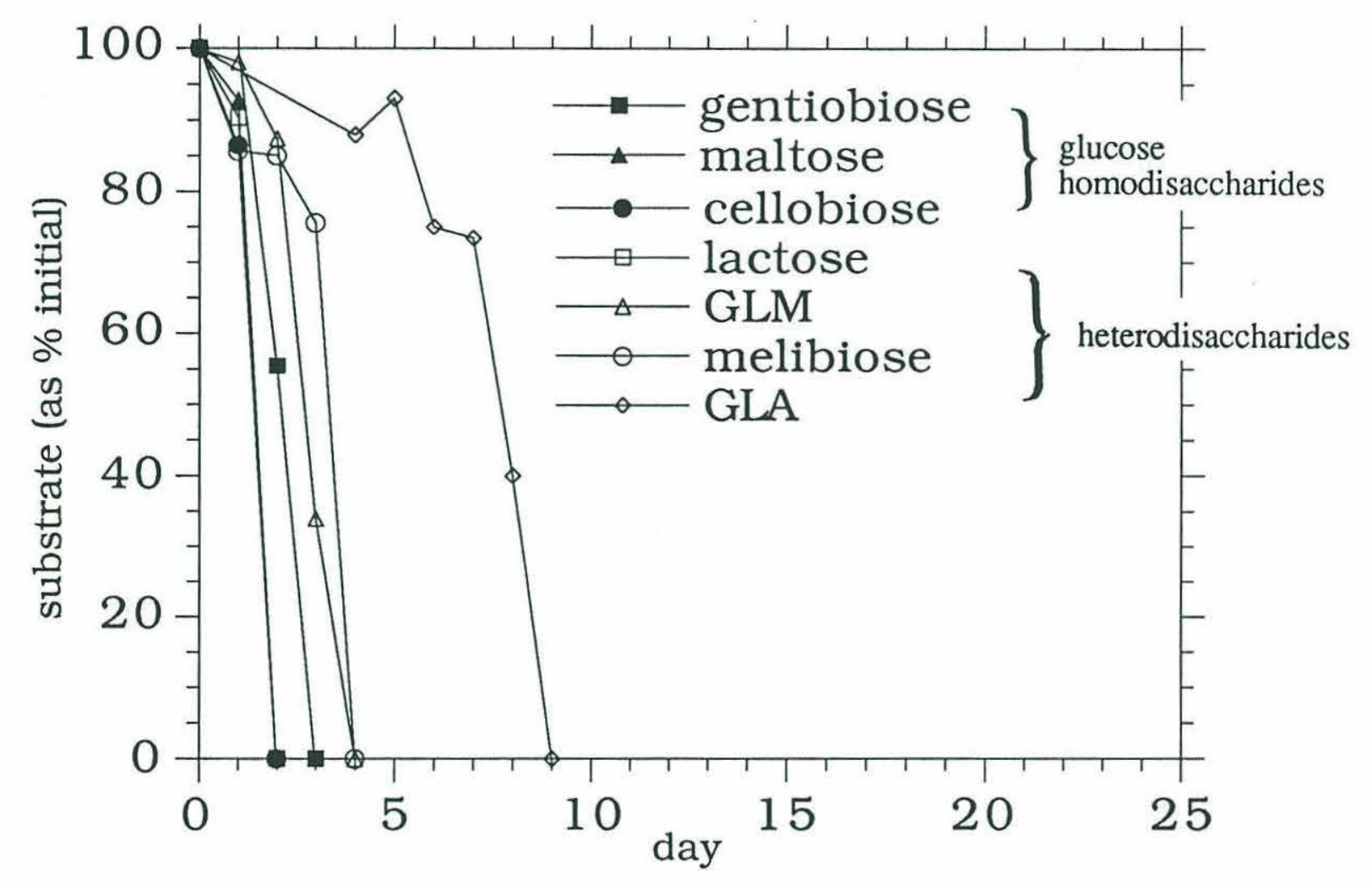

Fig. 4.8

Substrate degradation profiles for disaccharide substrates. 


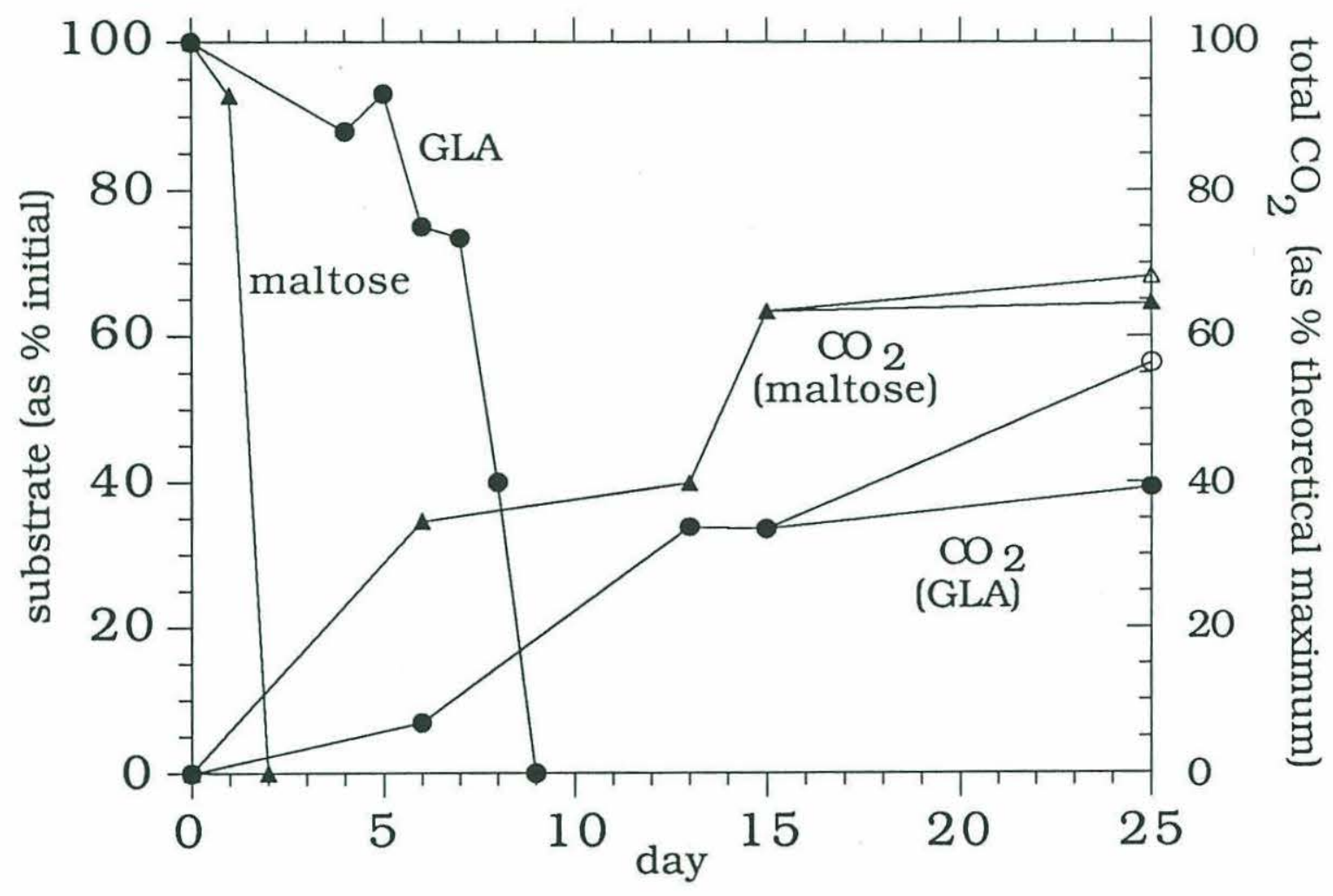

Fig. 4.9

Degradation of maltose and GLA, and evolution of total $\mathrm{CO}_{2}$. 


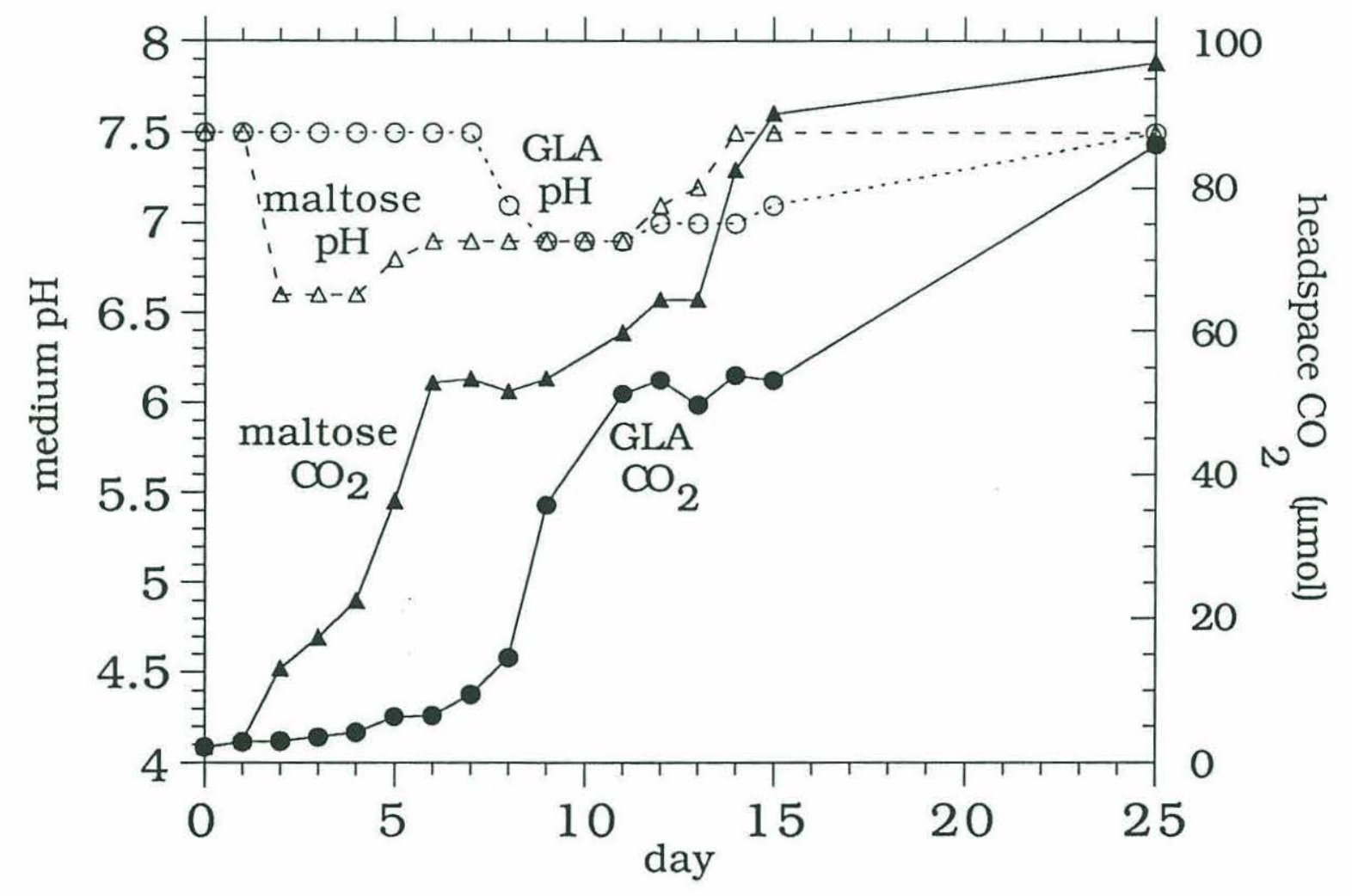

Fig. 4.10

$\mathrm{pH}$ and headspace $\mathrm{CO}_{2}$ for maltose and GLA cultures. 


\section{Degradation of GLA}

The slow degradation of GLA could have a number of causes, including the need for enzyme induction, the specific linkage or monomers of the substrate, or initial numbers of GLA-degrading bacteria. Most of these possibilities can be eliminated, however, and several lines of evidence suggest that bacterial numbers account for the lag in GLA degradation. The time lag in GLA degradation is probably not due to enzyme induction, since induction of other carbohydrate-degrading enzymes such as $\beta$-galactosidase-used in lactose degradation—occurs on the order of 30 minutes (Gottschalk, 1986). The composition of GLA is not unusual, since its component monomers, galactose and arabinose, are two of the eight neutral monosaccharides most commonly found in marine environments (Cowie and Hedges, 1984; Hamilton and Hedges, 1988). Arabinan and galactan polymers are known to occur naturally (Aspinall, 1983). Neither linkage position nor anomeric linkage are unusual, since $\beta(1,3)$ linkages are found in a range of marine carbohydrates, including chrysolaminarin, a storage product of prymnesiophytes (Lee, 1980 ). In addition, $\beta(1,3)$ hydrolase activity has been demonstrated in a number of diatoms (Myklestad et al., 1982) and has been detected in beach sands collected from beneath decomposing macrophytes (Wainwright, 1981). It is therefore unlikely that GLA is intrinsically difficult to degrade because of its component monomers or linkage.

To determine whether bacterial numbers significantly affected GLA degradation, a GLA culture was grown until day 10 , when the headspace $\mathrm{CO}_{2}$ increased sharply, and 0.5 $\mathrm{mL}$ medium was then used to inoculate a second vial. As shown in Fig. 4.11, the lag in $\mathrm{CO}_{2}$ production in the transfer culture was half of that in the original culture. These results suggest that the rate of degradation of GLA is related to the number of GLA-degrading bacteria present in the initial inoculum. The ability to degrade GLA is probably not widespread among bacteria, and a relatively uncommon bacterium is responsible for GLA degradation.

Why might only select bacteria have the ability to degrade GLA, when both the component monomers of GLA and $\beta(1,3)$ linkages are common in marine systems? The unusual feature of GLA might be its hexamer-pentamer combination: arabinose lacks a \#6 carbon, which might be a critical feature in a substrate-enzyme complex used to handle most disaccharides. GLA is sufficiently small to be ingested without extracellular hydrolysis, so the critical step might be either intracellular transport of the dimer (i.e. from the periplasmic space through the cell membrane), or actual hydrolysis of the bond within the cell. 


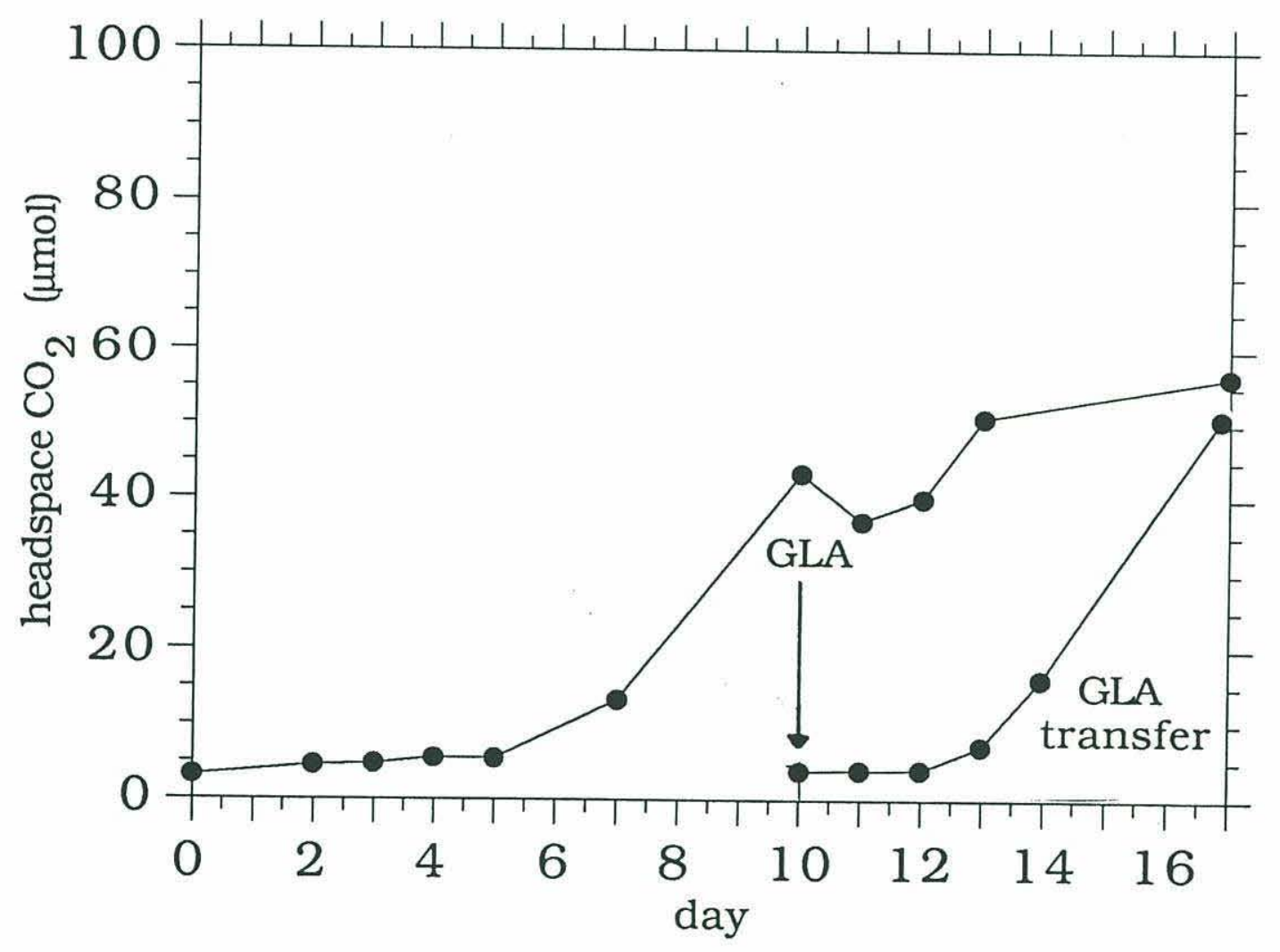

อे

Fig. 4.11

Headspace $\mathrm{CO}_{2}$ for GLA culture and transfer culture. On day $10,0.5 \mathrm{~mL}$ of the GLA culture was used to inoculate the transfer culture. Evolution of $\mathrm{CO}_{2}$ (and degradation of substrate) were much more rapid for the transfer culture than for the initial culture. 
Intracellular transport of carbohydrates has been studied extensively in a few gramnegative bacteria, and several different systems which transport carbohydrates into the cytoplasm have been identified. Group translocation systems modify a substrate-for example, by adding a phosphate group — during transport, while permease systems cotransport the unaltered substrate and $\mathrm{H}^{+}$(or in some cases $\mathrm{Na}^{+}$or $\mathrm{Li}^{+}$) (Nikaido and Saier Jr., 1992). Binding-protein permeases, which require ATP, are also common in gramnegative bacteria (Gottschalk, 1986). Glucose and other monosaccharides are often transported via group translocation systems, such as the phosphoenolpyruvatephosphotransferase system, which phosphorylates hexoses at the \#6 carbon as they are transported. Although not found in all bacteria, phosphotransferase systems are common in anaerobes. Two glucose phosphotransferase systems have been identified in the gramnegative bacterium Escherichia coli. One system is specific for glucose, while the other can transport glucose, mannose, and "other sugars with different substituents at carbon 2 of the hexose ring" (Erni, 1989). The same carbohydrate may be transported using different systems in different bacteria. For example, some bacteria transport disaccharides such as lactose using group translocation, while others transport lactose via a permease system (Gottschalk, 1986). The same bacterium may also contain a variety of transport systems which become active under different environmental conditions. In bacteria with permease systems for disaccharides, internal degradation often occurs through phosphorylytic cleavage. Cellobiose and maltose, for example, are internally hydrolyzed to glucose-1phosphate and glucose (Gottschalk, 1986).

A common feature of the transport and hydrolysis systems is that the proteins are specific for a substrate or a small range of substrates. For phosphorylation and phosphorylytic cleavage, the \#6 and \#1 carbons are crucial. In the case of GLA, the hexamer-pentamer substrate combination may be unusual enough that relatively few bacteria have the biochemical machinery necessary to transport or hydrolyze GLA. Without a \#6 carbon on one of its component monomers, GLA might not fit a standard template. If the ability to degrade this hexamer-pentamer combination is relatively rare, the long initial delay in degradation observed in the culture experiments would be a reflection of the amount of time necessary for a relatively uncommon bacterium to reach numbers sufficient to degrade all of the substrate. In the absence of information on the structure of most marine oligo- and polysaccharides, the natural occurrence of these types of structures in marine environments cannot be assessed. 


\section{Degradation of Maltooligosaccharides}

In order to determine whether there were any distinguishable differences between degradation of a size range of oligosaccharides, and in particular whether any lower molecular weight oligosaccharides were produced and released into the medum as intermediates during degradation, a series of maltooligosaccharides were used as substrates in a culture series. All substrate concentrations were adjusted to a $1 \mathrm{mM}$ dimer equivalent level: 1 mM DP2 (maltose), .667 mM DP3 (maltotriose), . 400 mM DP5 (maltopentaose), and $.286 \mathrm{mM} \mathrm{DP7}$ (maltoheptaose). The degradation of the maltooligosaccharides occurred very quickly, and the profiles of substrate disappearance and production of total $\mathrm{CO}_{2}$ were virtually identical (Fig. 4.12). HPLC analysis of the medium showed that no lower molecular-weight oligosaccharides accumulated in the medium as degradation intermediates in any of the cultures.

The uniform pattern of substrate degradation in the DP2-DP7 maltooligosaccharide cultures was somewhat surprising. In general, DP5 and DP7 oligosaccharides are too large to be directly taken up, and must be hydrolyzed outside the cell by exoenzymes before they can be utilized by bacteria. The nearly identical pattern of substrate uptake, and the lack of observable lower molecular weight oligosaccharides in the maltopentaose (DP5) and maltoheptaose (DP7) cultures, could have two possible explanations. Either extracellular hydrolysis and uptake are very closely coupled, so that intermediates were taken up too quickly to accumulate to detectable levels, or some of the bacteria in the cultures have special uptake channels for maltose, as has been observed for E. coli, another common gram-negative bacterium.

As discussed in Chapter 1, bacteria take up hydrophilic substrates such as carbohydrates through trimeric proteins which form water-filled pores (porins) which span the bacterial outer membrane. These porins typically have an uptake limit of 600 daltons (Weiss et al., 1991), which is approximately the equivalent of a trisaccharide. Maltoporin is an inducible porin (i.e., expressed only in the presence of maltooligosaccharides) which has been extensively studied in E. coli. These studies (Benz, 1988, and references therein) have shown that maltooligosaccharides up to maltoheptaose can be directly taken up through a maltoporin. If maltoporin is also found in some species marine bacteria, then the entire maltooligosaccharide series could be taken up directly without extracellular hydrolysis, so that substrate degradation and evolution of $\mathrm{CO}_{2}$ would be the same for each member of the maltooligosaccharide series. The structure and substrate specificity of porins has been extensively studied, but the porins have come from a restricted range of bacteria. A search of the literature revealed that references to maltoporin are limited to $E$. coli. The 


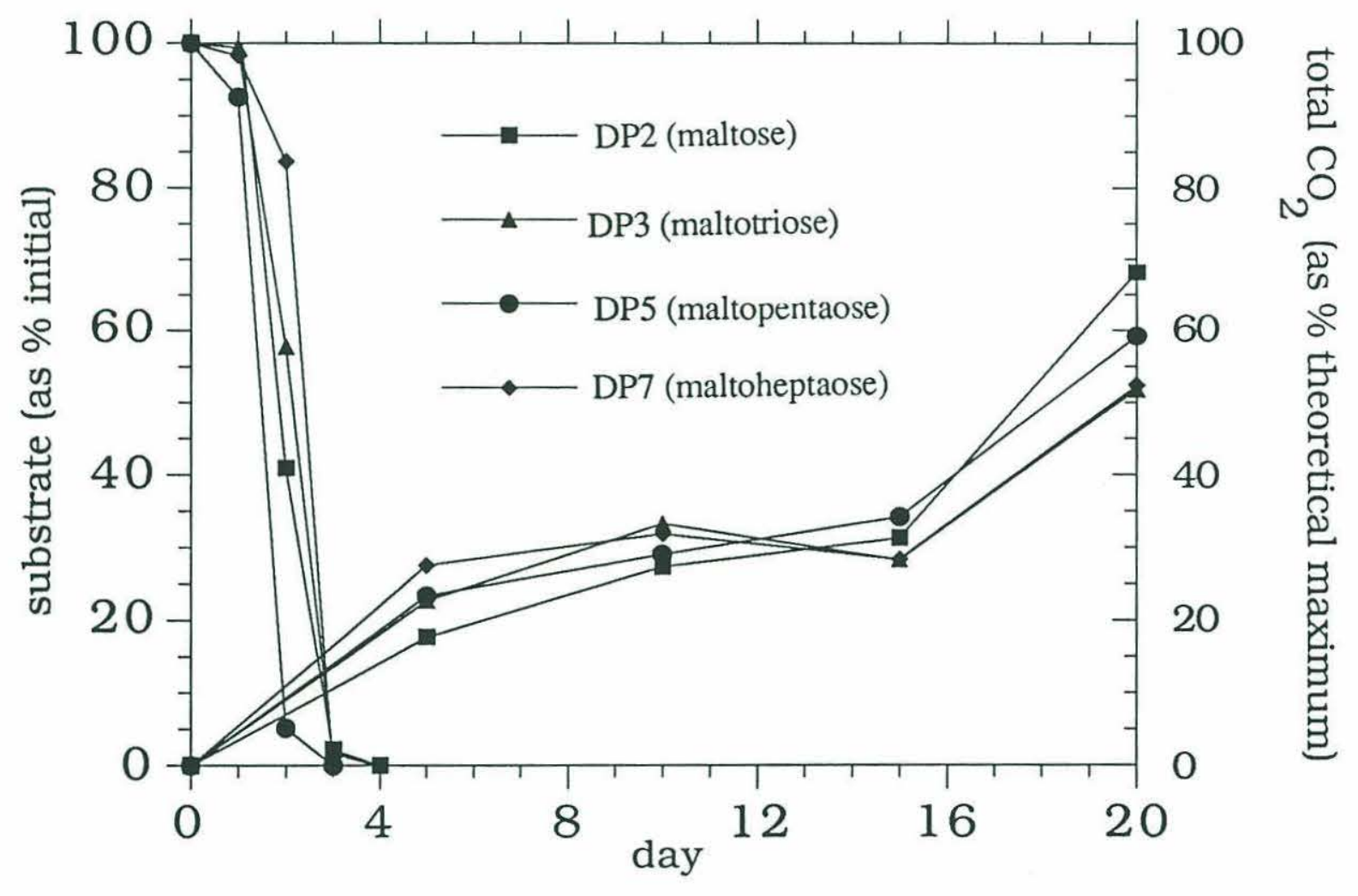

Fig. 4.12

Substrate degradation and evolution of total $\mathrm{CO}_{2}$ for maltooligosaccharide series. 
only studies which have been carried out on marine bacteria focused on structural determinations of the general diffusion porins of the marine phototrophs Rhodobacter capsulatus (Weiss et al., 1990) and Rhodobacter sphaeroides (Benz, 1988; Nikaido and Saier Jr., 1992, and references therein).

A study of the degradation of mixtures of DP2-DP6 cellooligosaccharides by pure cultures of rumen bacteria showed that while extracellular hydrolysis and uptake can be closely coupled, changes in relative distribution of the cellooligosaccharides can be detected. As concentrations of cellohexaose and cellopentaose in the culture medium decreased, concentrations of lower molecular weight cellooligosaccharides increased. Although complete degradation of the cellooligosaccharides occurred on a timescale of hours, the changes in cellooligosaccharide composition were clearly resolved using HPLC analysis of the medium (Russell, 1985). Pure cultures of starch-degrading rumen bacteria also produced a range of oligosaccharides during the initial steps of degradation. Cocultures of these bacteria produced detectable levels of maltooligosaccharides from starch in most, but not all, cases. Uptake of maltooligosaccharides in one co-culture was swift enough that starch degradation products were not detected, even though the polysaccharidedegrading member of the co-culture had been shown to produce a range of maltooligosaccharide intermediates from starch when grown in pure culture (Cotta, 1992).

Either scenario-maltoporin, or closely coupled hydrolysis and uptake-is possible for the maltooligosaccharide cultures. In all cases, no accumulation of smaller oligosaccharides in the medium was observed, even for time points at which the original substrate was partly consumed. Better resolution might be achieved by taking more frequent and larger samples of the medium during the initial 72 hours of the culture. One way to distinguish between the two possibilities would be to try a similar series of culture experiments using cello- or xylo-oligosaccharides, which would have to be hydrolyzed extracellularly. If no lower molecular weight oligomers were observed in these cases, uptake must be closely coupled to hydrolysis. If lower molecular weight oligosaccharides did accumulate, however, it would leave an unresolved dilemma, since the relative uptake affinities for maltooligosaccharides may differ from those for xylo- or cellooligosaccharides.

\section{Conclusions}

Although carbohydrate dimers are well within the size range which can be ingested without extracellular hydrolysis (Gottschalk, 1986; Priest, 1992), the di- and oligosaccharide experiments indicate that structural differences can be important in 
determining degradation rate even at a small size level. The maltooligosaccharide experiments illustrate that in certain cases—such as GLA and maltoheptaose-small structural features may be more important than molecular weight or size in determining relative rate of degradation. Individual species of bacteria are known to preferentially ingest certain mono- and disaccharides (Bernier and Stutzenberger, 1987; Hespell et al., 1987); these experiments demonstrate that even mixed bacterial cultures, comprised of a variety of bacterial types, distinguish between small substrates. The slight initial delay in degradation of GLM and melibiose, as well as the much longer delay in GLA degradation, are probably a reflection of the time necessary for bacteria with the proper enzyme systems to double to sufficient numbers for substrate degradation to be detected. Enzymatic capability to degrade glucose homodimers, regardless of linkage type or position, might be more widely distributed than the ability to degrade heterodimers.

Although the oligosaccharide degradation experiments took place on time scales of weeks, not years, evidence that small variations in structure can lead to measurable differences in rate of substrate degradation even for simple, highly soluble carbohydrates has wider implications for the degradation of polysaccharides in marine sediments. These experimental results suggest that varying rates of organic polymer degradation in anoxic sediments may be determined in part by the sensitivity of bacterial enzymatic and transport systems to structural features. Some types of marine carbohydrates may be very difficult to degrade, primarily because of their specific chemical structure. Factors such as packaging or particle interaction might further slow the degradation of nominally labile polysaccharides. In areas with high rates of productivity and sedimentation, greater relative resistance to degradation may result in some of the original polysaccharide being buried to a depth below the zone of most active remineralization. 


\section{References}

Aspinall, G. O. (1983). Classification of polysaccharides. The Polysaccharides. New York, Academic Press. 1-9.

Benz, R. (1988). Structure and function of porins from gram-negative bacteria. Ann. Rev. Microbiol. 1988 : 359-393.

Bernier, R. and F. Stutzenberger (1987). Preferential utilization of cellobiose by Thermomonospora curvata. Appl. Environ. Microbiol. 53(8): 1743-1747.

Biddanda, B. A. (1988). Microbial aggregation and degradation of phytoplankton-derived detritus in seawater. II. Microbial metabolism. Mar. Ecol.Prog. Ser. 42: 89-95.

Burney, C. M. (1986). Bacterial utilization of total in situ dissolved carbohydrates in offshore waters. Linmol. Oceanogr. 31(2): 427-431.

Clark, D. P. (1989). The fermentation pathways of Escherichia coli. FEMS Micro. Rev. 63: 223-234.

Cole, J. J., G. E. Likens and D. L. Strayer (1982). Photosynthetically produced dissolved organic carbon: An important carbon source for planktonic bacteria. Limnol. Oceanogr. 27(6): 1080-1090.

Cotta, M. A. (1992). Interaction of ruminal bacteria in the production and utilization of maltooligosaccharides from starch. Appl. Env. Microbiol. 58(1): 48-54.

Cowie, G. L. and J. I. Hedges (1984). Carbohydrates sources in a coastal marine environment. Geochim. Cosmochim. Acta 48: 2075-2087.

Erni, B. (1989). Glucose transport in Escherichia coli. FEMS Microb. Rev. 63: 13-24.

Gocke, K., R. Dawson and G. Liebezeit (1981). Availability of dissolved free glucose to heterotrophic microorganisms. Mar. Biol. 62: 209-216.

Gottschalk, G. (1986). Bacterial Metabolism. Berlin, Springer-Verlag.

Hama, T. and N. Handa (1987). Pattern of organic matter production by natural phytoplankton population in a eutrophic lake 2. Extracellular products. Arch. Hydrobiol. 109(2): 227-243.

Hamilton, S. E. and J. I. Hedges (1988). The comparative chemistries of lignins and carbohydrates in an anoxic fjord. Geochim. Cosmochim. Acta 52: 129-152.

Hespell, R. B., R. Wolf and R. J. Bothast (1987). Fermentation of xylans by Butyrivibrio fibrisolvens and other ruminal bacteria. Appl. Environ. Microbiol. 53(12): 2849-2853.

Hungate, R. E. (1969). A roll tube method for cultivation of strict anaerobes. Methods in Microbiology. New York, Academic Press.

Iturriaga, R. and A. Zsolnay (1983). Heterotrophic uptake and transformation of phytoplankton extracellular products. Bot. Mar. 26: 375-381. 
Khailov, K. M. (1968). Extracellular microbial hydrolysis of polysaccharides dissolved in seawater. Mikrobiologiya 37(3): 518-522.

Kuivila, K. M., J. W. Murray and A. H. Devol (1990). Methane production in the sulfatedepleted sediments of two marine basins. Geochim. Cosmochim. Acta 54: 403-411.

Lee, C. (1992). Controls on organic carbon preservation: the use of stratified water bodies to compare intrinsic rates of decomposition in oxic and anoxic systems. Geochim. Cosmochim. Acta 56: 3323-3335.

Lee, R. E. (1980). Phycology. Cambridge, Cambridge University Press.

Meyer-Reil, L. A. (1978). Uptake of glucose by bacteria in sediments. Mar. Biol. 44: 293298.

Myklestad, S., R. Djurhuus and A. Mohus (1982). Demonstration of exo-( $\beta-1,3)$-Dglucanase activity in some planktonic diatoms. J. exp. mar. Biol. Ecol. 56: 205-211.

Newell, R. C., M. I. Lucas and E. A. S. Linley (1981). Rate of degradation and efficiency of conversion of phytoplankton debris by marine microorganisms. Mar. Ecol. Prog. Ser. 6: 123-136.

Nikaido, H. and M. H. Saier Jr. (1992). Transport proteins in bacteria: common themes in their design. Science 258: 936-942.

Oremland, R. S. and S. Polcin (1982). Methanogenesis and sulfate reduction: competitive and noncompetitive substrates in estuarine sediments. Appl. Environ. Microb. 44(6): 1270-1276.

Priest, F. G. (1992). Synthesis and secretion of extracellular enzymes in bacteria. Microbial Degradation of Natural Products. Weinham, VCH. 1-26.

Russell, J. B. (1985). Fermentation of cellodextrins by cellulolytic and noncellulolytic rumen bacteria. Appl. Environ. Microbiol. 49(3): 572-576.

Sakugawa, H. and N. Handa (1985). Chemical studies on dissolved carbohydrates in the water samples collected from the North Pacific and Bering Sea. Oceanol. Acta 8(2): 185196.

Sundh, I. (1989). Characterization of phytoplankton extracellular products (PDOC) and their subsequent uptake by heterotrophic organisms in a mesotrophic forest lake. $J$. Plankton Res. 11(3): 463-486.

Takahashi, M. and S. Ichimura (1971). Glucose uptake in ocean profiles with special reference to temperature. Mar. Biol. 11: 206-213.

Vaccaro, R. F. and H. W. Jannasch (1966). Studies on heterotrophic activity in seawater based on glucose assimilation. Limnol. Oceanogr. 11: 596-607.

Wainwright, M. (1981). Assay and properties of alginate lyase and 1,3- $\beta$-glucanase in intertidal sands. Plant and Soil 59(1): 83-89. 
Weimer, P. J. and J. G. Zeikus (1977). Fermentation of cellulose and cellobiose by Clostridium thermocellum in the absence and presence of Methanobacterium thermoautotrophicum. Appl. Environ. Microbiol. 33(2): 289-297.

Weiss, M. S., U. Abele, J. Weckesser, W. Welte, E. Schiltz and G. E. Schulz (1991). Molecular architecture and electrostatic properties of a bacterial porin. Science 254: 1627 1630.

Weiss, M. S., T. Wacker, J. Weckesser, W. Welte and G. E. Schulz (1990). The threedimensional structure of porin from Rhodobacter capsulatus at $3 \AA$ resolution. FEBS Lett. 267(2): 268-272.

Widdel, F. (1988). Microbiology and ecology of sulfate- and sulfur-reducing bacteria. Biology of Anaerobic Microorganisms. New York, Wiley. 469-585.

Winfrey, M. R., D. G. Marty, A. J. M. Bianchi and D. M. Ward (1981). Vertical distribution of sulfate reduction, methane production, and bacteria in marine sediments. Geomicrob. J. 2(4): 341-362.

Wood, W. A. (1961). Fermentation of Carbohydrates and Related Compounds. The Bacteria. New York, Academic Press. 59-149. 


\section{Chapter Five \\ Effect of Chemical Structure on Carbohydrate Degradation by Anaerobic Marine Bacteria: Degradation of Polysaccharides}

\section{Introduction}

The previous series of culture experiments demonstrated that the culture system produced robust, reproducible results. Small differences in degradation lag between homo-and hetero-disaccharides, as well as the long degradation lag in the case of the GLA substrate, suggest that bacteria differentiate between closely related substrates even when extracellular hydrolysis is not required. In terms of the model shown in Fig. 1.1, the previous cultures involved transitions from the pool of 'dissolved monomers' (which here are defined as substrates which bacteria can ingest without extracellular hydrolysis) to methane and $\mathrm{CO}_{2}$.

Most organic matter is synthesized as macromolecules, which bacteria must hydrolyze with exoenzymes before they can transport and convert them to cellular carbon and $\mathrm{CO}_{2}$. The enzymatic hydrolysis of macromolecules is often assumed to be the slow step in organic matter degradation (Meyer-Reil, 1987; Hoppe, 1991, for example; MeyerReil, 1991), but this assumption has not been rigorously tested. The experiments in Chapter 4 demonstrated that small variations in substrate structure can greatly restrict the range of bacteria which are capable of uptake and intracellular degradation of a small carbohydrate. By comparing the results of the di- and oligosaccharide degradation experiments with the degradation of structurally-related polysaccharides, effects which are solely attributable to molecular size can be determined. The transitions represented by $\mathrm{F}$ and E in Fig. 1.1 (dissolved polymers $\longrightarrow$ dissolved monomers, and dissolved monomers $\longrightarrow$ methane $+\mathrm{CO}_{2}$, respectively) can thereby be differentiated.

Although little work has been done on the bacterial degradation of macromolecules such as carbohydrates in marine systems, extensive references are available in the nonmarine literature. The best reference point in any study of anaerobic carbohydrate degradation are studies of the natural consortia of ruminants. In cattle and other ruminants, a complex population of strictly anaerobic bacteria cooperatively degrade plant polysaccharides. Studies of rumen bacteria are also the basis of much contemporary work on anaerobes, since the techniques commonly used to culture anaerobic bacteria ("Hungate techniques") were originally developed to work with rumen bacteria (Hungate, 1969).

Extensive studies of bacterial carbohydrate degradation have been carried out with pure cultures, co-cultures, and consortia of rumen bacteria over the past several decades (Hungate, 1966; Dehorty, 1967; Weimer and Zeikus, 1977; Miura et al., 1983; Hespell et 
al., 1987; Cotta, 1992; Malburg et al., 1992, for example). These studies have elucidated the biochemical basis of carbohydrate degradation by anaerobic bacteria, and have provided a fundamental understanding of anaerobic metabolism. Rumen bacteria have a wide range of enzymatic and metabolic capabilites, and are nutritionally interdependent. Plant polysaccharides are degraded by exoenzymes excreted by cellulolytic* bacteria. As discussed in Chapter 1, these bacteria ferment oligosaccharides and excrete low molecular weight substrates such as alcohols and fatty acids, which in turn are utilized by other bacteria. The non-cellulolytic bacteria may, for example, excrete vitamins required by some of the cellulolytic bacteria, or may consume $\mathrm{H}_{2}$ produced during fermentation. In this manner, bacteria which can utilize only a limited range of low molecular weight substrates cooperate with metabolically more versatile bacteria.

Studies of the exoenzymes produced by rumen bacteria have also yielded detailed information about the mechanisms and specificities of enzymatic carbohydrate degradation (Williams and Withers, 1982; Robson and Chambliss, 1989). Further information on carbohydrate-degrading enzymes has been provided by studies of enzymes from thermophilic and extreme thermophilic bacteria, which are of interest due to their applications in biotechnology (paper pulping, starch saccharification) (Hudson et al., 1990; Klingeberg et al., 1990; Koch and Antranikian, 1990). The enzymes of several marine thermophiles and extreme thermophiles isolated from hydrothermal vents have also been studied in this context (Brown et al., 1990; Koch et al., 1990).

The bacterial (as differentiated from the isolated enzymatic) conversion of polysaccharides to oligosaccharides, and the subsequent degradation of the oligosaccharides, however, has been followed in detail in only a few studies of pure- and co-cultures of rumen bacteria (Russell, 1985; Cotta, 1992), and has not been studied at all with either pure cultures or consortia of aquatic bacteria.

As discussed in Chapter 1, although studies of rumen bacteria are an invaluable source of information on the degradation of carbohydrates by anaerobes, rumen consortia differ from marine anaerobic consortia in several important aspects. The rumen is a natural bioreactor, in which the feedstock is chemically consistent (principally plant polysaccharides) and is fed in and washed out at a constant rate. The organic matter reaching rumen anaerobes is always fresh, and the system is thermally very stable. In addition, the terminal members of rumen consortia are methanogenic bacteria, whereas high

\footnotetext{
"The term "cellulolytic" is used here to refer to all polysaccharide-degrading rumen bacteria. Only a fraction of these organisms actually are cellulolytic; the majority hydrolyze starch and hemicellulose contined in plant polysaccharides.
} 
levels of sulfate in marine systems results in the predominance of sulfate reducers as terminal members of marine bacterial consortia.

The anaerobic degradation of macromolecular organic matter in marine systems therefore requires further investigation. Most models of carbon cycling in the marine environment assume the conversion of high molecular weight organic matter to lower molecular weight substrates (Burdige and Martens, 1988; Henrichs, 1992). The specifics of these conversion processes, though, have not been studied. As discussed in Chapter 1, "G-model"-type studies indicate that there is a range of reactivity classes of organic matter in marine sediments, although the factors determining the range of reactivity are unknown. An important goal of the culture studies was to investigate the bacterial degradation of polysaccharides in order to determine the effects of gross size and chemical linkage on degradation rates and patterns. This chapter presents the results of a series of experiments in which replicate cultures of anaerobic marine bacteria were enriched on polysaccharides. Sampling time points were very closely spaced in order to follow degradation processes in detail. As in the culture experiments discussed in Chapter 4, the disappearance of substrate and the production of gaseous end products was monitored. In addition, gel permeation chromatography (GPC) and nuclear magnetic resonance spectroscopy (NMR) were used to follow the extracellular conversion of high-molecular weight polysaccharides to lower molecular weight poly- and oligosaccharides which were subsequently also degraded.

\section{Methods}

Substrates

The substrates were laminarin (a $\beta(1,3)$ linked glucose polysaccharide, MW 5-6000 daltons), pullulan ( $\alpha(1,6)$-linked maltotriose units, MW 200,000 daltons), and F2 and F3, the branched glucose polysaccharide and teichoic acid-type polymer isolated from Synechococcus WH7335 (Chapter 3). NMR spectra of all substrates were presented in Chapters 2 (laminarin and pullulan, Figs. 2.17 and 2.18) and 3 (F2 and F3, Figs. 3.8 and 3.11). The pullulan was used as received from U.S. Biochemicals, and F2 and F3 were used as the isolates described in Chapter 3. Tests with laminarin showed that as received from U.S. Biochemicals, the laminarin contained insoluble material; in addition, after a filtered laminarin solution sat at room temperature for several days, a soft insoluble gel precipitated. The laminarin was therefore dissolved, filtered through a $0.2 \mu$ filter, and lyophilized before weighing. The starting material was a dense medium-brown powder, which became white and fluffy after lyophilization. The laminarin solution was filtered 
again upon addition to the cultures. Laminarin prepared in this manner was more soluble than the starting material, and did not precipitate from solution.

Substrate concentrations of laminarin and pullulan were equivalent (on a carbon basis) to the $1.0 \mathrm{mM}$ level used for the disaccharide cultures in Chapter 4. Final substrate concentrations (after addition of inoculum) were $336 \mu \mathrm{g} / \mathrm{mL}$ for laminarin and pullulan (equivalent to $67 \mu \mathrm{M}$ and $1.7 \mu \mathrm{M}$, respectively). The fraction of $\mathrm{F} 2$ with a nominal molecular weight $>5000$ daltons upon re-chromatography (see Chapter 3 ) was used in the culture. Approximately $380 \mu \mathrm{g}$ of F2 were added to a culture vial. Monosaccharide analysis (see Chapter 3) showed that the carbohydrate concentration of F2 was $146 \mu \mathrm{g} / \mathrm{mg}$. The 11 $\mathrm{mL}$ culture therefore had a carbohydrate concentration of $5.1 \mu \mathrm{g} / \mathrm{mL}$.

Fifty mg of substrate were used in the F3 culture. The amount of carbohydrate added can only be approximated. While $\mathrm{CHN}$ analysis of $\mathrm{F} 3$ showed that the weight ratio of $\mathrm{F} 3$ was $3.98 \%: 0.67 \%: 0.74 \%$, monosaccharide analyses (see Chapter 3) suggested that F3 was approximately $50 \%$ carbohydrate. Both measurements of $\mathrm{F} 3$ may be accurate; after lyophilization, F3 was a waxy or crusty tan-white material which may have been heterogeneous in composition. As discussed in Chapter 3, most of F3 was used for the culture series and carbohydrate analysis; only a small residual, which may have been enriched in salts, was available for CHN analysis. The high-field material observed in the ${ }^{1} \mathrm{H}$ NMR spectrum (Fig. 3.5) indicated that some of the nitrogen could come from proteins, but the ${ }^{13} \mathrm{C}$ NMR spectra (Figs. $3.14,3.15$ ) showed that all major and minor carbons could be unambiguously assigned to the teichoic acid-type polysaccharide. If protein had been a major component of the $\mathrm{F} 3$ extract, corresponding carbon resonances should have been observed. The charged teichoic acid-type polysaccharide most likely contains salt as counter ions; ammonium salts, for example, could be a source of some of the nitrogen.

The structure proposed for $\mathrm{F} 3$ has a C:H:O:P ratio of 34.2\%: 5.3\%: 51.3\%: 9.2\%. As an approximation, therefore, the $\mathrm{F} 3$ sample for $\mathrm{CHN}$ analysis may be $10 \%$ carbohydrate (which would leave a residual of $0.56 \% \mathrm{C}: 0.14 \% \mathrm{H}: 0.74 \% \mathrm{~N}$ for the noncarbohydrate material; some of the nitrogen may be in the form of $\mathrm{N}$-salts). At $10 \%$ carbohydrate composition, the approximate concentration of $\mathrm{F} 3$ in the culture would be 93 $\mu \mathrm{g} / \mathrm{mL}$; with a carbohydrate concentration of $50 \%$, the concentration of $\mathrm{F} 3$ would be $\sim 470$ $\mu \mathrm{g} / \mathrm{mL}$. 


\section{Culture methods}

Preparation of medium, substrate, and inoculum were as described in Chapter 4. The only difference was that the sediment used for the inoculum was freshly collected from Salt Pond in October 1992 and stored at $4^{\circ} \mathrm{C}$ for 4 months prior to use. In addition, because polysaccharides are less soluble than disaccharides, the substrates were dissolved in larger volumes of $\mathrm{Q}-\mathrm{H}_{2} \mathrm{O}$, and $4.0 \mathrm{~mL}$ of substrate solution was added to each $50 \mathrm{~mL}$ culture.

Cultures with laminarin and pullulan were run in triplicate, while due to limited substrate quantities, only single vials of F2 and F3 were prepared. Blanks included vials with substrate and no inoculum (pullulan and laminarin; "B" vials), inoculated vials which were autoclaved prior to addition of substrate (pullulan, laminarin, and F3 "IAS" vials), and a vial which was inoculated without addition of substrate ("I" vial).

\section{Culture Sampling}

Samples for headspace gas analysis, total $\mathrm{CO}_{2}, \mathrm{pH}$, and substrate concentration were collected as described in Chapter 4. Samples of culture medium were collected at intervals of 4-8 hours over the first 70 hours of the culture experiment, while headspace gases were measured every 24 hours for the first three days, and then on the 5 th, 8 th, 12 th, 15 th, and 26 th days after inoculation. Total $\mathrm{CO}_{2}$ was measured only on the 15 th and 26th days after inoculation. Only four medium samples were collected for F2. At 0 and at 69 hours, 1.5 $\mathrm{mL}$ of medium was removed from the culture; $1 \mathrm{~mL}$ was removed for total $\mathrm{CO}_{2}$ determination at 15 and 26 days. Removal of additional medium from the F2 culture at intermediate timepoints would have excessively drained the $11 \mathrm{~mL}$ culture.

\section{Sample Analysis}

Headspace gases were analyzed by gas chromatography, as described in Chapter 4 . The sensitivity of the thermal conductivity detector had deteriorated over a two-year interval, so that detection limits were 360,260 , and $4 \mathrm{ppm}$ by volume for $\mathrm{CO}_{2}, \mathrm{CH}_{4}$, and $\mathrm{H}_{2}$, respectively.

Samples were analyzed for carbohydrates by using a combination of gel permeation chromatography (GPC) and NMR spectroscopy. The objectives in using GPC were both to remove salts from the higher molecular weight fractions in order to accurately quantify remaining polysaccharides by refractive index detection, and to separate the remaining substrate into discrete molecular weight classes. At each time point, $1.0 \mathrm{~mL}$ medium was 
removed from each culture vial, and sterile filtered through a $0.2 \mu \mathrm{m}$ syringe filter into a clean vial. A few samples were analyzed immediately, and the rest were stored frozen at $40^{\circ} \mathrm{C}$ until analysis. Two different gel permeation columns were used to separate carbohydrates. A Sephadex G-25 column was used to analyze all of the laminarin samples, the two F2 samples, and to monitor a few pullulan samples during the first 70 hours of the cultures, while a complete series of pullulan samples was analyzed on a G-50 column. The Sephadex G-25 column (100-300 $\mu$ m mesh, nominal exclusion on a dextran basis: 5000 daltons) was $1.5 \times 33.8 \mathrm{~cm}$., with a void volume of $27.3 \mathrm{~mL}$ (corresponding to $24.8 \mathrm{~min}$ ) as calibrated with pullulan, and a total volume of $59.8 \mathrm{~mL}$. The Sephadex G-50 column (nominal exclusion: 10,000 daltons, $100-300 \mu \mathrm{m}$ mesh) was used to fractionate the pullulan samples. The G-50 column was $1.5 \times 40.6 \mathrm{~cm}$, with a void volume of $25.8 \mathrm{~mL}$ (as calibrated with pullulan, MW 200,000 daltons) and a total volume of $71.7 \mathrm{~mL}$. The mobile phase in both cases was $\mathrm{Q}-\mathrm{H}_{2} \mathrm{O}$, pumped at $1.1 \mathrm{~mL} / \mathrm{min}$ with a peristaltic pump (Pharmacia P-1). Carbohydrates were detected with a refractive index detector (Showdex RI-71, detection limit ca. $5 \mu \mathrm{g}$ carbohydrate), and data were acquired with a HewlettPackard 3396 II integrator.

Samples were brought to room temperature if necessary, and $800 \mu \mathrm{L}$ was dispensed into a clean vial. The sample, and a small volume of $\mathrm{Q}-\mathrm{H}_{2} \mathrm{O}$ rinse, was taken up with a $1 \mathrm{~mL}$ Luer slip-tip syringe, which fit directly onto the three-way injection port (BioRad) and applied to the column. The laminarin eluted from the G-25 column in the void volume (between 21 and 29 minutes), and was reasonably well separated from the very broad salt peak which began to elute at 22 minutes, was significant at 29 minutes, and tailed out at 60 minutes. Laminarin concentrations less than $50 \%$ of the initial concentration $(<168 \mu \mathrm{g} / \mathrm{mL})$ were not resolved from the front of the salt peak, but could easily be detected by NMR analysis. For every sample, the 21-29 minute fraction was collected and lyophilized, redissolved in $0.5 \mathrm{~mL} \mathrm{D}_{2} \mathrm{O}$, and a proton spectrum was acquired. Each spectrum was collected using the same conditions: 1024 scans, using solvent suppression, with a receiver gain of 400 . All of the spectra were scaled to the zero-time spectrum, so that the decreases in peak height and area were proportional to the decrease in substrate concentration.

The five pullulan samples (collected at 40, 56, 60, 64, and 69 hours) run on the G25 column had the same elution profile as the laminarin samples. A complete set of pullulan samples was run on the G-50 column. As shown in Fig. 5.7, the pullulan eluted in the void volume, between 20 and 29 minutes (peak maximum: 23.3 minutes), while the salt peak from the medium began to elute at approximately 26 minutes, and became significant only at approximately 44 minutes (peak maximum: 57.6 minutes). Laminarin and maltoheptaose 
standards were also run to calibrate the column; laminarin eluted in a very broad peak that began with a very low shoulder at 21 minutes. The main peak extended from 29-61 minutes, with a peak maximum at 35 minutes. Maltoheptaose (MW 1153) eluted in a smooth broad peak beginning at 40 minutes and extending to 61 minutes, with a peak maximum at 51 minutes. A mixture of pullulan, laminarin, and maltoheptaose standards injected on the G-50 column was resolved into three distinct, overlapping peaks, with peak maxima at 23,35 , and 51 minutes. Based on these tests, three fractions were collected for the pullulan samples: 20-29 minutes, 29-42 minutes, and 42-68 minutes.

The fractions were individually lyophilized and redissolved in $0.5 \mathrm{~mL} \mathrm{D}_{2} \mathrm{O}$. Proton spectra were acquired for all pullulan fractions of all time points. Spectra of the first two fractions were acquired under the same conditions as for laminarin (1024 scans, $R G=400$, solvent suppresion). Since the third fraction contained nearly all of the salt and all of the buffer from the medium, the maximum receiver gain setting which could be used was 20; and 2048 scans were acquired. All of the spectra of the first two fractions from every time point were autoscaled to the zero-time spectrum of the first fraction, so that increases and decreases in peak height and area should be proportional to changes in substrate concentration. Because the third fraction contained elevated carbon levels (buffer) and salt and was acquired under different spectrometer conditions, it was scaled by visual inspection to the same signal/noise level as the first two fractions.

The carbohydrates from Synechococcus WH7335 were initially obtained and characterized as described in Chapter 3. Samples of F2 were run on the G-25 column and collected as for laminarin. Long-term ( $>50,000$ scans, acquisition time $\sim 100$ hours) ${ }^{1} \mathrm{H}$ NMR spectra were acquired to determine the presence of $F 2$ in the culture medium. Since F3 eluted in the same fraction as the salts and buffer in the culture medium, the F3 samples were lyophilized and NMR spectra were acquired without attempting to size fractionate or remove salts on a gel column.

\section{Results and Discussion}

Reproducibility and general results

Reproducibility among replicate vials was excellent (see Chapter 4 for discussion), and the general characteristics of the cultures (headspace gas profiles, $\mathrm{pH}$, evolution of total $\mathrm{CO}_{2}$ ) were very similar to the di- and oligosaccharide cultures described in Chapter 4. All of the substrates were rapidly degraded during the first 70 hours of the cultures. Because sampling intervals were closely spaced, however, it was possible to determine that the substrates differed from one another in the time scale of their removal from the cultures. 
Headspace gas profiles followed the pattern of the previous cultures, with early production and swift consumption of $\mathrm{H}_{2}$ (Fig. 5.1; note that headspace gas samples were collected at less-frequent intervals than medium samples), a gradual increase in headspace $\mathrm{CO}_{2}$ over the first several days of the culture followed by a sharp increase (Fig. 5.2) at later time points, low levels of $\mathrm{CH}_{4}$ (not shown), and appearance of $\mathrm{H}_{2} \mathrm{~S}$ at the later phases of the cultures. Recovery of laminarin and pullulan carbon as $\mathrm{CO}_{2}$ after 26 days averaged $60 \%$ (see Fig. 5.2), similar to carbon recoveries for the di- and oligosaccharide cultures (Chapter 4). Recovery of carbon for F3 can only be estimated, since the exact carbon content of the substrate as added to the culture is not well constrained. Using the $10 \%$ carbohydrate composition estimated for F3 (i.e., $5 \mathrm{mg}$ of substrate), recovery of carbon as $\mathrm{CO}_{2}$ is $61.7 \%$; if $\mathrm{F} 3$ is $50 \%$ carbohydrates, carbon recovery would be $12.3 \%$. As described below, NMR analysis of the F3 culture showed that substrate consumption was complete. Either the carbon assimilation efficiency for bacteria consuming the teichoic acidtype polysaccharide is higher than for bacteria consuming the other carbohydrates used in the cultures (i.e., more than 10-30\% of substrate carbon is assimilated as cellular carbon), or the carbohydrate concentration of $\mathrm{F} 3$ as used in the culture was closer to $10 \%$ than $50 \%$.

The $\mathrm{F} 2$ culture showed a very slight increase in $\mathrm{CO}_{2}$ over the course of the culture series, but total $\mathrm{CO}_{2}$ levels were not significantly above the blank levels at any time. Total substrate concentration was only $56 \mu \mathrm{g}$, so the lack of measurable difference between F2 and blank cultures is not surprising. The carbon content of $\mathrm{F} 2$ is $40 \%$; if $100 \%$ of $\mathrm{F} 2$ were converted to $\mathrm{CO}_{2}$, the increase in total $\mathrm{CO}_{2}$ would be only $1.8 \mu \mathrm{mol}$. Several pieces of evidence suggest that $\mathrm{F} 2$ was consumed during the course of the culture: the headspace gas of the $\mathrm{F} 2$ culture contained a trace of $\mathrm{H}_{2}$ at 49.5 hours, and a small quantity of $\mathrm{H}_{2} \mathrm{~S}$ was measured in the total $\mathrm{CO}_{2}$ determination at 711 hours. None of the culture blanks produced measurable $\mathrm{H}_{2}$ or $\mathrm{H}_{2} \mathrm{~S}$. In addition, as discussed below, NMR spectra showed that $\mathrm{F} 2$ disappeared from the medium over the course of the experiment.

As in the oligosaccharide cultures, there was a significant time interval between the complete degradation of carbohydrate substrate and the maximum $\mathrm{CO}_{2}$ levels in the cultures, a reasonable result since carbon dioxide is one of several products of the initial steps in carbohydrate fermentation (Wood, 1961; Gottschalk, 1986). As shown in the degradation of cellulose by pure cultures of rumen bacteria, other products such as low molecular weight acids and alcohols are also present in significant quantities (Weimer and Zeikus, 1977). Only when these substrates are converted to $\mathrm{CO}_{2}$ by secondary degraders do the $\mathrm{CO}_{2}$ levels in the cultures reach a maximum. The $\mathrm{pH}$ levels of the cultures dipped very slightly, even less than in the case of the di- and oligosaccharide cultures, and then returned to starting values during the 26-day course of the experiment; either the turnover 


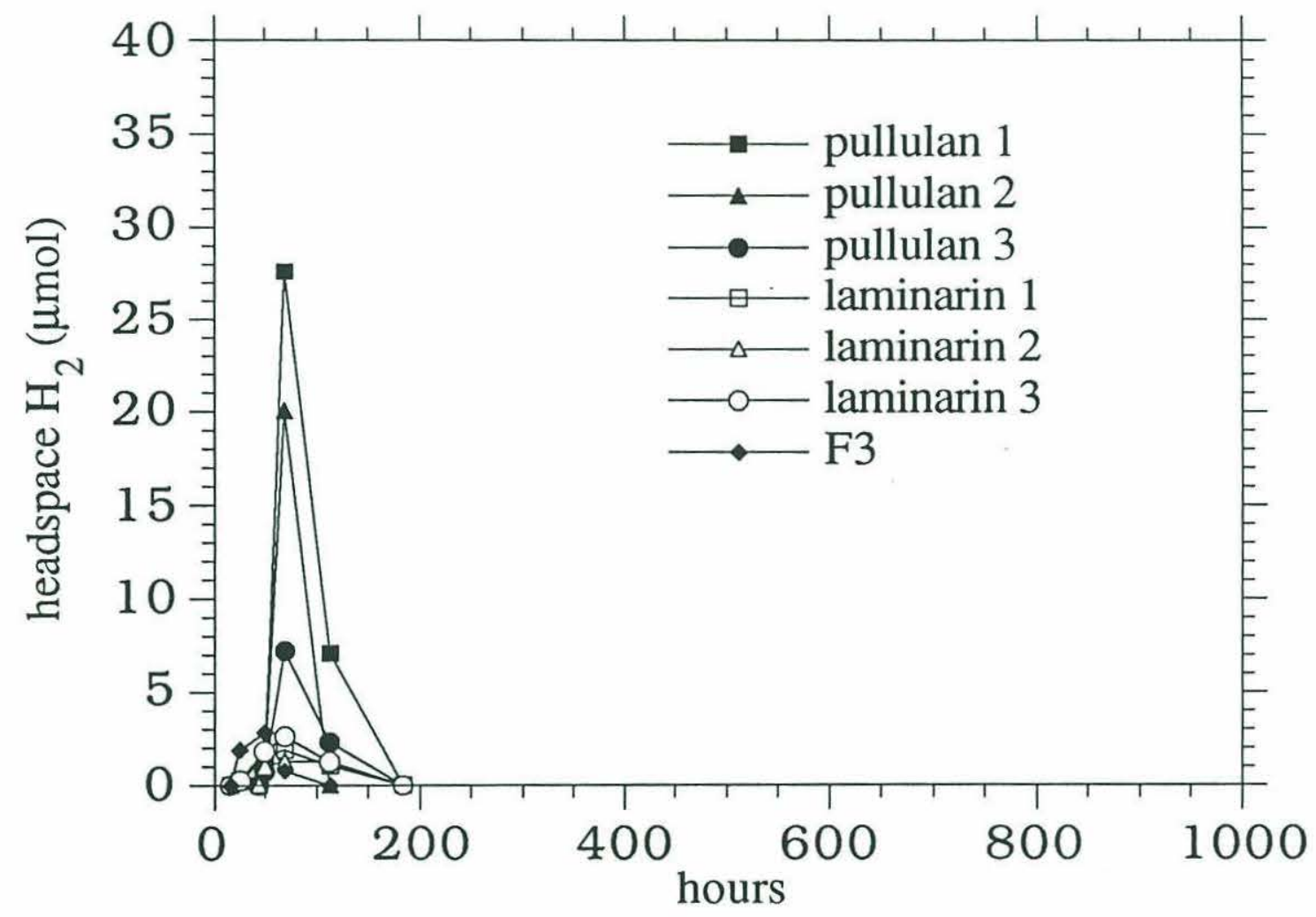

Fig. 5.1

Production and consumption of headspace $\mathrm{H}_{2}$ for all cultures except $\mathrm{F} 2$, which produced only a trace of $\mathrm{H}_{2}$. 


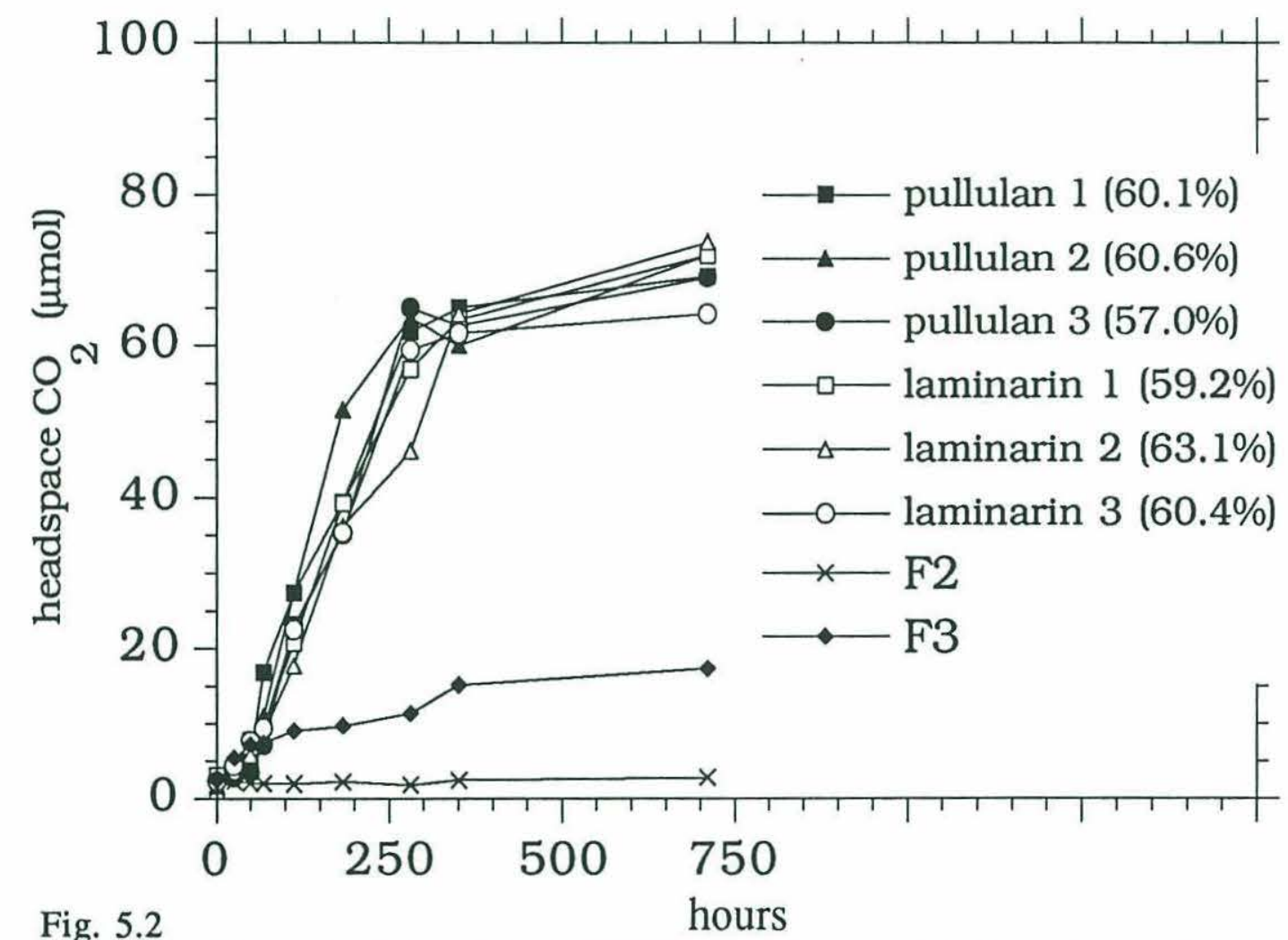

Production of headspace $\mathrm{CO}_{2}$ for all cultures. Recovery (at 711 hours) of carbon as $\mathrm{CO}_{2}$ for all cultures except F2 and F3 is given in brackets (as \% of substrate carbon). Note that initial substrate concentrations for $\mathrm{F} 2$ and $\mathrm{F} 3$ were lower than for the other cultures. 
time of organic acids was faster than in the oligosaccharide cultures, or fewer acidic intermediates were produced.

The blank vials (B, I, and IAS) all had headspace $\mathrm{CO}_{2}$ levels $\leq 5 \mu \mathrm{mol}$, and produced no $\mathrm{H}_{2}, \mathrm{CH}_{4}$, or $\mathrm{H}_{2} \mathrm{~S}$. Total $\mathrm{CO}_{2}$ levels in the blanks ranged from 16-35 $\mu \mathrm{mol}$, equivalent to $4-9 \%$ of the total $\mathrm{CO}_{2}$ in the laminarin and pullulan cultures. The F3 IAS total $\mathrm{CO}_{2}$ level was $10 \mu \mathrm{mol}$, equivalent to $9.7 \%$ of the level in the $\mathrm{F} 3$ culture. Recovery of substrate from the B and IAS vials was $100 \%$ on the 26th day of the culture series, demonstrating that substrate was not lost through sorption to the walls of the vials or to sediment particles.

\section{Substrate degradation}

Degradation of substrate is summarized in Fig. 5.3, which shows the F3, laminarin, and pullulan profiles. Because of the small volume of the culture (11 mL), F2 was measured only at two timepoints, 0 and 69 hours, which are represented by filled circles in Fig. 5.3. At each timepoint, $1.5 \mathrm{~mL}$ (instead of $1.0 \mathrm{~mL}$ ) of medium was removed; removal of additional medium at intermediate timepoints would have excessively drained the culture.

F2 concentrations were determined by examining the NMR spectra of the medium removed at 0 and 69 hours (Fig. 5.4). A long-term ( $>50,000$ scans, acquisition time $\sim 120$ hours) ${ }^{1} \mathrm{H}$ NMR spectrum of the zero hour sample clearly showed the presence of anomeric protons at $\delta=5.2$ and 5.4 , consistent with the NMR spectrum of F2 where these two resonances corresponded to a free $\alpha$ anomer and the $\alpha(1,4)$ linkage, respectively (Fig. 3.8). The anomeric resonance at $\delta=4.95$ corresponding to the $\alpha(1,6)$ linkage was obscured by spinning sidebands from the HOD (solvent) resonance, but the other anomeric resonances were clearly discernible. The quality of the spectrum is quite good, considering that the zero-time sample contained only $7.5 \mu \mathrm{g}$ of a 5000 dalton polysaccharide (from 1.5 $\mathrm{mL}$ medium). An additional fraction of the F2 sample was collected from the G-25 GPC column (29-31 minutes) to check for lower molecular weight hydrolysis products; the NMR spectrum did not show any anomeric proton resonances.

F3 concentrations were determined by measuring the height of the anomeric resonance at $\delta=5.12$ of the corresponding NMR spectra, whereas laminarin and pullulan concentrations were calculated from GPC analysis of samples and integration of peak areas as determined by refractive index detection. The data for pullulan were corrected for a highmolecular weight contaminant which co-eluted with pullulan; the area of the contaminant material represented approximately $3 \%$ of the total peak area of the original pullulan concentration. The correction was made by subtracting the peak area of the contaminant 


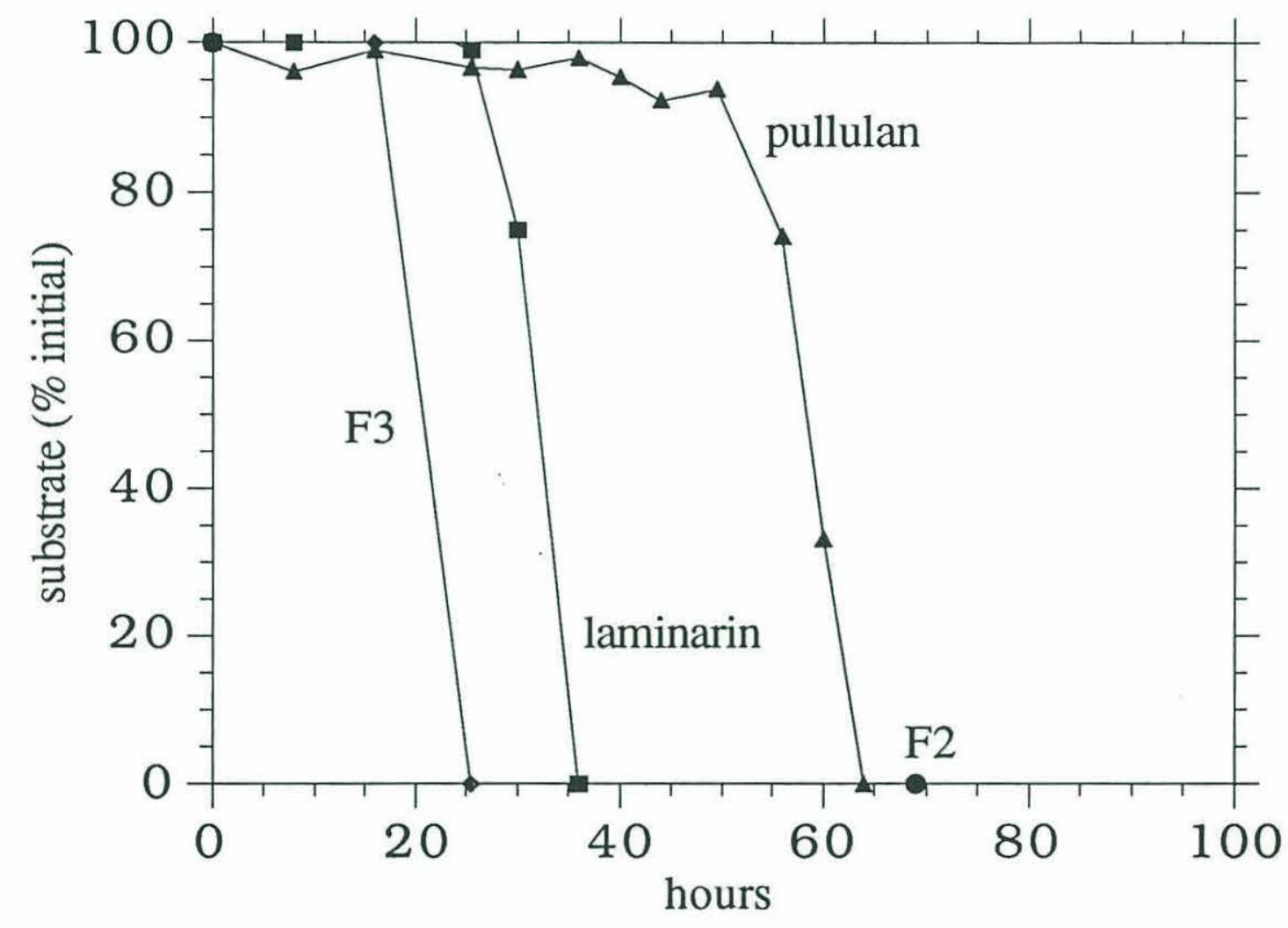

Fig. 5.3

Time-course of F2, F3, pullulan, and laminarin degradation. 

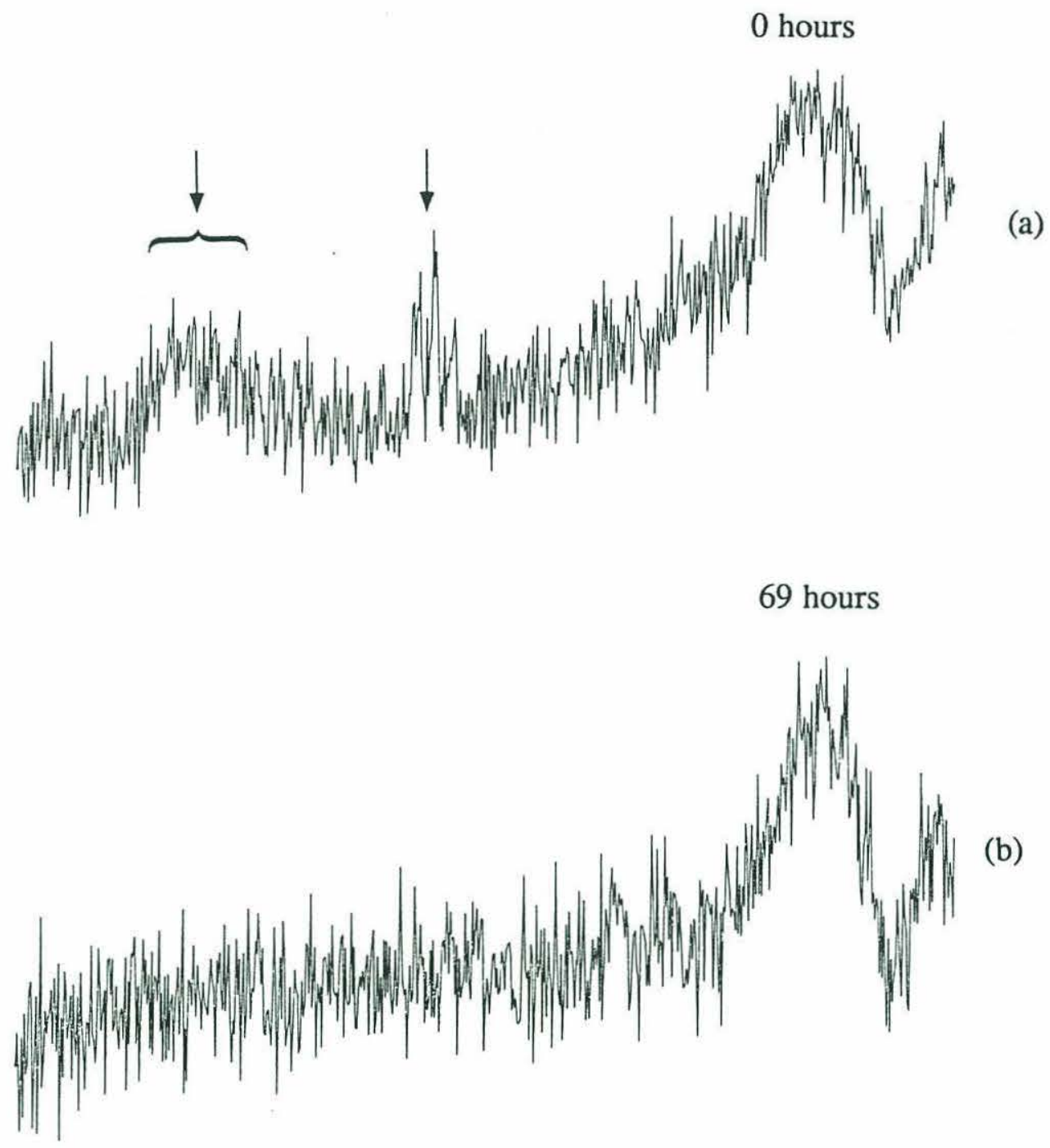

(b)

Fig. 5.4

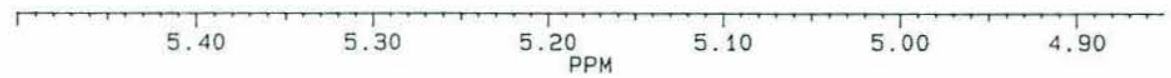

${ }^{1} \mathrm{H}$ NMR spectrum of anomeric region of F2 at zero-time (a) and 69 hours (b). Anomeric resonances are marked with arrows. 
(as measured in a sample collected at 447 hours) from all peak areas for previous time points. In the NMR spectra of the pullulan samples, the contaminant corresponded to a poorly-defined, broad resonance in the high-field region $(\delta=0.9-1.1)$. The same contaminant was observed in the NMR spectra of the laminarin samples, but the data were not corrected because a peak area count for the contaminant could not be determined after complete degradation of laminarin, since the co-eluting lead edge of the salt peak obscured the contaminant (see previous comments about the G-25 column).

The F3 samples were lyophilized directly, because GPC could not separate the substrate from the medium salts. The F3 NMR spectra were therefore dominated by resonances belonging to the buffer (HEPES), and suffered in quality from the high concentrations of salts (ringing near the HOD peak, prominant spinning sidebands). Nonetheless, the anomeric regions of the spectra were well resolved, and changes in the anomeric resonances were easily observed. Fig. 5.5 shows the anomeric region of the NMR spectra of F3 for the first 25.5 hours of the culture series. From top to bottom, the spectra are of samples collected at $0,8,16$, and 25.5 hours. The anomeric proton at $\delta=5.12$ is the major anomer from F3, corresponding to terminal glucose, and the anomer at $\delta=5.17$ corresponds to terminal galactose. The less well-defined anomeric resonance at $\delta=5.40$ probably represents 4-linked glucose (see Chapter 3 ). The relative intensity of the anomer at $\delta=5.4$ appeared to decrease slightly over the first 16 hours, whereas the major F3 anomer appeared unchanged over the same time period, and then abruptly disappeared (along with the remainder of the anomer at $\delta=5.4$, and the low-intensity $\alpha$-anomers at $\delta=5.18$ and 5.22) between 16 and 25.5 hours. The slight change in the intensity of the anomeric resonance at $\delta=5.40$ may indicate that $\mathrm{F} 3$ is preferentially hydrolyzed at the phosphoric diester-glucosephosphoric diester bonds; higher resolution NMR spectra (longer-term acquisition) would help resolve the change. (3000-scan spectra (ca. 6 1/2 hours) were acquired for Fig. 5.5.) The onset of degradation may therefore be more rapid than is shown in Fig. 5.3, for which only the changes in the major anomeric resonance at $\delta=5.12$ were measured.

Laminarin was not degraded as quickly as F3, but the transition from substantial quantities of substrate to complete removal was equally swift. Figure 5.6 shows the anomeric region of the NMR spectra of the laminarin fraction collected from the G-25 column. These samples were collected during the first 36 hours of the culture series. The anomeric resonance at $\delta=4.79$ appears unchanged from 0 to 25.5 hours (Figs. 5.6 a-d). At 30 hours (Fig. 5.6e), the resonance is noticeably smaller, and there is a slight indication of a free $\alpha$ anomer at $\delta=5.2$. Twenty-five percent of the laminarin was degraded at this time (Fig. 5.3). No anomeric resonances are observed at 36 hours (Fig. 5.10f); all of the 

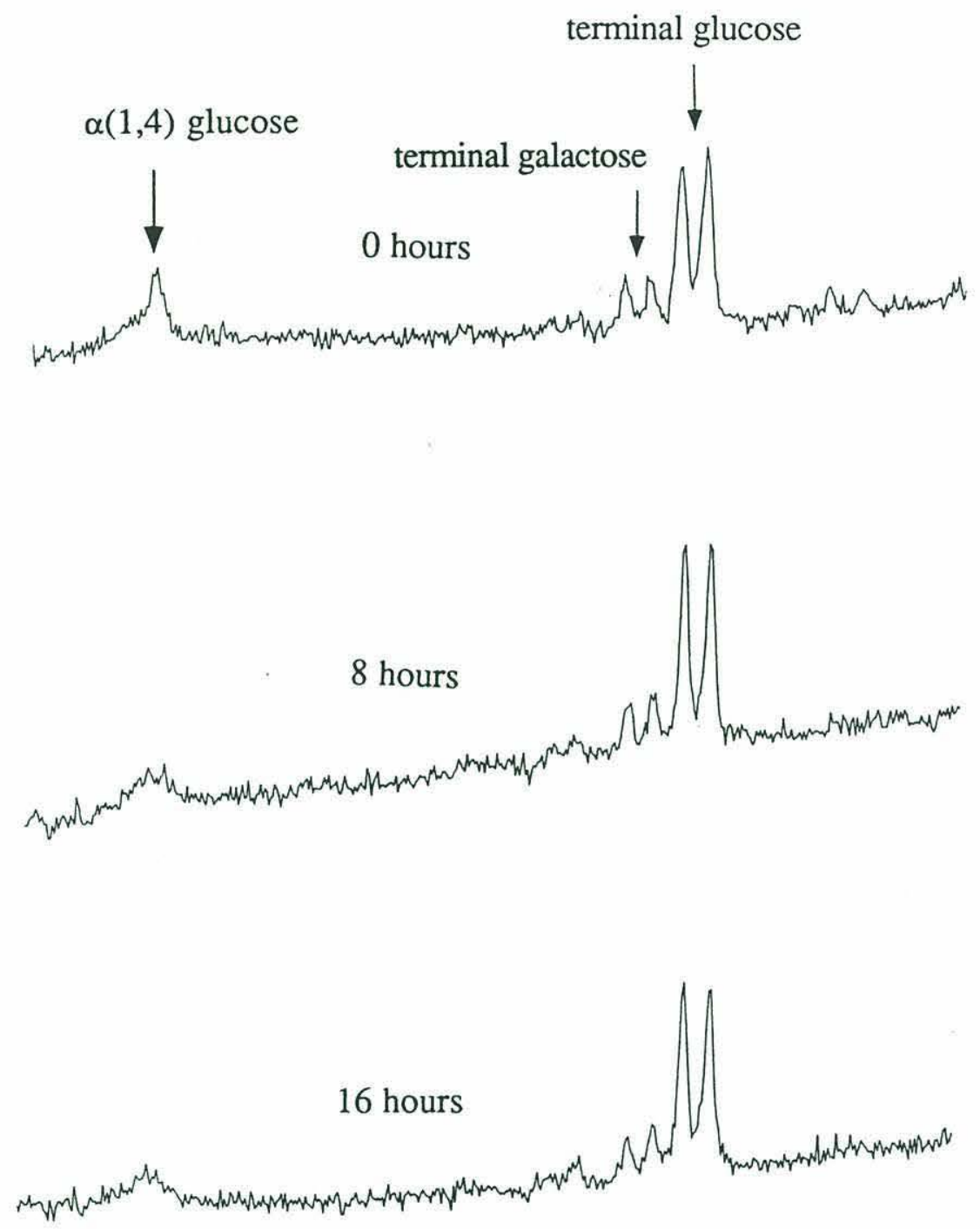

(c)

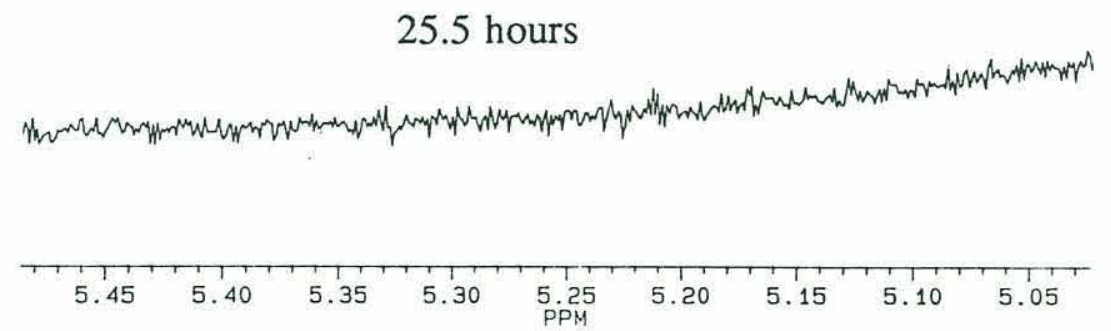

Fig. 5.5

${ }^{1} \mathrm{H}$ NMR spectrum of anomeric region of F3 at zero-time (a), 8 hours (b), 16 hours (c), and 25.5 hours (d). Anomeric resonances marked with arrows. 

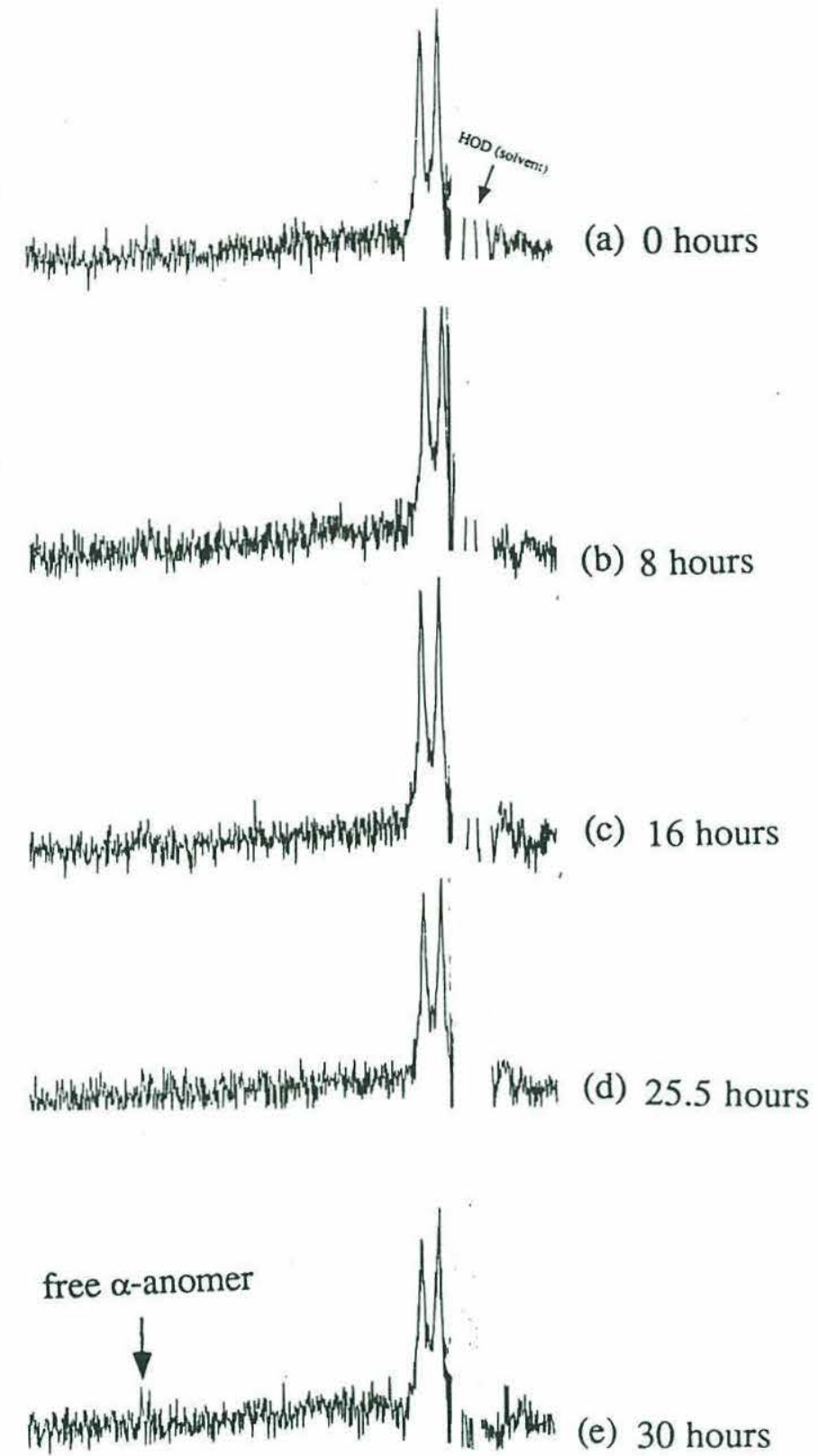

Fig. 5.6

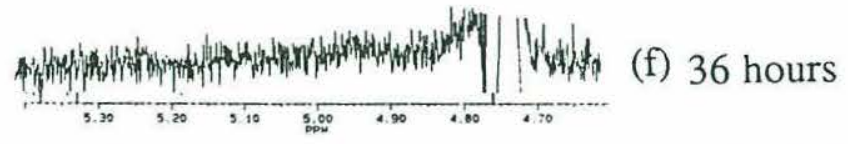

${ }^{1} \mathrm{H}$ NMR spectrum of anomeric region of laminarin at zero-time (a), 8 hours (b), 16 hours (c), 25.5 hours (d), 30 hours (e), and 36 hours (f). Anomeric resonances marked with arrows. 
laminarin was degraded within the first 36 hours of inoculation, with the greatest change occurring between 30 and 36 hours.

Pullulan was degraded slightly more slowly than either F3 or laminarin, although like the other substrates, the transition from high concentration to complete degradation of initial substrate occurred very swiftly. In the case of pullulan, degradation occurred over a span of 14 hours. The lag before the onset of rapid substrate degradation is expected, because the sediment inoculum contained a range of bacterial types. The primary fermenting bacteria responsible for the initial steps of degradation require time to double sufficiently to degrade the substrate; the amount of time is a function of initial bacterial cell numbers and doubling time. The length of the initial lag $(16,25.5$, and 49.5 hours for F3, laminarin, and pullulan, respectively) is consistent with growth of a number of generations of bacteria, since bacterial doubling times are often on the order of hours to tens of hours (Gottschalk, 1986). Even in pure- and co-cultures of bacteria, where the initial bacterial inoculum is often orders of magnitude higher than in these polysaccharide cultures, lags are often observed between inoculation and the onset of substrate degradation. A 17-hour lag was observed, for example, before initial degradation of cellulose by a pure culture of Clostridium thermocellum (Weimer and Zeikus, 1977). In pure cultures of several species of rumen bacteria, a 2-3 hour lag was observed before the onset of starch degradation (Cotta, 1992).

The lag before onset of rapid substrate degradation was shortest for F3 (16 hours), followed by laminarin ( 25.5 hours), and pullulan ( 49.5 hours), but once rapid degradation began, the time interval for degradation of initial substrate was very similar $(9.5,10.5$, and 14 hours (Fig. 5.3); note that zero-concentration samples may have been collected several hours after the substrate was completely removed.) Extracellular hydrolysis per se does not appear to be the slow factor in degradation of teichoic acid-type or glucose polysaccharides. This is similar to results from a study of the degradation of cellodextrins (DP1-7 $\beta(1,4)$ linked glucose oligosaccharides) by pure cultures of rumen bacteria. Although cellopentaose and cellohexaose were hydrolyzed extracellularly prior to uptake, hydrolysis was not a slow step in the degradation process (Russell, 1985). The exceptionally swift degradation of teichoic acid-type polysaccharide may indicate that phosphoric diesters are particularly easily hydrolyzed, and that a large percentage of the bacterial population in the initial inocculum has the enzymatic capability to hydrolyze these bonds. 


\section{Detailed Analysis of Pullulan Degradation}

The G-50 column used for the pullulan samples clearly resolved the changes in the pullulan substrate through the course of the culture. Figs. 5.7-5.11 show the refractive index detector plot of substrate (and salt) for $0,49.5,56,60$, and 64 hours, respectively. (Note that these data are further shown in Figs. 5.12-5.18, and are summarized in Fig. 5.19). The zero time plot (Fig. 5.7) showed a well-resolved pullulan peak starting at 20.4 minutes, with a peak maximum at 23.5 minutes, corresponding to a refractive index maximum of 10.2. At 49.5 hours, the refractive index maximum had decreased to 9.4 (Fig. 5.8), and by 56 hours (Fig. 5.10), to 7.6. At 56 hours, the refractive index between 29 and 42 minutes had also increased by 0.2 refractive index units, suggesting that greater quantities of material were eluting just behind the void volume of the column. These changes became even more pronounced at 60 hours (Fig. 5.10). The lead edge of the peak appeared slightly later (21.1 minutes), the peak maximum was shifted back to 23.9 minutes and had a refractive index value of just 2.8. In addition, the refractive index between 28 and 42 minutes was higher than observed for any previous sample, which implied that relatively large quantities of material were below the nominal exclusion limit of 10,000 daltons. By 64 hours (Fig. 5.11), the void volume peak had been reduced even further; subsequent NMR analysis showed that this material was the high molecular weight, high NMR field contaminant, and did not contain any pullulan. (The same peak was observed in samples collected at later time points as well.) The refractive index between 29 and 42 minutes was still slightly higher than observed for the zero time point (Fig. 5.7), which again suggested that material was eluting just behind the void volume of the column.

GPC and NMR analysis of the samples provided excellent size and structural resolution of the substrate and its degradation products. Three distinct molecular weight classes of pullulan and its hydrolysis products, $\geq 10,000$ daltons (20-29 minutes), $\sim 5000$ daltons (29-42 minutes), and $\leq \sim 1200$ daltons (42-68 minutes), were resolved. Since pullulan contains both $\alpha(1,4)$ and $\alpha(1,6)$ linkages, there are several possible means by which it could be hydrolyzed to smaller poly- and oligosaccharides. NMR analysis of the size-fractionated samples showed that one linkage type was preferentially hydrolyzed.

Figs. 5.12-5.18 show the anomeric region of the NMR spectra of time points between inoculation ( 0 hours, Fig. 5.12) and complete degradation of pullulan and its lower molecular weight hydrolysis products at 69 hours (Fig. 5.18). In all of the figures, the top spectrum is the fraction corresponding to the void volume of the column, which should contain any material with a nominal molecular weight $\geq 10,000$ daltons. The middle spectrum is the fraction corresponding to the elution of laminarin (5,000-6,000 daltons), 


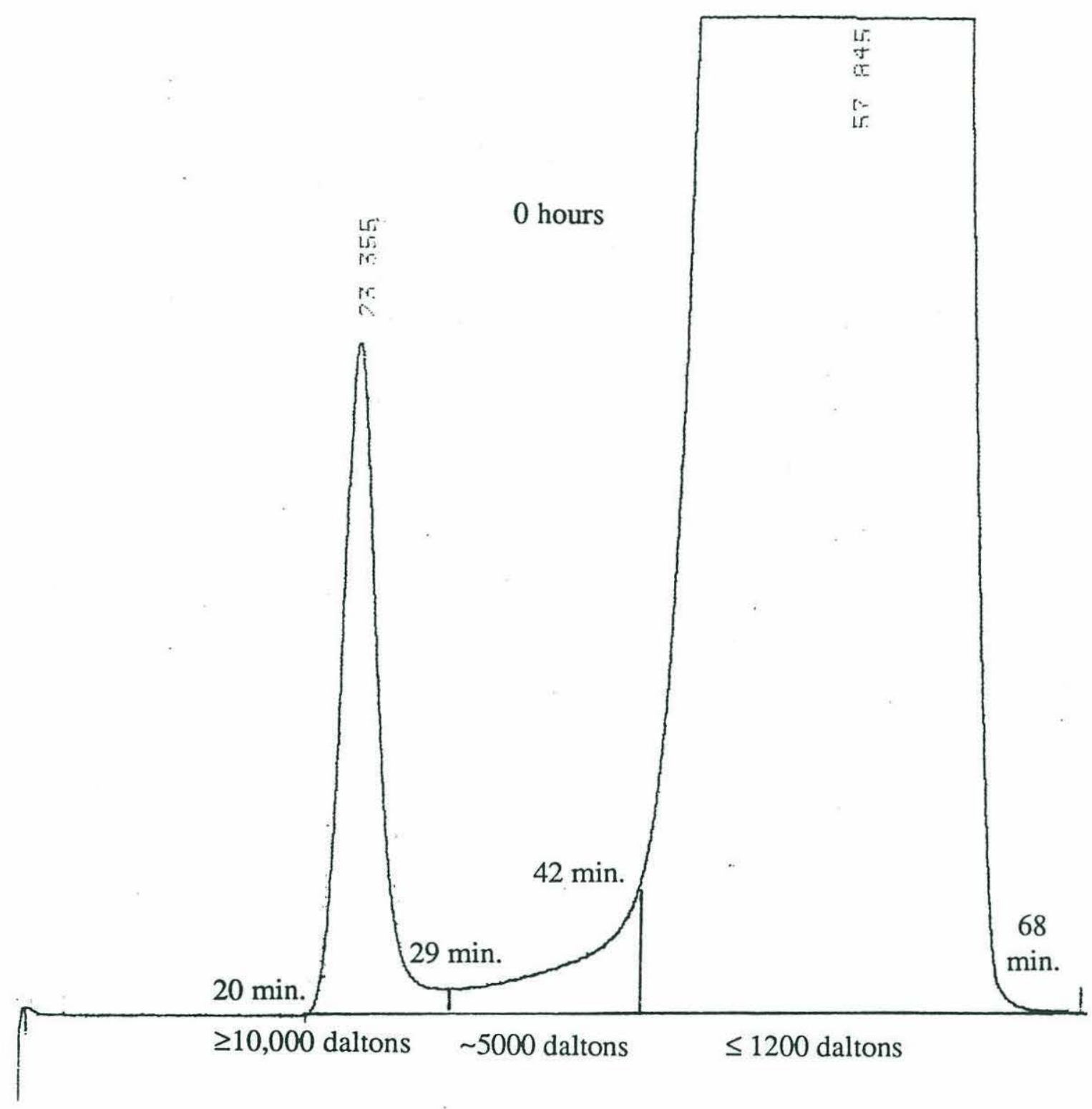

Fig. 5.7

G50 gel permeation chromatogram of pullulan (zero hours). $\geq 10,000$ dalton (20-29 minutes), $\sim 5000$ dalton (29-42 minutes), and $\leq 1200$ dalton (42-68 minutes) fractions were collected as marked. (See text for chromatography specifications.) 


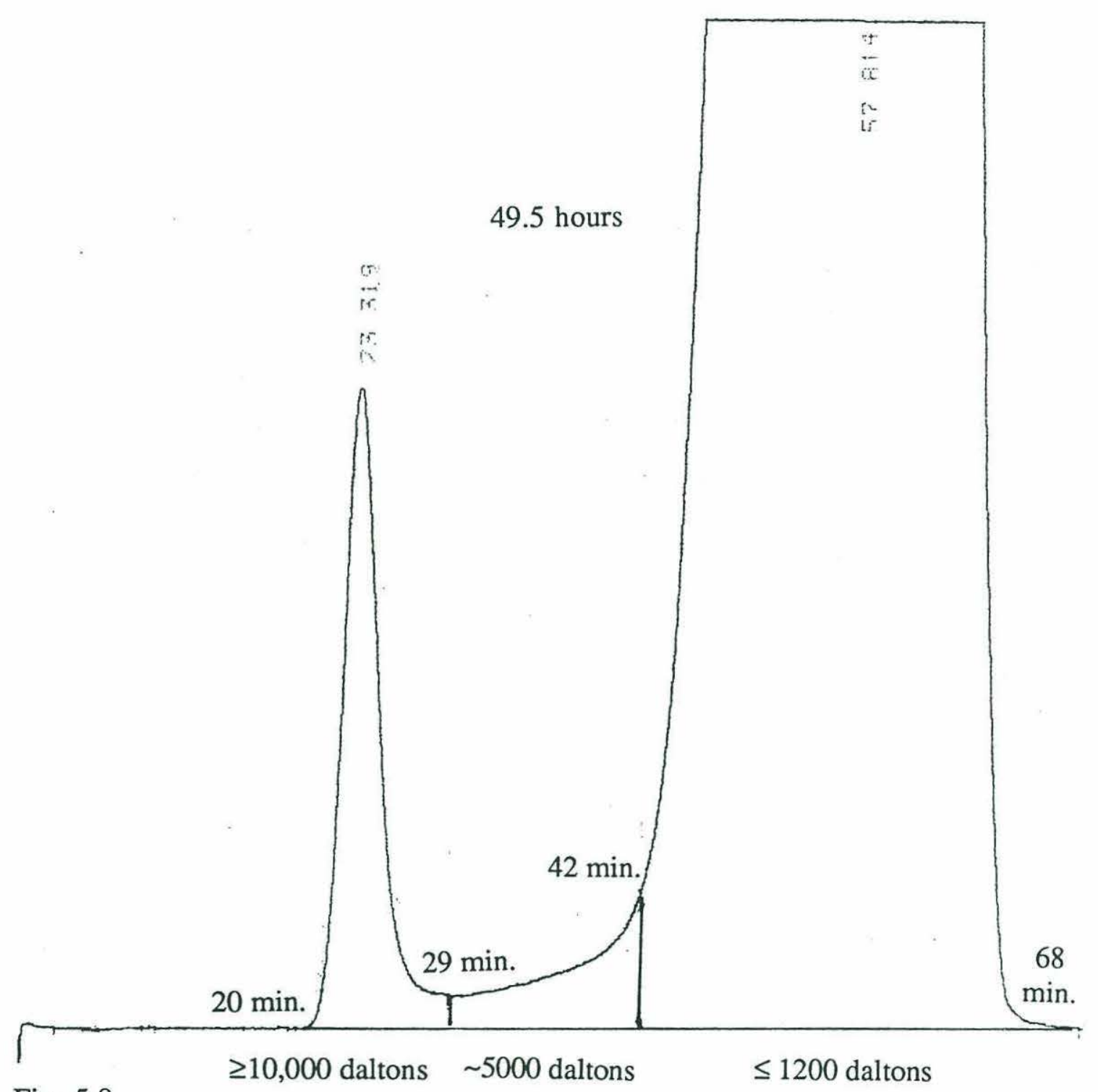

Fig. 5.8

G50 gel permeation chromatogram of pullulan (49.5 hours). $\geq 10,000$ dalton (20-29 minutes), $~ 5000$ dalton (29-42 minutes), and $\leq 1200$ dalton (42-68 minutes) fractions were collected as marked. (See text for chromatography specifications.) 


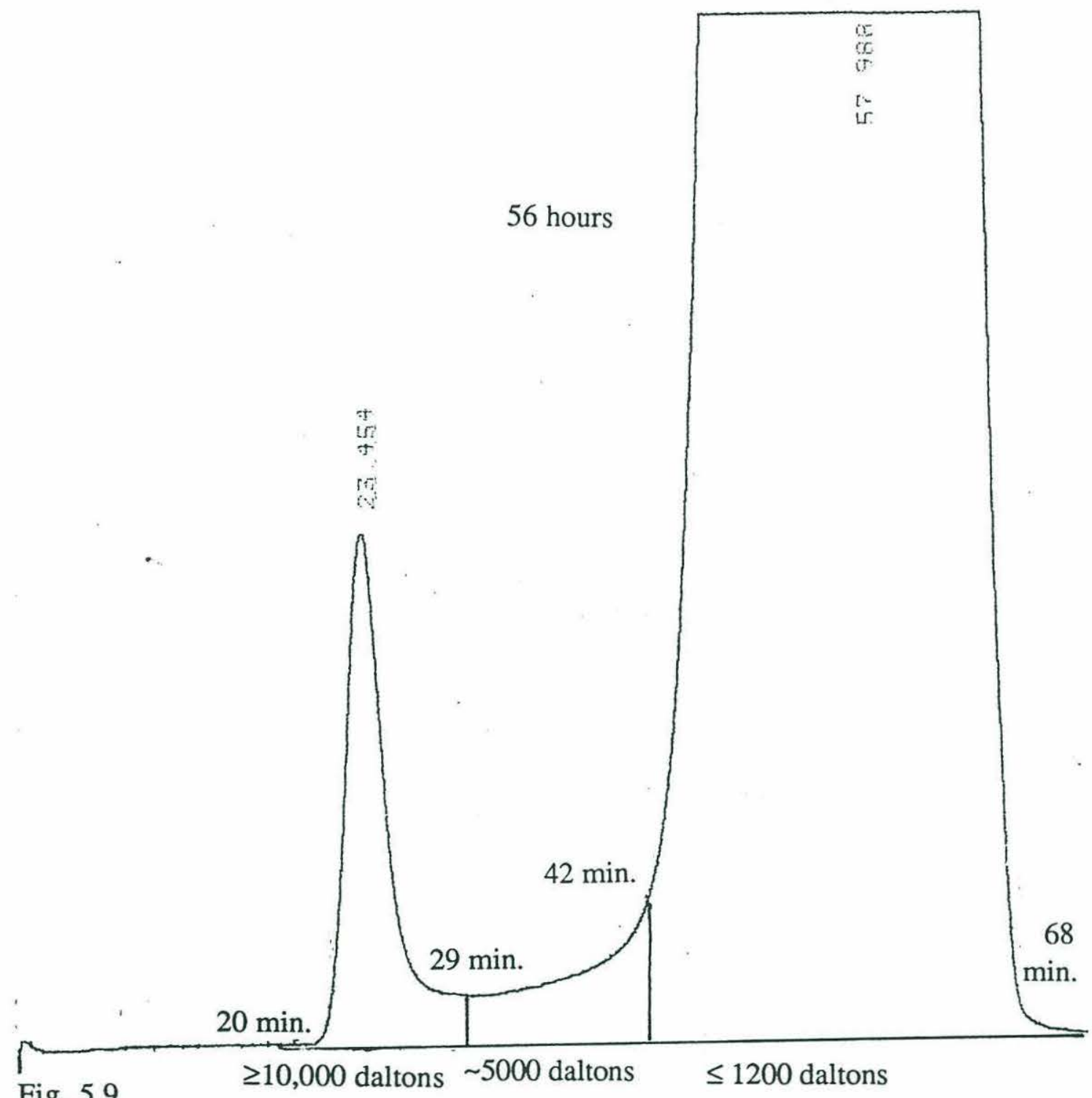

Fig. 5.9

G50 gel permeation chromatogram of pullulan (56 hours). $\geq 10,000$ dalton (20-29 minutes), $~ 5000$ dalton (29-42 minutes), and $\leq 1200$ dalton (42-68 minutes) fractions were collected as marked. (See text for chromatography specifications.) 


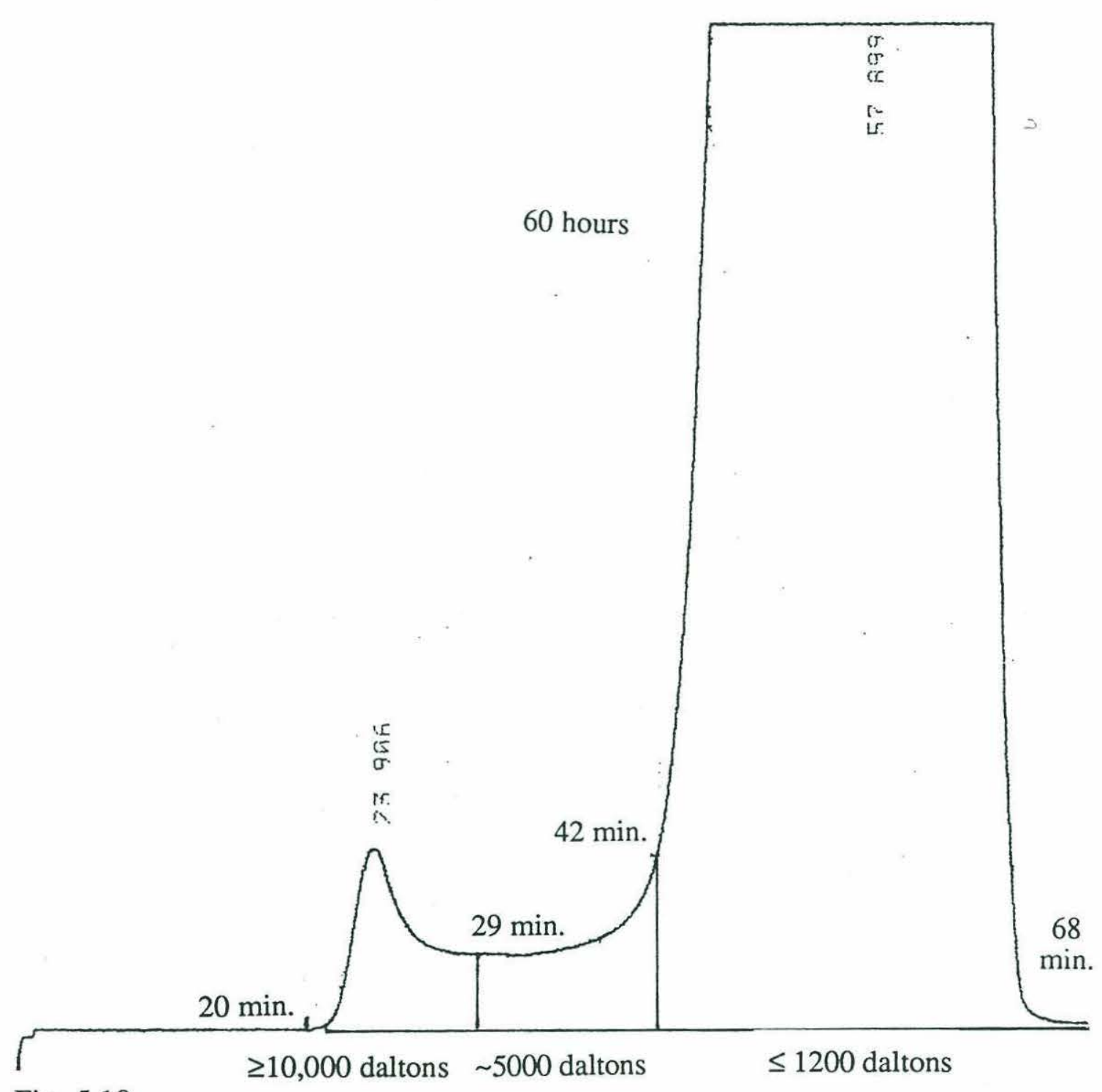

Fig. 5.10

G50 gel permeation chromatogram of pullulan ( 60 hours). $\geq 10,000$ dalton (20-29 minutes), $\sim 5000$ dalton (29-42 minutes), and $\leq 1200$ dalton (42-68 minutes) fractions were collected as marked. (See text for chromatography specifications.) 


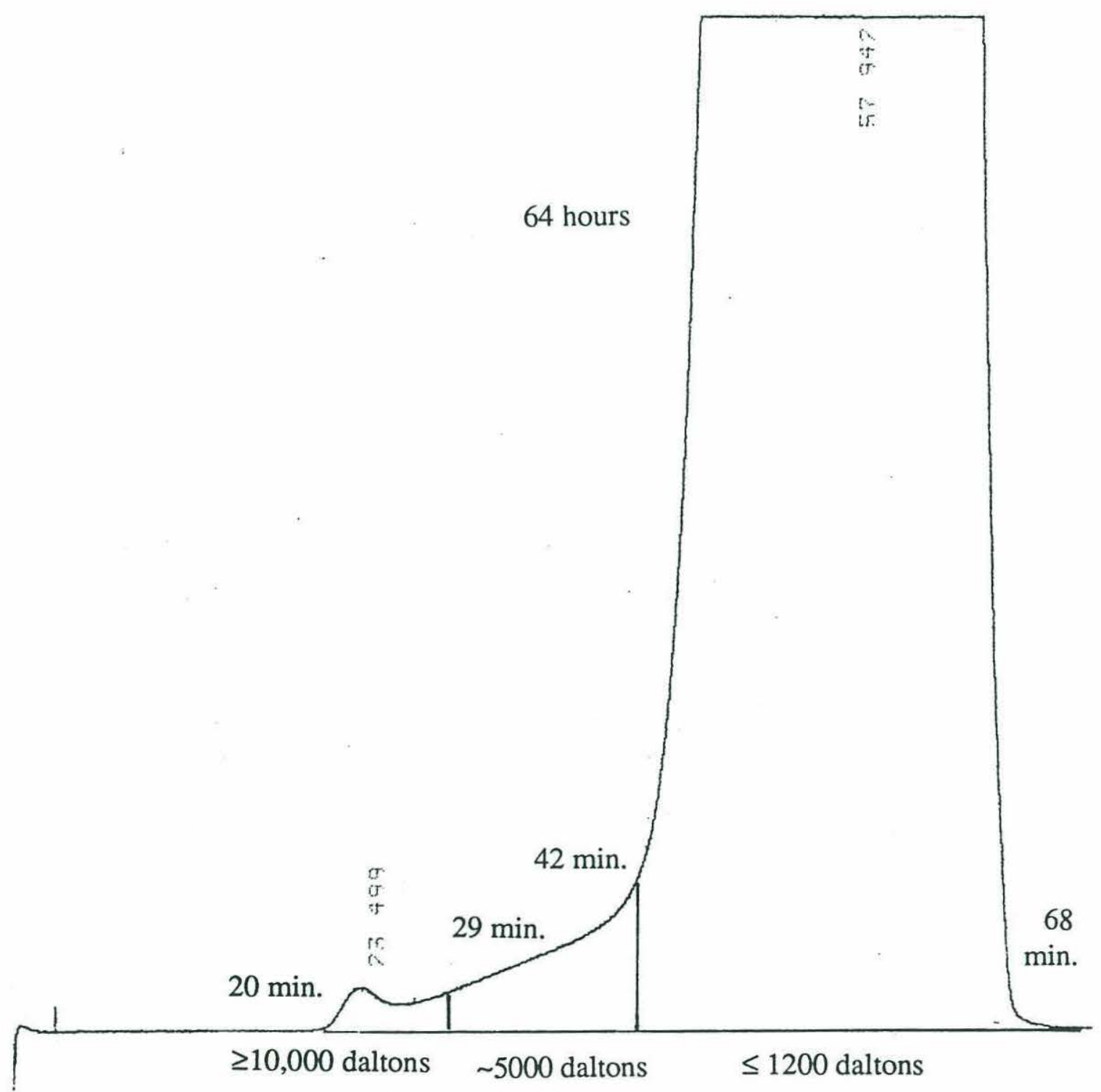

Fig. 5.11

G50 gel permeation chromatogram of pullulan ( 64 hours). $\geq 10,000$ dalton (20-29 minutes), $~ 5000$ dalton (29-42 minutes), and $\leq 1200$ dalton (42-68 minutes) fractions were collected as marked. (See text for chromatography specifications.) 
and the bottom spectrum is the fraction which corresponded to maltoheptaose $(\leq \sim 1200$ daltons). In the $\geq 10,000$ dalton MW fraction of the zero-time sample, there are three wellresolved anomeric resonances at $\delta=4.95,5.36$, and 5.40, corresponding to the $\alpha(1,6)$ linkage and the two $\alpha(1,4)$ linkages of the maltotriose units, respectively. The 5,000 dalton fraction (Fig. 5.12b) has no distinctive features, while the 1200 dalton fraction (Fig. 5.12c) shows a low increase centered around $\delta=5.4$ and perhaps a slight indication of a peak near $\delta=5.2$, suggesting that the pullulan substrate may contain low levels of maltose and/or maltotriose. Spectra of samples collected during the first 36 hours were similar to the zerotime samples; only $2 \%$ of the pullulan was degraded during this time interval (Fig. 5.3). The sample collected at 40 hours (Fig. 5.13), when approximately $5 \%$ of the pullulan had been degraded (Fig. 5.3), began to show changes. In Fig. 5.13, slight indications of peaks around $\delta=5.4$ and 4.95 suggest that some of the original 200,000 -dalton pullulan has been hydrolyzed to a molecular weight of 5000-6000 daltons. These peaks in the 5000-6000 dalton range became much more distinct through the remaining time points (Figs. 5.145.18 ) ; at 56 hours (Fig. 5.15), with $25 \%$ of the original pullulan no longer in the $\geq 10,000$ dalton fraction, even the J-coupling of the anomeric resonances was resolved.

In a similar fashion, the progressive increase in anomeric resonances in the 1000 dalton molecular weight class was apparent through the time points at 49-64 hours (Figs 5.14-5.17). The changes between 60 and 69 hours (Figs. 5.16-5.18), when pullulan in the molecular weight class $\geq 10,000$ daltons was reduced from $33 \%$ of starting concentration to $0 \%$, are especially significant. In Fig. 5.16a, the decrease in peak height of the $\geq 10,000$ dalton pullulan is clearly evident. A relatively large amount of material, with well-resolved anomeric resonances, is in the 5000 dalton size fraction (Fig. 5.16b), and Fig. 5.16c shows substantial anomeric resonances in the 1200 dalton molecular weight range. Fig. 5.17 a shows that at 64 hours, all of the pullulan substrate had been removed from the $\geq 10,000$ dalton fraction; only substrate in the 5000 and 1200 dalton fractions remained (Figs. 5.17b and c). Just 5 hours later, at 69 hours, all of the carbohydrate in all size classes has been consumed (Figs. 5.18a-c).

The changes in distribution of pullulan among the size classes are summarized in Fig.5.19. The areas of the anomeric resonances in each size fraction at each time point were integrated and plotted. (Note that scaling of the NMR spectra for the 1200 dalton size fraction was done by visual inspection; error in the scaling is propagated in the plot.) Figure 5.20 shows a bar graph of the same data, which shows that onset of hydrolysis was far more rapid than removal of substrate from the medium. At 60 hours, even though only $33 \%$ of the pullulan remained in the $\geq 10,000$ dalton size class, more than $80 \%$ of the 


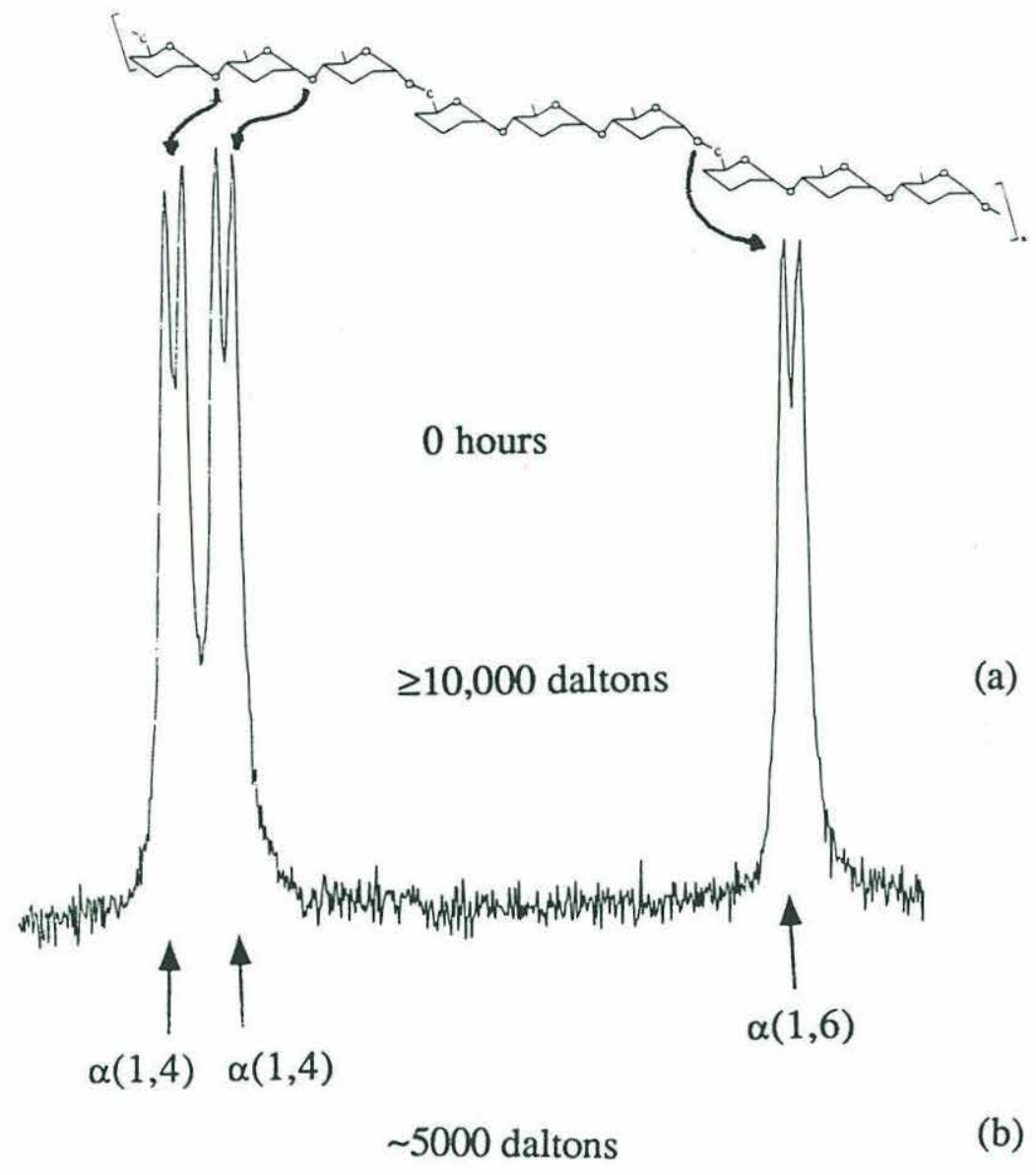

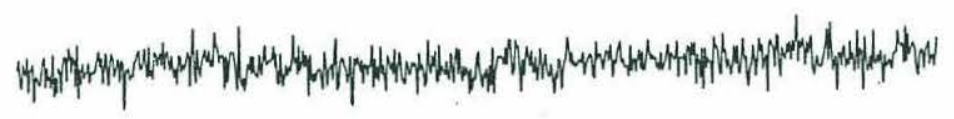

$$
\leq 1200 \text { daltons }
$$

(c)

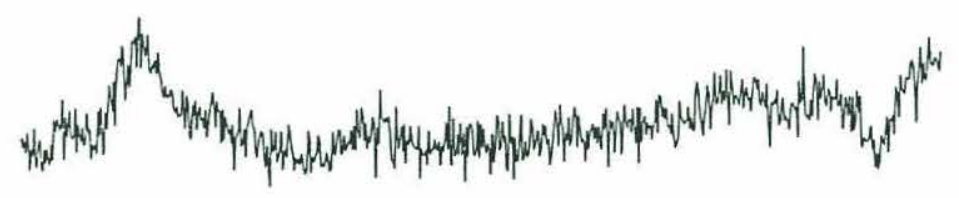

Fig. 5.12

$\begin{array}{llllll}5.40 & 5.30 & 5.20 & 5.10 & 5.00 & 4.90\end{array}$

${ }^{1}$ H NMR spectrum of three gel permeation chromatography fractions of pullulan (zero time): $\geq 10,000$ daltons (a), $\sim 5000$ daltons (b), $\leq 1200$ daltons (c). A small quantity of $\alpha(1,4)$ anomer (probably from traces of maltotriose) is visible in spectrum (c). 


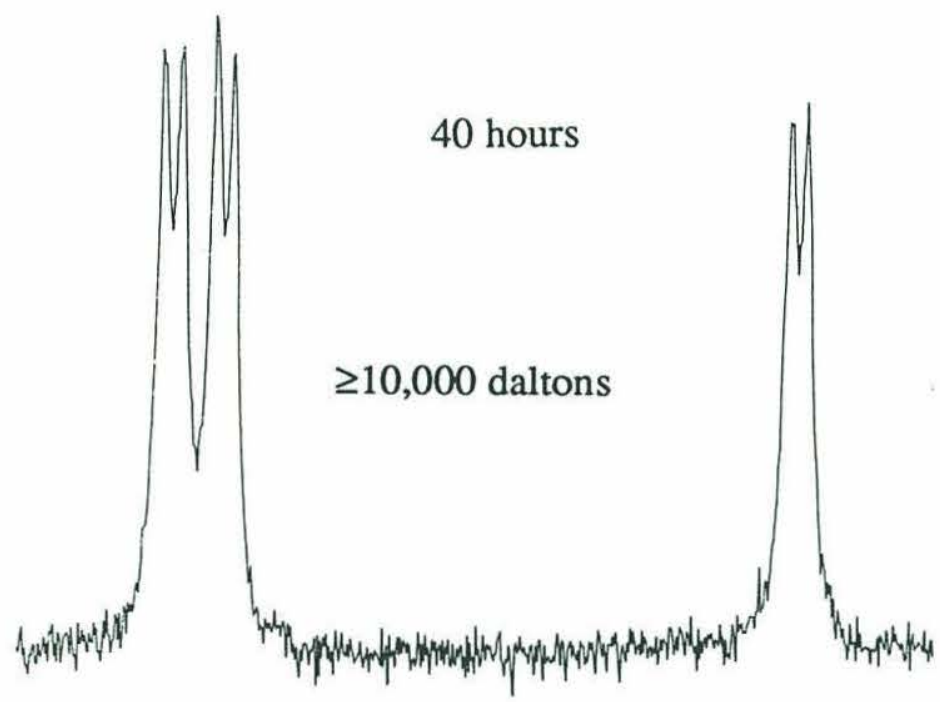

(a)

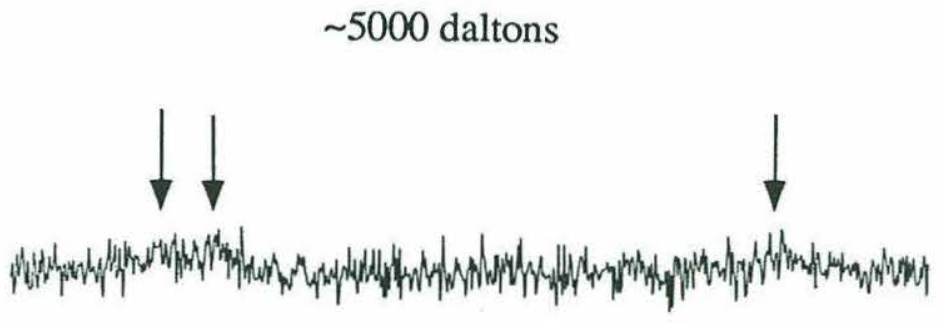

(b)

$$
\leq 1200 \text { daltons }
$$

(c)
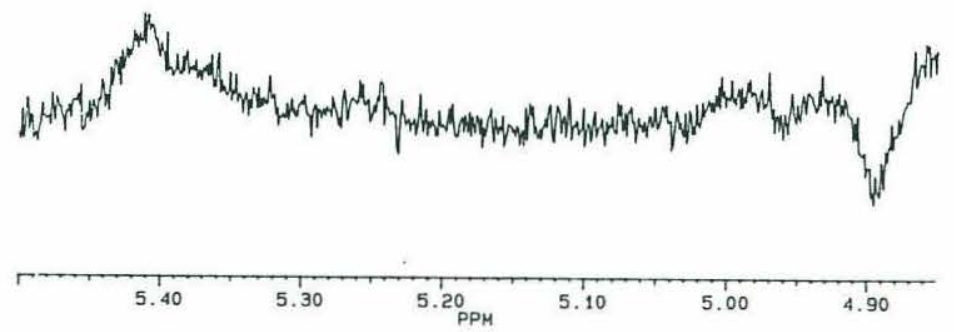

Fig. 5.13

${ }^{1} \mathrm{H}$ NMR spectrum of three gel permeation chromatography fractions of pullulan (40 hours): $\geq 10,000$ daltons (a), $\sim 5000$ daltons (b), $\leq 1200$ daltons (c). A small amount of pullulan is evident in the 5000 -dalton size class (b). 


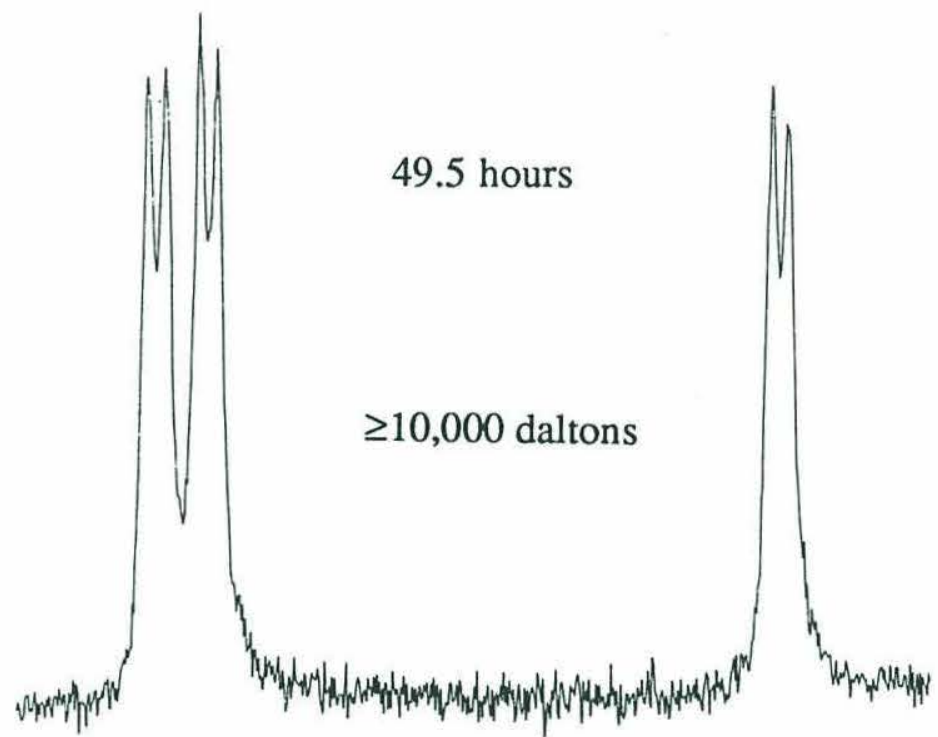

(a)

$\sim 5000$ daltons

(b)

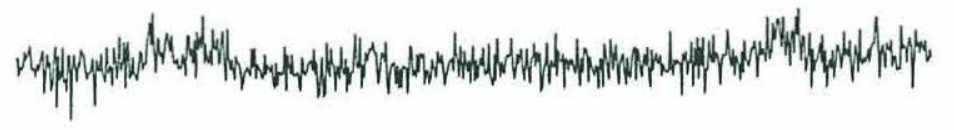

$\leq 1200$ daltons

(c)

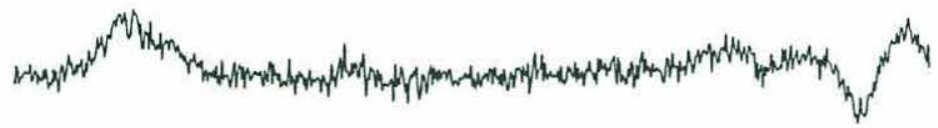

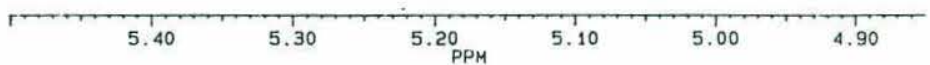

Fig. 5.14

${ }^{1} \mathrm{H}$ NMR spectrum of three gel permeation chromatography fractions of pullulan (49.5 hours): $\geq 10,000$ daltons (a), $\sim 5000$ daltons (b), $\leq 1200$ daltons (c). 


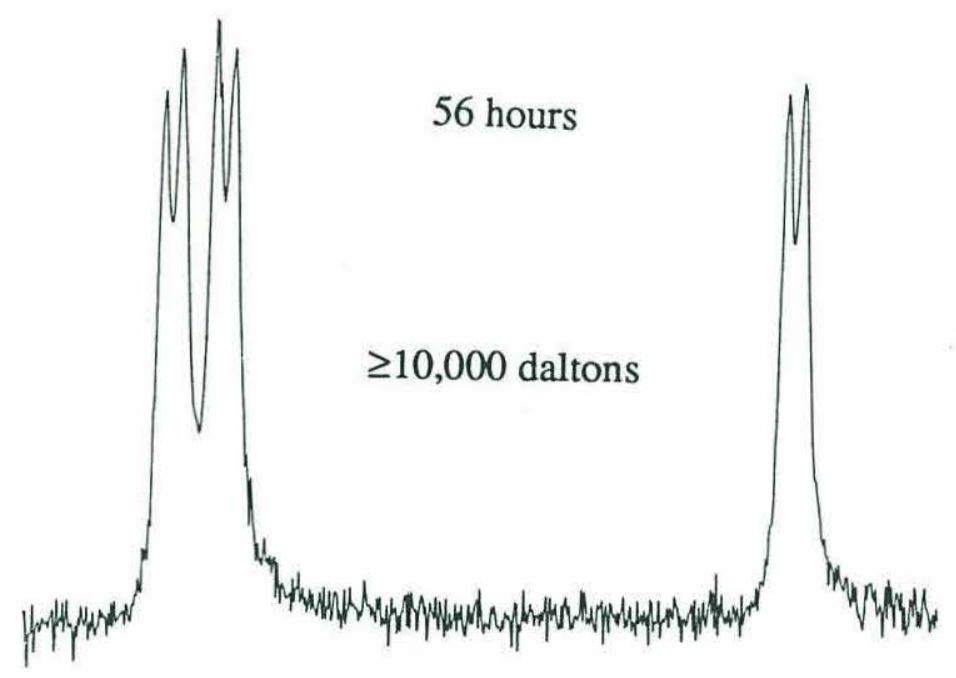

(a)

$\sim 5000$ daltons

(b)

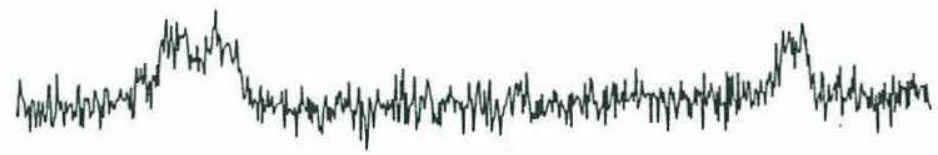

$$
\leq 1200 \text { daltons }
$$

(c)
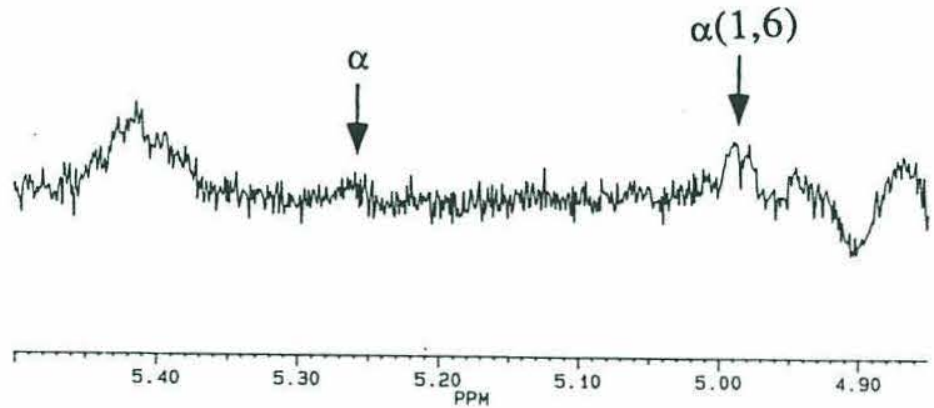

Fig. 5.15

${ }^{1} \mathrm{H}$ NMR spectrum of three gel permeation chromatography fractions of pullulan (56 hours): $\geq 10,000$ daltons (a), $~ 5000$ daltons (b), $\leq 1200$ daltons (c). Significant quantities of pullulan are in the 5000 dalton molecular weight range (b). A well-resolved $\alpha(1,6)$ resonance and a free $\alpha$-anomer are evident in the 1200 dalton molecular weight range (c). 


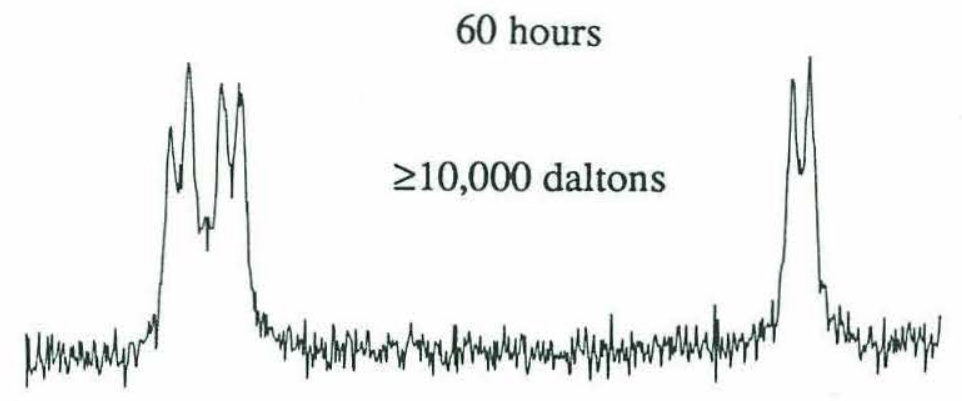

(a)

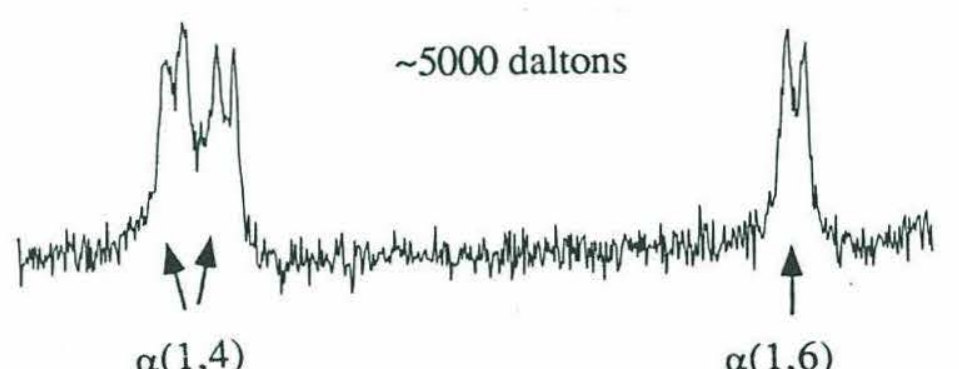

(b)

Fig. 5.16
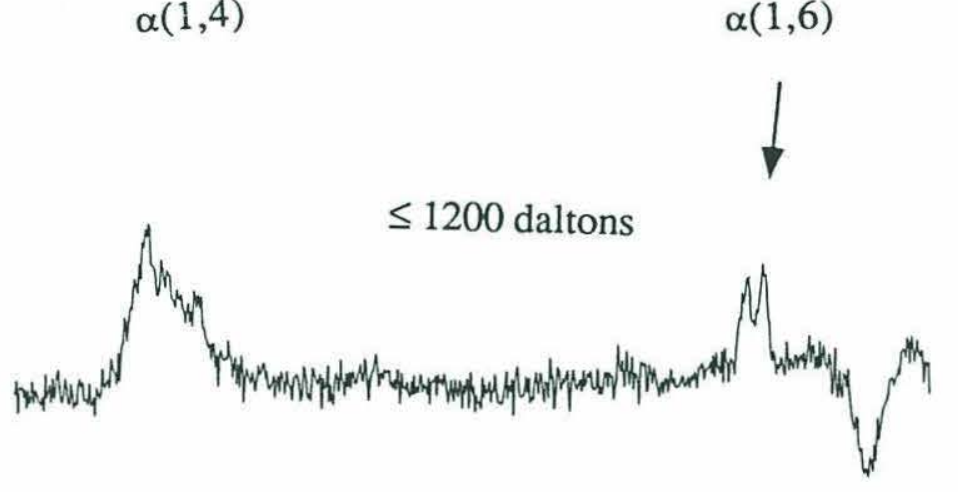

(c)

${ }^{1} \mathrm{H}$ NMR spectrum of three gel permeation chromatography fractions of pullulan (60 hours): $\geq 10,000$ daltons (a), $~ 5000$ daltons (b), $\leq 1200$ daltons (c). Quantity of pullulan in the $\geq 10,000$ daltons molecular weight range (a) is significantly reduced, and quantity of pullulan in the 5000 dalton molecular weight range (b) is significantly increased. Note that the J-couplings in (b) for both $\alpha(1,4)$ anomers and for the $\alpha(1,6)$ anomer are clearly resolved. J-coupling of $\alpha(1,6)$ anomer is resolved in the 1200 dalton molecular weight range (c), but the $\alpha(1,4)$ anomeric resonance is broad and shows no distinctive J-coupling. 
64 hours

$\geq 10,000$ daltons

(a)

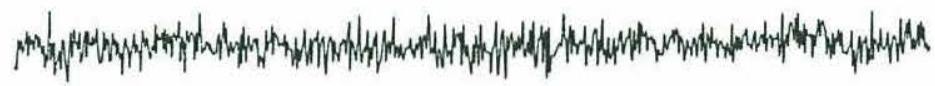

$\sim 5000$ daltons

(b)

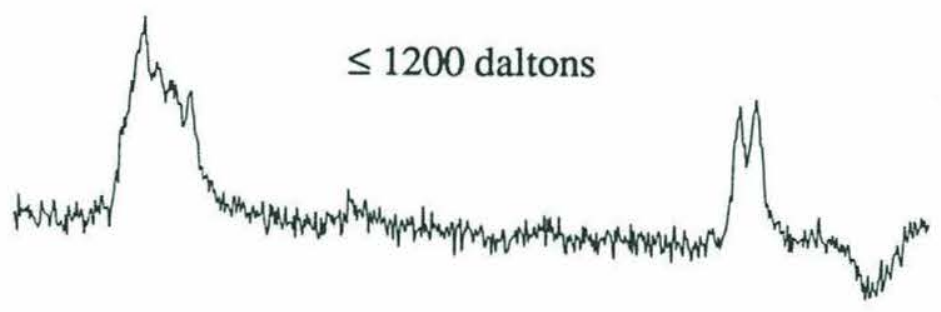

(c)

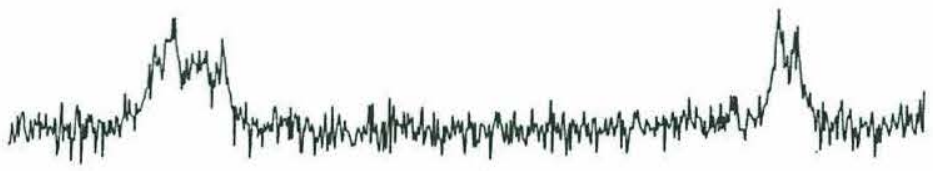

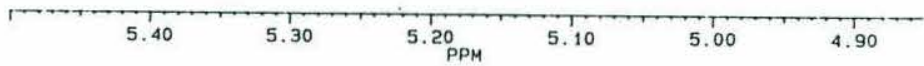

Fig. 5.17

${ }^{1} \mathrm{H}$ NMR spectrum of three gel permeation chromatography fractions of pullulan (64 hours): $\geq 10,000$ daltons (a), $~ 5000$ daltons (b), $\leq 1200$ daltons (c). No pullulan remains in the 10,000 dalton molecular weight range (a). The 5000 dalton molecular weight range (b) has less pullulan than for the previous time point, while the quantity of substrate in the 1200 molecular weight range (c) has increased. 
69 hours

$\geq 10,000$ daltons

(a)

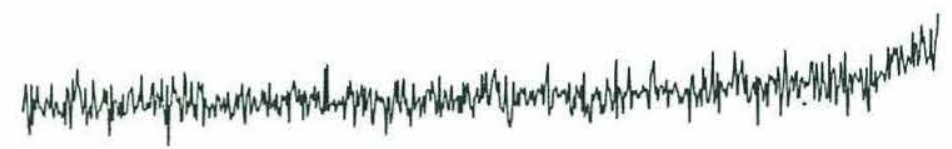

$\sim 5000$ daltons

(b)

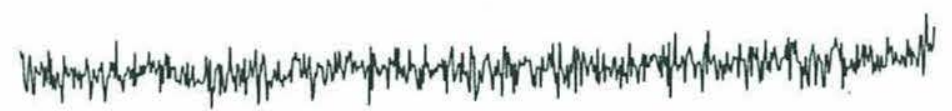

$\leq 1200$ daltons

(c)

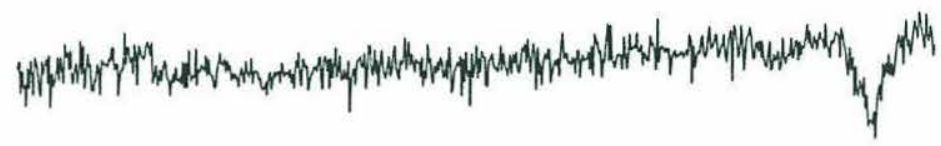

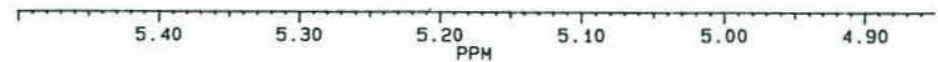

Fig. 5.18

${ }^{1} \mathrm{H}$ NMR spectrum of three gel permeation chromatography fractions of pullulan (69 hours): $\geq 10,000$ daltons (a), $\sim 5000$ daltons (b), $\leq 1200$ daltons (c). No pullulan in any molecular weight range. 


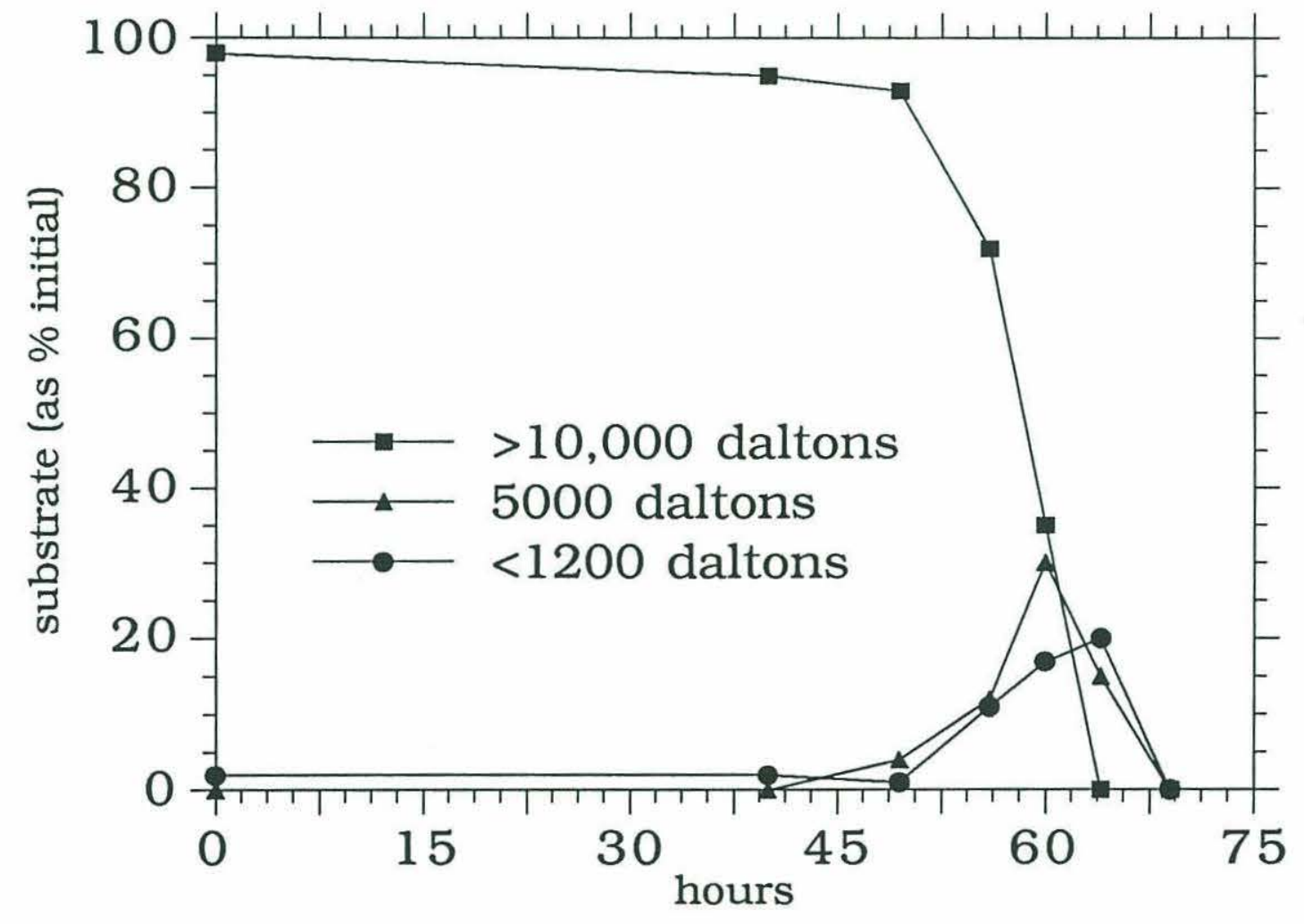

Fig. 5.19

Summary of changes in pullulan size classes during degradation. The plot shows changes in the areas of the anomeric resonances in the $\geq 10,000$ dalton, 5000 dalton, and $\leq 1200$ dalton GPC size classes as shown in NMR spectra (Figs. 5.12-5.18). 


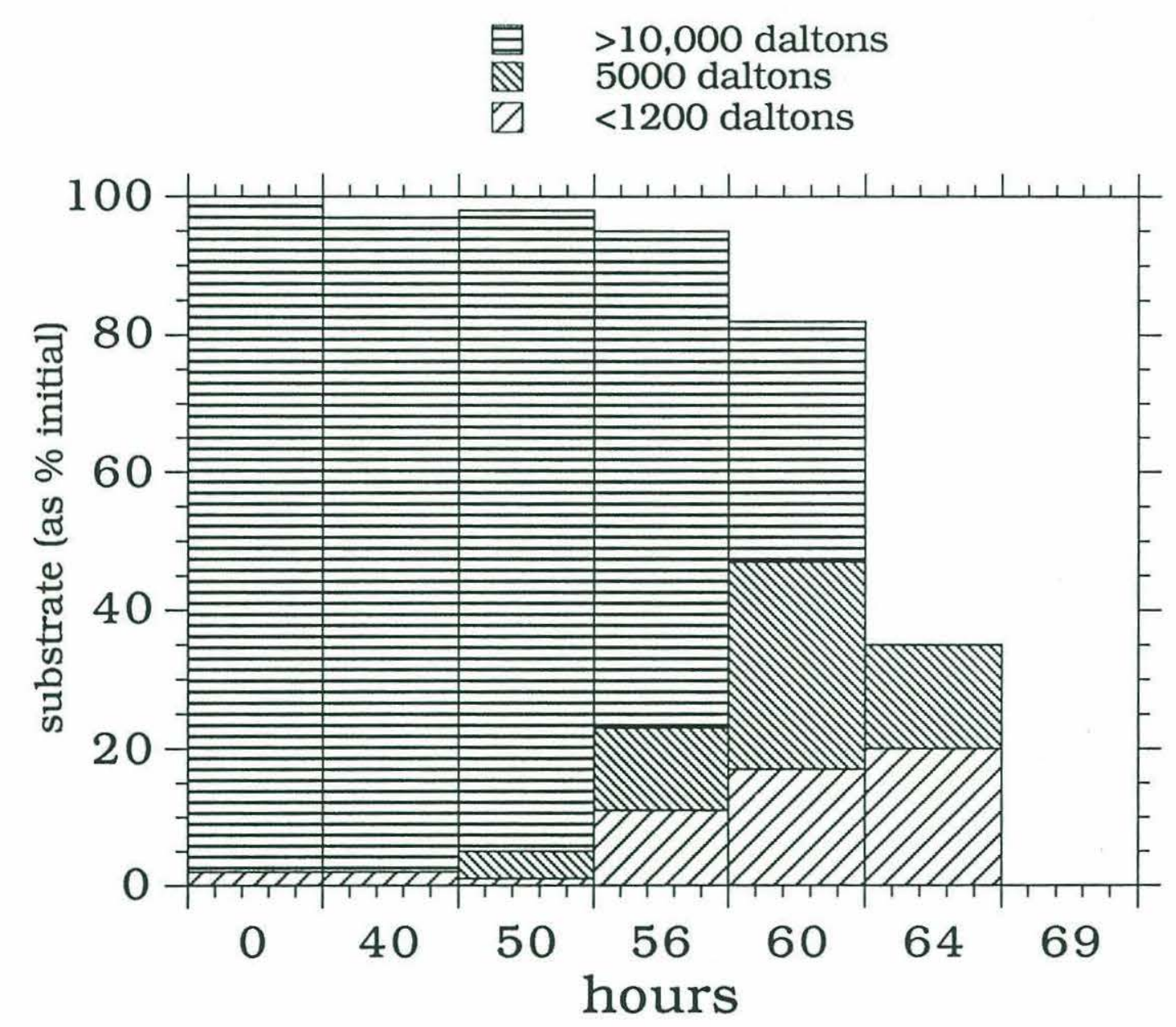

Fig. 5.20

Changes in distribution of substrate among $\geq 10,000$ dalton, 5000 dalton, and $\leq 1200$ dalton size classes during pullulan degradation. (Data replotted from Fig. 5.19.) 
pullulan was still in the medium. Rapid degradation of substrate commenced between 60 and 64 hours.

\section{Enzyme Specificity}

The changes in the anomeric resonances in the 1200 dalton fraction show that pullulan is preferentially hydrolyzed at the $\alpha(1,6)$ linkage. In Fig. 5.16c, for example, the anomer at $\delta=4.95$ is well-resolved, with J-coupling corresponding to that observed in Fig. 5.16a and b. The anomeric resonances in Fig. $5.16 \mathrm{c}$ centered around $\delta=5.4$ (corresponding to the $\alpha(1,4)$ linkages) have broadened and become less distinct. The broadening and loss of resolution among these resonances, in contrast to the clean anomeric resonance at $\delta=4.95$, shows that the $\alpha(1,6)$ linkage is being preferentially cleaved to produce oligosaccharides. In 200,000-dalton pullulan, and even in the pullulan hydrolyzed to the molecular weight range of 5000 daltons, there are essentially only two types of $\alpha(1,4)$ linkage environments (in NMR terms), corresponding to the linkages connecting the first and the second, and the second and third, units in the maltotriose sections of the polymer. Once oligosaccharides have been produced by hydrolysis of the $\alpha(1,6)$ linkage, the chemical shifts of the $\alpha(1,4)$ linkages are slightly shifted depending upon position in the oligosaccharide chain, hence the loss of resolution in this area of the spectrum. (A similar situation was observed in Figs. 3.8 and 3.9, where the presence of $\alpha(1,6)$ branches slightly changed the chemical shift of the $\alpha(1,4)$ resonances.) If both the $\alpha(1,4)$ and the $\alpha(1,6)$ linkages were being cleaved, the same type of broadening and loss of resolution would be expected for the $\alpha(1,6)$ resonance. In addition, the ratio of the areas represented by the $\alpha(1,4)$ and $\alpha(1,6)$ linkage in pullulan is 2:1 (two maltotriose linkages to one $\alpha(1,6)$ linkage joining successive maltotriose units). The ratio holds for the 5000 dalton fraction as well, but for the 1200 dalton fraction, the area of the $\alpha(1,6)$ linkage is clearly less than half of the area of the $\alpha(1,4)$ linkages. Acquisition of a high-resolution ${ }^{1} \mathrm{H}$ NMR spectrum $(12,800$ scans, acquisition time $\sim 27$ hours) of the 60 hour, 1200 dalton fraction (shown in Fig. 5.16c) showed that the ratio of the $\alpha(1,4)$ to $\alpha(1,6)$ to free $\alpha$ anomers was $4.0: 1.0: 0.3$, which is exactly the ratio expected for two maltotriose units linked by a single $a(1,6)$ linkage (Fig. 5.21). (The free $\beta$ anomer could not be resolved from the ringing and spinning sidebands upfield of the HOD resonance.)

Preferential cleavage of the $\alpha(1,6)$ suggests a further important point about the action of the enzymes hydrolyzing the pullulan: they must be endo-exoenzymes, capable of hydrolyzing linkages in the interior of the polysaccharide chain, and not exo-exoenzymes which can only hydrolyze the non-reducing terminal unit of a polysaccharide chain. 


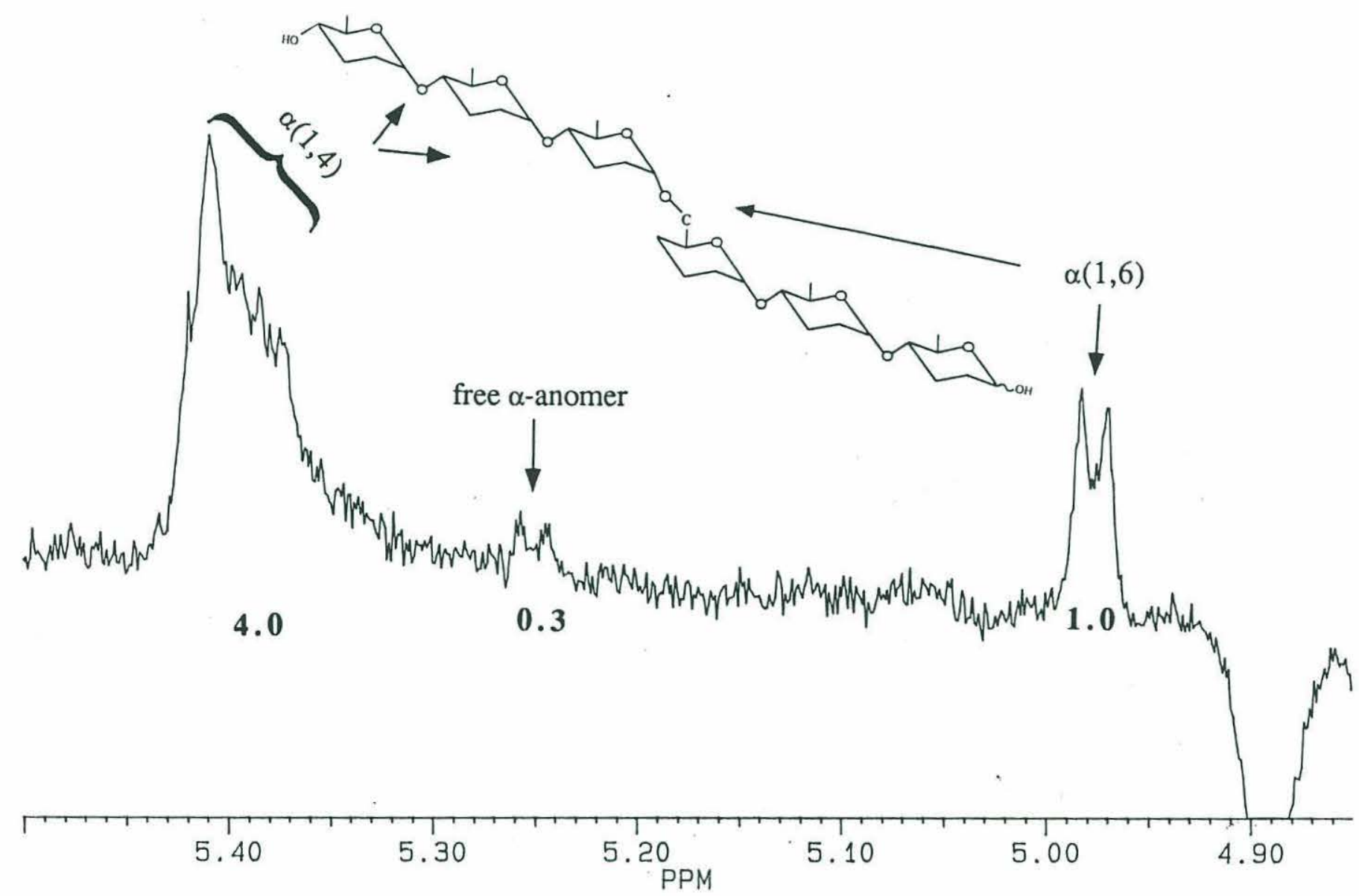

Fig. 5.21

High-resolution ${ }^{1} \mathrm{H}$ NMR spectrum of $\leq 1200$ dalton gel permeation fraction of pullulan (60 hours; same fraction as Fig. 5.16c). The ratio of the $\alpha(1,4)$ to $\alpha(1,6)$ to free $\alpha$ anomers exactly matches the ratio expected for two maltotriose units with an $\alpha(1,6)$ linkage $(4.0$ : 1.0: 0.3 ). 
Four different pullulanases and pullulan hydrolases, with different specificities, have been found in a range of bacteria. The enzymes in the pullulan cultures cannot be Type I or II pullulan hydrolases, since these enzymes attack $\alpha(1,4)$ linkages in pullulan, yielding panose or isopanose (Antranikian, 1992). Type I and II pullulanases, however, specifically cleave $\alpha(1,6)$ linkages. Type I pullulanase cleaves both $\alpha(1,6)$ branches in branched polysaccharides such as amylopectin, and $\alpha(1,6)$ linkages in pullulan. This endo-enzyme typically produces maltotriose and a series of $\alpha(1,6)$-linked maltotriose-oligosaccharides from pullulan. Type II pullulanase acts similarly to Type I pullulanase, but can hydrolyze $\alpha(1,4)$ linkages in addition to $\alpha(1,6)$ linkages (Koch and Antranikian, 1990). Hydrolysis of the $\alpha(1,6)$ linkage seems to be preferential, however, since Type II pullulanases convert pullulan into a series of $\alpha(1,6)$ maltotriose-oligosaccharides. Both pullulanases can only hydrolyze $\alpha(1,6)$ linkages which are in the viscinity of $\alpha(1,4)$ linkages (i.e., isomaltose and isomaltotriose are not hydrolyzed by pullulanase), which suggests that specific carbohydrate conformations are necessary for the enzyme-substrate complex (Antranikian, 1992).

The most active pullulan-degrading enzyme in the pullulan cultures must therefore be either Type I or Type II pullulanase. The evidence from the NMR spectra is consistant with either enzyme, since both hydrolyze pullulan to maltotriose and $\alpha(1,6)$-linked maltotriose oligosaccharides. Type I pullulanase, however, has been identified only in the aerobic bacteria Klebsiella pneumoniae and Bacillus acidopullulyticus (Klingeberg et al., 1990) and in an anaerobic thermophilic species of Fervidobacterium (Antranikian, 1992). Type II pullulanase, in contrast, has been found in a wide range of anaerobes. Because of their potential utility in industrial applications (saccharification of commercial polysaccharides), investigation of these enzymes has focused almost exclusively on thermophilic and extremly thermophilic bacteria, including thermophilic clostridia (Klingeberg et al., 1990). Few studies have focused on marine bacteria; a search of the literature revealed that the only marine organisms reported to produce pullulan were the hyperthermophilic marine archaebacteria Pyrococcus furiosus (Brown et al., 1990) and Pyrococcus woesi (Antranikian, 1992).

The anaerobic bacteria enriched from sulfate-reducing sediments therefore most likely produce Type II pullulanase, which is found in other anaerobes as well. A search of the literature has not revealed any other reports or investigations of pullulanase activity among mesophilic marine bacteria. In addition, most studies have been carried out on pure cultures of bacteria or on enzymes isolated from pure cultures. Pullulanase activity in growing cultures typically was determined by monitoring the quantity of reducing sugars in the medium. Specific extracellular pullulanase activity was measured by incubating the cell- 
free medium with pullulan, and monitoring production of glucose and oligosaccharides (DP2-DP6) using an HPLC column and an unspecified detection method (Klingeberg $e t$ al., 1990). In studies using only isolated enzymes, the hydrolysis of starting substrate was determined by measuring reducing sugars colorimetrically, while production of oligosaccharides was determined by HPLC or thin-layer chromatography (Koch and Antranikian, 1990) (Brown et al., 1990). This study therefore is the first report of pullulanase activity among marine mesophiles, as well as a first determination of 'intermediate' hydrolysis steps, since the degradation of pullulan to smaller polysaccharides, as well as oligosaccharides, was monitored.

\section{Implications for Early Diagenesis of Organic Matter in Marine Sediments}

Most organic matter is biosynthesized as high molecular weight cellular components such as proteins, lipid complexes, and polysaccharides. Research in organic geochemistry, however, has focused on the lower molecular weight components or on hydrolysis products (amino acids, monosaccharides) of the high molecular weight material. An important feature of marine organic matter, therefore, is largely unknown. Because little is known about the structure of biological macromolecules, we do not know which factors control the rates and processes by which organic matter is cycled in the water column and sediments, or why nominally labile components of organic matter (such as carbohydrates and proteins) are resistent to degradation beyond a given depth in recent sediments. Although this discrepancy between our knowledge of low and high molecular organic matter and the quantitative importance of these pools has long been recognized (Burdige, 1991; Henrichs, 1992; Lee, 1992), progress in characterizing the nature and dynamics of macromolecular organic matter has been slow. As previously discussed for Fig. 1.1, while there are estimates of input, burial, and upward flux terms, the cycling among the particulate macromolecule $\longrightarrow$ dissolved macromolecule $\longrightarrow$ dissolved monomer boxes is poorly understood and completely unquantified.

Microbiologists have also long been concerned with these transformations in the context of bacterial physiology and biochemistry, and have attempted to determine the mechanisms by which bacteria enzymatically hydrolyze substrates which are too large to directly ingest (Pollock, 1962, for example). In studies of marine systems, a range of fluorescent substrate analogs such as methylumbelliferyl (MUF) glycosides have been used to try to characterize the nature and activities of bacterial exoenzymes in seawater and sediments. Because natural substrate concentrations are unknown, rate comparisons are 
only relative. Even with this limitation, however, MUF substrates have provided useful information about varying sensitivities to different MUF-monosaccharides, and apparent depth-related and seasonal changes in enzyme activity (Somville, 1984; King, 1986; Meyer-Reil, 1987). The MUF substrates, however, are not necessarily adequate representatives of polysaccharide hydrolysis. Among rumen bacteria, for example, there are numerous examples of organisms which grow efficiently on oligosaccharides, although they do not grow on related polysaccharides because they cannot produce the exoenzymes necessary to convert the polysaccharides to oligosaccharides (Russell, 1985; Cotta, 1992; Malburg et al., 1992). Bacteria which are capable of hydrolyzing and utilizing oligosaccharides (which the MUF substrates are supposed to model) do not necessarily have the enzymatic capability to hydrolyze polysaccharides to oligosaccharides. In addition, the MUF substrates cannot be used determine the activity of certain enzymes such as pullulanase. MUF substrates do not conform to the structural specificity of pullulanase, which requires $\alpha(1,4)$ linkages on either side of the target $\alpha(1,6)$ linkage (White and Kennedy, 1988).

The results of the polysaccharide degradation experiments help begin to bridge these large gaps in both geochemical and microbiological studies of early diagenesis. Chapters 4 and 5 present an alternative method for studying the early diagenesis of organic matter, through which the time course of alterations in substrate structure can be specifically determined, and the effects of variations in substrate structural features can be directly compared. The degradation of pullulan is apparently the first molecular-level evidence in marine systems of the bacterial extracellular hydrolysis of macromolecular to low molecular weight organic matter. The closely-spaced sampling intervals, combined with size- and structural information obtained with gel permeation chromatography and NMR spectroscopy permitted precise determination of the degradation mechanism, and by implication, of the specific enzyme responsible for substrate hydrolysis.

While the culture system used in these experiments does not precisely replicate natural sediment systems (substrate levels in the cultures, for example, are much higher than found in natural sediments), it shares many features of natural sediment communities which might develop after the 'crash' of a phytoplankton bloom, for example. In addition, degradation of the substrate which was added at a low level (F2, at a concentration of 5.1 $\mu \mathrm{g} / \mathrm{mL}$ ) occurred on the same time scale as the degradation of the substrates added at much higher level, which suggests that substrate concentrations did not significantly affect culture development.

The behavior of the culture system was quantitative, consistent, and reproducible through multiple replicates over several years, which supports the assumption that these 
cultures are reasonable models of natural bacterial consortia. While absolute rates of organic matter degradation cannot be determined, the processes and relative rates of degradation are significant. An important conclusion is that gross size of glucose polysaccharides and teichoic acid-type polysaccharides is not a significant determinant of degradation rate. The transition from dissolved polysaccharide to transportable oligosaccharide, although it could be observed with high-resolution sampling, was not the slow step in degradation, since lower molecular weight carbohydrate intermediates accumulated, and the polysaccharide was degraded on the same timescale as its component units of maltotriose (Chapter 4). In certain cases, therefore, the dissolved polymer $\rightarrow$ dissolved monomer transition is not the slow step in degradation of organic matter. High molecular weight does not necessarily correlate with slower degradation rates. This result is directly contrary to the widely-held (but not well tested) belief that the enzymatic hydrolysis of macromolecules by bacteria determines organic matter degradation rate (Meyer-Reil, 1987; Hoppe, 1991, for example; Meyer-Reil, 1991).

In this regard, the relatively slow degradation of GLA is even more surprising: the smallest substrate used in the culture series persisted for the longest time in the culture. Even once degradation of GLA had begun, 120 hours elapsed before the substrate was completely consumed. For F3, laminarin, and pullulan, the interval between onset of degradation and complete substrate consumption was on the order of 10-15 hours. The order-of-magnitude difference in rate of substrate degradation, on top of the long degradation lag, may suggest that GLA degrading bacteria are not only rare in the initial inoculum, they also have a low doubling rate. Substrate structural features clearly can have a significant effect on degradation rates, even at molecular weights below the direct transport size limit. 


\section{References}

Antranikian, G. (1992). Microbial degradation of starch. Microbial Degradation of Natural Products. New York, VCH. 27-56.

Brown, S. H., H. R. Costantino and R. M. Kelly (1990). Characterization of amylolytic enzyme activities associated with the hyperthermophilic archaebacterium Pyrococcus furiosus. Appl. Environ. Microbiol. 56(7): 1985-1991.

Burdige, D. J. (1991). The kinetics of organic matter mineralization in anoxic marine sediments. J. Mar. Res. 49: 727-761.

Burdige, D. J. and C. S. Martens (1988). Biogeochemical cycling in an organic-rich coastal marine basin: 10 . The role of amino acids in sedimentary carbon and nitrogen cycling. Geochim. Cosmochim. Acta 52: 1571-1584.

Cotta, M. A. (1992). Interaction of ruminal bacteria in the production and utilization of maltooligosaccharides from starch. Appl. Env. Microbiol. 58(1): 48-54.

Dehorty, B. A. (1967). Rates of isolated hemicellulose degradation and utilization by pure cultures of cellulolytic rumen bacteria. J. Bacteriol. 89: 1515-1520.

Gottschalk, G. (1986). Bacterial Metabolism. Berlin, Springer-Verlag.

Henrichs, S. M. (1992). Early diagenesis of organic matter in marine sediments: progress and perplexity. Mar. Chem. 39: 119-149.

Hespell, R. B., R. Wolf and R. J. Bothast (1987). Fermentation of xylans by Butyrivibrio fibrisolvens and other ruminal bacteria. Appl. Environ. Microbiol. 53(12): 2849-2853.

Hoppe, H.-G. (1991). Microbial extracellular enzyme activity: a new key parameter in aquatic ecology. Microbial Enzymes in Aquatic Environments. Berlin, Springer. 60-83.

Hudson, J. A., H. W. Morgan and R. M. Daniel (1990). Cellulolytic properties of an extremely thermophilic anaerobe. Appl. Microbiol. Biotechnol. 33: 687-691.

Hungate, R. E. (1966). The Rumen and Its Microbes. New York, Academic Press.

Hungate, R. E. (1969). A roll tube method for cultivation of strict anaerobes. Methods in Microbiology. New York, Academic Press.

King, G. M. (1986). Characterization of $\beta$-glucosidase activity in intertidal marine sediments. Appl. Environ. Microbiol. 51(2): 373-380.

Klingeberg, M., H. Hippe and G. Antranikian (1990). Production of novel pullulanases at high concentrations by two newly isolated thermophilic clostridia. FEMS Microb. Lett. 69: $145-152$.

Koch, R. and G. Antranikian (1990). Action of amylolytic and pullulytic enzymes from various anaerobic thermophiles on linear and branched glucose polymers. Starch 42: 397403. 
Koch, R., P. Zablowski, A. Spreinat and G. Antianikian (1990). Extremely thermostable amylolytic enzyme from the archaebacterium Pyrococcus furiosus. FEMS Microbiol. Lett. 71: 21-26.

Lee, C. (1992). Controls on organic carbon preservation: the use of stratified water bodies to compare intrinsic rates of decomposition in oxic and anoxic systems. Geochim.

Cosmochim. Acta 56: 3323-3335.

Malburg, L. M., J. M. Tamblyn Lee and C. W. Forsberg (1992). Degradation of cellulose and hemicellulose by rumen microorganisms. Microbial Degradation of Natural Products. New York, VCH. 127-159.

Meyer-Reil, L.-A. (1987). Seasonal and spatial distribution of extracellular enzymatic activities and microbial incorporation of dissolved organic substrates in marine sediments. Appl. Environ. Microbiol. 53(8): 1748-1755.

Meyer-Reil, L.-A. (1991). Ecological aspects of enzymatic activity in marine sediments. Microbial Enzymes in Aquatic Environments. Berlin, Springer. 84-95.

Miura, A., M. Horiguchi, D. Ogimoto and T. Matsumoto (1983). Nutritional interdependence among rumen bacteria during cellulose digestion in vitro. Appl. Environ. Microb. 45: 726-729.

Pollock, M. R. (1962). Exoenzymes. The Bacteria. New York, Academic Press. 121-178.

Robson, L. M. and G. H. Chambliss (1989). Cellulases of bacterial origin. Enzyme Microb. Technol. 11: 626-644.

Russell, J. B. (1985). Fermentation of cellodextrins by cellulolytic and noncellulolytic rumen bacteria. Appl. Environ. Microbiol. 49(3): 572-576.

Somville, M. (1984). Measurement and study of substrate specificity of exoglucosidase activity in eutrophic water. Appl. Environ. Microbiol. 48(6): 1181-1185.

Weimer, P. J. and J. G. Zeikus (1977). Fermentation of cellulose and cellobiose by Clostridium thermocellum in the absence and presence of Methanobacterium thermoautotrophicum. Appl. Environ. Microbiol. 33(2): 289-297.

White, C. A. and J. F. Kennedy (1988). The carbohydrate-directed enzymes. Carbohydrate Chemistry. Oxford, Clarendon Press. 343-377.

Williams, A. G. and S. E. Withers (1982). The production of plant cell wall polysaccharide-degrading enzymes by hemicellulolytic rumen bacterial isolates grown on a range of carbohydrate substrates. J. Appl. Bact. 52: 377-387.

Wood, W. A. (1961). Fermentation of Carbohydrates and Related Compounds. The Bacteria. New York, Academic Press. 59-149. 
210 


\section{Chapter Six: Summary}

Most organic matter is synthesized as high molecular weight cellular components such as proteins, lipid complexes, and polysaccharides. In marine systems, the majority of this organic matter is rapidly remineralized back to $\mathrm{CO}_{2}$; only a small fraction of total primary productivity is ultimately buried in sediments as recalcitrant organic matter. Research in organic geochemistry, however, has focused primarily on low molecular weight components or hydrolysis products (amino acids, small lipids, monosaccharides) of macromolecules. Our inability to structurally characterize intact macromolecules has hampered efforts to determine rates and mechanisms of organic matter degradation and preservation in marine sediments. The factors governing transformations of macromolecular organic matter in marine sediments are important 'unknowns' in studies of early diagenesis.

In anoxic sediments, which account for more than $90 \%$ of the annual burial of organic carbon (Henrichs and Reeburgh, 1987), bacteria are the only organisms capable of degrading macromolecular organic matter to $\mathrm{CO}_{2}$ and simple substrates. While the bacterial degradation of simple substrates is well understood, little is known about the rates and mechanisms by which consortia of bacteria degrade macromolecules. Studies of pure cultures of bacteria have demonstrated that many bacteria produce extracellular enzymes to hydrolyze large substrates to smaller pieces which they can ingest. These extracellular enzymes selectively cleave structures with specific conformations and chemical linkages (Rogers, 1961; Priest, 1992). Substrate structure therefore must play a role in bacterial degradation of organic matter.

The underlying theme of this thesis is the connection between chemical structure and reactivity: what structural factors determine the rate and pathways by which bacteria degrade macromolecular carbohydrates in anoxic sediments? The impetus for the focus on carbohydrates was the observation that monosaccharides such as glucose are readily metabolized by most organisms, but many high molecular weight polymers of simple sugars (such as cellulose) can be degraded only by a select range of bacteria, protozoa, and fungi. This range in reactivity exists even though individual monosaccharides are structurally quite similar. High molecular weight carbohydrates are classic biological polymers, which differ primarily in the number of repeating units, vary somewhat in monomer composition, and have a well-defined range of linking and branching possibilites.

A combination of chemical and microbiological techniques were used to investigate the influence of size, linkage position, linkage orientation, and monomer composition on 
the rate and pathways by which consortia of anaerobic bacteria degrade carbohydrates. Hungate anaerobic techniques (Hungate, 1969) were used to enrich replicate cultures of bacteria from sulfate-reducing sediments on structurally well-characterized substrates. The substrates, intermediates, and degradation products were quantified and characterized using a variety of chromatographic and spectroscopic techniques. The basis for nuclear magnetic resonance (NMR) spectroscopic determinations of carbohydrate structure was laid out in Chapter 2. A variety of 1- and 2-D NMR experiments was used to investigate the structure of several carbohydrate standards, and several corrections and additions to published ${ }^{1} \mathrm{H}$ and $13 \mathrm{C}$-chemical shifts of oligo- and polysaccharides were made.

In Chapter 3, NMR spectroscopy was applied to the characterization of waterextractable plankton carbohydrates. The structures of three specific polysaccharides were determined. Dunaliella tertiolecta contained large quantities of an amylose-like $(\alpha(1,4)$ linked) polysaccharide; extracts of Synechococcus WH7335 contained smaller quantities of an amylopectin-like $(\alpha(1,4)$ with $\alpha(1,6)$ branches) glucose polysaccharide. Both of these polysaccharides probably are accumulated as energy reserves. The cold aqueous extract of Synechococcus WH7335 consisted principally of a glycerol-polysaccharide, which was tentatively identified as a teichoic acid. Teichoic acids of similar structure are found in the cell walls of gram-positive bacteria; this is the first suggestion of their occurrence in cyanobacteria.

The value of NMR spectroscopy in structural determinations was particularly evident in the characterization of the Synechococcus teichoic acid. While independent determinations of monomer composition and linkage position were necessary, the 1- and 2-D NMR spectra were critical in constraining the types of structures which were consistent with the compositional data. Discovery of the teichoic acid-type polysaccharide also brings up a truism about investigations of the chemical composition of organisms: you find what you are looking for. Techniques used in previous studies of the glycolipid composition of cyanobacteria would not have extracted teichoic acid of this type, and methods used to characterize the carbohydrate composition of cyanobacteria would have destroyed this labile structure.

The oligosaccharide degradation experiments in Chapter 4 demonstrate that the culture system produced quantitative and reproducible results. The sequence of transformations observed in the cultures were consistent with the sequential substrate degradation expected in a consortia of anaerobic bacteria. The results of the di- and oligosaccharide experiments demonstrate that even mixed cultures of bacteria differentiate between closely-related structures. The slow degradation of GLA revealed that chemical structure is important, even within the size range of substrate which bacteria can transport 
without extracellular hydrolysis. The time-lag in GLA degradation suggests that most bacteria simply cannot degrade this particular structure, in spite its small size and the fact that neither its component pieces nor its linkage position or orientation were unusual. The lag in substrate degradation most likely reflected the time required for a very small percentage of the bacteria in the initial inoculum to double sufficiently to completely degrade the substrate. The decrease in degradation lag in a transfer culture supports the hypothesis that initial bacterial numbers were a cause of slow substrate degradation.

The development of the GLA cultures ( $\mathrm{CO}_{2}$ evolution, etc.) indicated that GLA is metabolized to standard products (acetate, lactate, $\mathrm{CO}_{2}$, etc.). This further suggests that internal transport or hydrolysis of the hexamer-pentamer disaccharide, not transformation of a fermentation product, was the slow step. Since many of the biochemical processes in carbohydrate metabolism involve phosphorylation of the \#1 or \#6 carbon, a disaccharide which lacks one \#6 carbon may not fit a standard enzymatic template. Because little information about the structure of marine carbohydrates is available, it is impossible to tell whether hexamer-pentamer carbohydrate combinations are common in marine systems.

The polysaccharide degradation experiments in Chapter 5 demonstrate that the hydrolysis of soluble macromolecules to ingestible substrates is not necessarily the slow step in polymer degradation. Bacterial degradation of pullulan and laminarin, as well as the branched glucan and the teichoic acid-type structure from Synechocccus WH7335, was rapid. An important conclusion from the degradation experiments is that high molecular weight does not always correlate with slow degradation rates. Differences in chemical structure can significantly affect degradation rates, but structure is not defined solely by gross size. This result directly contradicts the assumption that enzymatic hydrolysis of macromolecules is the rate-limiting step in organic matter degradation (Meyer-Reil, 1987; Hoppe, 1991; Meyer-Reil, 1991). The lack of a rate difference implies that enzyme induction and production of exoenzymes must occur very quickly. Rate differences in carbohydrate degradation (i.e., degradation of GLA compared to pullulan) occur on time scales consistent with the doubling time of bacteria, not with the production of enzymes.

The time course of the transformation of 200,000-dalton pullulan to $~ 5000$ dalton polysaccharides and to oligosaccharides was resolved using gel-permeation chromatography and NMR spectroscopy. This study provided the first molecular-level evidence in marine systems of the extracellular hydrolysis of a macromolecule to ingestible substrates. NMR spectra revealed that the pullulan was most likely hydrolyzed by a Type II pullulanase, an endo-acting extracellular enzyme which preferentially hydrolyzes $\alpha(1,6)$ linkages. The hydrolysis of pullulan was specific through all substrate size classes. Even when substrate hydrolysis was well advanced, there was a well-defined linkage pattern 
evident in the remaining substrate. The uniformity of linkage patterns suggests that pullulanase, not a mixture of pullulanase and amylolytic enzymes, was responsible for hydrolysis through all size classes of substrate.

This is the first report of pullulanase activity among mesophilic marine bacteria. The activity of pullulanase would not have been measured by previous studies of the enzymatic activity of bacteria in seawater or sediments (Somville, 1984; King, 1986; Meyer-Reil, 1987; Hoppe, 1991, for example), because the fluorescent substrate analogs used in those studies do not conform to the structural specificity of pullulanase. Type II pullulanase cleaves only $\alpha(1,6)$ linkages which have at least two $\alpha(1,4)$-linked monomers on either side (White and Kennedy, 1988).

The demostration of this activity, and the rapid overall degradation of pullulan, raise a question about the types of polysaccharides which may exist in marine environments. Results of the oligosaccharide degradation experiments suggest that a bacterial population in sediments may to some extent be 'primed' to degrade particular structures. Glucose homodisaccharides were in general degraded slightly faster than heterodisaccharides. The difference in time required for complete substrate degradation (on the order of 20-40 hours) suggests that induction of a specific enzyme was probably not the cause of slower degradation of heterodisacchaides, since enzyme induction typically occurs on a time scale of minutes (Gottschalk, 1986). Bacteria capable of degrading glucose homodisaccharides may either have been more numerous in the initial inoculum, or may have doubled more quickly, than the bacteria which degraded the heterodisaccharides. In the case of GLA, the ability to degrade the hexamer-pentamer disaccharide was probably very rare, since the degradation lag was more than twice as long as for any other substrate. Even after the onset of degradation, substrate degradation was an order of magnitude slower (120 hours for complete consumption) than observed for any other substrate. The rapid degradation of pullulan, on the other hand, implies that bacteria which can degrade this structure are either very numerous or double quickly. This in turn implies that its specific combination of $\alpha(1,4)$ and $\alpha(1,6)$ linkages may be common in marine systems. Further data on the linkage pattern of glucose polysaccharides produced in marine systems would be necessary to test this hypothesis. The rapid degradation of the polysaccharides also implies that the quantity of dissolved carbohydrates measured in seawater may not accurately reflect the importance of high molecular weight carbohydrates to bacteria, since high turnover rate may keep the standing stock of substrate very low.

The results of the degradation experiments demonstrate that neither anomeric linkage nor linkage position per se affect degradation of disaccharides. Glucose homodisaccharides of differing linkage position and orientation were degraded on the same 
timescale. Molecular weight also did not prove to be the slow step in degradation, since pullulan and maltotriose were degraded on the same time scale, in spite of the 400-fold difference in their molecular weights. The slow degradation of GLA, however, suggests that particular combinations of monosaccharides might be difficult or impossible for most bacteria to degrade. Although the difference in degradation rate between GLA and the other substrates was a matter of days, which is very little time on a sedimentary timescale, only a very small number of carbohydrate structures were used in the degradation experiments. Experiments with a wider variety of carbohydrates might reveal other structures which are resistant to degradation by bacteria. The small fraction of marine carbohydrates which is ultimately buried and preserved in sediments may in fact be distinguished by unusual structural features.

The relatively rapid degradation of the other two heterodisaccharide combinations (galactose-glucose, galactose-mannose) used in the culture experiments shows that not all combinations of monosaccharides are biochemical 'problems'. The fact that three of the eight most common neutral monosaccharides found in marine environments are pentamers, however, suggests that pentamer-hexamer combinations can probably be found among marine carbohydrates; all of the arabinose, rhamnose, and xylose found in organisms, particles, and sediments is not likely to occur solely in homopolysaccharides. In addition, two of five common hexameric monosaccharides are deoxy sugars, which have a methyl group at the \#6 carbon. None of the substrates used in the cultures included either fucose or rhamnose, but it is possible that deoxy sugars in combination with other monosaccharides in a polymer might also present biochemical problems. Addition of a phosphate group at the \#6 carbon of a monosaccharide, in fact, is dependent upon the presence of a free hydroxyl group at that position. Further investigations with different heterodisaccharides or heteropolysaccharide substrates could highlight other carbohydrate structures which are difficult for bacteria to hydrolyze or metabolize.

Phase transition, packaging, and reactions with other types of sedimentary organic matter are additional factors which may influence carbohydrate degradation. The substrates used in the culture series were all soluble, but in marine systems, deposition of particles at the sediment-water interface brings particulate carbohydrates to the sediments. The particulate macromolecule $\rightarrow$ dissolved polymer transition of Fig. 1.1 (D) was not investigated at all in this study, although it may be a rate-limiting factor in degradation of macromolecules. One intriguing idea for further work would be to use a relatively insoluble substrate in a further series of cultures (cellulose is an obvious example), and to monitor the transitions from insoluble carbohydrate $\rightarrow$ soluble polymers $\rightarrow$ soluble oligomers $\rightarrow \mathrm{CO}_{2}$. The only necessary addition to the techniques used in the polysaccharide 
degradation experiments would be a precise measure of remaining insoluble substrate, which could probably be quantified colorimetrically. Colorimetric determinations would be quite accurate in this case, since the monomer composition of the substrate is known.

Packaging may well be important, because the relative resistance of a soluble substrate such GLA to degradation may be significantly magnified if it were part of a cell wall polysaccharide, for example, sandwiched by murein layers or a thick coating of chitin.

Reactivity of carbohydrates with sedimentary organic matter has been investigated in a number of studies. As mentioned in Chapter 1, Klok et al. (1984) concluded that although only $4 \%$ of the total organic matter released during extraction procedures was directly identifiable as carbohydrates, $22 \%$ of sedimentary organic matter was actually derived from carbohydrates. The fact that pyrolysis was necessary to release these carbohydrate components from sediments implies that that they were very tightly bound in the sediment matrix. Moers et al. (1988) found even at ambient tempertures, glucose reacted with $\mathrm{H}_{2} \mathrm{~S}$ to form a variety of products. They suggested that formation of reactive thio-glucosides may lead to formation of other organic compounds which are resistent to biological or chemical degradation. Any 'inherent' resistance of an oligo- or polysaccharide to biological degradation may be further magnified by these types of processes.

\section{Suggestions for Further Work}

The work presented in this thesis opens a number of avenues for further research. The culture system could be used to study degradation of other types of carbohydrates, or other classes of organic matter such as proteins, glycoproteins, or lipopolysaccharides. The substrates used in the oligo- and polysaccharide cultures represent only a small percentage of the range of carbohydrate structures. Hexamer-pentamer carbohydrates or heteropolysaccharides might yield interesting results in a new series of cultures. As mentioned previously, the particulate $\longrightarrow$ dissolved macromolecule transition could be investigated using insoluble (or less soluble) carbohydrate substrates. From a microbiological perspective, isolation of select bacteria, such as the GLA-degrading bacteria or the mesophiles which produce Type II pullulanase, would be interesting. The GLA-degrading bacteria may have unique biochemical features which permit them to degrade unusual heterodisaccharides.

Since the culture system has been exhaustively tested in the laboratory, an extension to field studies is also important. Isotopically labeled substrates (or substrates with fluorescent tags) would probably be needed in order to do trace-level experiments. Labels would also simplify the problems associated with extracting a substrate from a complex 
matrix.

Extensive methods development is required to rigorously test the hypothesis that carbohydrates which are preserved in sediments differ in structure from their more labile counterparts which are degraded. The survey of plankton carbohydrates presented in Chapter 2 demonstrated that purification of specific carbohydrates from a complex biological matrix can be difficult; purification of intact polymeric carbohydrates from a sediment matrix will be even more of a challenge.

An indirect method of determining the structure of sedimentary carbohydrates would be to begin with the hypothesis that carbohydrates preserved at depth in the sediments differ in structure from labile carbohydrates in surface sediments. A core collected from a site with consistent organic matter sources (to minimize effects due to source changes) could be used to compare carbohydrates in surface and buried sediments. (Conventional extraction techniques could be used to determine total carbohydrates and the depth in the core at which carbohydrate degradation appears to level off.) A sequence of extraction techniques could be used in a first attempt to characterize the types of matrices in which the carbohydrate are bound at differen depths in the core. This would be an extension of the type of study carried out by Klok et al. (1984), but a range of techniques used in glycobiology (lectin affinity chromatography, selected enzymes which have only recently become commercially available, etc.) could be applied as well. The 'harshness' of the extraction method required to extract a particular fraction of carbohydrates should be related to the type of matrix in which the carbohydrate is found. With some of the milder extraction techniques, structural characterizations of the carbohydrates could also be made.

The phytoplankton survey in Chapter 3 is a first attempt at characterizing the macromolecular structure of plankton carbohydrates; much more could be done. A high priority in studies of marine carbohydrates should be development of methods to measure and characterize small quantities of intact carbohydrates. NMR spectroscopy is particularly promising in this respect, since higher sensitivity and better resolution can be achieved by using inverse techniques and higher-field magnets. Two analytical challenges stand out: (1) development of more effective extraction techniques which can recover intact carbohydrates from a variety of matrices; (2) development of desalting techniques which can be applied to the lower molecular weight ( $<1000$ dalton) size class of dissolved organic matter. Relative to their abundance in marine organic matter, very little work has been done to characterize marine carbohydrates. Development and application of new analytical techniques will be a key to more complete structural determination of marine carbohydrates. 


\section{References}

Gottschalk, G. (1986). Bacterial Metabolism. Berlin, Springer-Verlag.

Henrichs, S. M. and W. S. Reeburgh (1987). Anaerobic mineralization of marine sediment organic matter: rates and the role of anaerobic processes in the oceanic carbon economy. Geomicrobiol. J. 5(3/4): 191-237.

Hoppe, H.-G. (1991). Microbial extracellular enzyme activity: a new key parameter in aquatic ecology. Microbial Enzymes in Aquatic Environments. Berlin, Springer. 60-83.

Hungate, R. E. (1969). A roll tube method for cultivation of strict anaerobes. Methods in Microbiology. New York, Academic Press.

King, G. M. (1986). Characterization of $\beta$-glucosidase activity in intertidal marine sediments. AEM 51(2): 373-380.

Klok, J., M. Baas, H. C. Cox, J. W. De Leeuw, W. I. C. Rijpstra and P. A. Schenck (1984). Qualitative and quantitative characterization of the total organic matter in a recent marine sediment (Part II). Org. Geochem. 6: 265-278.

Meyer-Reil, L.-A. (1987). Seasonal and spatial distribution of extracellular enzymatic activities and microbial incorporation of dissolved organic substrates in marine sediments. Appl. Environ. Microbiol. 53(8): 1748-1755.

Meyer-Reil, L.-A. (1991). Ecological aspects of enzymatic activity in marine sediments. Microbial Enzymes in Aquatic Environments. Berlin, Springer. 84-95.

Moers, M. E. C., J. W. De Leeuw, H. C. Cox and P. A. Schenck (1988). Interaction of glucose and cellulose with hydrogen sulphide and polysulphides. Org. Geochem. 13(4-6): 1087-1091.

Priest, F. G. (1992). Synthesis and secretion of extracellular enzymes in bacteria. Microbial Degradation of Natural Products. New York, VCH. 1-26.

Rogers, H. J. (1961). The dissimilation of high molecular weight substances. The Bacteria. New York, Academic Press. 257-318.

Somville, M. (1984). Measurement and study of substrate specificity of exoglucosidase activity in eutrophic water. $A E M$ 48(6): 1181-1185.

White, C. A. and J. F. Kennedy (1988). The carbohydrate-directed enzymes. Carbohydrate Chemistry. Oxford, Clarendon Press. 343-377. 


\section{Appendix A: NMR Acquisition and Processing Parameters (13C and 2D Experiments)}

NOTE: Standard Brucker software was used for acquisition and processing of all spectra. This list of parameters is not intended to be a guide or explanation of the ${ }^{13} \mathrm{C}$ and $2 \mathrm{D}$ experiments; consult the literature on NMR spectroscopy cited in Chapter 2 for information on these topics. This list of experimental parameters may be useful for those who have hands-on experience with NMR spectrometers; Brucker manuels should be consulted for explanations of abbreviations and experiments.

\section{Chapter 2:}

gentiobiose COSY 45 (Fig. 2.7)

$$
\begin{aligned}
& \text { AU PROG: } \\
& \text { COSYHG.AUR } \\
& \text { DATE 26-9-91 } \\
& \begin{array}{lc}
\text { SI2 } & 1024 \\
\text { SI1 } & 512 \\
\text { SW2 } & 798.722 \\
\text { SW1 } & 399.361 \\
\text { NDO } & 1
\end{array} \\
& \begin{array}{lr}
\text { WDW2 } & \text { S } \\
\text { WDW1 } & \text { S } \\
\text { SSB2 } & 0 \\
\text { SSB1 } & 0 \\
\text { MC2 } & \text { M } \\
\text { PLIM } & \text { ROW: } \\
\text { F1 } & 5.547 P \\
\text { F2 } & 2.891 P \\
\text { AND } & \text { COLUMN: } \\
\text { F1 } & 5.547 P \\
\text { F2 } & 2.891 P
\end{array} \\
& 6.0000000 \\
& \text { 29L } \\
& 340020000 \\
& 4.50 \\
& .0000030 \\
& .0800000 \\
& 0.0^{2.30} \\
& 0.0 \\
& 785.00 \\
& 25 \\
& .0012520
\end{aligned}
$$

isomaltose COSY 45 (Fig. 2.8) AU PROG:

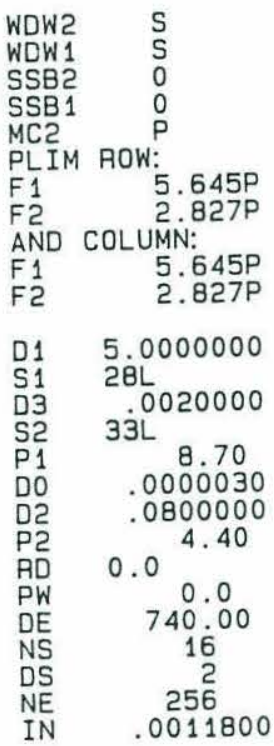


isomaltose ${ }^{13} \mathrm{C}$ (Fig. 2.10)

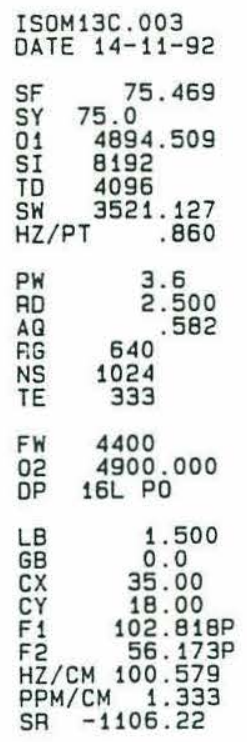

isomaltose HETCOR (Fig. 2.12)

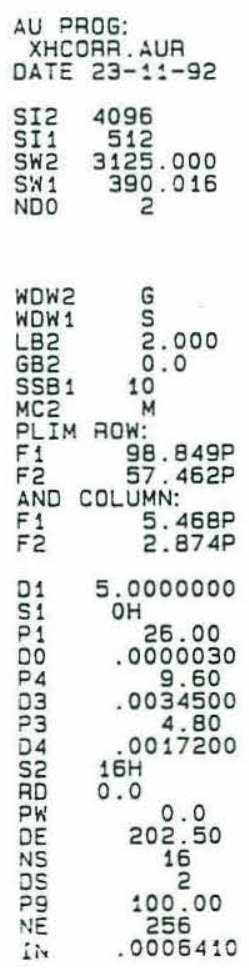

isomaltose DEPT (Fig. 2.11)

$$
\begin{aligned}
& \text { ISODEPT . } 045 \\
& \text { AU PROG: } \\
& \text { DEPT, AUR } \\
& \text { DATE } 13-11-92 \\
& \text { SF } 75.75 .469 \\
& 014952.706 \\
& \text { SI } 8192 \\
& \text { TD } 4096 \\
& \begin{array}{ll}
\mathrm{SW} & 3424.65 \mathrm{E} \\
\mathrm{HZ}: \mathrm{PT} & .836
\end{array} \\
& \begin{array}{lc}
\text { PW } & 0.0 \\
\text { RD } & 0.0 \\
\text { AG } & 640.599 \\
\text { RG } & 640 \\
\text { NS } & 64 \\
\text { TE } & 333
\end{array} \\
& \text { FW } 4300 \\
& \begin{array}{l}
\text { O2 } \\
\text { DP } 16 \mathrm{~L} \text { D } 00
\end{array} \\
& \text { LB } \quad 1.500 \\
& \text { GB } \quad 0.0 \\
& \text { CY } 5.00 \\
& 1 \quad 103.096 \mathrm{P} \\
& \text { F2, } 57.729 \\
& \mathrm{HZ} \text { ICM } 97.823 \\
& \mathrm{PPM} / \mathrm{CM} 1.296 \\
& \text { SR }-1109.65
\end{aligned}
$$

maltose COSY 45 (Fig. 2.13)

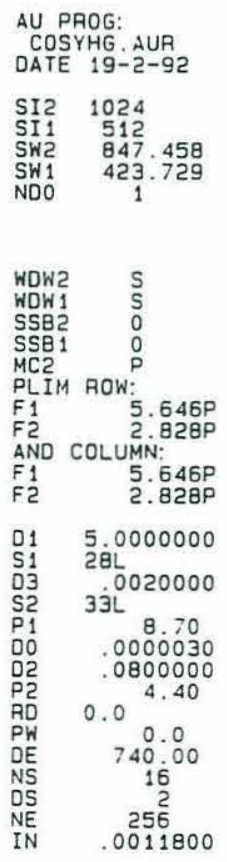


maltotriose COSY 45 (Fig. 2.14)

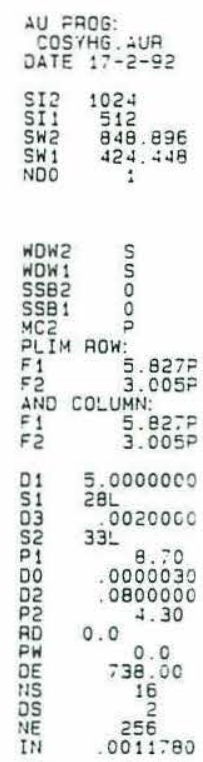

pullulan COSY (Fig. 2.18)

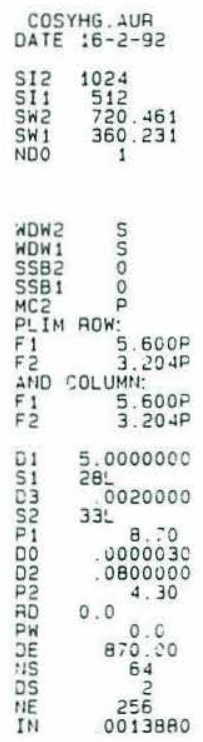

amylose ${ }^{13} \mathrm{C}$ (Fig. 2.16)

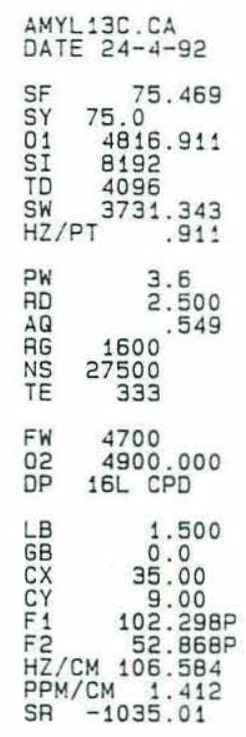

pullulan ${ }^{13} \mathrm{C}$ (Fig. 2.19)

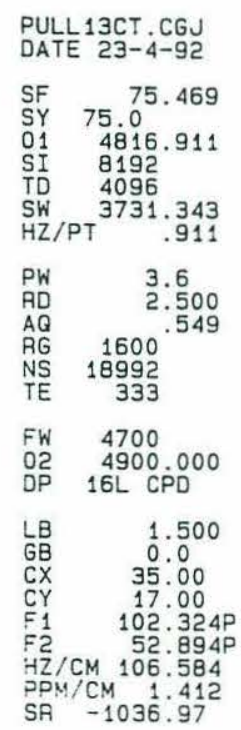


pullulan HETCOR (Fig. 2.20)

AU PROG:

XHCORA. AUR
DATE 5-12-92

$\begin{array}{lr}\text { SI2 } & 4096 \\ \text { SI1 } & 512 \\ \text { SW2 } & 3424.658 \\ \text { SW1 } & 841.751 \\ \text { ND0 } & 2\end{array}$

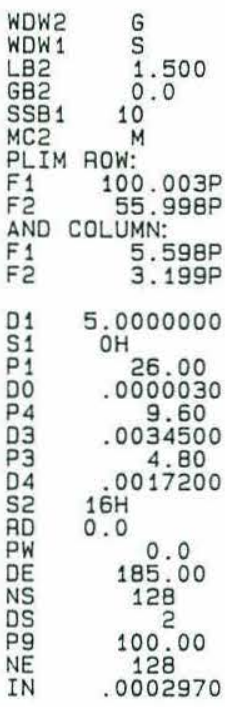

F3 COSY (Fig. 3.12)

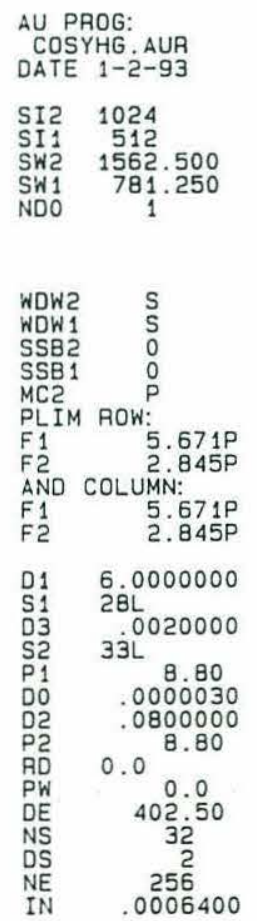

Chapter 3:

F2 COSY (Fig. 3.9)

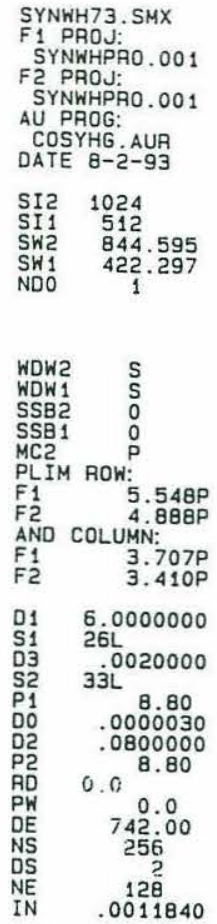

F3 ${ }^{13} \mathrm{C}$ (Fig. 3.14)

$$
\begin{array}{lc}
\text { DATE } & 12-2-93 \\
\text { SF } & 75.469 \\
\text { SY } & 75.0 \\
\text { O1 } & 4760.266 \\
\text { SI } & 8192 . \\
\text { TD } & 4096 \\
\text { SW } & 3546.099 \\
\text { HZ/PT } & .866 \\
\text { PW } & 3.6 \\
\text { RD } & 2.500 \\
\text { AQ } & 1600.578 \\
\text { AG } & 1600 \\
\text { NS } & 51321 \\
\text { TE } & 333 \\
\text { FW } & 4500 \\
\text { O2 } & 4900.000 \\
\text { DP } & 16 L \\
\multicolumn{2}{c}{P 0} \\
\text { LB } & 1.500 \\
\text { GB } & 0.00 \\
\text { CX } & 15.00 \\
\text { CY } & 12.00 \\
\text { F1 } & 100.667 P \\
\text { F2 } & 53.691 P \\
\text { HZ/CM } & 236.349 \\
\text { PPM } & 3.13 \\
\text { SR } & -1061.35 \\
\text { SH } &
\end{array}
$$


F3 DEPT (Fig. 3.15)

F3 HE'TCOR (Fig. 3.16)
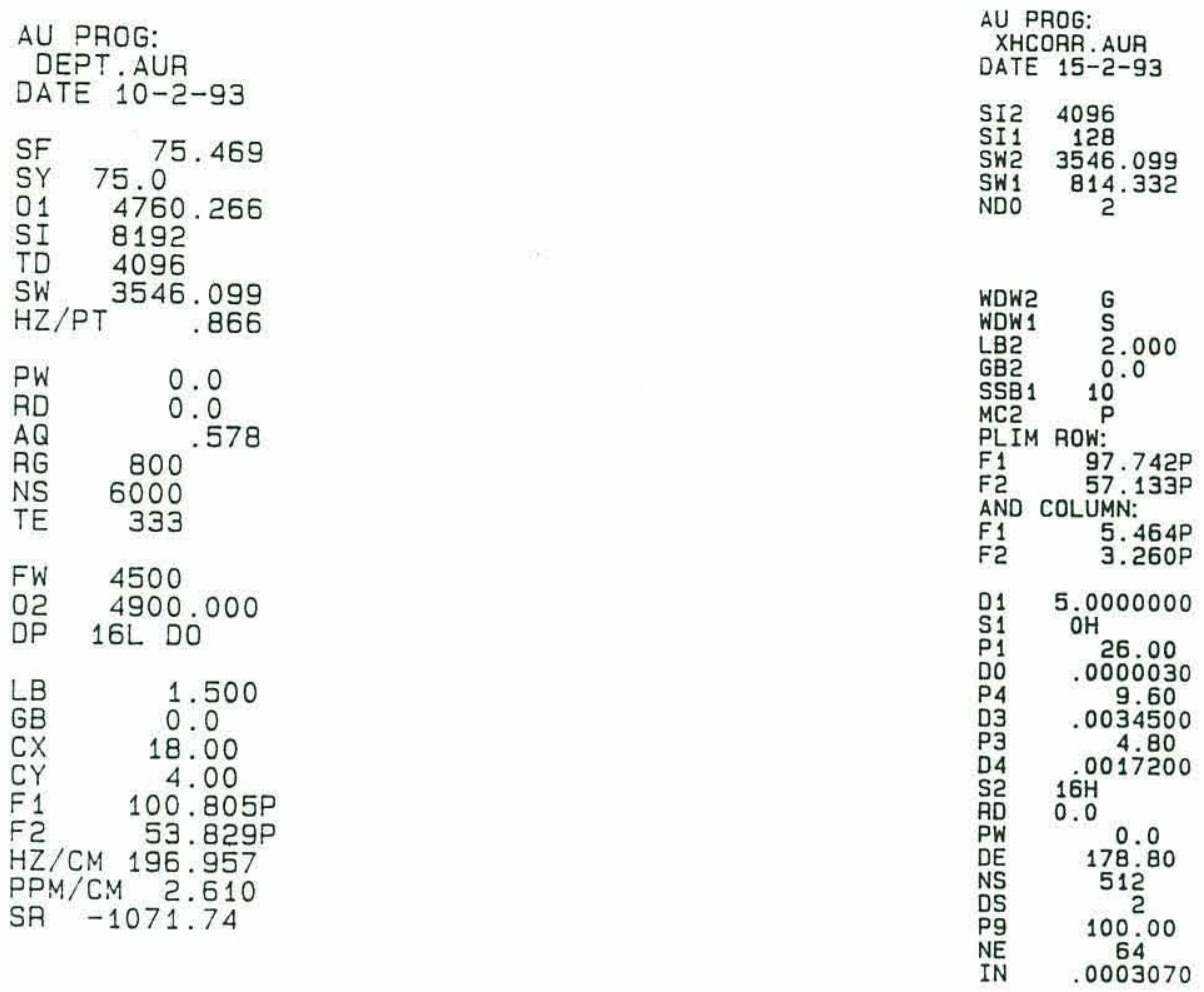


\section{Appendix B: Survey of Phytoplankton Carbohydrates}

TYPE:

structural

-harvested, sonicated to remove cell contents, dried. Hydrolyzed w/ $1.8 \mathrm{~N} \mathrm{HCl}$ at $100{ }^{\circ} \mathrm{C}$. Found glucose, galactose, mannose, xylose, fucose, rhamnose (in most species), little to no arabinose, ribose (Hecky et al., 1973)

-heated dried algae in $\mathrm{NaOH}, \mathrm{H}_{2} \mathrm{SO}_{4}$; ppt'd w/ EtOH. Found 90\% (1-3) $\beta$-xylan, $10 \%$ $\beta(1-4)$ glucan.(Maeda et al., 1990)

-after extraction w/ acid, residue extracted w/ alkali. Hydrolyzed, found complex mix of monomers, including rhamnose, fucose, ribose, xylose, mannose, galactose, glucose. (different $\%$ in different species). (The two fractions accounted for $100 \%$ of total carbohydrate). Didn't analyze for uronic acids. (Haug and Myklestad, 1976)

-insoluble carbohydrate (after acid and alkali extraction) was typically $32-52 \%$ of organic matter for samples high in dinoflagellates, which are known to have a cellulose-type cell wall. (Haug et al., 1973)

-partial degradation of cleaned cell walls yielded cellodextrins (DP2-4), laminaribiose, and laminaritriose (ie, both $\beta(1,4)$ and $\beta(1,3)$ linkages present). (Nevo and Sharon, 1969)

-coccolith polysacc isolated; found uronic acids, investigated $\mathrm{Ca}++$ binding, isolated similar polysaccharide from Black Sea sediment $E$. huxleyi (estimated age ca. $1000 \mathrm{yr}$ ) (De Jong et al., 1976)

\section{storage:}

-freeze-dried alga extracted with $.05 \mathrm{M} \mathrm{H}_{2} \mathrm{SO}_{4}$, neutralized, dialyzed. $16.2 \%$ yield, $>99 \%$ glucose, trace mannose. Avg. DP of 106 (determined by measuring reducing power). Mainly $\beta(1-6)$ linked w/ $(1,3)$ branch points that have $(1,6)$ sidechains (Varum et al., 1986)

-freeze-dried alga extracted $2 \mathrm{x}$ w/.1N H2SO4 to give total glucan. Found only trace quantities of other sugars, and only small amounts of glucose remained in reside. $\beta(1,3)$ linked; believe this extract represents storage prod. Didn't analyze for uronic acids. (Haug and Myklestad, 1976) (Myklestad, 1974)

-extracted lyophilized mixed-species (net tow) w/ .1 $\mathrm{N} \mathrm{H}_{2} \mathrm{SO}_{4}$; got 95-98\% glucose. Diatoms seem to have glucose principally as storage product; w/ dinoflagellates, acid- and alkali-soluble material v similar in composition.(Haug et al., 1973)

-indications of $\beta(1,3)$ glucose polysaccharide (Handa, 1969)

-extracted cells w/ .05 $\mathrm{M} \mathrm{H}_{2} \mathrm{SO}_{4}$ at $4{ }^{\circ} \mathrm{C}$ for $1 \mathrm{hr}$; found a $\beta(1,3)$ glucan $w /$ branches at positions 2 and 6. (Paulsen and Myklestad, 1978)

\section{excreted}

-filtered and rotovaped medium, dialysed, lyophilized: contained sulfate half-esters, rhamnose, fucose, arabinose, galactose (Myklestad et al., 1972) Followed up w/ periodate oxidation, methylation: found branched structure, $w /$ fucose and galactose on inner and outer, rhamnose on outer parts. Rhamnose generally $(1,2)$, galactose $(1,4)$ and $(1,3)$, 
fucose is $(1,3)$ and at branch points. Previous report of arabinose in error; rhamnose:fucose:galactose $=4: 75: 21$ (Smestad et al., 1974)

-Dialysed, lyophilized, dissolved in $\mathrm{H}_{2} \mathrm{O}$, ppt'd w/ EtOH, lyophilized again. Contained fucose, rhamnose, mannose, glucose, xylose, glucuronic acid, galactose (34:15:19:16:6:9:trace molar ratio). Sulfate also found, total production ca. $30 \mathrm{mg} / \mathrm{dm}^{3}$. Fractionation showed at least 3 polymers: a glucan, a heteropolymer w/o glucose, and a polymer w/ mannose and fucose. Glucan had primarily $(1,3)$ links, w/ some branches through $\mathrm{C} 1, \mathrm{C} 3$, and $\mathrm{C} 6$. Glucose probably chrysolaminarin; may be derived from dead cells (10 wk total incubation time before cells harvested and removed) (Percival et al., 1980)

-12-day cultures; got 4.5-27 mg/L carbohydrate from Chaetoceros species. Found rhamnose, fucose, galactose in all polysaccharides; xylose, mannose, and glucose in two of four species. Polymers were homogenous (free-boundary electrophoresis); structural analysis (unspecified) indicates high degree of branching. Amount of exopolysacc $>$ total amounts of cellular, alkali-sol carbohydrate. Since cultures so fresh, quantities not likely due to cell lysis. (Haug and Myklestad, 1976)

-production in Chaetoceros species ranged from 6-27 mg/L. Ratio of extracellular to cellular carbohydrate ranged from .29 to 1.25 for Chaetoceros species; other species were lower. (Myklestad, 1974)

-cells removed from media by centrifugation. Medium dialyzed, concentrated, lyophilized. Analyzed by ionophoresis, paper chromatography, gas-liq chromatography. Found primarily rhamnose, also mannose, fucose, xylose, galactose, glucose; arabinose and ribose and unknowns in exosaccharides from some species. Glucuronic test positive for all species checked. Nitzschia frustulum yielded highest quantities of exopolysaccharide. Production of polymer started in period between log and stationary phase, and continued for $14 \mathrm{~d}$ (in a $21 \mathrm{~d}$ culture) Polysaccharide produced in high S\%o culture differed in solubility from those produced in low $\mathrm{S} \%$ o cultures. Found compositional differences in the two exopolysaccharides. (Allan et al., 1972)

-sulfated polysaccharide produced by diatom: moved as single anionic compound $w /$ free boundary electrophoresis. Sulfated, has rhamnose, fucose, galactose $(.3: 3.5: 1)$ Highly branched, w/ fucose in both furanose and pyranose forms. Galactose present mainly in inner part of molecule. (Smestad et al., 1975)

-filtered culture medium, determined carbohydrate w/ N-ethyl carbazole. Maximum concentration ranged from $10-123 \mathrm{mg} / \mathrm{L}$, depending on species. (Guillard and Wangersky, 1958)

-investigated kinetics of ${ }^{14} \mathrm{C}$-labeled particulate production and extracellular release in (zoopl mesh) filtered water from three sites [not carbohydrate-specific production] (Lancelot, 1979)

-measured total carbohydrate, polysacc produced in batch cultures; rate ranged from 42 $\mathrm{pg} /$ cell/day for $\log$ to $20 \mathrm{pg} / \mathrm{cell} /$ day for stationary phase cultures (Myklestad et al., 1989)

-mild acid hydrolysis, column chromatography [particulars NOT specified] used to isolate 3-0- ( $\alpha$-D-glucopyranosyluronic acid)-L-galactopyranose; structure determined by NMR (Jaseja et al., 1989) 
-from culture in stationary phase, isolated exopolysaccharide by dialyzing, lyophilizing, hydrolysis w/ $2 \mathrm{M}$ TFA at $100^{\circ} \mathrm{C}$ for $2 \mathrm{hr}$. Used DE-52 ion exchange resin to separate neutral, anionic components, then used Sephadex G10 to do size separation. Used NMR, GLC-ei-MS to determine structure of 3-0- ( $\alpha$-D-glucopyranosyluronic acid)-Lgalactopyranose (Geresh et al., 1990)

-mild acid hydrolysis, anion exchange chromatography, GPC to isolate sulfated sugar fraction, desulfated, converted to alditol acetates, used IR and NMR to determine structure as D-galactose 6-sulfate, D-glucose 6-sulfate, D-galactose 3-sulfate. (Lupescu et al., 1991)

-sequence of ppt steps used to isolate exopolysaccharide; a high MW heteropolymer (1$\left.10 \times 10^{\wedge} 6\right), 42 \%$ hexose: $30 \%$ pentose: $8.5 \%$ uronic acid: $9 \%$ ester sulfate: $1-2 \%$ amino acid. Glucose and galactose make up hexoses, xylose is pentose. Galactose: xylose: glucose: uronic acid: sulfate is 2.12:2.42:1:1.2:2.61. (all this known from previous wk). Determined structure of a methylated glucuronic acid by ci-MS and a variety of other techniques. (Kieras et al., 1976)

-radiolabeled extracellular productss dialyzed, hydrolyzed, paper chromatography vs. stds. Found galactose, glucose, mannose, arabinose, xylose, ribose, rhamnose, hexuronic acid, and unknown. Cold- and warm-water species had same sugars (except arabinose), but relative proportions different. (Guillard and Hellebust, 1971)

-medium filtered, concentrated, lyophilized, acetylated derivatives, GC: found galactose, also mannose, glucose, xylose, arabinose; little rhamnose (Vieira and Myklestad, 1986)

-total dissolved carbohydrate measured (method not specified) in a variety of species grown in culture. For Phaeodactylum, filtered medium pH-adjusted to 5.0, passed through $\mathrm{C} 18$ sep-pak, eluted w/ MeOH. Rotovaped, methanolysis, TMS, GC. Neutrals, carboxylated, amino sugars detected; sulfate esters would've been desulfated, measured w/ others. Dissolved carbohydrates ranged from ca. $2-15 \mathrm{mg} \mathrm{C} / 1$ medium. Total of Phaeodactylum carbohydrate in surface-active material reached a max of $1.4 \mathrm{mg} / \mathrm{l}$ extract. (Frew et al., 1990)

-cells removed by centrifugation, medium concentrated, dialyzed (10-14kD cutoff), separated by gel permeation, etc. In soil cryptomonas, found fucose, rhamnose, xylose, mannose, glucose, galactose-uronic, glucose-uronic, trace of 3-O-Me galactose. Fract $\mathrm{A}$ was 1,3-linked galactose, 1,4-linked galactose-uronic. Fract B had more sulfate, was more highly branched, had greater compositional variety. (Paulsen et al., 1992)

\section{extracellular mucilage}

-diatom grows in colonies surrounded by mucilage tubes; tube polymer consists of $72 \%$ carbohydrate, $22.5 \%$ sulphate, $7.5 \%$ protein. Carbohydrate consisted of $20-22 \%$ uronic acid, $17-20 \%$ anhydrogalactose. Polymer moved as single anionic compound w/ free-bdry electrophoresis. Complete hydrolysis gave mannose, mannuronic acid, xylose, and an unknown sugar. Main part of mannose $(1,3)$ linked, some has branch points at $\mathrm{C} 2$ and $\mathrm{C} 3$, some present as non-reducing endgroups. (mannose derivatives also represent mannuronic acid). Xylose present as non-reducing endgroups, and as $(1,2)$ and $(1,4)$ links. Unknown is probably 3,6-anhydro-2-O-methyl-hexitol triacetate, either $(1,4)$ or $(1,5)$ linked. Sulfate on C2 of mannose or mannuronic acid, or at C4 (Paulsen et al., 1978)

-red algae mucilage found to be sulfated polysacc w/ xylose and glucuronic acid, and 
galactose, glucose, rhamnose, and 3-O-methyl xylose. (Fareed and Percival, 1977)

- red algae mucilage: xylose, glucose, D- and L-galactose, 3-O-methylxyl, 3- and 4-Omethyl galactose, glucuronic acid (3:1:2.5:.13:.13:.8). Also found 2-O-methylhexose, 2-Omethylglucuronic acid, 2,4-di-O-methylgal. Also ca $10 \%$ sulfate half-esters. Found 1,3 and 1,4 links. MW estimated ca $4 \times 10^{\wedge} 6$ and $5 \times 10^{\wedge} 6$. (Percival and Foyle, 1979)

\section{(general) cellular/extracted}

-acid hydrolysate: extracted w/. $1 \mathrm{~N} \mathrm{H}_{2} \mathrm{SO}_{4}$, upped to $1 \mathrm{~N}$, acetylated+GC

extracted remainder $\mathrm{w} / 1 \mathrm{~N} \mathrm{H}_{2} \mathrm{SO}_{4}$, acetylated + $\mathrm{GC}$ (Myklestad et al., 1972)

-sonicated cells in water, centrifuged to remove cell walls: Hydrolyzed w/ $1.8 \mathrm{~N} \mathrm{HCl}$ at $100^{\circ} \mathrm{C}$. Much glucose, some galactose, mannose, xylose, rhamnose, fucose; little to no ribose, arabinose (Hecky et al., 1973)

-hot-alkali extract: $5 \%$ yield from cells, v soluble in $\mathrm{H}_{2} \mathrm{O}$. Mannose, $27 \%$ glucuronic acid, $7.5 \%$ ester sulphate[though paper is confusing with respect to composition]. Found $(1,3)$ and $(1,2)$ linkages (Ford and Percival, 1965)

-sequential extraction w/ water, alkali (cold $\mathrm{H}_{2} \mathrm{O}$, hot $\mathrm{H}_{2} \mathrm{O}, \mathrm{NaOH}$ ): got glucose, mannose, and fucose from water extract (after hydrolysis), mannose and uronic acid from alkali extract. (Percival et al., 1980)

-acid hydrolysate: glucose, galactose, ribose in all species, mannose in bacillariophyceae; xylose, rhamnose, fucose in sev. species (see table in paper). Didn't find large quantities of hexuronic acids (Parsons et al., 1961)

-diatoms refluxed in $\mathrm{MeOH}: \mathrm{H}_{2} \mathrm{O}$ (90:10) 2x, then refluxed in $\mathrm{H}_{2} \mathrm{O}$. EtOH added to aq. extract, dehyrated gummy mass $\mathrm{w} / \mathrm{MeOH}$ and ether extractions. Found glucose, trace of other monomers (probably contamination from extracellular extract). Alkali labile polymer yielded metasaccharinic acid, consistent w/ 1-3 linkage. In (hydrolyzed) residue after $\mathrm{H}_{2} \mathrm{O}$ extract, got same monomers as in exopolysacc: rhamnose, fucose, xylose, mannose, galactose, glucose, and 3 unknowns. Proportions of monomers different, though. (Allan et al., 1972)

-EDTA-soluble fraction of coccolith (coccoliths decalcified in 10\% EDTA, supernatant filtered, Bio-Gel P4, Amicon PM-10 filter, dialysis, lyophilized): acidic polysacc, single peak on DEAE-cellulose chromatog. Contained ester sulphate, galactose, glucose, mannose, rhamnose, ribose, arabinose, xylose, galacturonic acid, 2,3-di-O-Me rhamnose, 3-O-Me xylose, 6-O-Me mannose. (Fichtinger-Schepman et al., 1979)

-alkali-sol dinoflagellate extract contained large amounts of glucose, while alkali-soluble diatom extracts contained a comples mixture of monomers. (Haug et al., 1973)

-lyophilized phytoplankton were Soxhlet extracted $\mathrm{w} /$ benzene:MeOH, treated $\mathrm{w} / .01 \mathrm{~N}$ $\mathrm{HCl}$, and then $\mathrm{w} / .5 \mathrm{~N} \mathrm{NaOH}$. Collected solid residue, washed $\mathrm{w} / \mathrm{HCl}$, analyzed by solidstate 13C NMR. (Zelibor et al., 1988)

-developed new analytical techniques to quantitatively determine anhydroglycans, sulfated polysacc (derivatize to alditol acetates) (Stevenson and Furneaux, 1991)

-cold- and hot-water base extracts to obtain carrageenan. Has backbone of alternating 3- 
linked $\beta$-D galactose and 4-linked $\alpha$-D-galactose ca $50 \%$ of the $\beta$-galactose units are 4sulfated; $\alpha$-galactose units are principally 3,6-anhydro [all this was previously known]. Did further investigation into structural details of carageenan; looked into content and distribut of 'precursors' and sequences; char'd by NMR. (Knutsen et al., 1990)

-fucose-containing sulfated polysacc w/ anticoagulant properties. W/ methylation analysis, determined sulfate principally at $\mathrm{C} 4$ of 3-linked fucan backbone. Also has 2-O-linked galactosyl residues, 4-O-linked and 3,4-di-O- substituted glucosyluronic acid residues as minor components. Galactosyl residues might be in backbone; could be in sidechain. (Nishino et al., 1991)

-(general review) Brown algae: alginic acid (polymer of mannuronic and guluronic acids), fucans (fucose with other sugar residues and sulfate esters). Red algae: sulfated galactans (which have galactose, 3,6-anhydrogalactose). Green algae: complex polysacc, many of which are sulfated. "All algae" synthesize glucans as storage polysacc. (Percival and McDowell, 1990)

-Whole-cell carbohydrate composition: (determined via acid hydrolysis, GC) rhamnose, fucose, arabinose, xylose, mannose, glucose, trace of ribose, galactose, and arabinose. (Handa, 1969)

-whole-cell carbohydrate composition (acid hydrolysis, GC): glucose was most common monosaccharide in 7 of 8 species; rhamnose, fucose, ribose, xylose, arabinose, mannose, galactose also found. Significant differences in carbohydrate composition/content between species. (Brown, 1991)

\section{growth effects}

-increase in carbohydrate content per cell when nitrate is depleted(Myklestad and Haug, 1972)

-increase in carbohydrate/cell over time (ie, into lag phase of culture)(Myklestad and Haug, 1972)

-stationary phase: higher glucan content, though there are significant differences between species. Amount of alkali-sol (=cell wall) carbohydrate not $\mathrm{v}$ dependent on growth phase. (Haug and Myklestad, 1976)

-stationary-phase cultures in generally higher in 6-deoxy sugar (fucose, rhamnose) than log phase cultures (Haug and Myklestad, 1976)

- cells from different species harvested in log phase showed similar compositions, while cells in stationary phase differed between species. (Myklestad, 1974)

-salt concentrantion influenced cell size and number (lower S\%o $\Rightarrow>$ more, smaller cells; yield of exopoly increased six fold.) Higher nutrients $=>$ increase wt, exopolysaccharide yield. (Allan et al., 1972)

-found changes in protein:carbohydrate ratio w/ spring bloom, change in species composition (Haug et al., 1973)

-carbohydrate production did not parallel increase in cell \#s during exponential growth; maximum concentration was ca $3 \mathrm{mg} / \mathrm{L}$. Accumulation occurred in stationary phase 
(concentration ranged from ca $10-123 \mathrm{mg} / \mathrm{L}$, depending on species) (Guillard and Wangersky, 1958)

-cites evidence (studies of others, own field observations) that slowly growing phytoplankton in culture and in natural waters produce and release more extracellular carbohydrate than rapidly growing cells.(Burney et al., 1981)

-carbohydrate excreted during ALL phases of growth; composition distinct from reserve or cellwall polysaccharides. Eestimated/calculated rate of 42pg/cell/day in log phase, 20 $\mathrm{pg} / \mathrm{cell} / \mathrm{day}$ in stationary phase (Myklestad et al., 1989)

-rapidly growing cells produced relatively little carbohydrate; slow-growing cells produced more. Production also influenced by N:P ratio of medium. Especially at high N:P ratios, $C$. affinis produced large amount of exopolysaccharide. Production of exopolysaccharide continued even after cellular accumulation of carbohydrate had ceased. (Mykelstad, 1977)

-concentration of dissolved carbohydrate increased w/ time from .14 to $.4 \mathrm{mg} \mathrm{C} / \mathrm{L}$. No change in dissolved carbohydrate (extracellular) when culture transferred to dark. Cellular carbohydrate was lost before protein, lipids in dark; loss of carbohydrate was in watersoluble (presumably storage) carbohydrate, not cell wall carbohydrate. (Handa, 1969)

-Production of exopolysaccharide observed only in actively photosynthesizing cells; carbohydrate not due to leakage from dead/dying cells, or dissolution of gel-capsule around cell. $\mathrm{N}$ depletion in medium caused increase in exuded carbohydrate; carbohydrate produced in all phases of growth. (Vieira and Myklestad, 1986)

\section{SPECIES:}

(note: check (Painter, 1983) for further species; ca. 100 species mentioned)

\section{-diatoms (Bacillariophyceae):}

storage products: laminarin (Lee, 1980; Painter, 1983)

other polysacc: cellulose? chitin? complex extracellular mucilages, sulfated glucuronomannans (Lee, 1980; Painter, 1983)

Asterionella socialis (Allan et al., 1972)

Amphiprora paludosa (Allan et al., 1972)

Berkeleya rutilans (Paulsen et al., 1978)

Chaetoceros affinis (Myklestad and Haug, 1972; Myklestad et al., 1972; Smestad et al., 1974; Parsons et al., 1961; Haug and Myklestad, 1976)

(Myklestad, 1974; Myklestad et al., 1989; Mykelstad, 1977)

Chaetoceros brevis (Haug et al., 1973)

Chaetoceros calcitrans (Brown, 1991)

Chaetoceros curvisetus (Haug and Myklestad, 1976; Myklestad, 1974; Haug et al., 1973; Smestad et al., 1975; Frew et al., 1990)

Chaetoceros debilis (Haug and Myklestad, 1976; Myklestad, 1974; Haug et al., 1973)

Chaetoceros decipiens (Haug and Myklestad, 1976; Myklestad, 1974)

Chaetoceros gracilis (Brown, 1991)

Chaetoceros laciniosus (Haug et al., 1973)

Chaetoceros similis (Haug et al., 1973)

Chaetoceros socialis (Haug and Myklestad, 1976; Myklestad, 1974; Haug et al., 1973)

Corethron hystrix (Haug and Myklestad, 1976; Myklestad, 1974) 
Coscinodiscus sp. (Parsons et al., 1961)

Coscinodiscus nobilis (Percival et al., 1980)

Cyclotella cryptica (estuarine) (Hecky et al., 1973)

*. Cyclotella nana (Allan et al., 1972)

Cyclotella stelligera (freshw) (Hecky et al., 1973)

Cyclotella sp. (Guillard and Wangersky, 1958)

Cylindrotheca fusiformis (Allan et al., 1972)

Melosira granulata (freshw) (Hecky et al., 1973)

Melosira nummuloides (estuarine) (Hecky et al., 1973)

Navicula incerta (Allan et al., 1972)

Navicula pelliculosa (freshw) (Hecky et al., 1973)

Nitzschia angularis (Allan et al., 1972)

Nitzschia brevirostris (estuarine) (Hecky et al., 1973)

Nitzchia closterium (Brown, 1991)

Nitzschia frustulum (Allan et al., 1972; Frew et al., 1990)

Phaeodactylum tricornutum (Ford and Percival, 1965; Parsons et al., 1961; Frew et al., 1990; (Brown, 1991)

Rhizosolenia imbricata (Haug et al., 1973)

Skeletonema costatum (Parsons et al., 1961; Myklestad, 1974; Allan et al., 1972; Haug et al., 1973; Mykelstad, 1977; (Handa, 1969; Paulsen and Myklestad, 1978; Brown, 1991)

Thalassionema nitzschioides (Haug et al., 1973)

Thalassiosira gravida (Haug and Myklestad, 1976; Myklestad, 1974; Haug et al., 1973)

Thalassiosira fluviatilis (Myklestad, 1974)

Thalassiosira pseudonana (Brown, 1991)

-coccoliths (Prymnesiophyceae):

storage products: chrysolaminarin in vesicles in cell posterior (Lee, 1980)

Emiliania huxleyi (Varum et al., 1986; Fichtinger-Schepman et al., 1979;

De Jong et al., 1976)

Isochrysis galbana (Guillard and Wangersky, 1958; Frew et al., 1990;

Brown, 1991)

Pavlova lutheri (Brown, 1991)

Pavolva salina (Brown, 1991)

Phaeocystis pouchetii (Haug et al., 1973; Guillard and Hellebust, 1971)

Prymnesium parvum (Guillard and Wangersky, 1958)

-dinoflagellates (Dinophyceae):

storage products: starch (Lee, 1980; Painter, 1983)

Amphidinium carteri (Parsons et al., 1961; Guillard and Wangersky, 1958)

Ceratium fusus (Haug et al., 1973)

Ceratium longipes (Haug et al., 1973)

Ceratium tripos (Haug et al., 1973)

Exuviella sp. (Parsons et al., 1961)

Gonyaulax tamarensis (Haug et al., 1973)

Gymnodinium sp. (Guillard and Wangersky, 1958)

Peridinium trochoideum (Haug et al., 1973)

Peridinium westii (Nevo and Sharon, 1969)

-green algae (Chlorophyceae):

(green algae sp.) (Percival and McDowell, 1990) 
storage product: starch (Lee, 1980), also inulin, possibly laminarin (Painter, 1983) other sacc: true cellulose, modified cellulose, mannans, gluco-mannans, xylans, pectic acid, complex hemicelluloses, sulfated mucilages (Painter, 1983)

Ankistrodesmus densus (Vieira and Myklestad, 1986)

Botryococcus braunii (Zelibor et al., 1988; Berkaloff et al., 1983)

Bryopsis maxima (Maeda et al., 1990)

Chlamydomonas sp. (Zelibor et al., 1988)

Chlamydomonas " $Y$ " (Guillard and Wangersky, 1958)

Chlorella pyrenosidosa (Zelibor et al., 1988)

Chlorella stigmatophora (Frew et al., 1990)

Chlorella sp. (Zelibor et al., 1988; Guillard and Wangersky, 1958)

Chlorococcum sp. (Guillard and Wangersky, 1958)

Closterium sp. (Zelibor et al., 1988)

Dunaliella euchlora (Guillard and Wangersky, 1958)

Dunaliella salina (Parsons et al., 1961)

Dunaliella tertiolecta (Zelibor et al., 1988)(Brown, 1991)

Hydrodictyon sp. (Zelibor et al., 1988)

Nannochloris atomus (Brown, 1991)

Scenedesmus sp. (Zelibor et al., 1988)

Scenedesmus obliquus (Zelibor et al., 1988)

Tetraselmis maculata (Parsons et al., 1961)

Zygnema sp. (Zelibor et al., 1988)

-golden-brown algae (Chrysophyceae):

storage product: chrysolaminarin (Lee, 1980); laminarin? (Painter, 1983)

other sacc: complex sulfated calcium-binding heteroglycans (Painter, 1983)

Monochyrsis lutheri (Parsons et al., 1961)

Olisthodiscus luteus (Frew et al., 1990)

Syracosphaera carterae (Parsons et al., 1961)

-red algae (Rhodophyceae)

storage product: floridean starch (similar to amylopectin) (Lee, 1980; Painter, 1983)

cell wall: cellulose (Lee, 1980)

other sacc: true cellulose doubtful; mannans, xylans, sulfated galactans, complex extracellular mucilages (Painter, 1983)

(red algae sp.) (Stevenson and Furneaux, 1991; Geresh et al., 1990;

Percival and McDowell, 1990)

Porphyridium aerugineium (Percival and Foyle, 1979)

Porphyridium cruentum (Kieras et al., 1976; Percival and Foyle, 1979)

Porphyridium sp. (Lupescu et al., 1991; Frew et al., 1990)

Rhodella maculata (Fareed and Percival, 1977)

Rhodella reticulata (Jaseja et al., 1989)

(agars)

\section{-brown algae (Phaeophyceae)}

storage product: laminarin (Lee, 1980; Painter, 1983)

cell walls: generally 2 layers. cellulose is main structural skeleton, alginic acid $(\beta(1,4)$ manuronic acid, variable amount of guluronic acid attached at $\mathrm{C} 1, \mathrm{C} 4)$ and fucoidin $(\alpha(1,2)$ sulfated fucose, lesser amount of $\alpha(1,4)$ sulfated fucose) are amorphous. Relative amounts of alginic acid, fucoidin, vary between species, plant part, environ. (Lee, 1980)

other sacc: cellulose, lichenan, alginate, fucoidan, sulfated hexuronoxylofucans, 
complex sulfated heteroglycans (Painter, 1983)

Ecklonia kurome (Nishino et al., 1991)

(brown algae sp.) (Percival and McDowell, 1990)

-Prasinophytes:

Tetraselmis chui (Brown, 1991)

Tetraselmis suecica (Brown, 1991)

-Eustigmatophyte:

Nannochloropsis oculata (Brown, 1991)

-Cryptophyte:

Chroomonas salina (Brown, 1991)

-(to be classified)

Actinocyclus sp. (Guillard and Wangersky, 1958)

Distephanus speculum (Haug et al., 1973)

Furcellaria lumbricalis (Knutsen et al., 1990)

Melosira sp. (Guillard and Wangersky, 1958)

Monochrysis lutheri (Guillard and Wangersky, 1958)

Nitzschia brevirostris (Guillard and Wangersky, 1958)

Pyraminomonas sp. (Guillard and Wangersky, 1958)

Rhodomonas sp. (Guillard and Wangersky, 1958)

Cryptomonas (Paulsen et al., 1992) [[soil species!!]]

\section{METHODS:}

-phenol- $\mathrm{H}_{2} \mathrm{SO}_{4}$ (Myklestad and Haug, 1972)

-phenol- $\mathrm{H}_{2} \mathrm{SO}_{4}$, electrophoresis (demonstrated that polysacc was anionic), acetylated, GC; paper chromatography.(Myklestad et al., 1972)

-periodate oxidation, methylation; position of sulfate estimated by IR spectra (Smestad et al., 1974)

-hydrolysis, alcohol ppt, cellulose chromatography, paper chromatography, Smith degradation, methylation, GC (Ford and Percival, 1965)

-hydrolysis of whole cell (apparently) with $\mathrm{H}_{2} \mathrm{SO}_{4}$, paper chromatography. (Parsons et al., 1961)

-hydrolysis of whole cell w/ .05 $\mathrm{M} \mathrm{H}_{2} \mathrm{SO}_{4}$, neutralization/dialysis/lyophilized, $\mathrm{GC}$ of alditol acetates, methylation analysis, periodate oxidation, Smith degradation, 13C NMR $(400=100 \mathrm{MHz})$ (Varum et al., 1986)

-hydrolysis w/ $1.8 \mathrm{~N} \mathrm{HCl}$, Mopper's sugar analyzer [borate complexes?](Hecky et al., 1973)

-dialyse, lyophilize, hydrolyze, fractionate into neutral and acidic fractions. alditol acetates, GC-MS, EI-MS, chrom. on DE-52 cellulose, methylation, periodate oxidation. (Percival et al., 1980)

-hot water treatment, dil. alkali and DMSO soluble. GPC, Smith degradation, alditol 
acetates, TMS derivs., GC, TLC, enzymatic hydrol., 1H NMR (90 MHz), IR (Maeda et al., 1990)

-(Myklestad, 1974) same methods as (Haug et al., 1973) (ditto (Mykelstad, 1977))

- $\mathrm{MeOH}: \mathrm{H}_{2} \mathrm{O}$ extract (discarded), $\mathrm{H}_{2} \mathrm{O}$ extract, spectrophotometric determination of carbohydrate concentration, methoxy function measurement, sulfate estimated by perchloric acid reflux and titration, uronic acid determined by carbazole reaction and 530nm absorbtion, alkaline degradation, paper chromatography, GLC, ionophoresis (Allan et al., 1972)

-isolated EDTA-soluble acidic polysacc from coccoliths. DEAE-cellulose chromatography, 2D cellulose-electrophoresis, polyacrylamide electrophoresis, ele. analysis, pyr.-MS, IR, $360 \mathrm{MHz}$ NMR (only determined - $\mathrm{CH} 3$ group at $\delta=1.3$ from deoxy hexose), turbidimetric determination of sulphate esters, paper chromatography, glc, glc-MS of methanolysates and hydrolysates. (Fichtinger-Schepman et al., 1979)

-lyophilized whole net-tow plankton. Extracted $2 \mathrm{x}$ w/ $.1 \mathrm{~N} \mathrm{H}_{2} \mathrm{SO}_{4}$; residue extracted $2 \mathrm{x}$ w/ .1 N NaOH. phenol- $\mathrm{H}_{2} \mathrm{SO}_{4}$. Estimated insoluble carbohydrate as difference between total and soluble. (measured insoluble in 2 cases by strong acid hydrolysis.) Alditol acetate determination of monomer comp. (Haug et al., 1973)

-free-boundary electrophoresis, complete hydrolysis, ion-exch chromatography, carbonCelite chromatography, alditol acetates, ei-MS, ci-MS, IR, methylation unsuccessful (apparently this often happens with polysaccharides which contain both sulphate and uronic acid; couldn't completely methylate desulphated polymer, either. Could methylate after desulphation and reduction.) (Paulsen et al., 1978)

-free boundary electrophoresis, complete acid hydrolysis, alditol acetates, methylation, GLC-MS. (Smestad et al., 1975)

-filtered medium, used N-ethyl carbazole test to determine carbohydrate (Guillard and Wangersky, 1958)

-liquid scintillation counting of particles and filtrate (not carbohydrate specific) (Lancelot, 1979)

-solid-state ${ }^{13} \mathrm{C}$ NMR used on 'alghumin' and 'hydrolyzed alghumin' isolated from green algae by sequential Soxhlet, .01N HCl, .5N NaOH, acidification to $\mathrm{pH}$. (Zelibor et al., 1988)

-'method of Strickland and Parson (1972)' used to determine total extracellular carbohydrate, and to determine total exopolysaccharide (after dialysis) (Myklestad et al., 1989)

-new techniques to derivatize 3,6-anhydrogalactans w/o destroying them. Also can produce partially methylated alditol acetates from sulfated polysaccharides. (Stevenson and Furneaux, 1991)

-used mild acid hydrolysis, column chromatography [unspecified] to isolate disacc from medium. 300MHz NMR $\left({ }^{1} \mathrm{H},{ }^{13} \mathrm{C}\right)$ used to determine structure (Jaseja et al., 1989) 
-from culture in stationary phase, isolated exopolysacc by dialyzing, lyophilizing, hydrolysis w/ $2 \mathrm{M} \mathrm{TFA}$ at $100^{\circ} \mathrm{C}$ for $2 \mathrm{hr}$. Used DE-52 ion exch resin to separate neutral, anionic components, then used Sephadex G10 to do size separation. Did total hydrolysis to determine monomer composition of polysaccharide, TLC, HPLC (ri detector), GCL, GCMS, FAB-MS. Used NMR, GLC-ei-MS to determine structure of 3-0- ( $\alpha$-Dglucopyranosyluronic acid)-L-galactopyranose (Geresh et al., 1990)

-polysaccharide hydrolyzed w/ .1M TFA for $3 \mathrm{hr}$ at $100^{\circ} \mathrm{C}, \mathrm{DE}-52$ ion exch, Sephadex G10, TLC to determine correct fractions to apply to Biogel P2 column, collected, lyophilized, desulfated by treatment $\mathrm{w} / 2 \mathrm{M} \mathrm{HCl}$ for $2 \mathrm{hr}$ at $100^{\circ} \mathrm{C}$. Alditol acetates, GLC. IR, NMR (300 MHz, used to determine position of sulfate, relative ratio of saccharide types) (Lupescu et al., 1991)

-dried, milled, treated $\mathrm{w} / .1 \mathrm{M} \mathrm{HCl}$ for $30 \mathrm{~min}$ at $4{ }^{\circ} \mathrm{C}$, washed for $4 \mathrm{hr} \mathrm{w} / \mathrm{H}_{2} \mathrm{O}$ at $8{ }^{\circ} \mathrm{C}$. Cold water extract obtained by stirring residue w/ distilled $\mathrm{H}_{2} \mathrm{O}$ for $12 \mathrm{hr}$ after neutralization $\mathrm{w} / .01 \mathrm{M} \mathrm{NaOH}$. Hot water extract by collecting residue, adjusting to $\mathrm{pH} 8$ w/ $\mathrm{NaOH}$, stirring $1 \mathrm{hr}$ at $120^{\circ} \mathrm{C}$. Extracts ppt'd w/ 2-propanol, dialyzed, lyophilized. Further separated by solubility in .1M KCl. 1H NMR (500 MHz, also $400 \mathrm{MHz}$; used especially to look for 3,6-anhydro and sulfated resonances) (Knutsen et al., 1990)

-disrupted cells, centrifuged to remove cellular contents, washed w/ solvents, suspended and dialyzed cell walls, lyophilized. Electron microscopy, phenol- $\mathrm{H}_{2} \mathrm{SO}_{4}$, chem. and enzymatic degradation, charcoal chromatography, paper chromatography, IR (Nevo and Sharon, 1969)

-polysaccharide separated from centrifuged medium by ppt'ing w/ cetylpyridinium chloride. Centrifuged, dissolved in $\mathrm{LiCl}$; ppt'd w/ EtOH, repeated several times, dried in dessicator. Ion exch chromatography, paper chromatography, alditol acetates, ci-MS (Kieras et al., 1976)

-(purification of fucose-containing, sulfated polysacc done according to a reference; no further explanat.) Used GPC to separate MW fractions, TLC, colorimetric tests for acetyl, sulfate, uronic acid content determinations. Alditol acetates, GC, NMR (400MHz; used only to see signals for anomeric, methyl (deoxy), acetyl protons), methanolysis, per-Omethylation, GC-ms, desulfation. (Nishino et al., 1991)

$-\mathrm{NaH}^{14} \mathrm{CO}_{3}$ used to grow cultures of Phaeocystis. Medium centrifuged, dialyzed, hydrolyzed w/ $1 \mathrm{~N} \mathrm{H}_{2} \mathrm{SO}_{4}, \mathrm{BaCO}_{3} \mathrm{ppt}$, evaporated, paper chromatography w/ standards. Gel filtrat w/ standards to determine MW, anthrone and radioactivity detection. (Guillard and Hellebust, 1971)

-a review (1990) of spectroscopic/colorimetric tests for uronic, sulfated saccharides; includes separation techniques for major components of red, brown, and green algae (ie, carageenans, etc.) (Percival and McDowell, 1990)

-phenol- $\mathrm{H}_{2} \mathrm{SO}_{4}$ used to determine concentration. Carbohydrate extracted repeatedly w/ boiling $\mathrm{H}_{2} \mathrm{O}$. Ppt w/ EtOH (1 vol) and acetone (3 vol.). Monosaccharide composition determined by acid hydrolysis (6N HCl@100ㄷ, 1 hr), GC. (Handa, 1969)

-phenol- $\mathrm{H}_{2} \mathrm{SO}_{4}$ to estimate carbohydrate concentration in filtered medium; cells analyzed for carbohydrate by hydrolysis w/ $80 \% \mathrm{H}_{2} \mathrm{SO}_{4}$ and phenol- $\mathrm{H}_{2} \mathrm{SO}_{4}$. Medium carbohydrate 
further analyzed by dialysis of filtered, rotovaped medium, lyophilization, hydrolysis $\mathrm{w} / 2$ $\mathrm{N}$ TFA for $6 \mathrm{hr}$ at $100^{\circ} \mathrm{C}$, Na-borohydride reduction, acetylation, GC. (Vieira and Myklestad, 1986)

-mucilage hydrolyzed in formic acid, paper chromatography, GLC, methylation, alditol acetates, GLC-MS (Fareed and Percival, 1977)

-coccoliths isolated by disruption, gradient centrifugation, EDTA, GPC, gel electrophoresis; coccoliths also treated w/ Na-phosphate buffer, $1 \mathrm{M} \mathrm{NaOH}, \mathrm{KMnO} 4$, equilib dialysis (to determine $\mathrm{Ca}++$ binding properties) (De Jong et al., 1976)

-(got polysacc from Marine Colloids Inc. Rockland, Maine). Hydrolyzed, alditol acetates, GC-MS, paper chrom., GPC, Hakomori methylation, periodate oxidation, desulfation, IR (Percival and Foyle, 1979)

-(only mentions polysacc fibrillar material in passing; doesn't even state clearly how it was removed to get at the sporopollenin-like material) (Berkaloff et al., 1983)

-total carbohydrate determination (technique not specified.) Surface-active carbohydrate removed by acidification of medium to $\mathrm{pH} \mathrm{5,} \mathrm{C18} \mathrm{sep-pak,} \mathrm{methanolysis,} \mathrm{TMS,} \mathrm{GC.}$

(Frew et al., 1990)

-medium filtered, dialyzed in 10-14kD membrane, lyophilized; gel chromatog, TMS derivs analyzed by GC, alditol acetates, GC-MS, desulfuration, IR (Paulsen et al., 1992)

-cells lyophilized, extracted w/ .05 $\mathrm{M} \mathrm{H}_{2} \mathrm{SO}_{4}$ for $1 \mathrm{hr}$ at $4{ }^{\circ} \mathrm{C}$. Neutralized w/ NaOH, dialyzed against $\mathrm{H}_{2} \mathrm{O}$, concentrated, lyophilized.GLC, GC-MS, methanolysis, periodate oxidation, etc. (Paulsen and Myklestad, 1978)

-cells washed, lyophilized, extracted w/ chloroform-MeOH- $\mathrm{H}_{2} \mathrm{O}$ to get 'low MW carbohydrate', then hydrolyzed w/ $.5 \mathrm{M} \mathrm{H}_{2} \mathrm{SO}_{4}$. Total carbohydrate by phenol- $\mathrm{H}_{2} \mathrm{SO}_{4}$; alditol acetates, GC. Total carbohydrate yield surprisingly low (4.6-12\% of organic matter) (Brown, 1991) 


\section{References}

Allan, G. G., J. Lewin and P. G. Johnson (1972). Marine Polymers. IV Diatom polysaccharides. Bot. Mar. 15: 102-108.

Berkaloff, C., E. Casadevall, C. Largeau, P. Metzger, S. Peracca and J. Virlet (1983). The resistant polymer of the walls of the hydrocarbon-rich alga Botryococcus braunii. Phytochem. 22: 389-397.

Brown, M. R. (1991). The amino-acid and sugar composition of 16 species of microalgae used in mariculture. J. Exp. Mar. Biol. Ecol. 145: 79-99.

Burney, C. M., P. G. Davis, K. M. Johnson and J. M. Sieburth (1981). Dependence of dissolved carbohydrate concentrations upon small scale nanoplankton and bacterioplankton distributions in the Western Sargasso Sea. Mar. Biol. 65: 289-296.

De Jong, E. W., L. Bosch and P. Westbroek (1976). Isolation and characterization of a $\mathrm{Ca}^{2+}$-binding polysaccharide associated with coccoliths of Emiliania huxleyi (Lohmann) Kamptner. Eur. J. Biochem. 70: 611-621.

Fareed, V. S. and E. Percival (1977). The presence of rhamnose and 3-O-methylxylose in the extracellular mucilage from the red alga Rhodella maculata. Carbohydr. Res. 53: 276277.

Fichtinger-Schepman, A. M., J. P. Kamerling, J. F. G. Vliegenthart, E. W. De Jong, L. Bosch and P. Westbroek (1979). Composition of a methylated, acidic polysaccharide associated with coccoliths of Emiliania huxleyi (Lohmann) Kamptner. Carbohydr. Res. 69: 181-189.

Ford, C. W. and E. Percival (1965). Carbohydrates of Phaeodactylum tricornutum. Part II. A sulphated glucuronomannan. J. Chem. Soc. : 7042-7046.

Frew, N. M., J. C. Goldman, M. R. Dennett and A. S. Johnson (1990). Impact of phytoplankton-generated surfactants on air-sea gas exchange. J. Geophys. Res. 95(C3): 3337-3352.

Geresh, S., O. Dubinsky, S. Arad, D. Christiaen and R. Glaser (1990). Structure of the 30 -( $\alpha$-D-glucopyranosyluronic acid)-L-galactopyranose, an aldobiouronic acid isolated from the polysaccharides of various unicellular red algae. Carbohydr. Res. 208: 301-305.

Guillard, R. R. and P. J. Wangersky (1958). The production of extracellular carbohydrates by some marine flagellates. Limno. Oceano. 3(4): 449-454.

Guillard, R. R. L. and J. A. Hellebust (1971). Growth and the production of extracellular substances by two strains of Phaeocystis poucheti. J. Phycol. 7: 330-338.

Handa, N. (1969). Carbohydrate metabolism in the marine diatom Skeletonema costatum. Mar. Biol. 4: 208-214.

Haug, A. and S. Myklestad (1976). Polysaccharides of marine diatoms with special reference to Chaetoceros species. Mar. Biol. 34: 217-222. 
Haug, A., S. Myklestad and E. Sakshaug (1973). Studies on the phytoplankton ecology of the Trondjeimsfjord. I. The chemical composition of phytoplankton populations. J. exp. mar. Biol. Ecol. 11: 15-26.

Hecky, R. E., K. Mopper, P. Kilham and E. T. Degens (1973). The amino acid and sugar composition of diatom cell-walls. Mar. Biol. 19: 323-331.

Jaseja, M., A. S. Perlin, O. Dubinsky, D. Christiaen, S. Arad and R. Glaser (1989). N.m.r. structure determination of 3-0-( $\alpha$-D-glucopyranosyluronic acid)-Lgalactopyranose, an aldobiuronic acid isolated from the unicellular red alga Rhodella reticulata. Carbohydr. Res. 186: 313-319.

Kieras, J. H., F. J. Kieras and D. V. Bowen (1976). 2-O-methyl-D-glucuronic acid, a new hexuronic acid of biological origin. Biochem. J. 155: 181-185.

Knutsen, S. H., D. E. Myslabodski and H. Grasdalen (1990). Characterization of carrageenan fractions from Norwegian Furcellaria lumbricalis (Huds.) Lamour. by $1 \mathrm{H}-$ NMR spectroscopy. Carbohydr. Res. 206: 367-372.

Lancelot, C. (1979). Gross excretion rates of natural marine phytoplankton and heterotrophic uptake of excreted products in the southern North Sea, as determined by short-term kinetics. Mar. Ecol. Prog. Ser 1: 179-186.

Lee, R. E. (1980). Phycology. Cambridge, Cambridge University Press.

Lupescu, N., S. Arad, S. Geresh, M. A. Bernstein and R. Glaser (1991). Structure of some sulfated sugars isolated after acid hydrolysis of the extracellular polysaccharide of Porphyridium sp., a unicellular red alga. Carbo. Res. 210: 349-352.

Maeda, M., Fukushi-Fujikura and O. Otsuru (1990). Cellulose in the cell wall of the siphonous green alga, Bryopsis maxima. Carbohydr. Res. 207: 91-99.

Mykelstad, S. (1977). Production of carbohydrates by marine planktonic diatoms. II. Influence of the N/P ratio in the growth and medium on the assimilation ratio, growth rate, and production of cellular and extracellular carbohydrates by Chaetoceros affinis var Willei (Gran) Hustedt and Skeletonema costatum (Grev.) Cleve. J. exp. mar. Biol. Ecol. 29: 161-179.

Myklestad, S. (1974). Production of carbohydrates by marine planktonic diatoms. I. Comparison of nine different species in culture. J. exp. mar. Biol. Ecol. 15: 261-274.

Myklestad, S. and A. Haug (1972). Production of carbohydrates by the marine diatom Chaetoceros affinis var. Willei (Gran) Hustedt. I. Effect of the concentration of nutrients in the culture medium. J. exp mar. Biol. Ecol. 9: 125-136.

Myklestad, S., A. Haug and B. Larsen (1972). Production of carbohydrates by the marine diatom Chaetoceros Affinis var Willei (Gran) Hustedt. II. Preliminary investigation of the extracellular polysaccharide. J. exp. mar. Biol. Ecol. 9: 137-144.

Myklestad, S., O. Holm-Hansen, K. M. Varum and B. E. Volcani (1989). Rate of release of extracellular amino acids and carbohydrates from the marine diatom Chaetoceros affinis. J. Plankton Res. 11(4): 763-773. 
Nevo, Z. and N. Sharon (1969). The cell wall of Peridinium westii, a non cellulosic glucan. Biochem. Biophys. Acta 173: 161-175.

Nishino, T., T. Nagumo, H. Kiyohara and H. Yamada (1991). Structural characterization of a new anticoagulant fucan sulfate from the brown seaweed Ecklonia kurome.

Carbohydr. Res. 211: 77-90.

Painter, T. J. (1983). Algal Polysaccharides. The Polysaccharides. Academic Press. 195285.

Parsons, T. R., K. Stephens and J. D. H. Strickland (1961). On the chemical composition of eleven species of marine phytoplankton. J. Fish. Res. Bd. Canada 18(6): 1001-1016.

Paulsen, B. S., A. Haug and B. Larsen (1978). Structural studies of a carbohydratecontaining polymer present in the mucilage tubes of the diatom Berkeleya rutilans (Trent.) Grun. Carbohydr. Res. 66: 103-111.

Paulsen, B. S. and S. Myklestad (1978). Structural studies of the reserve glucan produced by the marine diatom Skeletonema costatum (grev.) Cleve. Carbohydr. Res. 62: 386-388.

Paulsen, B. S., A. A. H. Vieira and D. Klaveness (1992). Structure of extracellular polysaccharides produced by a soil Cryptomonas sp. (Cryptophyceae). J. Phycol. 28: 6163.

Percival, E. and R. A. Foyle (1979). The extracellular polysaccharide of Porphyridium cruentum and Porphyridium aerugineum. Carbohydr. Res. 72: 165-176.

Percival, E. and R. H. McDowell (1990). Algal polysaccharides. Methods in Plant Biochemistry. Academic Press. 523-547.

Percival, E., M. A. Rahman and H. Weigel (1980). Chemistry of the polysaccharides of the diatom Coscinodiscus nobilis. Phytochem. 19: 809-811.

Smestad, B., A. Haug and A. Myklestad (1974). Production of carbohydrates by the Marine Diatom Chaetoceros affinis var. Willei (Gran) Hustedt. III. Structural studies of the extracellular polysaccharide. Acta Chem. Scand. 28: 662-666.

Smestad, B., A. Haug and S. Myklestad (1975). Structural studies of the extracellular polysaccharide produced by the diatom Chaetoceros curvisetus Cleve. Acta Chem. Scand. B 29: 337-340.

Stevenson, T. T. and R. H. Furneaux (1991). Chemical methods for the analysis of sulphated galactans from red algae. Carbohydr. Chem. 210: 227-298.

Varum, K. M., J. K. Bjarne and S. Myklestad (1986). Structure of a food-reserve $\beta$-Dglucan produced by the haptophyte alga Emiliania huxleyi (Lohmann) Hay and Mohler. Carbohydr. Res. 152: 243-248.

Vieira, A. A. H. and S. Myklestad (1986). Production of extracellular carbohydrate in cultures of Ankistrodesmus densus Kors. (Chlorophyceae). J. Plankton Res. 8(5): 985994. 
Zelibor, J. L., jr., L. Romankew, P. G. Hatcher and R. R. Colwell (1988). Comparative analysis of the chemical composition of mixed and pure cultures of green algae and their decomposed residues by $13 \mathrm{C}$ nuclear magnetic resonance spectroscopy. Appl. Environ. Microbiol. 54(4): 1051-1060. 